\title{
Extended phase-space analysis of the Hořava-Lifshitz cosmology
}

\author{
Genly Leon $^{1, \mathrm{a}} \mathbb{D}$, Andronikos Paliathanasis ${ }^{2, \mathrm{~b}}$ \\ ${ }^{1}$ Departamento de Matemáticas, Universidad Católica del Norte, Avda. Angamos 0610, Casilla, 1280 Antofagasta, Chile \\ 2 Institute of Systems Science, Durban University of Technology, PO Box 1334, Durban 4000, South Africa
}

Received: 9 March 2019 / Accepted: 20 August 2019 / Published online: 7 September 2019

(C) The Author(s) 2019

\begin{abstract}
We examine the phase space of Hořava-Lifshitz cosmology for a wide range of self-interacting potentials for the scalar field under the detailed-balance condition and without imposing it, by means of the powerful method of $f$ devisers. A compactification approach is performed for the exponential potential and for potentials beyond the exponential one, extending the previous findings in the literature. By using this approach it is possible to describe the finite region of the phase space and the region where the phase-space variables becomes infinity. Furthermore, we present several results concerning the stability of the de Sitter solution in Hořava-Lifshitz cosmology using Center Manifold theory. The advantages of this procedure are unveiled immediately when it is compared with the Normal Forms Calculations presented before in the literature.
\end{abstract}

\section{Introduction}

Hořava-Lifshitz (HL) gravity is a power-counting renormalizable theory with consistent ultra-violet (UV) behavior exhibiting an anisotropic, Lifshitz scaling between time and space at the UV limit and contains General Relativity (GR) as an infrared (IR) fixed point [1]. HL theory is closely related to the Einstein-æther theory [2-10], which is an effective field theory, preserving locality and covariance, consisting of GR coupled at second derivative order to a dynamical time-like unit vector field, the æther. In the IR limit of extended Hořava gravity [1,2], the aether vector is assumed to be hypersurfaceorthogonal. That implies that every hypersurface-orthogonal Einstein-æther solution is a Hořava solution [11]. However while the latter equivalence is true in terms of exact solution it is not a general true for other results which follow from the direct form of the field equations, like the PPN constraints and other [12]. In the recent years there has been a lot of inter-

\footnotetext{
a e-mail: genly.leon@ucn.cl

be-mail: anpaliat@phys.uoa.gr
}

est in some aspects of the HL theory, and several analyzes have been carried on, e.g., in [13-33]. To reduce the number of terms in the Lagrangian formulation one may impose the detailed-balance condition [1]. Under detailed -balance, the $(D+1)$-dimensional theory acquires the renormalization properties of the $D$-dimensional one, that is, the theory has a quantum inheritance principle. However, there is a discussion whether the detailed -balance condition leads to reliable results or if it is able to reveal the full information of HořavaLifshitz gravity [34,35]. Therefore, one needs to investigate also the phenomenology when the detailed-balance condition is abandoned. One important application of HL gravity is that of the HL cosmology [34-64]. In [65] it was proposed a possible solution to the flatness problem, in which it is assumed that the initial condition of the Universe is set by a small instanton respecting the same scaling. In [66] it is analyzed the electromagnetic-gravity interaction in a pure HL framework formulated in $4+1$ dimensions and it is performed a Kaluza-Klein reduction to $3+1$ dimensions. In [67] it was studied a noncommutative version of the FriedmannRobertson-Walker (FRW) cosmological models within the gravitational HL theory. The matter content of the models is described by a perfect fluid and the constant curvature of the spatial sections may be positive, negative or zero. In [68] it is examined the Hamiltonian dynamics of bouncing Bianchi IX cosmologies with three scale factors in HL gravity. It is considered a positive cosmological constant plus non-interacting dust and radiation as the matter content of the models and the dynamics is presented in a six-dimensional phase space.

In the review [69] are discussed some recent developments of the HL theory: (i) universal horizons, black holes and their thermodynamics; (ii) non-relativistic gauge/gravity duality; and (iii) quantization of the theory. In the reference [70] was tested HL cosmological scenarios against the new observational constraints: an updated cosmological data set from Cosmic Microwave Background (Planck CMB), expansion rates of elliptical and lenticular galaxies, JLA compilation (Joint Light-Curve Analysis) data for Type Ia supernovae 
(SneIa), Baryon Acoustic Oscillations (BAO) and priors on the Hubble parameter with an alternative parametrization of the equations. The authors considered the curvature parameter as a free parameter in the analysis, and they found that the detailed-balance scenario exhibits positive spatial curvature to more than $3 \sigma$, whereas for further theory generalizations it was found evidence for positive spatial curvature at $1 \sigma$. Moreover, HL gravity is consistent with the recent observation of the gravitational-wave event GW170817 [71].

The thermodynamics of cosmological models in the HL theory of gravity for a perfect fluid was studied in detail in [72]. The cosmological evolution was studied, including the periods of deceleration and/or acceleration, and the existence of big bang, big crunch, and big rip singularities, and bouncing universe [72].

Though HL gravity received a lot of attention a decade ago, on its first stages of the theory, the difficulty to overpass some theoretical problems have lead the scientists to study other Lorentz violated theories such as the Einsteinæther theory, but still there are various groups which try to solve some of the problems of HL theory. For instance the problem to restore the Lorentz invariance on the lowenergy limit has been the subject of various studies. The most simple scenarios are that Lorentz invariance is recovered because the renormalization group flow of the system leads to emergent infrared Lorentz invariance, that supersymmetry protects infrared Lorentz invariance or a classically Lorentz invariant matter sector with controlled quantum corrections may simply coexist with HL gravity under certain conditions [73-76].

While there has been various attempts in the study of the renormalizability HL theory, its complete renormalizability has not yet been proven [77-80]. However, only recently, in [79] the renormalizability of the projectable HL gravity was proven. Projectable HL gravity does not provide the limit of GR, however it has many common physical properties with GR and that makes it an interesting case of study. Other open subjects of HL gravity are the renormalization group flow [81], the coupling with matter and the effects of Lorentz violation $[82,83]$.

Even though the theory has some drawbacks enumerated in Sect. 2, still there are found several attempts to cure some of these problems. In [32] the authors reconsidered the "detailed balance" as a principle that can be used to restrict the proliferation of couplings in HL gravity, and for the usual shortcomings -that have been usually associated with it in the literature- were found easy resolutions for all of them within the framework of detailed balance, but the most persistent is related to the projectability. The fact some of these issues are very difficult to be answered, however, does not spoil the interest in the theory. Recently, it was increased the interest in finding solutions in HL gravity and in Einsteinæther theory. Recently in [84] it was presented a procedure to construct anisotropic interior solutions in Hořava gravity and Einstein- æther theory and in [85] it was investigated interior solutions with anisotropic fluids in the context HL gravity and Einstein- æther theory with non-static æther field, with the field equations becoming solvable. In [86] we have found exactly solvable models for a prototype of HL cosmology imposing the so called projectability condition, and considering the "detailed balance" condition [41]. In particular, we performed a detailed study of the integrability of the HL scalar field cosmology in a FLRW background spacetime. More specifically, we have tested if the gravitational field equations possesses the Painlevé property. For the exponential potential of the scalar field we are able to perform an analytic explicit integration of the field equations and write the solution in terms of a Right Painlevé series. Relativistic polytropic equations of state in the context of HL gravity and Einstein-æther theory were examined in [87].

In this paper we will be focused more about the general evolution of the solution space for the model. By this it is understood: (i) to obtain a global visual picture of the solutions space, by characterizing, locally and globally, the so-called attractor solution; (ii) to prove issues concerning asymptotic past and future behavior, (iii) generalizing and simplifying earlier proofs in the literature, and (iv) using the Center Manifold Theory to rigorously analyze the stability of several cosmological solutions that are of interest for inflation in HL gravity.

In order to investigate the generic features of a general cosmological scenario one can apply the powerful method of dynamical system analysis, which allows to bypass the complexity of the involved equations and extract information about the global features and behavior of the cosmological scenario [88-92]. In the case of HL cosmology an initial investigation in this direction was performed in [41] for the case of exponential potential, in which the authors have found stable dark-energy-dominated solutions, as well as bouncing solutions. In [93] it was investigated the properties of cosmological models based on the HL gravity by using the dynamical system approach but using different normalization procedures in comparison with [41]. Lyapunov method has been used in some gravitational scenarios, say [94-98]. In the present work we provide a complete dynamical system analysis of HL cosmology keeping the potentials arbitrary, which is a major improvement since it allows for the extraction of information that is related to the foundations of the cosmological model and not to the specific potential form. In particular, we apply the method of $f$-devisers [99-103], in which one first performs the analysis without the need of an a priori specification of the potential, and in the end one just substitutes the specific potential form in the results, instead of having to repeat the whole dynamical elaboration from the start. As we will see, the results will be richer and more general, revealing the full capabilities of HL cosmology. 
The plan of the work is as follows. In Sect. 2 we briefly review the gravitational field equations in HL cosmology where for the underlying geometry we consider that of a isotropic and homogeneous universe. In Sect. 3 it is studied the spatially flat FRW universe $(k=0)$ without a cosmological constant term, i.e. $\Lambda=0$, under the detailedbalance condition. In Sect. 3.1 the phase space analysis for arbitrary potentials for the latter cosmological model is presented. Moreover, the case of the Exponential potential $V(\phi)=V_{0} e^{-s \phi}$, with $s$ constant is detailed studied in Sect. 3.2. In Sect. 4.3 it is studied a Powerlaw potential $V(\phi)=\frac{1}{2 n}(\mu \phi)^{2 n}, \mu>0, n=1,2, \ldots$ discussed previously in [104] for a conventional scalar field while in Sect. 3.4 it is studied the E-model with potential $V(\phi)=$ $V_{0}\left(1-e^{-\sqrt{\frac{2}{3 \alpha}} \phi}\right)^{2 n}$ discussed in [105] for a conventional scalar field cosmology. The methodology we use is different from that employed in [104,105], and we apply the potentials in a different context. However, we complimentary use analogous variables as in $[104,105]$ to make comparisons with the relativistic case. The analogous analysis is done in Sect. 4 for the universe nonzero spatial curvature and without cosmological constant term under the detailed-balance condition. Furthermore, in Sects. 5 and 6 we consider that $\Lambda \neq 0$ and we perform similar analysis for $k=0$ and $k \neq 0$ respectively. In Sect. 7 is is studied the HL model beyond the detailed-balance condition. With present several results concerning the stability of the de Sitter solution in HorravaLifshitz cosmology using the Center Manifold theory, summarized in Appendix A. The main results of this research are proved in Sects. 3.3.1, 3.3.3, 3.4.1 , 3.4.3, 4.3.1, 4.3.3, 4.4.1, 4.4.3, 5.3.2, 5.4.2, and in Sect. 7.2.1. We find that in all the cases, with the exception of the model studied in Sect. 7.2.1, the de Sitter solution is unstable: saddle or centersaddle. In Sect. 7.2.1 we prove that the de Sitter solution is locally asymptotically stable. The advantages of this procedure are unveiled immediately when it is compared with the Normal Forms Calculations presented before in the literature. Finally, Sect. 8 summarizes our results where also we draw our conclusions.

\section{The cosmological equations}

In the simple version of HL gravity [34,35] the line-element can be written as:

$d s^{2}=-N^{2} d t^{2}+g_{i j}\left(d x^{i}+N^{i} d t\right)\left(d x^{j}+N^{j} d t\right)$,

where the lapse and shift functions are respectively $N$ and $N_{i}$. The spatial metric is given by $g_{i j}$, and roman letters indicate spatial indices. The scaling transformation is $t \rightarrow l^{3} t$ and $x^{i} \rightarrow l x^{i}$.
Under the detailed-balance condition the full action of Hořava-Lifshitz gravity is given by

$$
\begin{aligned}
S_{g}= & \int d t d^{3} x \sqrt{g} N\left\{\frac{2}{\kappa^{2}}\left(K_{i j} K^{i j}-\lambda K^{2}\right)\right. \\
& +\frac{\kappa^{2}}{2 w^{4}} C_{i j} C^{i j}-\frac{\kappa^{2} \mu}{2 w^{2}} \frac{\epsilon^{i j k}}{\sqrt{g}} R_{i l} \nabla_{j} R_{k}^{l}+\frac{\kappa^{2} \mu^{2}}{8} R_{i j} R^{i j} \\
& \left.-\frac{\kappa^{2} \mu^{2}}{8(3 \lambda-1)}\left[\frac{1-4 \lambda}{4} R^{2}+\Lambda R-3 \Lambda^{2}\right]\right\}
\end{aligned}
$$

where $K_{i j}=\left(\dot{g}_{i j}-\nabla_{i} N_{j}-\nabla_{j} N_{i}\right) / 2 N$ is the extrinsic curvature and $C^{i j}=\epsilon^{i j k} \nabla_{k}\left(R_{i}^{j}-R \delta_{i}^{j} / 4\right) / \sqrt{g}$ the Cotton tensor, and the covariant derivatives are defined with respect to the spatial metric $g_{i j} . \epsilon^{i j k}$ is the totally antisymmetric unit tensor, $\lambda$ is a dimensionless constant and the quantities $\kappa, w$ and $\mu$ are constants. Furthermore, in order to avoid phantomlike solutions we assume $\gamma>\frac{1}{3}$, while for simplicity we select to work with units where $\kappa^{2}=8 \pi G=1$.

The matter source we consider the following action term $[34,35]$

$S=\int d t d^{3} x \sqrt{g} N\left[\frac{3 \lambda-1}{4} \frac{\dot{\phi}^{2}}{N^{2}}-V(\phi)\right]$,

where by simplicity we have not considered the higher derivative terms $\propto \phi \nabla^{2} \phi, \propto \phi \nabla^{4} \phi, \propto \phi \nabla^{6} \phi, \ldots$.

Cosmological Principle, of the homogeneous and isotropic universe, leads to the consideration of the FRW metric

$g_{i j}=a^{2}(t) \gamma_{i j}, N^{i}=0, \gamma_{i j} d x^{i} d x^{j}=\frac{d r^{2}}{1-k r^{2}}+r^{2} d \Omega_{2}^{2}$,

where $k=-1,0,+1$ corresponding to open, flat, and closed universe respectively. Without loss of generality in the following we select $N=1$.

Hence, for that specific line element, the equations are reduced to:

$$
\begin{aligned}
& H^{2}= \frac{1}{6(3 \lambda-1)}\left[\frac{3 \lambda-1}{4} \dot{\phi}^{2}+V(\phi)\right] \\
&+\frac{1}{16(3 \lambda-1)^{2}}\left[-\frac{\mu^{2} k^{2}}{a^{4}}-\mu^{2} \Lambda^{2}+\frac{2 \mu^{2} \Lambda k}{a^{2}}\right], \\
& \dot{H}+ \frac{3}{2} H^{2}=-\frac{1}{4(3 \lambda-1)}\left[\frac{3 \lambda-1}{4} \dot{\phi}^{2}-V(\phi)\right] \\
&-\frac{1}{32(3 \lambda-1)^{2}}\left[-\frac{\mu^{2} k^{2}}{a^{4}}+3 \mu^{2} \Lambda^{2}-\frac{2 \mu^{2} \Lambda k}{a^{2}}\right], \\
& \ddot{\phi}+3 H \dot{\phi}+\frac{2 V^{\prime}(\phi)}{3 \lambda-1}=0 .
\end{aligned}
$$

If the detailed -balance condition is removed, the gravitational action can be schematically written as $S_{g}=$ $\int d t d^{3} x \sqrt{g} N\left(\mathscr{L}_{K}+\mathscr{L}_{V}\right)$, where the gravitational action 
have been expanded in its kinetic and potential part. By varying $N$ and $g_{i j}$, we extract the Friedmann equations $[15,41]$ :

$$
\begin{gathered}
H^{2}=\frac{2}{(3 \lambda-1)}\left[\sigma_{0}\left(\frac{3 \lambda-1}{4} \dot{\phi}^{2}+V(\phi)\right)\right. \\
\left.+\frac{\sigma_{1}}{6}+\frac{\sigma_{2} k}{6 a^{2}}+\frac{\sigma_{3} k^{2}}{6 a^{4}}+\frac{\sigma_{4} k}{6 a^{6}}\right], \\
\dot{H}+\frac{3}{2} H^{2}=-\frac{3}{(3 \lambda-1)}\left[\sigma_{0}\left(\frac{3 \lambda-1}{4} \dot{\phi}^{2}-V(\phi)\right)\right. \\
\left.-\frac{\sigma_{1}}{6}-\frac{\sigma_{2} k}{6 a^{2}}+\frac{\sigma_{3} k^{2}}{18 a^{4}}+\frac{\sigma_{4} k}{6 a^{6}}\right], \\
\ddot{\phi}+3 H \dot{\phi}+\frac{2 V^{\prime}(\phi)}{3 \lambda-1}=0 .
\end{gathered}
$$

The constants $\sigma_{i}$ satisfy $\sigma_{0} \equiv \kappa^{2} / 12, \sigma_{2}<0, \sigma_{4}>0$.

To study the system (5), (6), (7), corresponding to HL cosmology under the detailed-balance condition, it is more convenient to introduce the auxiliary variables [41]:

$$
\begin{aligned}
& x=\frac{\dot{\phi}}{2 \sqrt{6} H}, \quad y=\frac{\sqrt{V(\phi)}}{\sqrt{6} H \sqrt{3 \lambda-1}}, \\
& z=\frac{\mu}{4(3 \lambda-1) a^{2} H}, \quad u=\frac{\Lambda \mu}{4(3 \lambda-1) H}, \\
& s=-\frac{V^{\prime}(\phi)}{V(\phi)}, \quad f \equiv \frac{V^{\prime \prime}(\phi)}{V(\phi)}-\frac{V^{\prime}(\phi)^{2}}{V(\phi)^{2}},
\end{aligned}
$$

together with $N=\ln a$ and assuming that $f$ can be written as an explicit function of $s$ we obtain a closed dynamical system. For the exponential potential $s$ is constant and $f \equiv 0$. Thus, the system's dimensionality is reduced in one dimension. Therefore, we extent the phase-space analysis of [41] by considering potentials beyond the exponential with non-trivial $f(s)$. Given $f(s)$, the stability of the hyperbolic equilibrium points is given by analyzing the signs of the reals parts of the eigenvalues of the matrix of linear perturbations $\mathbf{Q}$ evaluated at each equilibrium point. For studying the more general system (8), (9), (10), we can introduce the auxiliary variables [41]:

$$
\begin{aligned}
& x=\frac{\dot{\phi}}{2 \sqrt{6} H}, \quad y=\frac{\sqrt{V(\phi)}}{\sqrt{6} H \sqrt{3 \lambda-1}}, \\
& x_{1}=\frac{\sigma_{1}}{3(3 \lambda-1) H^{2}}, \quad x_{2}=\frac{k \sigma_{2}}{3(3 \lambda-1) a^{2} H^{2}}, \\
& x_{3}=\frac{\sigma_{3}}{3(3 \lambda-1) a^{4} H^{2}}, \quad x_{4}=\frac{2 k \sigma_{4}}{(3 \lambda-1) a^{6} H^{2}} .
\end{aligned}
$$

For the common scalar field potentials the function $f(s)$ is found as follows. The monomial potential $V(\phi)=$ $\frac{1}{2 n}(\mu \phi)^{2 n}, \mu>0, n=1,2, \ldots[104]$ has $f(s)=-\frac{s^{2}}{2 n}$. The so-called E-model studied from the dynamical systems point of view in [105] has potential $V(\phi)=V_{0}\left(1-e^{-\sqrt{\frac{2}{3 \alpha}} \phi}\right)^{2 n}$.
The corresponding $f$-deviser is $f(s)=-\frac{s(s-\sqrt{6} \mu)}{2 n}$, where $\mu=\frac{2 n}{3 \sqrt{\alpha}}$. The exponential potential plus a cosmological constant $V(\phi)=V_{0} e^{-l \phi}+V_{1}[106-108]$ has $f(s)=-s(s-$ l). The hyperbolic potentials: $V(\phi)=V_{0}(\cosh (\xi \phi)-1)$ [108-118] with $f(s)=-\frac{1}{2}\left(s^{2}-\xi^{2}\right)$ and $V(\phi)=$ $V_{0} \sinh ^{-\alpha}(\beta \phi)[108-113,119]$ with $f(s)=\frac{s^{2}}{\alpha}-\alpha \beta^{2}$. The double exponential potential $V(\varphi)=V_{0}\left(e^{\alpha \phi^{\alpha}}+e^{\beta \phi}\right)[120$ 122] has $f(s)=-(s+\alpha)(s+\beta)$.

The above basic models of HL cosmology proves to have very interesting cosmological behavior [34,35,40-62]. However, the gravitational sector itself proves to have instabilities that cannot be cured by simple tricks such as analytic continuation [15-18].

There are several physical drawbacks in the theory, say, the projectable HL does not provide the limit of GR, and when the projectability condition it is applied-where the lapse function is a global quantity with no variation over a constant time hypersurface- the theory has not a local Hamiltonian constraint [123]. Additionally, in the projectable theory, there are instability problems and strong coupling for cosmological solutions $[15,37,124]$. On the other hand, the theory with detailed balance in $3+1$ dimensions also it suffers from some problems (see [32], and references therein): there is a parity violating term which is of fifth order in derivatives [125,126]; the squared Cotton tensor, which appears in the theory, is traceless and vanishes for conformally flat three-dimensional spaces. Therefore, it does not contribute to the propagator of the scalar graviton. Hence, the scalar mode does not satisfy the usual dispersion relation and is not power-counting renormalizable, unlike the spin- 2 mode. This spoils the overall UV properties of the theory [1]. The infrared behavior of the scalar mode is plagued by instabilities and strong coupling at unacceptably low energies $[15,37]$. The (bare) cosmological constant has the opposite sign from the observed value $[125,126]$ and has to be large, much larger than the observed value [127].

Therefore, it is necessary to try to construct suitable extensions that are free of such problems. A quite general powercounting renormalizable action is [128]:

$S=S_{k i n}+S_{1}+S_{2}+S_{\text {new }}$,

with

$$
\begin{aligned}
S_{k i n}= & \alpha \int d t d^{3} x \sqrt{g} N\left[\left(K_{i j} K^{i j}-\lambda K^{2}\right)\right], \\
S_{1}= & \int d t d^{3} x \sqrt{g} N\left[\gamma_{0} \frac{\epsilon^{i j k}}{\sqrt{g}} R_{i l} \nabla_{j} R_{k}^{l}+\zeta R_{i j} R^{i j}\right. \\
& \left.+\eta R^{2}+\xi R+\sigma\right],
\end{aligned}
$$

$S_{2}=\int d t d^{3} x \sqrt{g} N\left[\beta_{0} C_{i j} C^{i j}+\beta_{1} R \square R+\beta_{2} R^{3}\right.$ 


$$
\left.+\beta_{3} R R_{i j} R^{i j}+\beta_{4} R_{i j} R^{i k} R_{k}^{j}\right]
$$

and

$$
\begin{aligned}
S_{\text {new }}= & \int d t d^{3} x \sqrt{g} N\left[a_{1}\left(a_{i} a^{i}\right)+a_{2}\left(a_{i} a^{i}\right)^{2}+a_{3} R^{i j} a_{i} a_{j}\right. \\
& \left.+a_{4} R \nabla_{i} a^{i}+a_{5} \nabla_{i} a_{j} \nabla^{i} a^{j}+a_{6} \nabla^{i} a_{i}\left(a_{j} a^{j}\right)+\cdots\right] .
\end{aligned}
$$

Thus, apart from the known kinetic, detailed-balance and beyond-detailed-balance combinations that constitute the HL gravitational action, in (17) it is added a new combination, based on the term $a_{i} \equiv \frac{\partial_{i} N}{N}$, which breaks the projectability condition, and the ellipsis in (17) refers to dimension six terms involving $a_{i}$ as well as curvatures [36,37]. Such a new combination of terms seems to alleviate the problems of HL gravity, although there could still be some ambiguities. Therefore, one should repeat all the relevant investigations of the literature for this extended version of the theory.

In this paper, however, we will be focused in the general evolution of the solution space for the model (5), (6), (7) (under the detailed-balance condition) for the situations: i): Flat universe with $\Lambda=0$. ii) Non-flat universe with $\Lambda=0$. iii) Case 3: Flat universe with $\Lambda \neq 0$. For completeness, we perform the dynamical systems analysis of the model: $k \neq$ $0, \Lambda \neq 0$ under the detailed-balance condition for arbitrary potentials at the finite region of the phase space. Furthermore, we study the solution space at the finite region of the phase space for the system (8), (9), (10) without detailed-balance.

\section{Case 1: Flat universe with $\Lambda=0$ under the detailed-balance condition}

The field equations in this example become:

$$
\begin{aligned}
& H^{2}=\frac{1}{6(3 \lambda-1)}\left[\frac{3 \lambda-1}{4} \dot{\phi}^{2}+V(\phi)\right], \\
& \dot{H}=-\frac{1}{8} \dot{\phi}^{2}, \\
& \ddot{\phi}+3 H \dot{\phi}+\frac{2 V^{\prime}(\phi)}{3 \lambda-1}=0 .
\end{aligned}
$$

Now, we discuss the phase space of this model for arbitrary potentials and next we specify for some potentials.

\subsection{Arbitrary potentials}

For an spatially flat spacetime without a cosmological constant whose evolution is given by (18), (19), (20), the corre- sponding autonomous system writes:

$$
\begin{aligned}
\frac{d x}{d N} & =(3 x-\sqrt{6} s)\left(x^{2}-1\right), \\
\frac{d z}{d N} & =\left(3 x^{2}-2\right) z, \\
\frac{d s}{d N} & =-2 \sqrt{6} x f(s) .
\end{aligned}
$$

defined on the phase space $\left\{(x, z, s) \in \mathbb{R}^{3}:-1 \leq x \leq 1\right\}$, where $N=\ln \left(a / a_{0}\right)$ denotes the new time variable.

The equilibrium points/curves of the dynamical system (21), (22), (23) at the finite region of the phase space are presented in Table 1, where is shown the existence and stability conditions. We proceed to the discussion of the more relevant features of them.

- $P_{1}(\hat{s}):(x, z, s)=(1,0, \hat{s})$. Where $\hat{s}$ denotes a value of $s$, such that $f(\hat{s})=0$. This point is reduced to $P_{1}$ studied in [41]. It is a source for $f^{\prime}(\hat{s})<0, \hat{s}<\sqrt{\frac{3}{2}}$.

$-P_{2}(\hat{s}):(x, z, s)=(-1,0, \hat{s})$. Where $\hat{s}$ denotes a value of $s$, such that $f(\hat{s})=0$. This point is reduced to $P_{2}$ studied in [41]. It is a source for $f^{\prime}(\hat{s})>0, \hat{s}>-\sqrt{\frac{3}{2}}$.

- $P_{3}(\hat{s}):(x, z, s)=\left(\sqrt{\frac{2}{3}} \hat{s}, 0, \hat{s}\right)$, where $\hat{s}$ denotes a value of $s$, such that $f(\hat{s})=0$ and $-\sqrt{\frac{3}{2}} \leq \hat{s} \leq \sqrt{\frac{3}{2}}$. This point is reduced to $P_{3}$ studied in [41]. It is a sink for $f^{\prime}(\hat{s})<0,-1<\hat{s}<0$, or $f^{\prime}(\hat{s})>0,0<\hat{s}<1$.

$-P_{3}^{0}:(x, z, s)=(0,0,0)$. This point is new, and it was not found in [41]. It is a sink for $f(0)>0$.

$-P_{4}^{+}:(x, z, s)=\left(\sqrt{\frac{2}{3}}, z_{c}, 1\right)$, where $z_{c}$ denotes an arbitrary number. Exists for $f(1)=0$. It is nonhyperbolic.

$-P_{4}^{-}:(x, z, s)=\left(-\sqrt{\frac{2}{3}}, z_{c},-1\right)$, where $z_{c}$ denotes an arbitrary number. Exists for $f(-1)=0$. It is nonhyperbolic. The above lines are reduced to the line $P_{4}$ studied in [41] when the analysis is restricted to the exponential potential (i.e., $s$ constant, $f \equiv 0$ ).

Owing to the fact that the dynamical system (21), (22), (23) is non-compact, there could be features in the asymptotic regime which are non-trivial for the global dynamics. Introducing the new variables

$Z=\frac{z}{\sqrt{1+z^{2}}}, \quad S=\frac{2}{\pi} \arctan (s)$.

The system (21), (22), (23) therefore becomes

$$
\begin{aligned}
& \frac{d x}{d N}=\left(x^{2}-1\right)\left(3 x-\sqrt{6} \tan \left(\frac{\pi S}{2}\right)\right), \\
& \frac{d Z}{d N}=\left(2-3 x^{2}\right) Z\left(Z^{2}-1\right),
\end{aligned}
$$


Table 1 Case 1: Equilibrium points at the finite region of the system (21), (22), (23)

\begin{tabular}{|c|c|c|c|c|}
\hline Equil. Points & $(x, z, s)$ & Existence & Eigenvalues & Stability \\
\hline$P_{1}(\hat{s})$ & $(1,0, \hat{s})$ & $f(\hat{s})=0$ & $6-2 \sqrt{6} \hat{s}, 1,-2 \sqrt{6} f^{\prime}(\hat{s})$ & $\begin{array}{l}\text { Nonhyperbolic for } f^{\prime}(\hat{s})=0 \text {, or } \hat{s}=\sqrt{\frac{3}{2}} \text {. } \\
\text { Source for } f^{\prime}(\hat{s})<0, \hat{s}<\sqrt{\frac{3}{2}} \\
\text { Saddle otherwise }\end{array}$ \\
\hline$P_{2}(\hat{s})$ & $(-1,0, \hat{s})$ & $f(\hat{s})=0$ & $6+2 \sqrt{6} \hat{s}, 1,2 \sqrt{6} f^{\prime}(\hat{s})$ & $\begin{array}{l}\text { Nonhyperbolic for } f^{\prime}(\hat{s})=0 \text {, or } \hat{s}=-\sqrt{\frac{3}{2}} \\
\text { Source for } f^{\prime}(\hat{s})>0, \hat{s}>-\sqrt{\frac{3}{2}} \\
\text { Saddle otherwise }\end{array}$ \\
\hline$P_{3}(\hat{s})$ & $\left(\sqrt{\frac{2}{3}} \hat{s}, 0, \hat{s}\right.$ & $\begin{array}{l}f(\hat{s})=0 \\
-\sqrt{\frac{3}{2}} \leq \hat{s} \leq \sqrt{\frac{3}{2}}\end{array}$ & $2 \hat{s}^{2}-3,2\left(\hat{s}^{2}-1\right),-4 \hat{s} f^{\prime}(\hat{s})$ & $\begin{array}{l}\text { Nonhyperbolic for } f^{\prime}(\hat{s})=0 \text {, or } \\
\qquad \hat{s} \in\left\{-\sqrt{\frac{3}{2}},-1,0,1, \sqrt{\frac{3}{2}}\right\}\end{array}$ \\
\hline & & & & $\begin{array}{l}\text { Sink for } f^{\prime}(\hat{s})<0,-1<\hat{s}<0 \text {, or } \\
\qquad f^{\prime}(\hat{s})>0,0<\hat{s}<1 \\
\text { Saddle otherwise }\end{array}$ \\
\hline$P_{3}^{0}$ & $(0,0,0)$ & Always & $-2,-\frac{3}{2} \pm \frac{1}{2} \sqrt{9-48 f(0)}$ & $\begin{array}{l}\text { Nonhyperbolic for } f(0)=0 \\
\text { Sink for } f(0)>0 \\
\text { Saddle otherwise }\end{array}$ \\
\hline$P_{4}^{+}$ & $\left(\sqrt{\frac{2}{3}}, z_{c}, 1\right.$ & $z_{c} \in \mathbb{R}, f(1)=0$ & $-1,0,-4 f^{\prime}(1)$ & Nonhyperbolic \\
\hline$P_{4}^{-}$ & $\left(-\sqrt{\frac{2}{3}}, z_{c}\right.$, & $z_{c} \in \mathbb{R}, f(-1)=0$ & $-1,0,4 f^{\prime}(-1)$ & Nonhyperbolic \\
\hline
\end{tabular}

$\frac{d S}{d N}=-\frac{2 \sqrt{6} x(\cos (\pi S)+1) f\left(\tan \left(\frac{\pi S}{2}\right)\right)}{\pi}$,

defined on the compact phase space

$\left\{(x, Z, S) \in \mathbb{R}^{3}:-1 \leq x \leq 1,-1 \leq Z \leq 1,-1 \leq S \leq 1\right\}$.

The points at the finite region of the phase space and their stability remains the same (under the rescaling $s=\tan \left(\frac{\pi S}{2}\right)$ ). The points at the infinite region of the phase space are summarized in Table 2. Now we discuss the relevant features of them.

- $Q_{1,2}(\hat{s}):(x, Z, S)=\left(-1, \pm 1, \frac{2}{\pi} \arctan (\hat{s})\right)$, where $f(\hat{s})=0$. It is a sink for $f^{\prime}(\hat{s})<0, \hat{s}<-\sqrt{\frac{3}{2}}$.

$-Q_{3,4}(\hat{s}): \quad(x, Z, S)=\left(1, \pm 1, \frac{2}{\pi} \arctan (\hat{s})\right)$ where $f(\hat{s})=0$. It is a sink for $f^{\prime}(\hat{s})>0, \hat{s}>\sqrt{\frac{3}{2}}$.

- $Q_{5,6}(\hat{s}):(x, Z, S)=\left(\sqrt{\frac{2}{3}} \hat{s}, \pm 1, \frac{2}{\pi} \arctan (\hat{s})\right)$, where $f(\hat{s})=0,-\sqrt{\frac{3}{2}} \leq \hat{s} \leq \sqrt{\frac{3}{2}}$. It is a sink for $-\sqrt{\frac{3}{2}}<\hat{s}<$ $-1, f^{\prime}(\hat{s})<0$, or $1<\hat{s}<\sqrt{\frac{3}{2}}, f^{\prime}(\hat{s})>0$.

- $Q_{7,8}:(x, Z, S)=(0, \pm 1,0)$. These points are saddle points.
These points at infinity satisfies $z \rightarrow \operatorname{sgn}(\mu) \infty$, which means $a^{2} H \rightarrow 0$. This includes Minkowski and static solutions.

\subsection{Exponential potential}

As Example 1, we implement the aforementioned procedure for the exponential potential $V(\phi)=V_{0} e^{-s \phi}$ [41], where $s$ is constant and $f$ is identically zero.

In this case the system (21), (22) becomes

$\frac{d x}{d N}=\left(x^{2}-1\right)(3 x-\sqrt{6} s)$,
$\frac{d Z}{d N}=\left(2-3 x^{2}\right) Z\left(Z^{2}-1\right)$,

defined on the compact phase space

$\left\{(x, z) \in \mathbb{R}^{2}:-1 \leq x \leq 1,-1 \leq Z \leq 1\right\}$.

The equilibrium points $P_{1,2}$ are not relevant from a cosmological point of view, since apart from being unstable they correspond to complete dark matter domination, with the matter equation-of-state parameter being stiff.

The equilibrium point $P_{3}$ can be the late-time state of the universe. If additionally we desire to keep the dark-matter equation-of-state parameter in the physical range $0<w_{M}<$ 1 then we have to restrict the parameter $s$ in the range $\frac{\sqrt{3}}{2}<$ 
Table 2 Case 1: Equilibrium points at the infinity region of the system (21), (22), (23)

\begin{tabular}{|c|c|c|c|c|}
\hline Equil. Points & $(x, Z, S)$ & Existence & Eigenvalues & Stability \\
\hline$Q_{1,2}(\hat{s})$ & $\left(-1, \pm 1, \frac{2}{\pi} \arctan (\hat{s})\right)$ & $f(\hat{s})=0$ & $-2,2 \sqrt{6} \hat{s}+6,2 \sqrt{6} f^{\prime}(\hat{s})$ & $\begin{array}{l}\text { Nonhyperbolic for } f^{\prime}(\hat{s})=0 \text {, or } \hat{s}=-\sqrt{\frac{3}{2}} \\
\text { Sink for } f^{\prime}(\hat{s})<0, \hat{s}<-\sqrt{\frac{3}{2}} \\
\text { It is a saddle otherwise }\end{array}$ \\
\hline$Q_{3,4}(\hat{s})$ & $\left(1, \pm 1, \frac{2}{\pi} \arctan (\hat{s})\right)$ & $f(\hat{s})=0$ & $-2,6-2 \sqrt{6} \hat{s},-2 \sqrt{6} f^{\prime}(\hat{s})$ & $\begin{array}{l}\text { Nonhyperbolic for } f^{\prime}(\hat{s})=0 \text {, or } \hat{s}=\sqrt{\frac{3}{2}} \\
\text { It is a sink for } f^{\prime}(\hat{s})>0, \hat{s}>\sqrt{\frac{3}{2}} \\
\text { It is a saddle otherwise }\end{array}$ \\
\hline$Q_{5,6}(\hat{s})$ & $\left(\sqrt{\frac{2}{3}} \hat{s}, \pm 1, \frac{2}{\pi} \arctan (\hat{s})\right)$ & $\begin{array}{l}f(\hat{s})=0 \\
-\sqrt{\frac{3}{2}} \leq \hat{s} \leq \sqrt{\frac{3}{2}}\end{array}$ & $4\left(1-\hat{s}^{2}\right), 2 \hat{s}^{2}-3,-4 \hat{s} f^{\prime}(\hat{s})$ & $\begin{array}{l}\text { Nonhyperbolic for } f^{\prime}(\hat{s})=0 \text {, or } \\
\qquad \hat{s} \in\left\{-\sqrt{\frac{3}{2}},-1,1, \sqrt{\frac{3}{2}}\right\} \\
\text { Sink for }-\sqrt{\frac{3}{2}}<\hat{s}<-1, f^{\prime}(\hat{s})<0 \text {, or } \\
\quad 1<\hat{s}<\sqrt{\frac{3}{2}}, f^{\prime}(\hat{s})>0 \text {. } \\
\text { Saddle otherwise }\end{array}$ \\
\hline$Q_{7,8}$ & $(0, \pm 1,0)$ & Always & $4,-\frac{1}{2}(3 \pm \sqrt{9-48 f(0)})$ & Saddle \\
\hline
\end{tabular}

$s<\sqrt{\frac{3}{2}}$. However, even in this case the universe is finally completely dominated by dark matter. The fact that $z_{c}=$ 0 means that in general this sub-class of universes will be expand forever. The equilibrium points $P_{4}$ consist a stable late-time solution, with a physical dark-matter equation-ofstate parameter $w_{M}=1 / 3$, but with zero dark energy density. We mention that the dark-matter domination of the case at hand was expected, since in the absent of curvature and of a cosmological constant the corresponding Hoř ava-Lifshitz universe is comprised only by dark matter. Note however that the dark-energy equation-of-state parameter can be arbitrary.

Figure 1a and c illustrates when $P_{3}$ becomes an stable line of equilibrium points for the specific values $s= \pm 1$. Figure 1 b illustrates when $P_{3}$ is an isolated late-time attractor. Figure 1d illustrates when $Q_{5,6}:(x, Z)=\left(\sqrt{\frac{2}{3}} s, \pm 1\right)$ is an attractor at the region at infinity for $-\sqrt{\frac{3}{2}}<s<-1<0$, or $1<s<\sqrt{\frac{3}{2}}$.

\subsection{Powerlaw potential}

As the Example 2, we consider the potential [104]:

$V(\phi)=\frac{1}{2 n}(\mu \phi)^{2 n}, \quad \mu>0, \quad n=1,2, \ldots$

such that

$f(s)=-\frac{s^{2}}{2 n}, \quad f(s)=0 \Leftrightarrow s=0, \quad f^{\prime}(0)=0$.
The system (25), (26), (27) becomes

$$
\begin{aligned}
& \frac{d x}{d N}=\left(x^{2}-1\right)\left(3 x-\sqrt{6} \tan \left(\frac{\pi S}{2}\right)\right), \\
& \frac{d Z}{d N}=\left(2-3 x^{2}\right) Z\left(Z^{2}-1\right), \\
& \frac{d S}{d N}=-\frac{\sqrt{6} x(\cos (\pi S)-1)}{\pi n},
\end{aligned}
$$

defined on the compact phase space

$$
\left\{(x, S, Z) \in \mathbb{R}^{3}:-1 \leq x \leq 1,-1 \leq S \leq 1,-1 \leq Z \leq 1\right\} .
$$

The equilibrium points of (32), (33), (34), and their stability conditions are summarized as follows.

- $P_{1}(0):(x, Z, S):=(1,0,0)$. Eigenvalues $\{6,1,0\}$. Unstable.

- $P_{2}(0):(x, Z, S):=(-1,0,0)$. Eigenvalues $\{6,-5,0\}$. Saddle.

$-P_{3}^{0}:(x, Z, S):=(0,0,0)$. Eigenvalues $\{-3,-2,0\}$. Nonhyperbolic.

- $Q_{1,2}(0):(x, Z, S):=(-1, \pm 1,0)$. Eigenvalues $\{10,6,0\}$. Unstable.

- $Q_{3,4}(0):(x, Z, S):=(1, \pm 1,0)$. Eigenvalues $\{6,-2,0\}$. Saddle.

- $Q_{5,6}(0):(x, Z, S):=(0, \pm 1,0)$. Eigenvalues $\{4,-3,0\}$. Saddle. 
Fig. 1 Compact phase portrait of (28), (29) for different choices of the parameter $s$
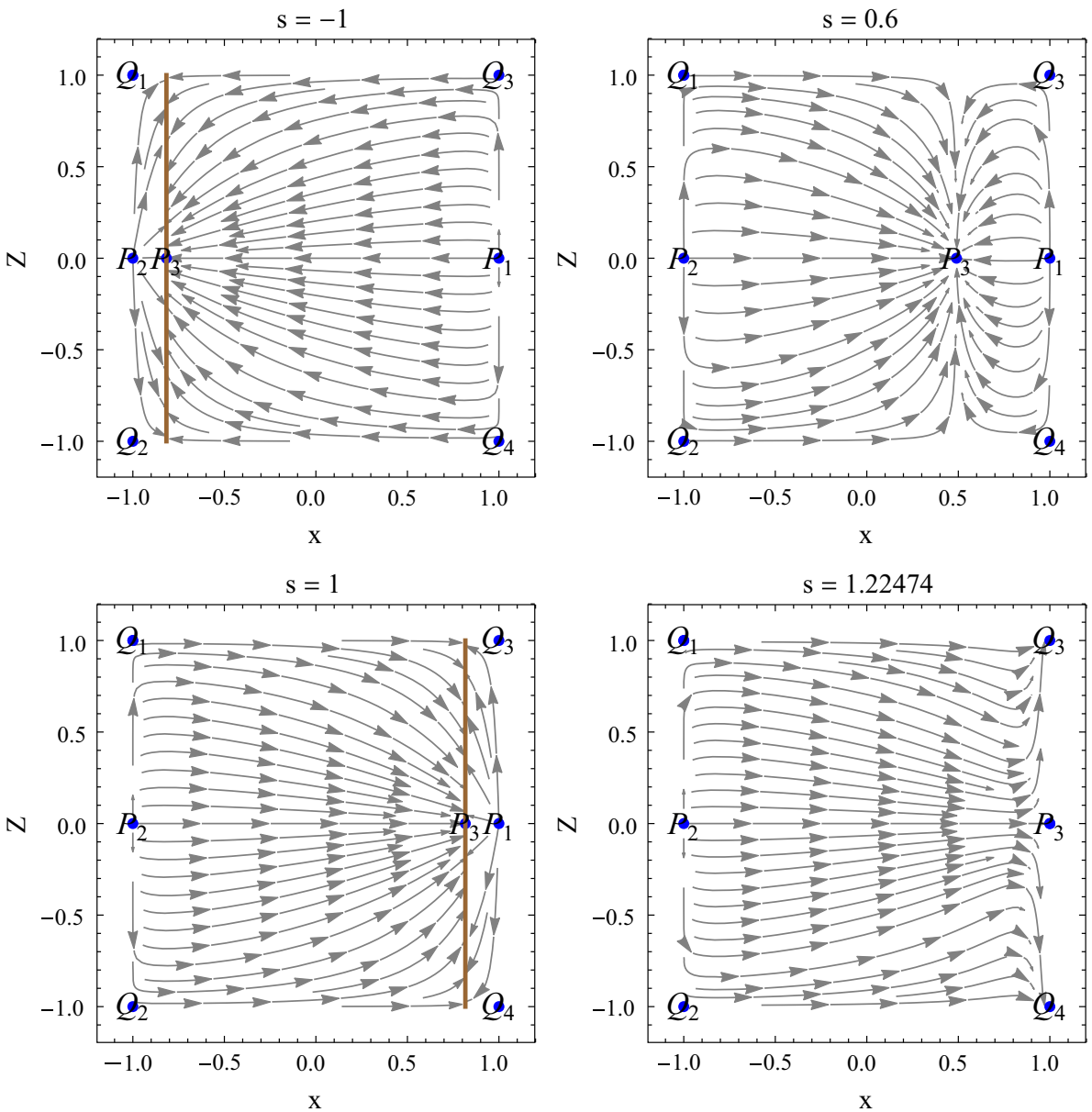

3.3.1 Stability analysis of the solution $P_{3}^{0}$ in

Hoŕava-Lifshitz cosmology for the Flat universe with $\Lambda=0$ under the detailed-balance condition for the powerlaw-potential

Proposition 1 The origin for the system (32), (33), (34) is unstable (saddle point).

Proof Taking the linear transformation

$\left(u, v_{1}, v_{2}\right)=\left(S, \frac{1}{6}(6 x-\sqrt{6} \pi S), Z\right)$,

and taking Taylor series near $\left(u, v_{1}, v_{2}\right)=(0,0,0)$ up to fifth order we obtain the system (32) can be written into its Jordan canonical form:

$$
\left(\begin{array}{l}
\frac{d u}{d N} \\
\frac{d v_{1}}{d N} \\
\frac{d v_{2}}{d N}
\end{array}\right)=\left(\begin{array}{ccc}
0 & 0 & 0 \\
0 & -3 & 0 \\
0 & 0 & -2
\end{array}\right)\left(\begin{array}{c}
u \\
v_{1} \\
v_{2}
\end{array}\right)+\left(\begin{array}{c}
f(u, \mathbf{v}) \\
g_{1}(u, \mathbf{v}) \\
g_{2}(u, \mathbf{v})
\end{array}\right),
$$

where

$$
\begin{aligned}
f(u, \mathbf{v}) & =-\frac{\pi u^{2}\left(\pi^{2} u^{2}-12\right)\left(\pi u+\sqrt{6} v_{1}\right)}{24 n}, \\
g_{1}(u, \mathbf{v}) & =\frac{\pi^{2} u^{2} v_{1}\left(\pi^{2}(1-2 n) u^{2}+12(n-1)\right)}{24 n}
\end{aligned}
$$

$$
\begin{aligned}
- & \frac{\pi u\left(\pi^{2} u^{2}-24\right) v_{1}^{2}}{4 \sqrt{6}}+3 v_{1}^{3} \\
& +\frac{\pi^{5}(5-2 n) u^{5}+30 \pi^{3}(n-2) u^{3}}{120 \sqrt{6} n}, \\
g_{2}(u, \mathbf{v})= & 2 v_{2}^{3}-\frac{1}{2} v_{2}\left(v_{2}^{2}-1\right) \\
& \left(\pi^{2} u^{2}+2 \sqrt{6} \pi u v_{1}+6 v_{1}^{2}\right) .
\end{aligned}
$$

According to Theorem 1, there exists a 1-dimensional invariant local center manifold $W^{c}(\mathbf{0})$ of $(36), W^{c}(\mathbf{0})=$ $\left\{(u, \mathbf{v}) \in \mathbb{R} \times \mathbb{R}^{2}: \mathbf{v}=\mathbf{h}(u)\right\}$, satisfying $\mathbf{h}(0)=\mathbf{0}$, $D \mathbf{h}(0)=\mathbf{0},|u|<\delta$ for $\delta$ sufficiently small. The restriction of (36) to the center manifold is $\frac{d u}{d N}=f(u, \mathbf{h}(u))$, where the function $\mathbf{h}(u)$ satisfies (A.10):

$D \mathbf{h}(u)[f(u, \mathbf{h}(u))]-P \mathbf{h}(u)-\mathbf{g}(u, \mathbf{h}(u))=0$,

where

$P=\left(\begin{array}{cc}-3 & 0 \\ 0 & -2\end{array}\right)$ 
According to Theorem 3, the system (37) can be solved approximately by expanding $\mathbf{h}(u)$ in Taylor series at $u=0$. Since $\mathbf{h}(0)=\mathbf{0}$ and $D \mathbf{h}(0)=\mathbf{0}$, we propose the ansatsz

$\mathbf{h}(u):=\left(\begin{array}{l}h_{1}(u) \\ h_{2}(u)\end{array}\right)=\left(\begin{array}{l}\sum_{j=1}^{4} a_{j} u^{j+1}+O\left(u^{6}\right) \\ \sum_{j=1}^{4} b_{j} u^{j+1}+O\left(u^{6}\right)\end{array}\right)$,

to substitute into (37). By comparing the coefficients of the equal powers of $u$ we find the non-null coefficients $a_{2}=$ $\frac{\pi^{3}(n-2)}{12 \sqrt{6} n}, a_{4}=\frac{\pi^{5}(n(3 n-25)+40)}{360 \sqrt{6} n^{2}}$.

Therefore, the center manifold can be represented locally by the graph

$v_{1}=\frac{(n-2) \pi^{3} u^{3}}{12 \sqrt{6} n}+\frac{(n(3 n-25)+40) \pi^{5} u^{5}}{360 \sqrt{6} n^{2}}, v_{2}=0$.

That is,

$x=\frac{\pi S}{\sqrt{6}}+\frac{\pi^{3}(n-2) S^{3}}{12 \sqrt{6} n}+\frac{\pi^{5}(n(3 n-25)+40) S^{5}}{360 \sqrt{6} n^{2}}, Z=0$.

The dynamics on the center manifold is given by the gradientlike equation

$\frac{d u}{d N}=-\nabla \Pi(u)$,

$\Pi(u)=-\frac{\pi^{2} u^{4}}{8 n}+\frac{\pi^{4} u^{6}}{72 n^{2}}-\frac{\left(\pi^{6}((n-40) n+80)\right) u^{8}}{11520 n^{3}}$.

We have $\Pi^{\prime}(0)=\Pi^{\prime \prime}(0)=\Pi^{\prime \prime \prime(4)}(0)=-\frac{3 \pi^{2}}{n}<0$. It follows that $u=0$ is a degenerated maximum of the potential. Using the Theorem 2, we conclude that the center manifold of origin for the system (36), and the origin itself are unstable (saddle point).

\subsubsection{Alternative compactification}

In this example we can alternatively introduce the following compactification inspired in the reference [104].

$$
\begin{aligned}
\Sigma & =\frac{\dot{\phi}}{2 \sqrt{6} H}, \\
Y & =\frac{\mu \phi}{2^{\frac{1}{n}} 3^{\frac{1}{2 n}}((3 \lambda-1) n)^{\frac{1}{2 n}} H^{\frac{1}{n}}}, \\
T & =\frac{c}{c+H^{\frac{1}{n}}},
\end{aligned}
$$

where $c=2^{\frac{3}{2}-\frac{1}{n}} 3^{\frac{n-1}{2 n}} n^{-\frac{1}{2 n}}(3 \lambda-1)^{-\frac{1}{2 n}} \mu$, such that

$$
\dot{\phi}=2 \sqrt{6} \Sigma c^{n}\left(\frac{1}{T}-1\right)^{n}
$$

$\phi=\frac{2^{\frac{1}{n}} 3^{\frac{1}{2 n}} n^{\frac{1}{2 n}} c\left(\frac{1}{T}-1\right) Y(3 \lambda-1)^{\frac{1}{2 n}}}{\mu}$,

$H=c^{n}\left(\frac{1}{T}-1\right)^{n}$,

and

$x=\Sigma$,

$y=Y^{n}$,

$z=\frac{\mu c^{-n}\left(\frac{1}{T}-1\right)^{-n}}{4(3 \lambda-1) a^{2}}$,

$s=\frac{n T}{\sqrt{6}(T-1) Y}$,

$Z=\frac{\mu c^{-n}\left(\frac{1}{T}-1\right)^{-n}}{\sqrt{16 a^{2}(1-3 \lambda)^{2}+\mu^{2} c^{-2 n}\left(\frac{1}{T}-1\right)^{-2 n}}}$,

$S=\frac{2 \arctan \left(\frac{n T}{\sqrt{6}(T-1) Y}\right)}{\pi}$.

and the new time variable $\frac{d \bar{\tau}}{d t}=H(1-T)^{-1}$. Therefore, we obtain the dynamical system

$\frac{d \Sigma}{d \bar{\tau}}=-n T Y^{2 n-1}-(2-q) \Sigma(1-T)$,

$\frac{d Y}{d \bar{\tau}}=\Sigma T-\frac{(q+1)(T-1) Y}{n}$,

$\frac{d T}{d \bar{\tau}}=\frac{(q+1)(T-1)^{2} T}{n}$,

where the fractional energy density of the scalar field energy density, $\Omega_{\phi}$, and the deceleration parameter, $q$ are:

$\Omega_{\phi}:=\Sigma+Y^{2 n}=1$,

$q=\frac{1}{2}\left(-3 Y^{2 n}+3 \Sigma^{2}+1\right)=-1+3 \Sigma^{2}$.

Introducing the complementary global transformation

$\Sigma=F(\theta) \sin (\theta), \quad Y=\cos (\theta)$,

$F(\theta)=\sqrt{\frac{1-\cos ^{2 n}(\theta)}{1-\cos ^{2}(\theta)}}=\sum_{k=0}^{n-1} \cos ^{2 k}(\theta)$,

we obtain the following regular unconstrained 2D dynamical system

$$
\begin{aligned}
& \frac{d \theta}{d \bar{\tau}}=-T F(\theta)-\frac{3(1-T) F(\theta)^{2} \sin (2 \theta)}{2 n}, \\
& \frac{d T}{d \bar{\tau}}=\frac{3(T-1)^{2} T\left(1-\cos ^{2 n}(\theta)\right)}{n},
\end{aligned}
$$

and the deceleration parameter satisfies 
Fig. 2 a Compact phase portrait of (32), (33), (34) for the choice $n=2$. b Dynamics in the invariant set $Z=0$. c Compact phase portrait of (54) and (56) for $-1<Y<0$. d Unwrapped solution space for the system (60), (61) for $n=1,4$

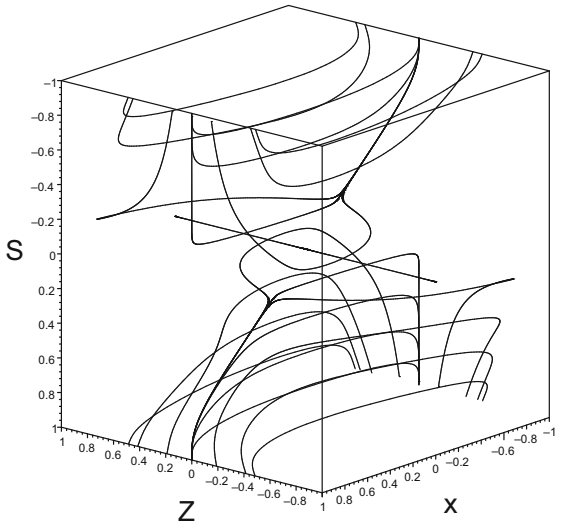

(a)
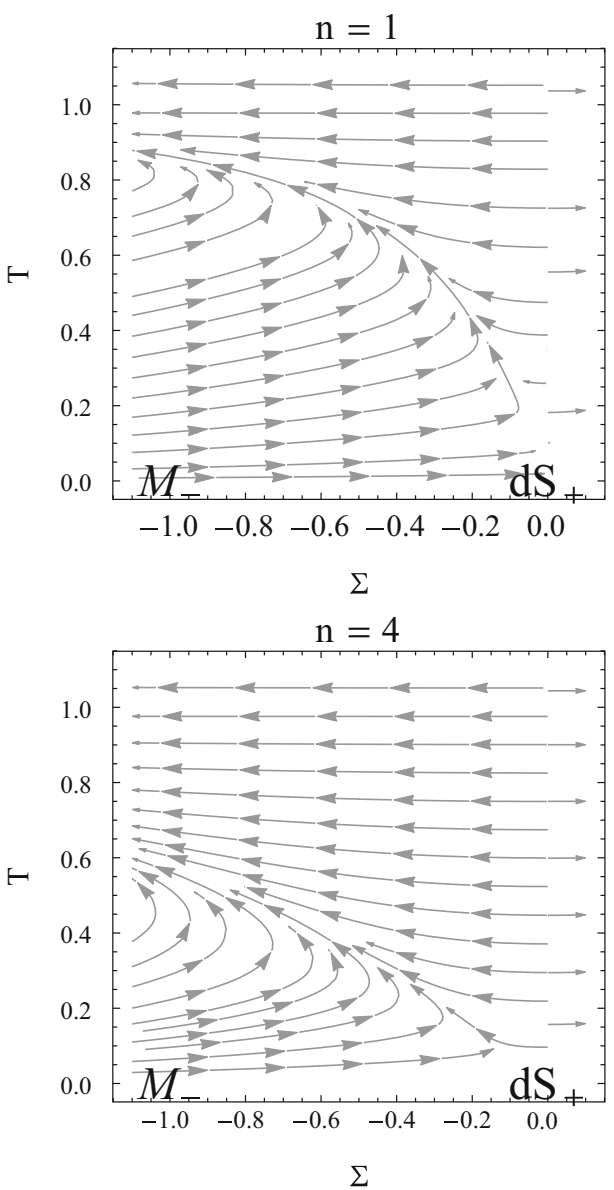

(c)

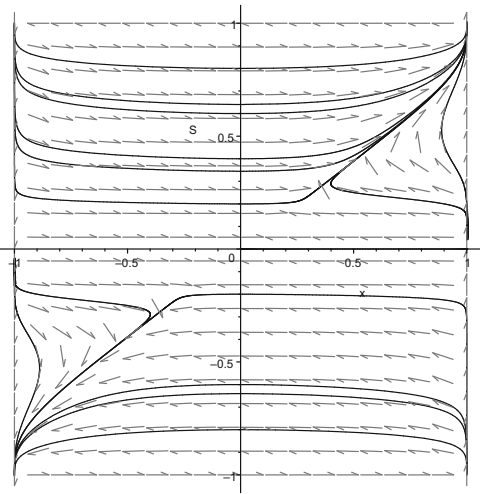

(b)
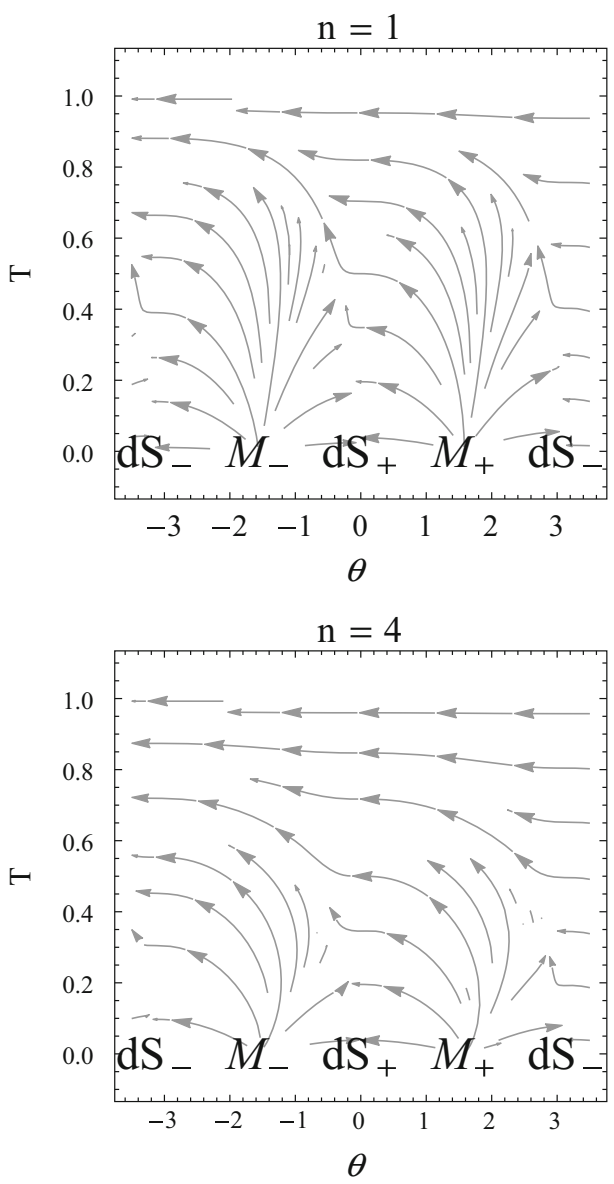

(d) $q=2-3 \cos ^{2 n}(\theta)$

We see that $F(\theta) \rightarrow \sqrt{n}$, as $\theta \rightarrow 0$. The dynamics on the invariant set $T=0$ is restricted to the set $\Sigma^{2}+Y^{2 n}=1$. The equilibrium points of the system (60), (61) are $M_{ \pm}=$ $(\Sigma, Y)=( \pm 1,0)$, corresponding to $\theta= \pm \frac{\pi}{2}$, representing massless scalar field cosmologies, and $d S_{ \pm}=(\Sigma, Y)=$ $(0, \pm 1)$, representing de Sitter solutions, corresponding to $\theta=0, \pi$ respectively. The following analysis is an specific application of the results discussed in [104] for the vac- uum case. The attractors at this boundary set (the scalar field boundary) are $d S_{ \pm}$and the sources are $M_{ \pm}$. The solutions starting near $M_{+}$approach the invariant set $T=1$ and tends to the limit-cycle given by the circle $\Sigma^{2}+Y^{2 n}, T=1$, that represents the Minkowski solution. In the Fig. 2 it is shown (a) a compact phase portrait of (32), (33), (34) for the choice $n=2$; (b) the dynamics in the invariant set $Z=0$; (c) a compact phase portrait of (54) and (56) for $-1<Y<0$, and (d) the unwrapped solution space of (60), (61) for $n=1,4$. 


\subsubsection{Stability analysis of the de Sitter solution in}

Hořava-Lifshitz cosmology for the flat universe with $\Lambda=0$ under the detailed-balance condition for the powerlaw-potential

To find the center manifold of the de Sitter solution $d S_{+}$it is convenient to define $\tilde{T}=\frac{T}{1-T}$, and consider $N=\ln \left(a / a_{0}\right)$ as the time variable, therefore, we find

$$
\begin{aligned}
& \frac{d \theta}{d N}=\frac{3 \tilde{T}\left(1-\cos ^{2 n}(\theta)\right)}{n}, \\
& \frac{d \tilde{T}}{d N}=-\tilde{T} F(\theta)-\frac{3 F^{2}(\theta) \sin (2 \theta)}{2 n} .
\end{aligned}
$$

Proposition 2 The origin for the system (63), (64) is unstable (saddle point).

Proof Taking the linear transformation

$(u, v)=\left(\tilde{T}, \frac{1}{3}(3 \theta+\sqrt{n} \tilde{T})\right)$,

and taking Taylor series near $(\theta, \tilde{T})=(0,0)$ up to fifth order we obtain the system (63), (64) can be written in diagonal form

$$
\begin{aligned}
\left(\begin{array}{l}
\frac{d u}{d N} \\
\frac{d v}{d N}
\end{array}\right)= & \left(\begin{array}{cc}
0 & 0 \\
0-\sqrt{n}
\end{array}\right)\left(\begin{array}{l}
u \\
v
\end{array}\right)+\left(\begin{array}{l}
f(u, v) \\
g(u, v)
\end{array}\right), \\
f(u, v)= & -\frac{1}{162} u\left((3 n-1)(\sqrt{n} u-3 v)^{2}-54\right) \\
& (\sqrt{n} u-3 v)^{2},
\end{aligned}
$$

and

$$
\begin{aligned}
& g(u, v)=-\frac{n^{3 / 2}(n(5 n(3 n+2)-441)+144) u^{4} v}{1944} \\
& +\frac{n^{2}\left(n\left(55 n^{2}+50 n-2201\right)+720\right) u^{5}}{116640} \\
& +\frac{1}{216} \sqrt{n} u^{2} v\left(24\left(n^{2}+n-18\right)-(5 n(n(7 n+2)-89)+144) v^{2}\right) \\
& +\frac{u^{3}\left(n(n(5 n(13 n+6)-1327)+432) v^{2}-4 n(n(3 n+5)-108)\right)}{1296} \\
& +\frac{1}{288} u\left((n(5 n(15 n+2)-453)+144) v^{4}-24(n+3)(5 n-12) v^{2}\right) \\
& +\frac{1}{30} \sqrt{n} v^{3}\left(-5 n\left(n v^{2}-3\right)+v^{2}+5\right),
\end{aligned}
$$

are nonlinear, vanish at 0 and have vanishing derivatives at 0 . By the Theorem 1, there exists a 1-dimensional invariant local center manifold $W^{c}(0)$ of $(66), W^{c}(0)=$ $\{(u, v) \in \mathbb{R} \times \mathbb{R}: v=h(u)\}$, satisfying $h(0)=0, h^{\prime}(0)=$ $0, \quad|u|<\delta$ for $\delta$ sufficiently small. According to Theorem 2 , the restriction of (66) to the center manifold is $\frac{d u}{d N}=f(u, h(u))$, where the function $h(u)$ that defines the local center manifold satisfies

$h^{\prime}(u)[f(u, h(u))]+\sqrt{n} h(u)-g(u, h(u))=0$.

According to Theorem 3, we can use Taylor series as follows $h(u)=a_{1} u^{2}+a_{2} u^{3}+a_{3} u^{4}+a_{4} u^{5}+\mathscr{O}(u)^{6}$, to obtain the nonzero coefficients

$$
\begin{aligned}
& a_{2}=-\frac{1}{324} \sqrt{n}(n(3 n+5)-108), \\
& a_{4}=\frac{\sqrt{n}\left(1080 n^{5 / 2}+1800 n^{3 / 2}-65 n^{4}-270 n^{3}+4079 n^{2}+8640 n-38880 \sqrt{n}-77760\right)}{116640} .
\end{aligned}
$$

$$
\begin{aligned}
\theta= & -\frac{\sqrt{n} \tilde{T}}{3}-\frac{1}{324}(\sqrt{n}(n(3 n+5)-108)) \tilde{T}^{3} \\
& +\frac{\sqrt{n}\left(1080 n^{5 / 2}+1800 n^{3 / 2}-65 n^{4}-270 n^{3}+4079 n^{2}+8640 n-38880 \sqrt{n}-77760\right) \tilde{T}^{5}}{116640}+O\left(\tilde{T}^{6}\right) .
\end{aligned}
$$


The dynamics on the center manifold can be approximated by the gradient-like equation

$$
\begin{aligned}
\frac{d u}{d N}= & -\nabla \Pi(u), \\
\Pi(u)= & \frac{n\left(9 n^{5 / 2}+15 n^{3 / 2}-n^{3}+6 n^{2}+99 n-324 \sqrt{n}-810\right) u^{8}}{3888} \\
& -\frac{1}{162}(n-18) n u^{6}-\frac{n u^{4}}{12}
\end{aligned}
$$

Due to the first nonzero derivative of $\Pi$ evaluated a $u=0$ is $\Pi^{(4)}(0)=-2 n<0$, it follows that $u=0$ is a degenerated maximum of the potential. Using the Theorem 2 , we conclude that the center manifold of origin for the system (63), (64), and the origin itself are unstable (saddle point). Therefore, the center manifold of $d S_{+}$is a good approximation for the early time attractor (see the reference [104], Section 4).

\subsection{E-models}

In this subsection we consider the E-model with potential $V(\phi)=V_{0}\left(1-e^{-\sqrt{\frac{2}{3 \alpha}} \phi}\right)^{2 n}$. This is a non-negative potential with a single minimum located at $(\phi, V(\phi))=(0,0)$. Therefore, the model admits an Minkowski solution represented by the equilibrium point $(H, \dot{\phi}, \phi)=(0,0,0)$. The potential has a plateau $V=V_{0}$, when $\phi \rightarrow+\infty$, while $V \sim V_{0} e^{-2 n \sqrt{\frac{2}{3 \alpha}} \phi}$ as $\phi \rightarrow-\infty$ [105]. At small $\phi$ the Epotential behaves as $\phi^{2 n}$.

For this choice we have

$$
\begin{aligned}
& f(s)=-\frac{s(s-\sqrt{6} \mu)}{2 n}, \quad \mu=\frac{2 n}{3 \sqrt{\alpha}}, \\
& f(s)=0 \Leftrightarrow s=0 \text { or } s=\sqrt{6} \mu, \\
& f^{\prime}(0)=\frac{\sqrt{\frac{3}{2}} \mu}{n}>0, \quad f^{\prime}(\sqrt{6} \mu)=-\frac{\sqrt{\frac{3}{2}} \mu}{n}<0 .
\end{aligned}
$$

The equations become

$$
\begin{aligned}
& \frac{d x}{d N}=\left(x^{2}-1\right)\left(3 x-\sqrt{6} \tan \left(\frac{\pi S}{2}\right)\right), \\
& \frac{d Z}{d N}=\left(2-3 x^{2}\right) Z\left(Z^{2}-1\right) \\
& \frac{d S}{d N}=\frac{x(-6 \mu \sin (\pi S)-\sqrt{6} \cos (\pi S)+\sqrt{6})}{\pi n},
\end{aligned}
$$

defined on the compact phase space

$$
\left\{(x, Z, S) \in \mathbb{R}^{3}:-1 \leq x \leq 1,-1 \leq Z \leq 1,-1 \leq S \leq 1\right\} .
$$

The equilibrium points of (71), (72), (73), and their stability conditions are summarized as follows.

- $(x, Z, S)=(-1,-1,0)$. The eigenvalues are $\left\{10,6, \frac{6 \mu}{n}\right\}$. It is a source.

- $(x, Z, S)=\left(-1,-1, \frac{2}{\pi} \arctan (\sqrt{6} \mu)\right)$. The eigenvalues are $\left\{10,12 \mu+6,-\frac{6 \mu}{n}\right\}$. It is a saddle.

$-(x, Z, S)=(-1,0,0)$. The eigenvalues are $\left\{-5,6, \frac{6 \mu}{n}\right\}$. It is a saddle.

- $(x, Z, S)=\left(-1,0, \frac{2}{\pi} \arctan (\sqrt{6} \mu)\right)$. The eigenvalues are $\left\{-5,12 \mu+6,-\frac{6 \mu}{n}\right\}$. It is a saddle.

- $(x, Z, S)=(-1,1,0)$. The eigenvalues are $\left\{6,10, \frac{6 \mu}{n}\right\}$. It is a source.

- $(x, Z, S)=\left(-1,1, \frac{2}{\pi} \arctan (\sqrt{6} \mu)\right)$. The eigenvalues are $\left\{10,12 \mu+6,-\frac{6 \mu}{n}\right\}$. Saddle.

- $(x, Z, S)=(0,-1,0)$. The eigenvalues are $\{4,-3,0\}$. Nonhyperbolic. Behaves as Saddle.

- $(x, Z, S)=(0,0,0)$. The eigenvalues are $\{-3,-2,0\}$. Nonhyperbolic.

$-(x, Z, S)=(0,1,0)$. The eigenvalues are $\{4,-3,0\}$. Nonhyperbolic. Behaves as a saddle.

$-(x, Z, S)=(1,-1,0)$. The eigenvalues are $\{-2,6$, $\left.-\frac{6 \mu}{n}\right\}$. Saddle.

- $(x, Z, S)=\left(1,-1, \frac{2}{\pi} \arctan (\sqrt{6} \mu)\right)$. The eigenvalues are $\left\{-2,6-12 \mu, \frac{6 \mu}{n}\right\}$. Saddle.

- $(x, Z, S)=(1,0,0)$. The eigenvalues are $\left\{1,6,-\frac{6 \mu}{n}\right\}$. Saddle.

- $(x, Z, S)=\left(1,0, \frac{2}{\pi} \arctan (\sqrt{6} \mu)\right)$. The eigenvalues are $\left\{1,6-12 \mu, \frac{6 \mu}{n}\right\}$. Source for $\mu<\frac{1}{2}$.

$-(x, Z, S)=(1,1,0)$. The eigenvalues are $\left\{-2,6,-\frac{6 \mu}{n}\right\}$. Saddle.

- $(x, Z, S)=\left(1,1, \frac{2}{\pi} \arctan (\sqrt{6} \mu)\right)$. The eigenvalues are $\left\{-2,6-12 \mu, \frac{6 \mu}{n}\right\}$. Saddle.

- $(x, Z, S)=\left(2 \mu,-1, \frac{2}{\pi} \arctan (\sqrt{6} \mu)\right)$. The eigenvalues are $\left\{4-48 \mu^{3}, 12 \mu^{2}-3, \frac{12 \mu^{2}}{n}\right\}$. Saddle.

- $(x, Z, S)=\left(2 \mu, 0, \frac{2}{\pi} \arctan (\sqrt{6} \mu)\right)$. The eigenvalues are $\left\{24 \mu^{3}-2,12 \mu^{2}-3, \frac{12 \mu^{2}}{n}\right\}$. Source for $n>0, \mu>$ $\frac{1}{2}$. Saddle otherwise.

- $(x, Z, S)=\left(2 \mu, 1, \frac{2}{\pi} \arctan (\sqrt{6} \mu)\right)$. The eigenvalues are $\left\{4-48 \mu^{3}, 12 \mu^{2}-3, \frac{12 \mu^{2}}{n}\right\}$. Saddle. 


\subsubsection{Stability analysis of the solution \\ $P_{3}^{0}:(x, Z, S)=(0,0,0)$ in Hořava-Lifshitz \\ cosmology for the flat universe with $\Lambda=0$ under the detailed-balance condition for the E-model}

Proposition 3 The origin for the system (71), (72), (73) is unstable (center-saddle).

Proof Taking the linear transformation

$\left(u, v_{1}, v_{2}\right)=\left(S, \frac{1}{6}(6 x-\sqrt{6} \pi S), Z\right)$,

and taking Taylor series near $\left(u, v_{1}, v_{2}\right)=(0,0,0)$ up to fifth order we obtain the system (71), (72), (73) can be written into its Jordan canonical form:

$$
\begin{aligned}
\left(\begin{array}{c}
\frac{d u}{d N} \\
\frac{d v_{1}}{d N} \\
\frac{d v_{2}}{d N}
\end{array}\right)= & \left(\begin{array}{ccc}
0 & 0 & 0 \\
0 & -3 & 0 \\
0 & 0 & -2
\end{array}\right)\left(\begin{array}{c}
u \\
v_{1} \\
v_{2}
\end{array}\right)+\left(\begin{array}{c}
f(u, \mathbf{v}) \\
g_{1}(u, \mathbf{v}) \\
g_{2}(u, \mathbf{v})
\end{array}\right), \\
f(u, \mathbf{v})= & -\frac{\pi^{4} u^{5}}{24 n}-\frac{\pi^{3} u^{4}\left(v_{1}-4 \mu\right)}{4 \sqrt{6} n}+\frac{\pi^{2} u^{3}\left(2 \mu v_{1}+1\right)}{2 n} \\
& +\frac{\sqrt{\frac{3}{2}} \pi u^{2}\left(v_{1}-2 \mu\right)}{n}-\frac{6 \mu u v_{1}}{n}, \\
g_{1}(u, \mathbf{v})= & \frac{\pi^{2} u^{2}\left(720 \mu+\pi u\left(\sqrt{6} \pi^{2}(5-2 n) u^{2}+30 \sqrt{6}(n-2)-120 \pi \mu u\right)\right)}{720 n} \\
& +\frac{\pi u v_{1}\left(24 \sqrt{6} \mu+\pi u\left(\pi^{2}(1-2 n) u^{2}+12(n-1)-4 \sqrt{6} \pi \mu u\right)\right)}{24 n} \\
& -\frac{\pi u\left(\pi^{2} u^{2}-24\right) v_{1}^{2}}{4 \sqrt{6}}+3 v_{1}^{3}, \\
g_{2}(u, \mathbf{v})= & \frac{1}{2} v_{2}\left(4 v_{2}^{2}-\left(v_{2}^{2}-1\right)\left(\pi^{2} u^{2}+2 \sqrt{6} \pi u v_{1}+6 v_{1}^{2}\right)\right) .
\end{aligned}
$$

According to Theorem 1, there exists a 1-dimensional invariant local center manifold $W^{c}(\mathbf{0})$ of $(75), W^{c}(\mathbf{0})=$ $\left\{(u, \mathbf{v}) \in \mathbb{R} \times \mathbb{R}^{2}: \mathbf{v}=\mathbf{h}(u)\right\}$, satisfying $\mathbf{h}(0)=\mathbf{0}$, $D \mathbf{h}(0)=\mathbf{0},|u|<\delta$ for $\delta$ sufficiently small. The restriction of (75) to the center manifold is $\frac{d u}{d N}=f(u, \mathbf{h}(u))$, where the function $\mathbf{h}(u)$ satisfies (A.10):

$$
D \mathbf{h}(u)[f(u, \mathbf{h}(u))]-P \mathbf{h}(u)-\mathbf{g}(u, \mathbf{h}(u))=0,
$$

where

$$
P=\left(\begin{array}{cc}
-3 & 0 \\
0 & -2
\end{array}\right) \text {. }
$$

According to Theorem 3, the system (76) can be solved approximately by expanding $\mathbf{h}(u)$ in Taylor series at $u=0$. Using the ansatsz

$$
\mathbf{h}(u):=\left(\begin{array}{l}
h_{1}(u) \\
h_{2}(u)
\end{array}\right)=\left(\begin{array}{c}
\sum_{j=1}^{4} a_{j} u^{j+1}+O\left(u^{6}\right) \\
\sum_{j=1}^{4} b_{j} u^{j+1}+O\left(u^{6}\right)
\end{array}\right),
$$

we find the non-null coefficients

$$
\begin{aligned}
& a_{1}=\frac{\pi^{2} \mu}{3 n}, a_{2}=\frac{\pi^{3}\left(24 \mu^{2}+(n-2) n\right)}{12 \sqrt{6} n^{2}}, \\
& a_{3}=\frac{\pi^{4} \mu\left(56 \mu^{2}+n(2 n-7)\right)}{18 n^{3}}, \\
& a_{4}=\frac{\pi^{5}\left(13600 \mu^{4}+n^{2}(n(3 n-25)+40)+20 \mu^{2} n(29 n-108)\right)}{360 \sqrt{6} n^{4}} .
\end{aligned}
$$

Therefore, the center manifold can be represented locally by the graph

$$
\begin{aligned}
v_{1} & =\frac{\pi^{2} \mu u^{2}}{3 n}+\frac{\pi^{3}\left(24 \mu^{2}+(n-2) n\right) u^{3}}{12 \sqrt{6} n^{2}} \\
& +\frac{\pi^{4} \mu\left(56 \mu^{2}+n(2 n-7)\right) u^{4}}{18 n^{3}} \\
& +\frac{\pi^{5}\left(13600 \mu^{4}+20 n(29 n-108) \mu^{2}+n^{2}(n(3 n-25)+40)\right) u^{5}}{360 \sqrt{6} n^{4}}, \\
v_{2} & =0 .
\end{aligned}
$$

That is,

$$
\begin{aligned}
x= & \frac{\pi S}{\sqrt{6}}+\frac{\pi^{2} \mu S^{2}}{3 n}+\frac{\pi^{3}\left(24 \mu^{2}+(n-2) n\right) S^{3}}{12 \sqrt{6} n^{2}} \\
& +\frac{\pi^{4} \mu\left(56 \mu^{2}+n(2 n-7)\right) S^{4}}{18 n^{3}} \\
& +\frac{\pi^{5}\left(13600 \mu^{4}+20 n(29 n-108) \mu^{2}+n^{2}(n(3 n-25)+40)\right) S^{5}}{360 \sqrt{6} n^{4}}, \\
Z & =0 .
\end{aligned}
$$

The dynamics on the center manifold is given by the gradientlike equation

$$
\begin{aligned}
\frac{d u}{d N}= & -\nabla \Pi(u), \\
\Pi(u)= & \frac{\sqrt{\frac{2}{3}} \pi \mu u^{3}}{n}-\frac{\pi^{2} u^{4}\left(n-4 \mu^{2}\right)}{8 n^{2}} \\
& -\frac{\pi^{3} \mu u^{5}\left(n(n+4)-24 \mu^{2}\right)}{10 \sqrt{6} n^{3}}+\frac{\pi^{4} u^{6}\left(224 \mu^{4}+n^{2}+4 \mu^{2}(n-10) n\right)}{72 n^{4}} \\
& -\frac{\pi^{5} \mu u^{7}\left(-6800 \mu^{4}+n^{2}(n(n+15)-55)+10 \mu^{2}(136-23 n) n\right)}{210 \sqrt{6} n^{5}} .
\end{aligned}
$$

We have $\Pi^{\prime}(0)=\Pi^{\prime \prime}(0)=0, \Pi^{\prime \prime \prime}(0)=\frac{2 \sqrt{6} \pi \mu}{n}$. It follows that $u=0$ is an inflection of the potential. Using the Theorem 2, we conclude that the center manifold of origin for the system (75), and the origin are unstable (center-saddle).

\subsubsection{Alternative compactification}

As we have commented before, $V(\phi)=V_{0}\left(1-e^{-\sqrt{\frac{2}{3 \alpha}} \phi}\right)^{2 n}$ is a non-negative potential with a single minimum located at $(\phi, V(\phi))=(0,0)$, with $(H, \dot{\phi}, \phi)=(0,0,0)$, corresponding to the Minkowski solution. The potential has a plateau $V=V_{0}$, when $\phi \rightarrow+\infty$, while $V \sim V_{0} e^{-2 n \sqrt{\frac{2}{3 \alpha}} \phi}$ as 
$\phi \rightarrow-\infty$ [105]. At small $\phi$ the E-potential behaves as $\phi^{2 n}$, so, it can be implemented a similar approach as the one used for $\sim \phi^{2 n}$ potentials in Sect. 3.3. That is, we define new variables given by

$\Sigma=\frac{\dot{\phi}}{2 \sqrt{6} H}$,

$Y=\left(\frac{V(\phi)}{6(3 \lambda-1) H^{2}}\right)^{\frac{1}{2 n}}=\tilde{T}\left(1-e^{-\sqrt{\frac{2}{3 \alpha}} \phi}\right)$,

$\tilde{T}=\left[\frac{V_{0}}{6(3 \lambda-1) H^{2}}\right]^{\frac{1}{2 n}}$,

such that

$$
\begin{aligned}
\dot{\phi} & =\frac{2 \Sigma \sqrt{V_{0}} \tilde{T}^{-n}}{\sqrt{3 \lambda-1}}, \\
\phi & =-\frac{\sqrt{\frac{2}{3}} n \ln \left(1-\frac{Y}{\tilde{T}}\right)}{\mu}, \\
H & =\frac{\sqrt{V_{0}} \tilde{T}^{-n}}{\sqrt{3(6 \lambda-2)}},
\end{aligned}
$$

and

$$
x=\Sigma,
$$$$
y=Y^{n},
$$

$z=\frac{\sqrt{\frac{3}{2}} \mu \tilde{T}^{n}}{2 \sqrt{3 \lambda-1} \sqrt{V_{0}} a^{2}}$,

$u=\frac{\sqrt{\frac{3}{2}} \Lambda \mu \tilde{T}^{n}}{2 \sqrt{3 \lambda-1} \sqrt{V_{0}}}$,

$s=\sqrt{6} \mu\left(1-\frac{\tilde{T}}{Y}\right)$,

$Z=\frac{\sqrt{3} \mu \tilde{T}^{n}}{\sqrt{24 \lambda V_{0} a^{4}-8 V_{0} a^{4}+3 \mu^{2} \tilde{T}^{2 n}}}$,

$S=\frac{2 \arctan \left(\sqrt{6} \mu\left(1-\frac{\tilde{T}}{Y}\right)\right)}{\pi}$,

where the fractional energy density of the scalar field energy density, $\Omega_{\phi}$, and the deceleration parameter, $q$ are:

$$
\begin{aligned}
& \Omega_{\phi}:=\Sigma^{2}+Y^{2 n}=1, \\
& q=\frac{1}{2}\left(-3 Y^{2 n}+3 \Sigma^{2}+1\right)=-1+3 \Sigma^{2} .
\end{aligned}
$$

We obtain the dynamical system:

$$
\frac{d \Sigma}{d N}=6 \mu Y^{2 n-1}(Y-\tilde{T})+(q-2) \Sigma,
$$

$$
\begin{aligned}
& \frac{d Y}{d N}=\frac{Y(-6 \mu \Sigma+q+1)+6 \mu \Sigma \tilde{T}}{n}, \\
& \frac{d \tilde{T}}{d N}=\frac{(q+1) \tilde{T}}{n} .
\end{aligned}
$$

This system have been extensively studied in [105] in the context of a canonical scalar field cosmology. Now, we will discuss the more relevant features of solutions space. It can be easily proven that $\tilde{T}$ is monotonically increasing toward the future and decreasing towards the past. The phase space is limited to the past by the invariant subset $\tilde{T}=0$, for $Y \leq 0$, and by $\tilde{T}-Y=0$, for $Y \geq 0$. The state space is bounded when $\tilde{T}>0, \tilde{T}-Y>0$. As for the monomial potential with odd exponent, the two past boundaries are intersected at the two massless scalar field points $M_{ \pm}=(\Sigma, Y)=$ $( \pm 1,0)$. The subset $\tilde{T}-Y=0$, on the other hand, it is divided in two disconnected regions separated by the de Sitter equilibrium point $d S=(\tilde{T}, Y)=(1,1)$. This feature it is illustrated in the Fig. 3, where it is seen the phase-space has two disconnected regions, in different coordinates.

Introducing the complementary global transformation

$\Sigma=F(\theta) \sin (\theta), \quad Y=\cos (\theta)$,

$F(\theta)=\sqrt{\frac{1-\cos ^{2 n}(\theta)}{1-\cos ^{2}(\theta)}}$,

we obtain the following regular unconstrained 2D dynamical system

$$
\begin{aligned}
\frac{d \theta}{d N} & =-\frac{6 \mu F(\theta)(\tilde{T}-\cos (\theta))}{n}-\frac{3 F(\theta)^{2} \sin (2 \theta)}{2 n}, \\
\frac{d \tilde{T}}{d N} & =\frac{3 \tilde{T}\left(1-\cos ^{2 n}(\theta)\right)}{n}
\end{aligned}
$$

and the deceleration parameter becomes

$q=2-3 \cos ^{2 n}(\theta)$.

Introducing the new compact variable $T=\frac{\tilde{T}}{1+\tilde{T}}$ and the new time derivative $\frac{d \bar{\tau}}{d \ln a}=1+\tilde{T}=(1-T)^{-1}$, we obtain the regular system:

$$
\begin{aligned}
\frac{d \theta}{d \bar{\tau}}= & \frac{3(T-1) F(\theta)^{2} \sin (\theta) \cos (\theta)}{n} \\
& -\frac{6 \mu F(\theta)((T-1) \cos (\theta)+T)}{n}, \\
\frac{d T}{d \bar{\tau}}= & -\frac{3(T-1)^{2} T\left(\cos ^{2 n}(\theta)-1\right)}{n} .
\end{aligned}
$$

The past boundary is attached to the phase-space, and in the new variables $(\theta, T)$ it is defined by $\{T=0, \cos (\theta) \leq$ $0\} \cup\{T-(1-T) \cos \theta=0, \cos (\theta)>0\}$. It is also included the future boundary $T=1$, which corresponds to $H=$ 
Fig. 3 a Compact phase portrait of $(71),(72),(73)$ for the choice $n=2, \mu=1$. $\mathbf{b}$ Dynamics in the invariant set $Z=0$. c Unwrapped solution space (103), (104) for some values of $n, \mu, \alpha$

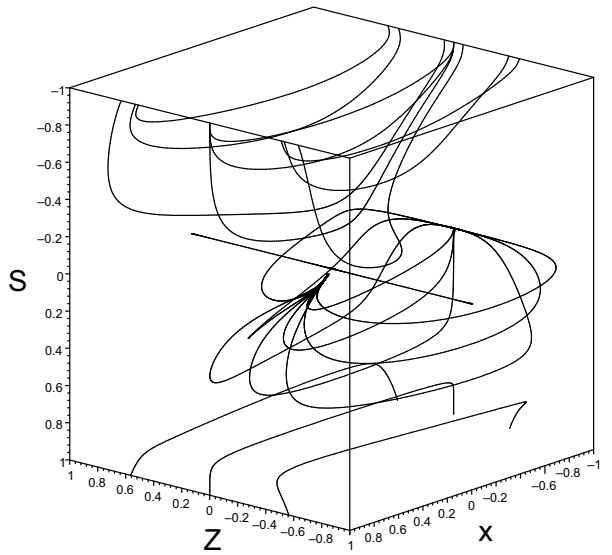

(a)
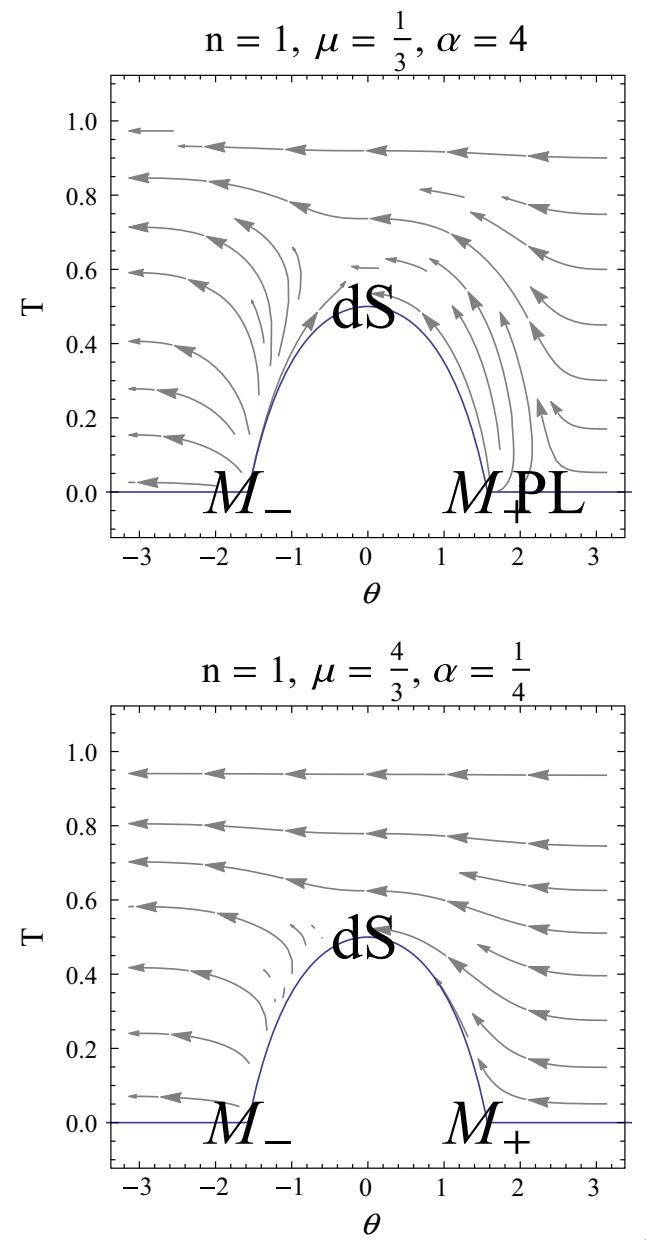

(c)

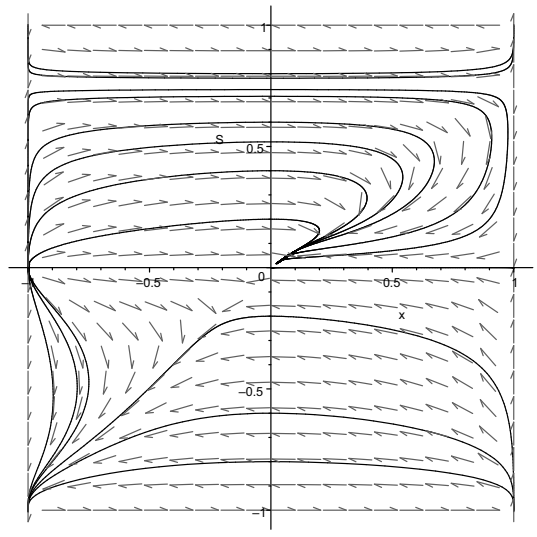

(b)
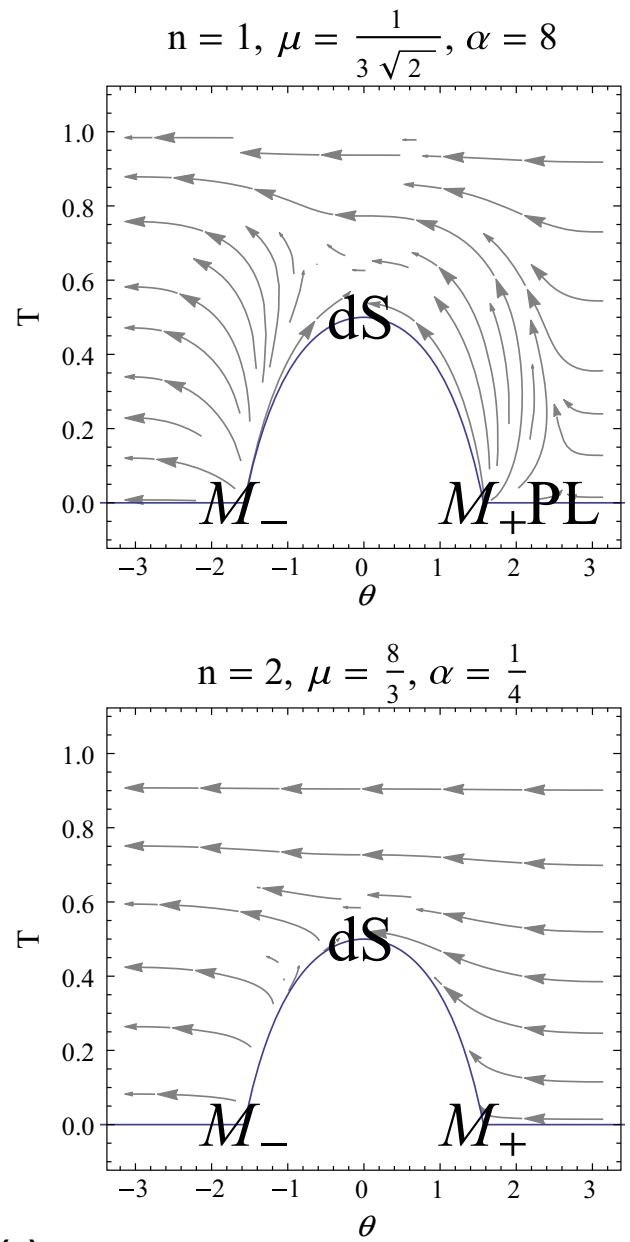

0 and the final state is the Minkowski point. The region $\{T-(1-T) \cos \theta<0, \cos (\theta)>0\}$ is forbidden.

The equilibrium points of (103), (104) are given by

- $M_{ \pm}: \tilde{T}=T=0 ; \Sigma= \pm 1, Y=0 ; \theta= \pm \frac{\pi}{2}+2 k \pi, k=$ $0,1,2 \ldots$ They are massless scalar field solutions. In the original variables these solutions corresponds to $P_{1,2}(\sqrt{6} \mu)$ :

$$
\left(x= \pm 1, y=0, z=0, s=\sqrt{6} \mu ; S=\frac{2 \arctan (\sqrt{6} \mu)}{\pi}\right) .
$$


They are saddle and source, respectively, as it is confirmed in Fig. 3c where its presented the unwrapped solution space of (103), (104) for some values of $n, \mu, \alpha$.

- $d S: \tilde{T}=1, T=\frac{1}{2} ; \Sigma=0, Y=1 ; \theta=2 k \pi, k=$ $0,1,2 \ldots$ It is a de Sitter solution. In the original variables the solution $d S$ corresponds to the point $(x=$ $0, y=1, z=0 ; s=0, S=0)$, i.e, it is represented by $P_{3}^{0}:(x, Z, S)=(0,0,0)$, which is is nonhyperbolic since $f(0)=0$.

- PL: $\tilde{T}=T=0 ; \Sigma=2 \mu ; Y=-\left(1-4 \mu^{2}\right)^{\frac{1}{2 n}} ; \theta=$ $\pm \arccos Y$. Exists for $\mu<1 / 2$. It corresponds to a Powerlaw selfsimilar solution for the exponential potential. It is equivalent to $P_{3}(\sqrt{6} \mu)$.

In Fig. 3a it is shown a compact phase portrait of the system (71), (72), (73) for the choice $n=2, \mu=1$. In (b) it is shown the dynamics in the invariant set $Z=0$. In (c) it is represented the unwrapped solution space of the system (103), (104) for some values of $n, \mu, \alpha$. This plot clearly shows that the future boundary is $T=1$, which corresponds to $H=0$ and the final state is the Minkowski point given by a limit cycle.

\subsubsection{Stability analysis of the de Sitter solution in}

Horava-Lifshitz cosmology for the Flat universe with $\Lambda=0$ under the detailed-balance condition for the E-potential

In order to analyze the stability of de Sitter solution we can use center manifold theorem. It is more convenient to use the variables $(\theta, \tilde{T})$ whose evolution is given by the regular unconstrained 2D dynamical system (100), (101).

Proposition 4 The de Sitter solution $d S_{+}$, with $\theta=0$, for the system (100), (101) is unstable (center-saddle).

Proof Taking the linear transformation

$(u, v)=\left(\tilde{T}-1, \theta+\frac{2 \mu(\tilde{T}-1)}{\sqrt{n}}\right)$, and truncating the Taylor series at fifth order we obtain that the system (100), (101) can be written in diagonal form

$$
\begin{aligned}
&\left(\begin{array}{c}
\frac{d u}{d N} \\
\frac{d v}{d N}
\end{array}\right)=\left(\begin{array}{cc}
0 & 0 \\
0 & -3
\end{array}\right)\left(\begin{array}{c}
u \\
v
\end{array}\right)+\left(\begin{array}{c}
f(u, v) \\
g(u, v)
\end{array}\right), \\
& f(u, v)=\frac{4 \mu^{2} u^{3}\left(3 \sqrt{n}\left((1-3 n) v^{2}+1\right)+4 \mu(3 n-1) v\right)}{n^{3 / 2}} \\
& \quad+\frac{8 \mu^{4}(1-3 n) u^{5}}{n^{2}}+\frac{8 \mu^{3}(3 n-1) u^{4}(2 \sqrt{n} v-\mu)}{n^{2}} \\
& \quad+\frac{4 \mu u^{2}\left(3 \mu\left((1-3 n) v^{2}+1\right)+\sqrt{n} v\left((3 n-1) v^{2}-3\right)\right)}{n} \\
&++\frac{u v\left(5 \sqrt{n} v\left((1-3 n) v^{2}+6\right)-8 \mu\left((5-15 n) v^{2}+2 v^{4}+15\right)\right)}{10 \sqrt{n}} \\
&+\frac{1}{2}\left((1-3 n) v^{4}+6 v^{2}\right) \text { and } g(u, v) \\
&=\frac{\mu^{5}(5 n(11 n-38)+39) u^{5}}{5 n^{5 / 2}} \\
&-\frac{2 \mu^{4} u^{4}(-4 \mu+18 \mu n+\sqrt{n}(3 n-7)(5 n-1) v)}{n^{5 / 2}} \\
&+\frac{\mu^{2} u^{2}\left(12 \mu\left((2-9 n) v^{2}+2\right)+(1-n) \sqrt{n} v\left((35 n-3) v^{2}-24\right)\right)}{2 n^{3 / 2}} \\
&+\frac{\mu^{3} u^{3}\left(\sqrt{n}\left((n(65 n-114)+17) v^{2}-12 n+28\right)+16 \mu(9 n-2) v\right)}{2 n^{2}} \\
&+\frac{\mu u v\left(32 \mu\left((9 n-2) v^{2}-6\right)+\sqrt{n} v\left((n(75 n-38)-5) v^{2}-120 n+24\right)\right)}{16 n} \\
&+\frac{v^{2}\left(5 \mu\left((2-9 n) v^{2}+12\right)+2 \sqrt{n} v\left(-5 n\left(n v^{2}-3\right)+v^{2}+5\right)\right)}{20 \sqrt{n}} .
\end{aligned}
$$

According to Theorem 1, there exists a 1-dimensional invariant local center manifold of (106), $W^{c}(\mathbf{0})=\left\{(u, v) \in \mathbb{R}^{2}\right.$ : $v=h(u)\}$, where $h(0)=0, h^{\prime}(0)=0,|u|<\delta$ for $\delta$ sufficiently small. The restriction of (106) to the center manifold is $\frac{d u}{d N}=f(u, h(u))$, such that the function that defines the center manifold satisfies

$h^{\prime}(u)[f(u, h(u))]+3 h(u)-g(u, h(u))=0$.

According to Theorem 3, we can use Taylor series as follows $h(u)=a_{1} u^{2}+a_{2} u^{3}+a_{3} u^{4}+a_{4} u^{3}+\mathscr{O}(u)^{6}$, to obtain $a_{1}=$ $\frac{4 \mu^{3}}{n^{3 / 2}}, a_{2}=-\frac{2 \mu^{3}\left(72 \mu^{2}+n(3 n-7)\right)}{3 n^{5 / 2}}, a_{3}=\frac{12 \mu^{5}\left(76 \mu^{2}+n(3 n-10)\right)}{n^{7 / 2}}$, $a_{4}=\frac{-338880 \mu^{9}+\mu^{5} n^{2}\left(-65 n^{2}+570 n-1081\right)+120 \mu^{7}(459-121 n) n}{15 n^{9 / 2}}$. The center manifold can be expressed, in terms of $\tilde{T}$, by

$$
\begin{aligned}
\theta= & -\frac{2 \mu(\tilde{T}-1)}{\sqrt{n}}+\frac{4 \mu^{3}(\tilde{T}-1)^{2}}{n^{3 / 2}}+\frac{12 \mu^{5}(\tilde{T}-1)^{4}\left(76 \mu^{2}+n(3 n-10)\right)}{n^{7 / 2}}-\frac{2 \mu^{3}(\tilde{T}-1)^{3}\left(72 \mu^{2}+n(3 n-7)\right)}{3 n^{5 / 2}} \\
& +\frac{(\tilde{T}-1)^{5}\left(-338880 \mu^{9}+\mu^{5} n^{2}\left(-65 n^{2}+570 n-1081\right)+120 \mu^{7}(459-121 n) n\right)}{15 n^{9 / 2}} .
\end{aligned}
$$


Table 3 Case 2: Equilibrium points at the finite region of the system (113), (114), (115)

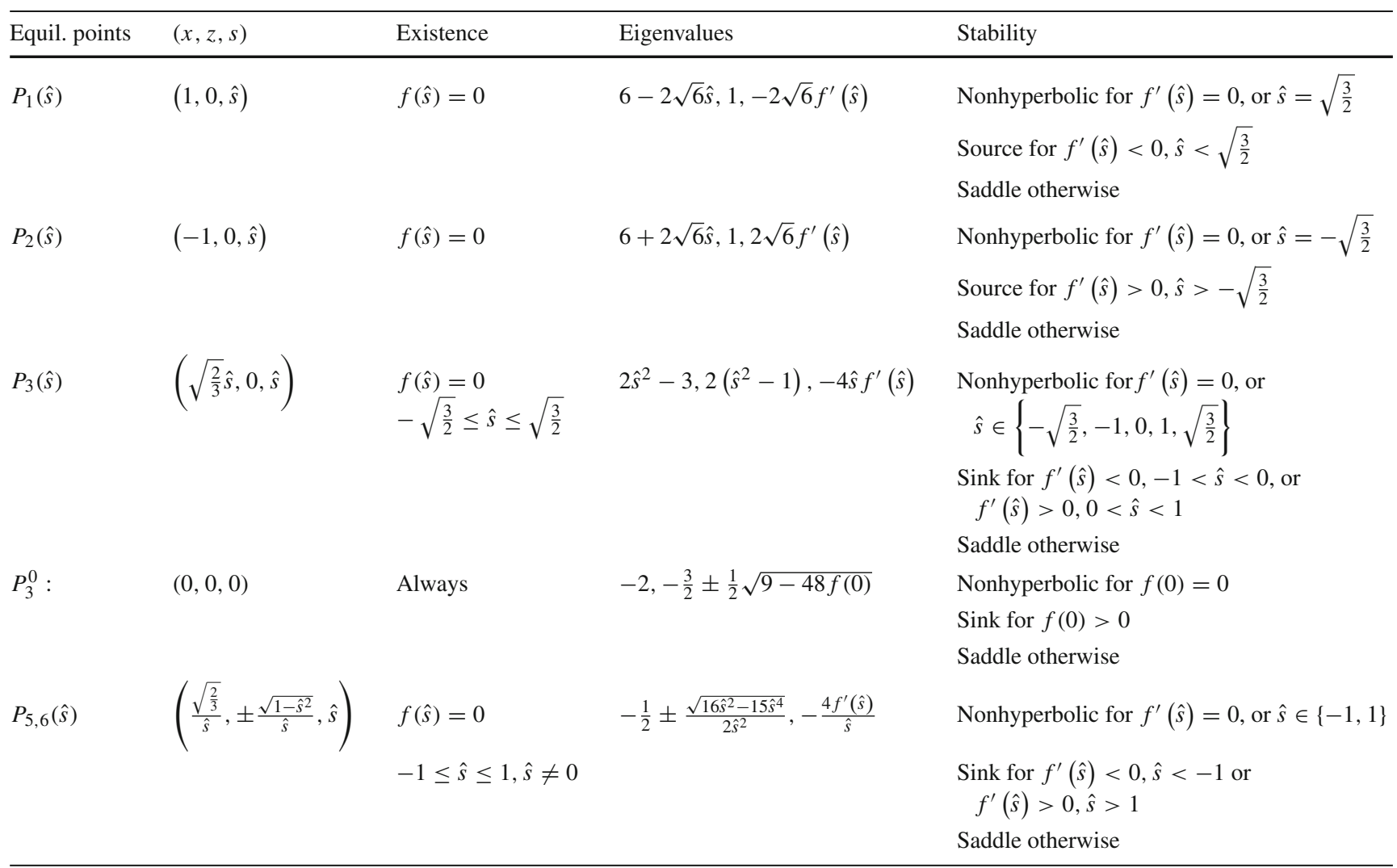

The dynamics on the center manifold can be approximated by the gradient-like equation

$$
\begin{aligned}
\frac{d u}{d N} & =-\nabla \Pi(u), \\
\Pi(u) & =-\frac{4 \mu^{2} u^{3}}{n}-\frac{3 \mu^{2} u^{4}\left(n-4 \mu^{2}\right)}{n^{2}}+\frac{48 \mu^{4} u^{5}\left(2 n-13 \mu^{2}\right)}{5 n^{3}} \\
& +\frac{8 \mu^{4} u^{6}\left(252 \mu^{4}+n^{2}+2 \mu^{2} n(3 n-22)\right)}{n^{4}} \\
& +\frac{48 \mu^{6} u^{7}\left(-6248 \mu^{4}+n^{2}(12 n-49)+2 \mu^{2}(619-106 n) n\right)}{7 n^{5}},
\end{aligned}
$$

with $\Pi^{\prime \prime \prime}(u)=-\frac{24 \mu^{2}}{n}<0$, that is, the origin is an inflection point. Using the Theorem 2, we conclude that the center manifold of origin for the system (106), and therefore $d S_{+}$ is unstable (center-saddle) (see [104], Section 4).

\section{Case 2: Non-flat universe with $\Lambda=0$ under the detailed-balance condition}

For this case the equations are:

$$
H^{2}=\frac{1}{6(3 \lambda-1)}\left[\frac{3 \lambda-1}{4} \dot{\phi}^{2}+V(\phi)\right]-\frac{\mu^{2} k^{2}}{16(3 \lambda-1)^{2} a^{4}},
$$

$8 \dot{H}+\dot{\phi}^{2}=\frac{k^{2} \mu^{2}}{(3 \lambda-1)^{2} a^{4}}$,

$\ddot{\phi}+3 H \dot{\phi}+\frac{2 V^{\prime}(\phi)}{3 \lambda-1}=0$.

\subsection{Arbitrary potential}

In this example the system (110), (111), (112), is reduced to the autonomous form:

$\frac{d x}{d N}=x\left(3 x^{2}-2 z^{2}-3\right)+\sqrt{6} s\left(1-x^{2}+z^{2}\right)$,

$\frac{d z}{d N}=z\left[3 x^{2}-2\left(z^{2}+1\right)\right]$,

$\frac{d s}{d N}=-2 \sqrt{6} x f(s)$.

defined on the phase space $\left\{(x, z, s) \in \mathbb{R}^{3}: x^{2}-z^{2} \leq 1\right\}$.

The equilibrium points/curves of the system (113), (114), (115), at the finite region of the phase space is presented in Table 3 . Now we discuss the more relevant features of them.

- $P_{1}(\hat{s}):(x, z, s)=(1,0, \hat{s})$. Always exists. It is a source for $f^{\prime}(\hat{s})<0, \hat{s}<\sqrt{\frac{3}{2}}$. 
Table 4 Case 2: Equilibrium points at the infinity region of the system (113), (114), (115)

\begin{tabular}{|c|c|c|c|c|}
\hline Equil. points & $(x, Z, S)$ & Existence & Eigenvalues & Stability \\
\hline$Q_{9,10}(\hat{s})$ & $\left(-1, \pm 1, \frac{2}{\pi} \arctan (\hat{s})\right)$ & $f(\hat{s})=0$ & $-\infty, 2 \sqrt{6} \hat{s}, 2 \sqrt{6} f^{\prime}(\hat{s})$ & Sinks for $\hat{s}<0, f^{\prime}(\hat{s})<0$ \\
\hline$Q_{11,12}(\hat{s})$ & $\left(1, \pm 1, \frac{2}{\pi} \arctan (\hat{s})\right)$ & $f(\hat{s})=0$ & $-\infty,-2 \sqrt{6} \hat{s},-2 \sqrt{6} f^{\prime}(\hat{s})$ & Sinks for $\hat{s}>0, f^{\prime}(\hat{s})>0$ \\
\hline$Q_{13,14}$ & $(0, \pm 1,0)$ & Always & $\infty,-2 \sqrt{3} \sqrt{-f(0)}, 2 \sqrt{3} \sqrt{-f(0)}$ & $\begin{array}{l}\text { Saddle for } f(0)<0 \text {. } \\
\text { Center for } f(0)>0\end{array}$ \\
\hline$Q_{15,16}$ & $\left(-\sqrt{\frac{3}{2}}, \pm 1,0\right)$ & $f(0)=0$ & $0,0,4 f^{\prime}(0)$ & Nonhyperbolic \\
\hline$Q_{17,18}$ & $\left(\sqrt{\frac{3}{2}}, \pm 1,0\right)$ & $f(0)=0$ & $0,0,-4 f^{\prime}(0)$ & Nonhyperbolic \\
\hline
\end{tabular}

- $P_{2}(\hat{s}):(x, z, s)=(-1,0, \hat{s})$. Always exists. It is a source for $f^{\prime}(\hat{s})>0, \hat{s}>-\sqrt{\frac{3}{2}}$.

$-P_{3}(\hat{s}):(x, z, s)=\left(\sqrt{\frac{2}{3}} \hat{s}, 0, \hat{s}\right)$. Exists for $-\sqrt{\frac{3}{2}} \leq \hat{s} \leq$ $\sqrt{\frac{3}{2}}$. It is a sink for $f^{\prime}(\hat{s})<0,-1<\hat{s}<0$, or $f^{\prime}(\hat{s})>$ $0,0<\hat{s}<1$.

$-P_{3}^{0}:(x, z, s)=(0,0,0)$. It is a sink for $f(0)>0$.

$-P_{5,6}(\hat{s}):\left(\frac{\sqrt{\frac{2}{3}}}{\hat{s}}, \pm \frac{\sqrt{1-\hat{s}^{2}}}{\hat{s}}, \hat{s}\right)$. Exists for $-1 \leq \hat{s} \leq 1, \hat{s} \neq$ 0 . It is a sink for $f^{\prime}(\hat{s})<0, \hat{s}<-1$ or $f^{\prime}(\hat{s})>0, \hat{s}>1$.

- There are two lines of equilibrium points $P_{7,8}\left(s_{c}\right)$ : $(x, z, s)=\left(0, \pm i, s_{c}\right), s_{c} \in \mathbb{R}$ which are not considered since they are complex valued.

Owing to the fact that the dynamical system (113), (114), (115) is unbounded, we introduce the new variables

$X=\frac{x}{\sqrt{1+z^{2}}}, \quad Z=\frac{z}{\sqrt{1+z^{2}}}, \quad S=\frac{2}{\pi} \arctan (s)$,

and the time rescaling

$\frac{d f}{d \tau}=\sqrt{1-Z^{2}} \frac{d f}{d N}$,

to obtain the dynamical system

$\frac{d X}{d \tau}=\left(X^{2}-1\right)\left(3 X \sqrt{1-Z^{2}}-\sqrt{6} \tan \left(\frac{\pi S}{2}\right)\right)$,

$\frac{d Z}{d \tau}=\left(3 X^{2}-2\right) Z \sqrt{1-Z^{2}}$,

$\frac{d S}{d \tau}=-\frac{2 \sqrt{6} X(\cos (\pi S)+1) f\left(\tan \left(\frac{\pi S}{2}\right)\right)}{\pi}$,

defined on the compacted phase space

$\left\{(X, Z, S) \in \mathbb{R}^{3}:-1 \leq X \leq 1,-1 \leq Z \leq 1,-1 \leq S \leq 1\right\}$.

The equilibrium points at the infinity region of the system (113), (114), (115) are summarized in Table 4. Here we discuss the main features of them.
- $Q_{9,10}(\hat{s}):(X, Z, S)=\left(-1, \pm 1, \frac{2}{\pi} \arctan (\hat{s})\right)$, where $f(\hat{s})=0$. They are sinks for $\hat{s}<0, f^{\prime}(\hat{s})<0$

- $Q_{11,12}(\hat{s}):(X, Z, S)=\left(1, \pm 1, \frac{2}{\pi} \arctan (\hat{s})\right)$, where $f(\hat{s})=0$. They are sinks for $\hat{s}>0, f^{\prime}(\hat{s})>0$.

- $Q_{13,14}:(X, Z, S)=(0, \pm 1,0)$. Saddle for $f(0)<0$. Center if $f(0)>0$.

- $Q_{15,16}:(X, Z, S)=\left(-\sqrt{\frac{3}{2}}, \pm 1,0\right)$, where $f(0)=0$. It is nonhyperbolic.

- $Q_{17,18}:(X, Z, S)=\left(\sqrt{\frac{3}{2}}, \pm 1,0\right)$, where $f(0)=0$. It is nonhyperbolic.

\subsection{Exponential potential}

In this case the system (118), (119), (120), becomes

$$
\begin{aligned}
& \frac{d X}{d \tau}=\left(X^{2}-1\right)\left(3 X \sqrt{1-Z^{2}}-\sqrt{6} s\right) \\
& \frac{d Z}{d \tau}=\left(3 X^{2}-2\right) Z \sqrt{1-Z^{2}}
\end{aligned}
$$

defined on the compact phase space

$\left\{(X, Z) \in \mathbb{R}^{2}:-1 \leq X \leq 1,-1 \leq Z \leq 1\right\}$.

The equilibrium points $P_{1,2,3}$ are exactly the same as in case 1 , and thus the physical implications are the same. The equilibrium points $P_{5,6}$ are unstable, corresponding to a darkmatter dominated universe. This was expected since in the absence of the cosmological constant $\Lambda$, the curvature role is downgrading as the scale factor increases and thus in the end this case tends to the case 1 above. Note however that at early times, where the scale factor is small, the behavior of the system will be significantly different than case 1, with the dark energy playing an important role. Figure 4a illustrates when $Q_{9,10}:(X, Z)=(-1, \pm 1)$ are sinks for $s<0$. Figure 4b-d illustrates when $Q_{11,12}:(X, Z)=(1, \pm 1)$ are sinks for $s>0$. 
Fig. 4 Compact phase portrait of (121), (122) for different choices of the parameter $s$
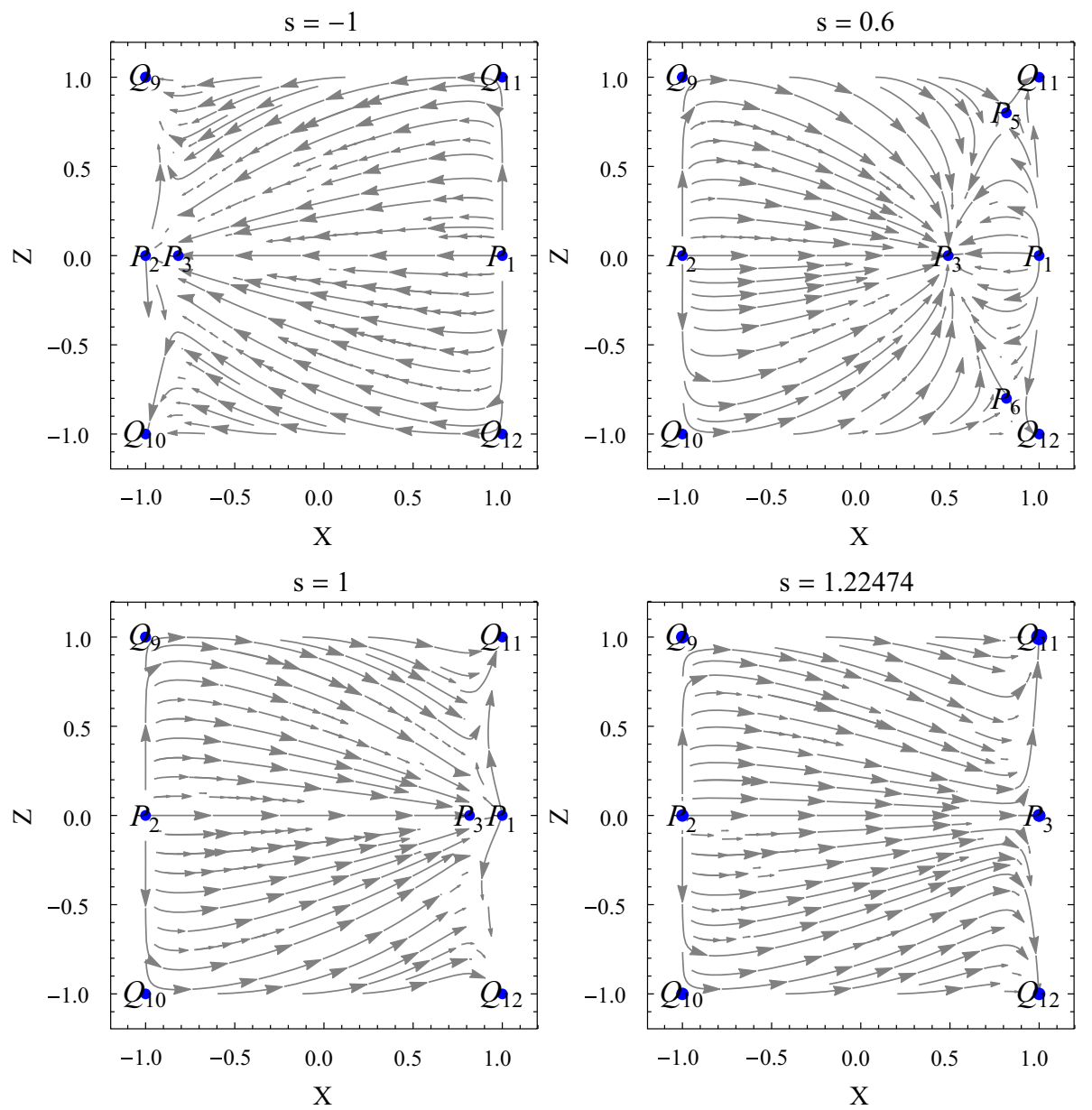

\subsection{Powerlaw potential}

In this example, the system (118), (119), (120), becomes

$$
\begin{aligned}
& \frac{d X}{d \tau}=\left(X^{2}-1\right)\left(3 X \sqrt{1-Z^{2}}-\sqrt{6} \tan \left(\frac{\pi S}{2}\right)\right), \\
& \frac{d Z}{d \tau}=\left(3 X^{2}-2\right) Z \sqrt{1-Z^{2}}, \\
& \frac{d S}{d \tau}=-\frac{\sqrt{6} X(\cos (\pi S)-1)}{\pi n},
\end{aligned}
$$

defined on the compact phase space

$$
\left\{(X, Z, S) \in \mathbb{R}^{3}:-1 \leq X \leq 1,-1 \leq Z \leq 1,-1 \leq S \leq 1\right\} .
$$

The equilibrium points of (123), (124), (125) and their stability conditions are summarized as follows.

$-(X, Z, S)=(-1,-1,0)$. The eigenvalues are $\{0,-\infty, 0\}$. Nonhyperbolic.

- $(X, Z, S)=(-1,1,0)$. The eigenvalues are $\{0,-\infty, 0\}$. Nonhyperbolic.
- $(X, Z, S)=(0,-1,0)$. The eigenvalues are $\{0,0, \infty\}$. Nonhyperbolic.

$-(X, Z, S)=(0,0,0)$. The eigenvalues are $\{0,-3,-2\}$. Nonhyperbolic.

$-(X, Z, S)=(0,1,0)$. The eigenvalues are $\{0,0, \infty\}$. Nonhyperbolic.

$-(X, Z, S)=(1,-1,0)$. The eigenvalues are $\{0,-\infty, 0\}$. Nonhyperbolic.

$-(X, Z, S)=(1,1,0)$. The eigenvalues are $\{0,-\infty, 0\}$. Nonhyperbolic.

$-(X, Z, S)=\left(-\sqrt{\frac{2}{3}},-1,0\right)$. The eigenvalues are $\{0,-2,2\}$. Nonhyperbolic. Behaves as saddle.

$-(X, Z, S)=\left(\sqrt{\frac{2}{3}},-1,0\right)$. The eigenvalues are $\{0,-2$, 2\}. Nonhyperbolic. Behaves as saddle.

$-(X, Z, S)=(-1,0,0)$. The eigenvalues are $\{0,1,6\}$. Nonhyperbolic. Unstable.

- $(X, Z, S)=(1,0,0)$. The eigenvalues are $\{0,1,6\}$. Nonhyperbolic. Unstable.

$-(X, Z, S)=\left(-\sqrt{\frac{2}{3}}, 1,0\right)$. The eigenvalues are $\{0,-2$, 2\}. Nonhyperbolic. Behaves as saddle. 
$-(X, Z, S)=\left(\sqrt{\frac{2}{3}}, 1,0\right)$. The eigenvalues are $\{0,-2,2\}$.

Nonhyperbolic. Behaves as saddle.

\subsubsection{Stability analysis of the solution}

$P_{3}^{0}:(X, Z, S)=(0,0,0)$, in Hořava-Lifshitz

cosmology for the non-flat universe with $\Lambda=0$

under the detailed-balance condition the powerlawpotential

Proposition 5 The origin for the system (123), (124), (125) is unstable (saddle point).

Proof Taking the linear transformation

$\left(u, v_{1}, v_{2}\right)=\left(S, \frac{1}{6}(6 X-\sqrt{6} \pi S), Z\right)$,

and taking Taylor series near $\left(u, v_{1}, v_{2}\right)=(0,0,0)$ up to fifth order we obtain the system (123), (124), (125) can be written into its Jordan canonical form:

$$
\begin{aligned}
& \left(\begin{array}{l}
\frac{d u}{d N} \\
\frac{d v_{1}}{d N} \\
\frac{d v_{2}}{d N}
\end{array}\right)=\left(\begin{array}{ccc}
0 & 0 & 0 \\
0 & -3 & 0 \\
0 & 0 & -2
\end{array}\right)\left(\begin{array}{l}
u \\
v_{1} \\
v_{2}
\end{array}\right)+\left(\begin{array}{l}
f(u, \mathbf{v}) \\
g_{1}(u, \mathbf{v}) \\
g_{2}(u, \mathbf{v})
\end{array}\right), \\
& f(u, \mathbf{v})=-\frac{\pi u^{2}\left(\pi^{2} u^{2}-12\right)\left(\pi u+\sqrt{6} v_{1}\right)}{24 n}, \\
& g_{1}(u, \mathbf{v})=\frac{\pi^{5}(5-2 n) u^{5}}{120 \sqrt{6} n}+\frac{\pi^{4}(1-2 n) u^{4} v_{1}}{24 n} \\
& -\frac{\pi^{3} u^{3}\left(n\left(v_{1}^{2}+v_{2}^{2}-1\right)+2\right)}{4 \sqrt{6} n}-\frac{\pi^{2} u^{2} v_{1}\left(n\left(3 v_{2}^{2}-2\right)+2\right)}{4 n} \\
& +\frac{3}{8} v_{1}\left(-4\left(v_{1}^{2}-1\right) v_{2}^{2}+8 v_{1}^{2}+v_{2}^{4}\right) \\
& +\frac{1}{8} \sqrt{\frac{3}{2}} \pi u\left(v_{1}^{2}\left(16-3 v_{2}^{2}\left(v_{2}^{2}+4\right)\right)+v_{2}^{4}+4 v_{2}^{2}\right), \\
& g_{2}(u, \mathbf{v})=-\frac{1}{4} \pi^{2} u^{2}\left(v_{2}^{2}-2\right) v_{2}-\sqrt{\frac{3}{2}} \pi u v_{1}\left(v_{2}^{2}-2\right) v_{2} \\
& -\frac{3}{2} v_{1}^{2}\left(v_{2}^{2}-2\right) v_{2}+\frac{v_{2}^{5}}{4}+v_{2}^{3} .
\end{aligned}
$$

According to Theorem 1, there exists a 1-dimensional invariant local center manifold of $(127), W^{c}(\mathbf{0})=\{(u, \mathbf{v}) \in$ $\left.\mathbb{R} \times \mathbb{R}^{2}: \mathbf{v}=\mathbf{h}(u)\right\}$, satisfying $\mathbf{h}(0)=\mathbf{0}, \quad D \mathbf{h}(0)=$ $\mathbf{0},|u|<\delta$ for $\delta$ sufficiently small. The restriction of (127) to the center manifold is $\frac{d u}{d N}=f(u, \mathbf{h}(u))$, where the function h $(u)$ satisfies (A.10):

$D \mathbf{h}(u)[f(u, \mathbf{h}(u))]-P \mathbf{h}(u)-\mathbf{g}(u, \mathbf{h}(u))=0$,

where

$P=\left(\begin{array}{cc}-3 & 0 \\ 0 & -2\end{array}\right)$.

According to Theorem 3, the system (128) can be solved approximately by expanding $\mathbf{h}(u)$ in Taylor series at $u=0$. Since $\mathbf{h}(0)=\mathbf{0}$ and $D \mathbf{h}(0)=\mathbf{0}$, we propose the ansatsz $\mathbf{h}(u):=\left(\begin{array}{l}h_{1}(u) \\ h_{2}(u)\end{array}\right)=\left(\begin{array}{c}\sum_{j=1}^{4} a_{j} u^{j+1}+O\left(u^{6}\right) \\ \sum_{j=1}^{4} b_{j} u^{j+1}+O\left(u^{6}\right)\end{array}\right)$,

to find the non-null coefficients

$a_{2}=\frac{\pi^{3}(n-2)}{12 \sqrt{6} n}, \quad a_{4}=\frac{\pi^{5}(n(3 n-25)+40)}{360 \sqrt{6} n^{2}}$.

Therefore, the center manifold can be represented locally by $v_{1}=\frac{(n-2) \pi^{3} u^{3}}{12 \sqrt{6} n}+\frac{(n(3 n-25)+40) \pi^{5} u^{5}}{360 \sqrt{6} n^{2}}, \quad v_{2}=0$.

That is,

$X=\frac{\pi S}{\sqrt{6}}+\frac{\pi^{3}(n-2) S^{3}}{12 \sqrt{6} n}+\frac{\pi^{5}(n(3 n-25)+40) S^{5}}{360 \sqrt{6} n^{2}}, Z=0$

The dynamics on the center manifold is given by the gradientlike equation

$$
\begin{aligned}
\frac{d u}{d N} & =-\nabla \Pi(u) \\
\Pi(u) & =-\frac{\pi^{2} u^{4}}{8 n}+\frac{\pi^{4} u^{6}}{72 n^{2}}-\frac{\left(\pi^{6}((n-40) n+80)\right) u^{8}}{11520 n^{3}} .
\end{aligned}
$$

We have $\Pi^{\prime}(0)=\Pi^{\prime \prime}(0)=\Pi^{\prime \prime \prime(4)}(0)=-\frac{3 \pi^{2}}{n}<0$. It follows that $u=0$ is a degenerated maximum of the potential. Using the Theorem 2, we conclude that the center manifold of origin for the system (127), and the origin itself are unstable (saddle point).

\subsubsection{Alternative compactification}

In this example we can alternatively introduce the following compactification

$$
\begin{aligned}
& \bar{H}=\sqrt{H^{2}+\frac{\mu^{2} k^{2}}{16(3 \lambda-1)^{2} a^{4}}}, \\
& Q=\frac{H}{\bar{H}} \\
& \Sigma=\frac{\dot{\phi}}{2 \sqrt{6} \bar{H}}, \\
& Y=\frac{\mu \phi}{2^{\frac{1}{n}} 3^{\frac{1}{2 n}}((3 \lambda-1) n)^{\frac{1}{2 n}} \bar{H}^{\frac{1}{n}}}, \\
& T=\frac{c}{c+\bar{H}^{\frac{1}{n}}}, \quad c=2^{\frac{3}{2}-\frac{1}{n}} 3^{\frac{n-1}{2 n}} n^{-\frac{1}{2 n}}(3 \lambda-1)^{-\frac{1}{2 n}} \mu, \\
& \Sigma+Y^{2 n}=1 \text {, } \\
& \frac{d \bar{\tau}}{d t}=\bar{H}(1-T)^{-1} \text {, }
\end{aligned}
$$


such that

$$
\begin{aligned}
& \dot{\phi}=\frac{2 \sqrt{6} \Sigma c^{n}\left(\frac{1}{T}-1\right)^{n}}{Q}, \\
& \phi=\frac{2^{\frac{1}{n}} 3^{\frac{1}{2 n}} n^{\frac{1}{2 n}}\left(\frac{1}{T}-1\right) Y(3 \lambda-1)^{\frac{1}{2 n}} Q^{-1 / n} c}{\mu}, \\
& a=\frac{\sqrt{\mu} \sqrt{Q} c^{-n / 2}\left(\frac{1}{T}-1\right)^{-n / 2}}{2 \sqrt{3 \lambda-1} \sqrt[4]{1-Q^{2}}}, \\
& \bar{H}=c^{n}\left(\frac{1}{T}-1\right)^{n} .
\end{aligned}
$$

Therefore, we obtain the dynamical system

$$
\begin{aligned}
& \frac{d \Sigma}{d \bar{\tau}}=-n T Y^{2 n-1}-Q \Sigma(T-1)\left((q-1) Q^{2}-1\right), \\
& \frac{d Y}{d \bar{\tau}}=\Sigma T-\frac{Q(T-1) Y\left((q-1) Q^{2}+2\right)}{n}, \\
& \frac{d T}{d \bar{\tau}}=\frac{Q(T-1)^{2} T\left((q-1) Q^{2}+2\right)}{n}, \\
& \frac{d Q}{d \bar{\tau}}=(1-q) Q^{2}\left(Q^{2}-1\right)(T-1) .
\end{aligned}
$$

where we have the relation

$Q^{2} q=\frac{1}{2}\left(-3 Y^{2 n}+2 Q^{2}+3 \Sigma^{2}-1\right)=Q^{2}+3 \Sigma^{2}-2$.

Introducing the complementary global transformation

$\Sigma=F(\theta) \sin (\theta), \quad Y=\cos (\theta)$,

$F(\theta)=\sqrt{\frac{1-\cos ^{2 n}(\theta)}{1-\cos ^{2}(\theta)}}$,

we obtain the following unconstrained 3D dynamical system

$$
\begin{aligned}
& \frac{d \theta}{d \bar{\tau}}=-T F(\theta)+\frac{3 Q(T-1) F(\theta)^{2} \sin (2 \theta)}{2 n}, \\
& \frac{d T}{d \bar{\tau}}=-\frac{3 Q(T-1)^{2} T\left(\cos ^{2 n}(\theta)-1\right)}{n}, \\
& \frac{d Q}{d \bar{\tau}}=\left(Q^{2}-1\right)(T-1)\left(3 \cos ^{2 n}(\theta)-1\right),
\end{aligned}
$$

and the deceleration parameter satisfies

$q Q^{2}=1-3 \cos ^{2 n}(\theta)+Q^{2}$

The system (150), (151), (152) has the equilibrium points:

- $(\theta, T, Q)=\left(\frac{\pi}{2}+2 k \pi, 0, \pm 1\right) ;(\Sigma, Y)=(1,0)$, with eigenvalues $\left\{ \pm \frac{3}{n}, \pm \frac{3}{n}, \pm 2\right\}$. For $Q=+1$ it is a source. For $Q=-1$ it is a sink.
- $(\theta, T, Q)=\left(-\frac{\pi}{2}+2 k \pi, 0, \pm 1\right) ;(\Sigma, Y)=(-1,0)$, with eigenvalues $\left\{ \pm \frac{3}{n}, \pm \frac{3}{n}, \pm 2\right\}$. For $Q=+1$ it is a source. For $Q=-1$ it is a sink.

- $(\theta, T, Q)=(2 k \pi, 0, \pm 1) ;(\Sigma, Y)=(0,1)$ and $(\theta, T, Q)$ $=((2 k+1) \pi, 0, \pm 1) ;(\Sigma, Y)=(0,-1)$. The eigenvalues are $\{0, \mp 4, \mp 3\}$. For $Q=+1$ it is nonhyperbolic with a 2D stable manifold. For $Q=-1$ it is nonhyperbolic with a $2 \mathrm{D}$ unstable manifold.

- $(\theta, T, Q)=\left( \pm \arccos \left(3^{-\frac{1}{2 n}}\right), 0,0\right)$;

$(\Sigma, Y)=\left( \pm \sqrt{\frac{2}{3}}, 3^{-\frac{1}{2 n}}\right)$, with eigenvalues $0, \pm 2, \mp 2$.

These solutions corresponds to static universe $H=0$.

Nonhyperbolic. Behaves as saddle.

Substituting $T=1$ in the above equations we obtain $\frac{d \theta}{d \bar{\tau}}=$ $-F(\theta)$ that can be integrated in quadratures as

$\cos (\theta){ }_{2} F_{1}\left(\frac{1}{2}, \frac{1}{2 n} ; 1+\frac{1}{2 n} ; \cos ^{2 n}(\theta)\right)=\bar{\tau}-\bar{\tau}_{0}$.

In Fig. 5 it is shown (a) a compact phase portrait of (123), (124), (125) for the choice $n=2$. (b) Dynamics in the invariant set $Z=0$. Additionally it is presented in (c)-(f) the dynamics of the system (150), (151), (152) and some 2D projections for the choice $n=4$.

\subsubsection{Center manifold of the de Sitter solution}

$(\theta, T, Q)=(0,0,1)$ for Horava-Lifshitz with non-flat universe with $\Lambda=0$ and powerlaw potential under the detailed-balance condition

Proposition 6 The point $(\theta, T, Q)=(0,0,1)$ of the system (150), (151), (152) is unstable (saddle point).

Proof Taking the linear transformation

$\left(u, v_{1}, v_{2}\right)=\left(T, 1-Q, \frac{1}{3}(3 \theta+\sqrt{n} T)\right)$,

and truncating the Taylor series at fifth order we obtain that the system (150), (151), (152) can be written in diagonal form

$$
\left(\begin{array}{c}
\frac{d u}{d \bar{\tau}} \\
\frac{d v_{1}}{d \bar{\tau}} \\
\frac{d v_{2}}{d \bar{\tau}}
\end{array}\right)=\left(\begin{array}{ccc}
0 & 0 & 0 \\
0 & -4 & 0 \\
0 & 0 & -3
\end{array}\right)\left(\begin{array}{c}
u \\
v_{1} \\
v_{2}
\end{array}\right)+\left(\begin{array}{c}
f(u, \mathbf{v}) \\
g_{1}(u, \mathbf{v}) \\
g_{2}(u, \mathbf{v})
\end{array}\right),
$$

where

$$
\begin{aligned}
f(u, \mathbf{v})= & \frac{1}{162} n\left(-3 n^{2}+n+54\right) u^{5} \\
& +\frac{1}{3} u^{3}\left(\left(-3 n^{2}+n+9\right) v_{2}^{2}\right. \\
& \left.-12 \sqrt{n}\left(v_{1}-1\right) v_{2}-n v_{1}+n\right) \\
& +\frac{1}{27} u^{4}\left(18 n\left(v_{1}-1\right)+2 \sqrt{n}(n(3 n-1)-27) v_{2}\right) \\
& +\frac{2}{3} u^{2} v_{2}\left(3 \sqrt{n}\left(v_{1}-1\right)+\sqrt{n}(3 n-1) v_{2}^{2}\right.
\end{aligned}
$$




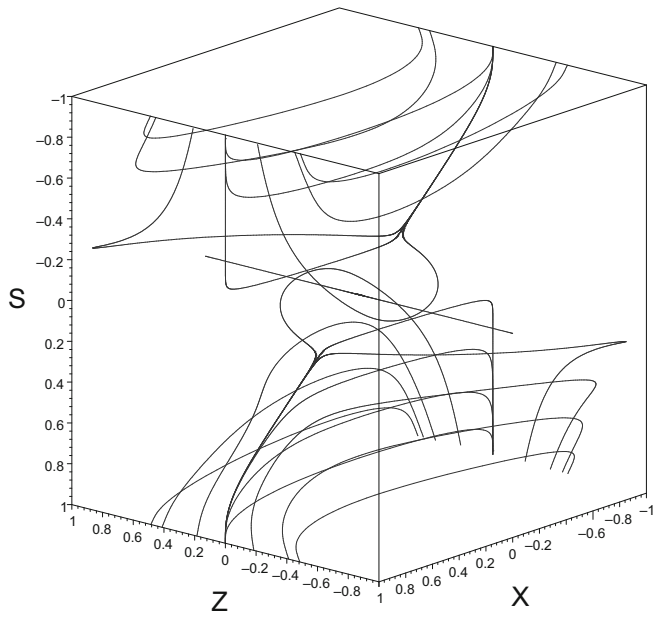

(a)

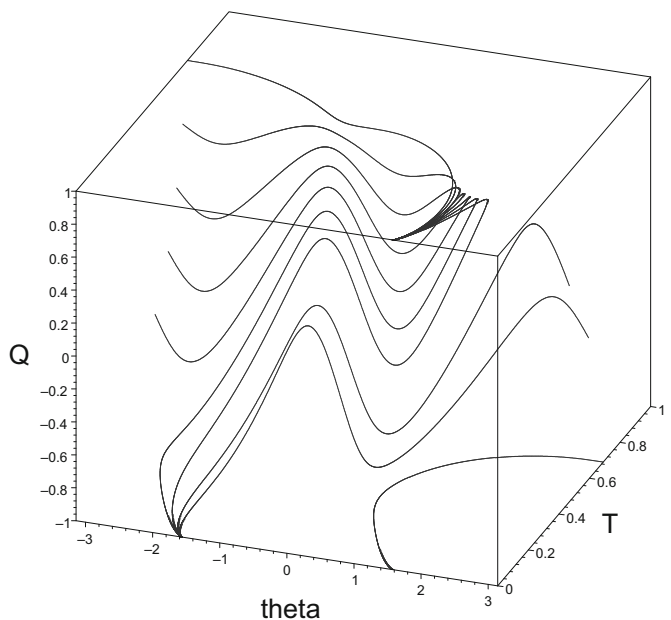

(c)

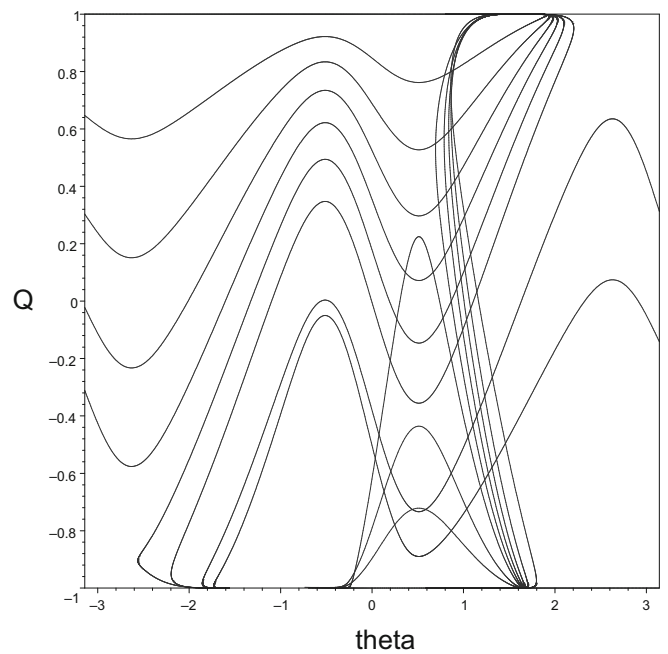

(e)

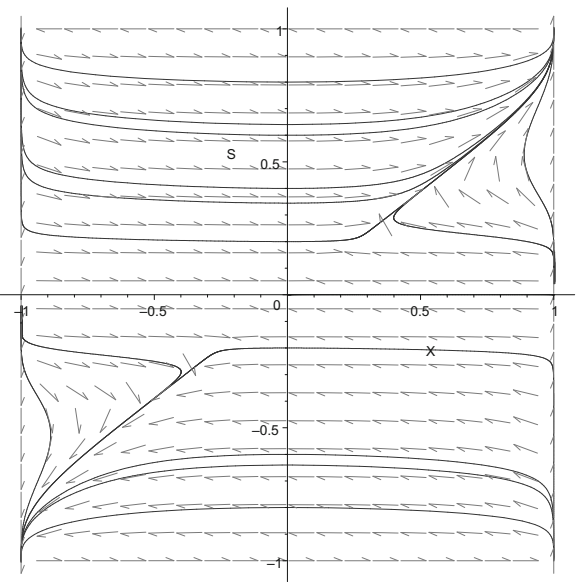

(b)

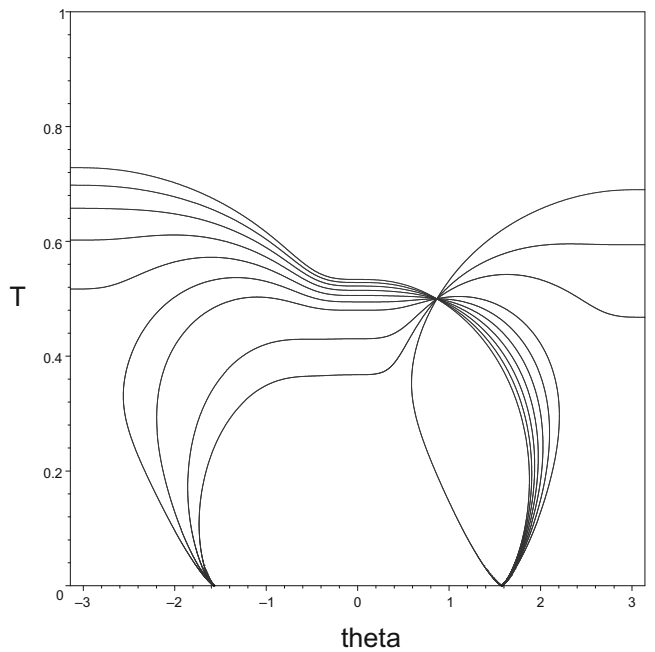

(d)

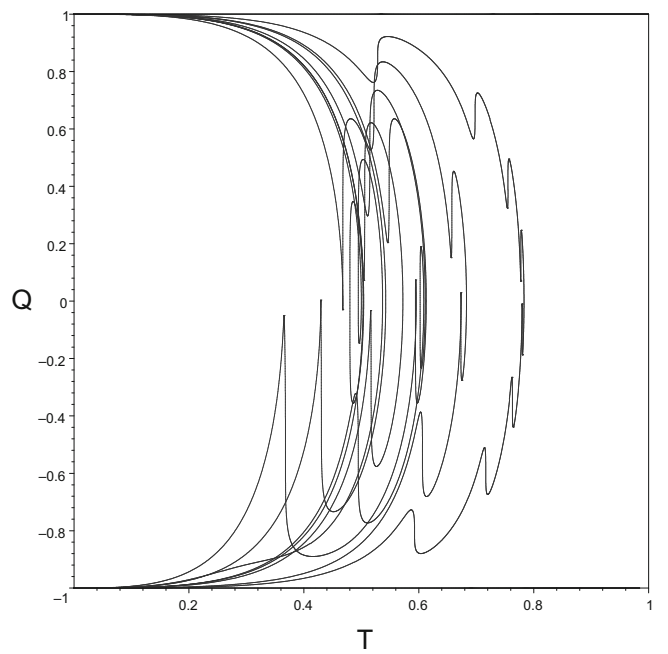

(f)

Fig. 5 a Compact phase portrait of (123), (124), (125) for the choice $n=2$. b Dynamics in the invariant set $Z=0$. c-f Dynamics of the system (150), (151), (152) and some 2D projections for the choice $n=4$ 


$$
\begin{aligned}
g_{1}(u, \mathbf{v})= & \frac{1}{3} u v_{1}\left(6 n^{3 / 2}\left(v_{1}-2\right) v_{2}+4 n^{3 / 2}(3 n-1) v_{2}^{3}\right. \\
& \left.+9 n\left(v_{1}-2\right) v_{2}^{2}-6\left(v_{1}-2\right)\right)+\frac{1}{81}(1-3 n) n^{3} u^{4} v_{1} \\
& +\frac{1}{27} u^{3}\left(4(3 n-1) n^{5 / 2} v_{1} v_{2}+9 n^{2}\left(v_{1}-2\right) v_{1}\right) \\
& -\frac{1}{3} u^{2} v_{1}\left(6 n^{3 / 2}\left(v_{1}-2\right) v_{2}+6 n^{3} v_{2}^{2}\right. \\
& \left.+n^{2}\left(v_{1}-2\left(v_{2}^{2}+1\right)\right)\right) \\
& -v_{1}\left(3 n\left(v_{1}-2\right) v_{2}^{2}+n(3 n-1) v_{2}^{4}-2 v_{1}\right), \\
g_{2}(u, \mathbf{v})= & \frac{n^{3 / 2}(n(5 n(11 n-38)+39)+4320) u^{5}}{38880} \\
& +\frac{1}{648} u^{4}\left(-12(3 n-11) n^{3 / 2}\left(v_{1}-1\right)\right. \\
& \left.-(n(3 n-7)(5 n-1)+432) n v_{2}\right) \\
& +\frac{1}{432} u^{3}\left(4 n^{3 / 2}\left(n\left(6 v_{1}-3\right)-10 v_{1}+7\right)\right. \\
& +72 n(3 n-7)\left(v_{1}-1\right) v_{2} \\
& \left.+\sqrt{n}(n(n(65 n-114)+17)+432) v_{2}^{2}\right) \\
& +\frac{1}{10}\left(\left(1-5 n^{2}\right) v_{2}^{5}-5(3 n+1)\left(v_{1}-1\right) v_{2}^{3}+30 v_{1} v_{2}\right) \\
& +u^{2}\left(-\frac{3}{2}(n-1) \sqrt{n}\left(v_{1}-1\right) v_{2}^{2}-\frac{1}{6}(n-1) n\left(3 v_{1}-2\right) v_{2}\right. \\
& \left.+\sqrt{n}\left(v_{1}-1\right)-\frac{1}{72}(n-1) n(35 n-3) v_{2}^{3}\right) \\
& +u\left(\frac{1}{2}(3 n+1)\left(v_{1}-1\right) v_{2}^{3}\right. \\
& +\frac{1}{4} \sqrt{n} v_{2}^{2}\left(n\left(6 v_{1}-5\right)-2 v_{1}+1\right) \\
& \left.-\sqrt{n} v_{1}+\frac{1}{96} \sqrt{n}(n(75 n-38)-5) v_{2}^{4}-3\left(v_{1}-1\right) v_{2}\right) .
\end{aligned}
$$

According to Theorem 1, there exists a 1-dimensional invariant local center manifold $W^{c}(\mathbf{0})$ of $(156), W^{c}(\mathbf{0})=$ $\left\{(u, \mathbf{v}) \in \mathbb{R} \times \mathbb{R}^{2}: \mathbf{v}=\mathbf{h}(u)\right\}$, satisfying $\mathbf{h}(0)=\mathbf{0}, \quad D \mathbf{h}(0)$ $=\mathbf{0},|u|<\delta$ for $\delta$ sufficiently small.

The restriction of (156) to the center manifold is $\frac{d u}{d N}=$ $f(u, \mathbf{h}(u))$, where the function $\mathbf{h}(u)$ satisfies (A.10):

$$
D \mathbf{h}(u)[f(u, \mathbf{h}(u))]-P \mathbf{h}(u)-\mathbf{g}(u, \mathbf{h}(u))=0,
$$

where

$P=\left(\begin{array}{cc}-4 & 0 \\ 0 & -3\end{array}\right)$.

According to Theorem 3, the system (157) can be solved approximately by expanding $\mathbf{h}(u)$ in Taylor series at $u=0$. Since $\mathbf{h}(0)=\mathbf{0}$ and $D \mathbf{h}(0)=\mathbf{0}$, we propose the ansatsz

$\mathbf{h}(u):=\left(\begin{array}{l}h_{1}(u) \\ h_{2}(u)\end{array}\right)=\left(\begin{array}{l}\sum_{j=1}^{4} a_{j} u^{j+1}+O\left(u^{6}\right) \\ \sum_{j=1}^{4} b_{j} u^{j+1}+O\left(u^{6}\right)\end{array}\right)$,

to find the non-null coefficients

$$
\begin{aligned}
& b_{1}=-\frac{\sqrt{n}}{3}, b_{2}=-\frac{1}{324} \sqrt{n}(n(3 n-7)+108), \\
& b_{3}=-\frac{1}{108} \sqrt{n}(n(3 n-7)+36), \\
& b_{4}=-\frac{\sqrt{n}\left(n\left(n\left(65 n^{2}-570 n+7561\right)-15120\right)+38880\right)}{116640} .
\end{aligned}
$$

Therefore, the center manifold can be represented locally by the graph

$$
\begin{aligned}
v_{1}= & 0, v_{2}=-\frac{\sqrt{n} u^{2}}{3}-\frac{1}{324}(\sqrt{n}(n(3 n-7)+108)) u^{3} \\
& -\frac{1}{108}(\sqrt{n}(n(3 n-7)+36)) u^{4} \\
& -\frac{\left(\sqrt{n}\left(n\left(n\left(65 n^{2}-570 n+7561\right)-15120\right)+38880\right)\right) u^{5}}{116640} .
\end{aligned}
$$

That is,

$$
\begin{aligned}
\theta= & -\frac{\sqrt{n} T}{3}-\frac{\sqrt{n} T^{2}}{3}-\frac{1}{324}(\sqrt{n}(n(3 n-7)+108)) T^{3} \\
& -\frac{1}{108}(\sqrt{n}(n(3 n-7)+36)) T^{4} \\
& -\frac{\left(\sqrt{n}\left(n\left(n\left(65 n^{2}-570 n+7561\right)-15120\right)+38880\right)\right) T^{5}}{116640}, \\
Q= & 1 .
\end{aligned}
$$

The dynamics on the center manifold is given by the gradientlike equation

$$
\begin{aligned}
\frac{d u}{d N} & =-\nabla \Pi(u), \Pi(u)=-\frac{n u^{4}}{12} \\
& +\frac{n^{2} u^{6}}{162}+\frac{1}{567} n^{2}(3 n+5) u^{7} \\
& +\frac{n^{2}(n(15 n(n+8)+2434)+1485) u^{8}}{174960} .
\end{aligned}
$$

We have $\Pi^{\prime}(0)=\Pi^{\prime \prime}(0)=\Pi^{\prime \prime \prime}(4)(0)=-2 n<0$. It follows that $u=0$ is a degenerated maximum of the potential. Using the Theorem 2, we conclude that the center manifold of the point $(\theta, T, Q)=(0,0,1)$ of the system (150), (151), (152), and the point itself, are unstable (saddle point).

\subsection{E-models}

In this case the system (118), (119), (120), becomes

$$
\begin{aligned}
& \frac{d X}{d \tau}=\left(X^{2}-1\right)\left(3 X \sqrt{1-Z^{2}}-\sqrt{6} \tan \left(\frac{\pi S}{2}\right)\right), \\
& \frac{d Z}{d \tau}=\left(3 X^{2}-2\right) Z \sqrt{1-Z^{2}}, \\
& \frac{d S}{d \tau}=\frac{X(-6 \mu \sin (\pi S)-\sqrt{6} \cos (\pi S)+\sqrt{6})}{\pi n},
\end{aligned}
$$


defined on the compact phase space

$\left\{(X, Z, S) \in \mathbb{R}^{3}:-1 \leq X \leq 1,-1 \leq Z \leq 1,-1 \leq S \leq 1\right\}$.

The equilibrium points of (162), (163), (164) and their stability conditions are summarized as follows:

- $(X, Z, S)=(0,-1,0)$. The eigenvalues are $\{0,0, \infty\}$. Nonhyperbolic.

$-(X, Z, S)=(0,0,0)$. The eigenvalues are $\{0,-3,-2\}$. Nonhyperbolic.

- $(X, Z, S)=(0,1,0)$. The eigenvalues are $\{0,0, \infty\}$. Nonhyperbolic.

- $(X, Z, S)=(-1,-1,0)$. The eigenvalues $\left\{0,-\infty, \frac{6 \mu}{n}\right\}$. Nonhyperbolic. Behaves as saddle.

$-(X, Z, S)=\left(-1,-1, \frac{2 \arctan (\sqrt{6} \mu)}{\pi}\right)$. The eigenvalues are $\left\{-\infty, 12 \mu,-\frac{6 \mu}{n}\right\}$. Saddle.

$-(X, Z, S)=(1,-1,0)$. The eigenvalues are $\left\{0,-\infty,-\frac{6 \mu}{n}\right\}$. Nonhyperbolic.

$-(X, Z, S)=\left(1,-1, \frac{2 \arctan (\sqrt{6} \mu)}{\pi}\right)$. The eigenvalues are $\left\{-\infty,-12 \mu, \frac{6 \mu}{n}\right\}$. Saddle.

- $(X, Z, S)=(-1,0,0)$. The eigenvalues are $\left\{1,6, \frac{6 \mu}{n}\right\}$. Source.

- $(X, Z, S)=\left(-1,0, \frac{2 \arctan (\sqrt{6} \mu)}{\pi}\right)$. The eigenvalues are $\left\{1,12 \mu+6,-\frac{6 \mu}{n}\right\}$. Saddle.

- $(X, Z, S)=(1,0,0)$. The eigenvalues are $\left\{6,1,-\frac{6 \mu}{n}\right\}$. Saddle.

$-(X, Z, S)=\left(1,0, \frac{2 \arctan (\sqrt{6} \mu)}{\pi}\right)$. The eigenvalues are $\left\{1,6-12 \mu, \frac{6 \mu}{n}\right\}$. Source for $\mu<\frac{1}{2}$.

$-(X, Z, S)=(-1,1,0)$. The eigenvalues are $\left\{0,-\infty, \frac{6 \mu}{n}\right\}$. Nonhyperbolic. Behaves as saddle.

$-(X, Z, S)=\left(-1,1, \frac{2 \arctan (\sqrt{6} \mu)}{\pi}\right)$. The eigenvalues are $\left\{-\infty, 12 \mu,-\frac{6 \mu}{n}\right\}$. Saddle.

$-(X, Z, S)=(1,1,0)$. The eigenvalues are $\left\{0,-\infty,-\frac{6 \mu}{n}\right\}$. Nonhyperbolic.

$-(X, Z, S)=\left(1,1, \frac{2 \arctan (\sqrt{6} \mu)}{\pi}\right)$. The eigenvalues are $\left\{-\infty,-12 \mu, \frac{6 \mu}{n}\right\}$. Saddle.

\subsubsection{Stability analysis of the solution} $P_{3}^{0}:(X, Z, S)=(0,0,0)$, in Horava-Lifshitz cosmology for the non-flat universe with $\Lambda=0$ under the detailed-balance condition the E-model

Proposition 7 The origin for the system (162), (163), (164) is unstable (center-saddle).

Proof Taking the linear transformation

$\left(u, v_{1}, v_{2}\right)=\left(S, \frac{1}{6}(6 X-\sqrt{6} \pi S), Z\right)$,

and taking Taylor series near $\left(u, v_{1}, v_{2}\right)=(0,0,0)$ up to fifth order we obtain the system (162), (163), (164) can be written into its Jordan canonical form:

$$
\left(\begin{array}{l}
\frac{d u}{d N} \\
\frac{d v_{1}}{d N} \\
\frac{d v_{2}}{d N}
\end{array}\right)=\left(\begin{array}{ccc}
0 & 0 & 0 \\
0 & -3 & 0 \\
0 & 0 & -2
\end{array}\right)\left(\begin{array}{c}
u \\
v_{1} \\
v_{2}
\end{array}\right)+\left(\begin{array}{c}
f(u, \mathbf{v}) \\
g_{1}(u, \mathbf{v}) \\
g_{2}(u, \mathbf{v})
\end{array}\right)
$$

where

$$
\begin{aligned}
f(u, \mathbf{v})= & -\frac{\pi^{4} u^{5}}{24 n}-\frac{\pi^{3} u^{4}\left(v_{1}-4 \mu\right)}{4 \sqrt{6} n}+\frac{\pi^{2} u^{3}\left(2 \mu v_{1}+1\right)}{2 n} \\
& +\frac{\sqrt{\frac{3}{2}} \pi u^{2}\left(v_{1}-2 \mu\right)}{n}-\frac{6 \mu u v_{1}}{n}, \\
g_{1}(u, \mathbf{v})= & \frac{\pi^{5}(5-2 n) u^{5}}{120 \sqrt{6} n}+\frac{\pi^{4} u^{4}\left(-4 \mu-2 n v_{1}+v_{1}\right)}{24 n} \\
& -\frac{\pi^{3} u^{3}\left(n\left(v_{1}^{2}+v_{2}^{2}-1\right)+4 \mu v_{1}+2\right)}{4 \sqrt{6} n} \\
& +\frac{\pi^{2} u^{2}\left(4 \mu+v_{1}\left(n\left(2-3 v_{2}^{2}\right)-2\right)\right)}{4 n} \\
& +\frac{\sqrt{\frac{3}{2}} \pi u\left(n\left(4 v_{1}^{2}\left(4-3 v_{2}^{2}\right)+v_{2}^{4}+4 v_{2}^{2}\right)+16 \mu v_{1}\right)}{8 n} \\
& +\frac{3}{8} v_{1}\left(-4\left(v_{1}^{2}-1\right) v_{2}^{2}+8 v_{1}^{2}+v_{2}^{4}\right), \\
g_{2}(u, \mathbf{v})= & -\frac{1}{4} \pi^{2} u^{2} v_{2}\left(v_{2}^{2}-2\right)-\sqrt{\frac{3}{2}} \pi u v_{1} v_{2}\left(v_{2}^{2}-2\right) \\
& +\frac{1}{4} v_{2}\left(-6 v_{1}^{2}\left(v_{2}^{2}-2\right)+v_{2}^{4}+4 v_{2}^{2}\right) .
\end{aligned}
$$

According to Theorem 1, there exists a 1-dimensional invariant local center manifold $W^{c}(\mathbf{0})$ of $(166), W^{c}(\mathbf{0})=$ $\left\{(u, \mathbf{v}) \in \mathbb{R} \times \mathbb{R}^{2}: \mathbf{v}=\mathbf{h}(u)\right\}$, satisfying $\mathbf{h}(0)=\mathbf{0}, \quad D \mathbf{h}$ $(0)=\mathbf{0},|u|<\delta$ for $\delta$ sufficiently small. The restriction of (166) to the center manifold is $\frac{d u}{d N}=f(u, \mathbf{h}(u))$, where the function $\mathbf{h}(u)$ that defines the local center manifold satisfies (A.10):

$D \mathbf{h}(u)[f(u, \mathbf{h}(u))]-P \mathbf{h}(u)-\mathbf{g}(u, \mathbf{h}(u))=0$,

where

$P=\left(\begin{array}{cc}-3 & 0 \\ 0 & -2\end{array}\right)$. 
According to Theorem 3, the system (167) can be solved approximately by expanding $\mathbf{h}(u)$ in Taylor series at $u=0$. Assuming that

$\mathbf{h}(u):=\left(\begin{array}{l}h_{1}(u) \\ h_{2}(u)\end{array}\right)=\left(\begin{array}{c}\sum_{j=1}^{4} a_{j} u^{j+1}+O\left(u^{6}\right) \\ \sum_{j=1}^{4} b_{j} u^{j+1}+O\left(u^{6}\right)\end{array}\right)$,

we find the non-null coefficients

$a_{1}=\frac{\pi^{2} \mu}{3 n}, a_{2}=\frac{\pi^{3}\left(24 \mu^{2}+(n-2) n\right)}{12 \sqrt{6} n^{2}}$,

$a_{3}=\frac{\pi^{4} \mu\left(56 \mu^{2}+n(2 n-7)\right)}{18 n^{3}}$,

$a_{4}=\frac{\pi^{5}\left(13600 \mu^{4}+n^{2}(n(3 n-25)+40)+20 \mu^{2} n(29 n-108)\right)}{360 \sqrt{6} n^{4}}$.

Therefore, the center manifold can be represented locally by the graph

$$
\begin{aligned}
& v_{1}=\frac{\pi^{2} \mu u^{2}}{3 n}+\frac{\pi^{3}\left(24 \mu^{2}+(n-2) n\right) u^{3}}{12 \sqrt{6} n^{2}} \\
& +\frac{\pi^{4} \mu\left(56 \mu^{2}+n(2 n-7)\right) u^{4}}{18 n^{3}} \\
& +\frac{\pi^{5}\left(13600 \mu^{4}+20 n(29 n-108) \mu^{2}+n^{2}(n(3 n-25)+40)\right) u^{5}}{360 \sqrt{6} n^{4}} \\
& v_{2}=0 .
\end{aligned}
$$

That is,

$$
\begin{aligned}
& X=\frac{\pi S}{\sqrt{6}}+\frac{\pi^{2} \mu S^{2}}{3 n}+\frac{\pi^{3}\left(24 \mu^{2}+(n-2) n\right) S^{3}}{12 \sqrt{6} n^{2}} \\
& +\frac{\pi^{4} \mu\left(56 \mu^{2}+n(2 n-7)\right) S^{4}}{18 n^{3}} \\
& +\frac{\pi^{5}\left(13600 \mu^{4}+20 n(29 n-108) \mu^{2}+n^{2}(n(3 n-25)+40)\right) S^{5}}{360 \sqrt{6} n^{4}}, \\
& Z=0 .
\end{aligned}
$$

The dynamics on the center manifold is given by the gradientlike equation

$$
\begin{aligned}
\frac{d u}{d N} & =-\nabla \Pi(u), \\
\Pi(u) & =\frac{\sqrt{\frac{2}{3}} \pi \mu u^{3}}{n}-\frac{\pi^{2} u^{4}\left(n-4 \mu^{2}\right)}{8 n^{2}} \\
& -\frac{\pi^{3} \mu u^{5}\left(n(n+4)-24 \mu^{2}\right)}{10 \sqrt{6} n^{3}} \\
& +\frac{\pi^{4} u^{6}\left(224 \mu^{4}+n^{2}+4 \mu^{2}(n-10) n\right)}{72 n^{4}} \\
& -\frac{\pi^{5} \mu u^{7}\left(-6800 \mu^{4}+n^{2}(n(n+15)-55)+10 \mu^{2}(136-23 n) n\right)}{210 \sqrt{6} n^{5}} .
\end{aligned}
$$

We have $\Pi^{\prime}(0)=\Pi^{\prime \prime}(0)=0, \Pi^{\prime \prime \prime}(0)=\frac{2 \sqrt{6} \pi \mu}{n}$. It follows that $u=0$ is an inflection of the potential. Using the Theorem 2, we conclude that the center manifold of origin for the system (166), and the origin are unstable (center-saddle).

\subsubsection{Alternative compactification}

In this example we can alternatively introduce the following compactification

$$
\begin{aligned}
\bar{H} & =\sqrt{H^{2}+\frac{\mu^{2} k^{2}}{16(3 \lambda-1)^{2} a^{4}}}, \\
Q & =\frac{H}{\bar{H}}, \quad \Sigma=\frac{\dot{\phi}}{2 \sqrt{6} \bar{H}}, \\
Y & =\left(\frac{V(\phi)}{6(3 \lambda-1) \bar{H}^{2}}\right)^{\frac{1}{2 n}}=\bar{T}\left(1-e^{-\sqrt{\frac{2}{3 \alpha}} \phi}\right), \\
\bar{T} & =\left[\frac{V_{0}}{6(3 \lambda-1) \bar{H}^{2}}\right]^{\frac{1}{2 n}},
\end{aligned}
$$

such that

$$
\begin{aligned}
& \dot{\phi}=\frac{\sqrt{\frac{2}{3}} \Sigma V_{0} \bar{T}^{-2 n}}{3 \lambda-1}, \\
& \phi=-\frac{\sqrt{\frac{2}{3}} n \ln \left(1-\frac{Y}{T}\right)}{\mu}, \\
& H=Q \sqrt{\frac{V_{0}}{6(3 \lambda-1)}} \bar{T}^{-n}, \\
& a=\frac{\sqrt[4]{3} \sqrt{\mu} \bar{T}^{n / 2}}{2^{3 / 4} \sqrt[4]{3 \lambda-1} \sqrt[4]{1-Q^{2}} \sqrt[4]{V_{0}}},
\end{aligned}
$$

and

$\Sigma+Y^{2 n}=1$

$Q^{2} q=\frac{1}{2}\left(-3 Y^{2 n}+2 Q^{2}+3 \Sigma^{2}-1\right)=Q^{2}+3 \Sigma^{2}-2$.

Introducing the new time variable $\frac{d M}{d t}=\frac{H}{Q}$, the dynamical system becomes

$$
\begin{aligned}
& \frac{d \Sigma}{d M}=6 \mu Y^{2 n-1}(Y-\bar{T})+Q \Sigma\left((q-1) Q^{2}-1\right) \\
& \frac{d Y}{d M}=\frac{(q-1) Q^{3} Y+2 Q Y+6 \mu \Sigma(\bar{T}-Y)}{n} \\
& \frac{d \bar{T}}{d M}=\frac{Q \bar{T}\left((q-1) Q^{2}+2\right)}{n} \\
& \frac{d Q}{d M}=(q-1) Q^{2}\left(Q^{2}-1\right)
\end{aligned}
$$

and the deceleration parameter satisfies

$q Q^{2}=1-3 \cos ^{2 n}(\theta)+Q^{2}$. 
Introducing the complementary global transformation

$\Sigma=F(\theta) \sin (\theta), \quad Y=\cos (\theta)$,

$F(\theta)=\sqrt{\frac{1-\cos ^{2 n}(\theta)}{1-\cos ^{2}(\theta)},}$

we obtain the following regular unconstrained 3D dynamical system

$\frac{d \theta}{d M}=-\frac{6 \mu F(\theta)(\bar{T}-\cos (\theta))}{n}-\frac{3 Q F(\theta)^{2} \sin (2 \theta)}{2 n}$,

$\frac{d \bar{T}}{d M}=-\frac{3 Q \bar{T}\left(\cos ^{2 n}(\theta)-1\right)}{n}$,

$\frac{d Q}{d M}=\left(1-Q^{2}\right)\left(3 \cos ^{2 n}(\theta)-1\right)$.

Introducing the compact variable $T=\frac{\bar{T}}{1+\bar{T}}$ and the time derivative $\frac{d \bar{\tau}}{d M}=1+\bar{T}=(1-T)^{-1}$, we obtain the regular system:

$$
\begin{aligned}
\frac{d \theta}{d \bar{\tau}}= & -\frac{6 \mu F(\theta)((T-1) \cos (\theta)+T)}{n} \\
& +\frac{3 Q(T-1) F(\theta)^{2} \sin (2 \theta)}{2 n}, \\
\frac{d T}{d \bar{\tau}}= & -\frac{3 Q(T-1)^{2} T\left(\cos ^{2 n}(\theta)-1\right)}{n}, \\
\frac{d Q}{d \bar{\tau}}= & \left(Q^{2}-1\right)(T-1)\left(3 \cos ^{2 n}(\theta)-1\right) .
\end{aligned}
$$

The past boundary is attached to the phase-space, and it is $\{T=0, \cos (\theta) \leq 0\} \cup\{T-(1-T) \cos \theta=0, \cos (\theta)>0\}$. It is also included the future boundary $T=1$, which corresponds to $H=0$ and the final state is the Minkowski point. The region $\{T-(1-T) \cos \theta<0, \cos (\theta)>0\}$ is forbidden. We include the boundaries $Q= \pm 1$ too. The equilibrium points of (191), (192), (193) and their stability is summarized as follows.

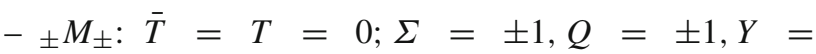
$0 ; \theta= \pm \frac{\pi}{2}+2 k \pi, k \in \mathbb{Z}$, were we have used the Kernel: $\operatorname{sign}(Q) M_{\operatorname{sign}(\Sigma)}$. The eigenvalues of ${ }_{-} M_{+}$are $\left\{-\frac{3}{n},-2,-\frac{6 \mu+3}{n}\right\}$. It is a sink. The eigenvalues of $-M_{-}$ are $\left\{-\frac{3}{n},-2,-\frac{3-6 \mu}{n}\right\}$. It is a sink for $\mu<\frac{1}{2}$ or a saddle otherwise. The eigenvalues of $+M_{+}$are $\left\{\frac{3}{n}, 2, \frac{3-6 \mu}{n}\right\}$. It is a source for $\mu<\frac{1}{2}$ or a saddle otherwise. The eigenvalues of ${ }_{+} M_{-}$are $\left\{\frac{3}{n}, 2, \frac{6 \mu+3}{n}\right\}$. It is a source.

- $d S^{ \pm}: \bar{T}=1, T=\frac{1}{2} ; \Sigma=0, Q= \pm 1, Y=1 ; \theta=$ $2 k \pi, k \in \mathbb{Z}$. The eigenvalues of $d S^{+}$are $\left\{0,-2,-\frac{3}{2}\right\}$. Nonhyperbolic with 2D stable manifold. The eigenvalues of $d S^{-}$are $\left\{0,2, \frac{3}{2}\right\}$. Nonhyperbolic with 2D unstable manifold.

- $P L^{ \pm}: \bar{T}=T=0 ; \Sigma= \pm 2 \mu, Q= \pm 1 ; Y=$ $-\left(1-4 \mu^{2}\right)^{\frac{1}{2 n}} ; \theta= \pm \arccos Y$. Exists for $\mu<1 / 2$. The eigenvalues are $\left\{ \pm \frac{12 \mu^{2}}{n}, \pm\left(24 \mu^{2}-4\right), \pm\left(12 \mu^{2}-3\right)\right\}$. It is a saddle.

- $L_{1}^{ \pm}: \bar{T}=T=0 ; \Sigma= \pm \sqrt{\frac{2}{3}}, Q= \pm \sqrt{6} \mu$; $Y=3^{-\frac{1}{2 n}}, \theta= \pm \arccos \left(3^{-\frac{1}{2 n}}\right)$. The eigenvalues are $\left\{ \pm \frac{2 \sqrt{6} \mu}{n}, \mp \frac{\sqrt{3} \mu+\sqrt{8-45 \mu^{2}}}{\sqrt{2}}, \mp \frac{\sqrt{3} \mu-\sqrt{8-45 \mu^{2}}}{\sqrt{2}}\right\}$. Saddle. - $S^{ \pm}: \bar{T}=3^{-\frac{1}{2 n}}, T=\frac{1}{1+3^{\frac{1}{2 n}}} ; \Sigma= \pm \sqrt{\frac{2}{3}}, Q=$ $0 ; Y=3^{-\frac{1}{2 n}} ; \theta= \pm \arccos \left(3^{-\frac{1}{2 n}}\right)$. The eigenvalues are $\left\{ \pm 2 \frac{3 \frac{1}{2 n}}{3^{\frac{1}{2 n}}+1}, \mp 2 \frac{3 \frac{1}{2 n}}{3^{\frac{1}{2 n}}+1}, \mp \frac{2 \sqrt{6} \mu}{n} \frac{3 \frac{1}{2 n}}{3^{\frac{1}{2 n}}+1}\right\}$. It is a saddle.

Substituting $T=1$ in the above equations we obtain $\frac{d \theta}{d \bar{\tau}}=$ $-\frac{6 \mu}{n} F(\theta)$. This equation can be integrated in quadratures as $\cos (\theta){ }_{2} F_{1}\left(\frac{1}{2}, \frac{1}{2 n} ; 1+\frac{1}{2 n} ; \cos ^{2 n}(\theta)\right)=\frac{6 \mu}{n}\left(\bar{\tau}-\bar{\tau}_{0}\right)$.

In Fig. 6 it is shown (a) a compact phase portrait of (162), (163), (164) for the choice $n=2, \mu=1$. (b) Dynamics in the invariant set $Z=0$. In (c)-(f) it is presented the dynamics of (191), (192), (193) and some 2D projections for $n=4, \mu=$ 1.

\subsubsection{Center manifold of the de Sitter solution} $(\theta, T, Q)=\left(0, \frac{1}{2}, 1\right)$ for Hor̆ava-Lifshitz with non-flat universe with $\Lambda=0$ and E-potential under the detailed-balance condition

Proposition 8 The point $(\theta, T, Q)=\left(0, \frac{1}{2}, 1\right)$ of the system (191), (192), (193) is unstable (center-saddle).

Proof Taking the linear transformation

$\left(u, v_{1}, v_{2}\right)=\left(\frac{1}{2}(2 T-1), 1-Q, \frac{-4 \mu+\theta \sqrt{n}+8 \mu T}{\sqrt{n}}\right)$,

and taking Taylor series near $\left(u, v_{1}, v_{2}\right)=(0,0,0)$ up to fifth order we obtain the system (191), (192), (193) can be written into its Jordan canonical form:

$$
\left(\begin{array}{l}
\frac{d u}{d N} \\
\frac{d v_{1}}{d N} \\
\frac{d v_{2}}{d N}
\end{array}\right)=\left(\begin{array}{ccc}
0 & 0 & 0 \\
0 & -2 & 0 \\
0 & 0 & -\frac{3}{2}
\end{array}\right)\left(\begin{array}{c}
u \\
v_{1} \\
v_{2}
\end{array}\right)+\left(\begin{array}{c}
f(u, \mathbf{v}) \\
g_{1}(u, \mathbf{v}) \\
g_{2}(u, \mathbf{v})
\end{array}\right),
$$

where 


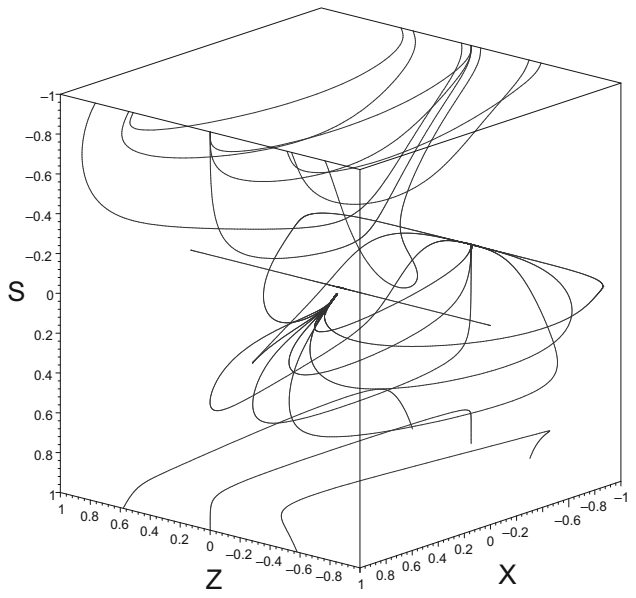

(a)

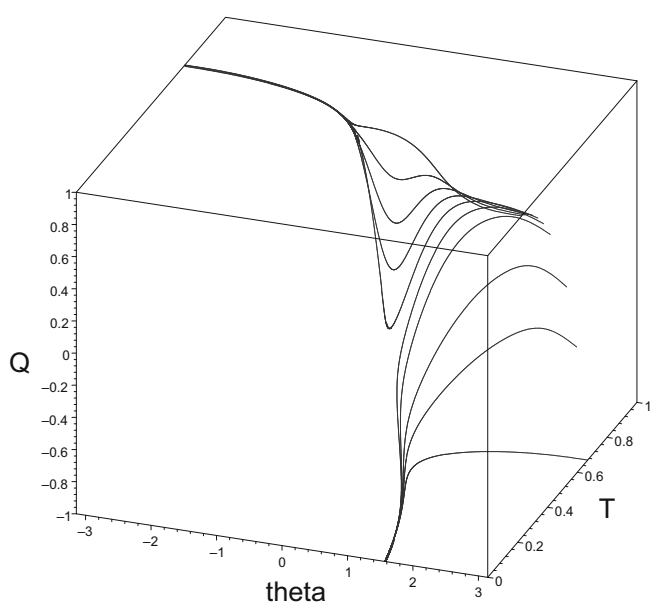

(c)

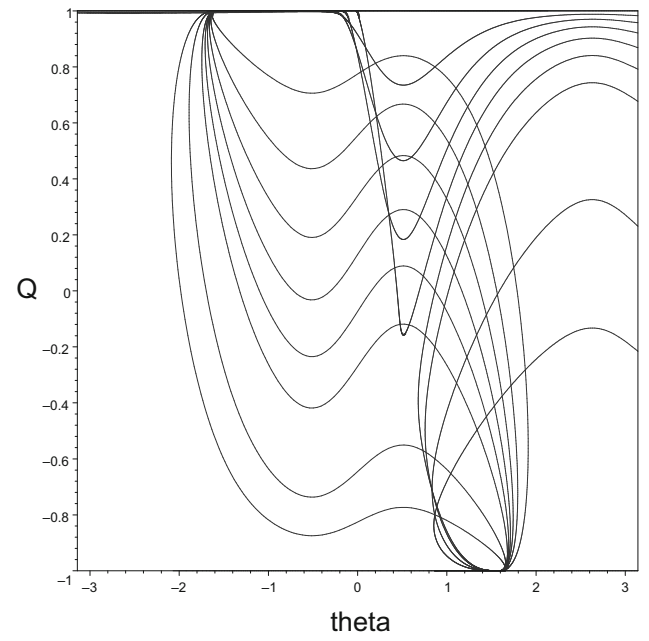

(e)

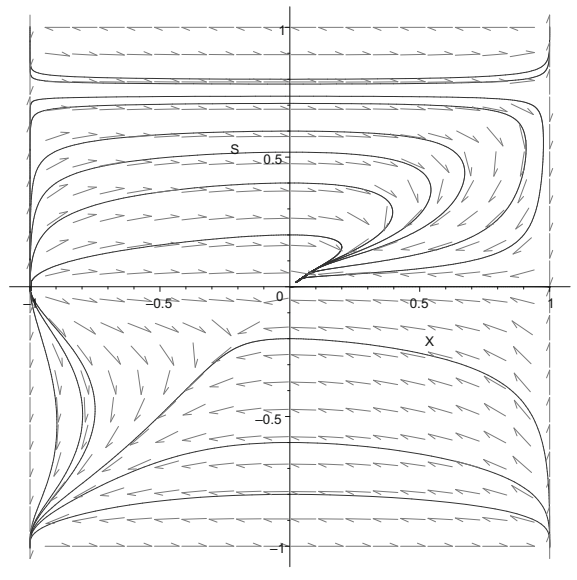

(b)

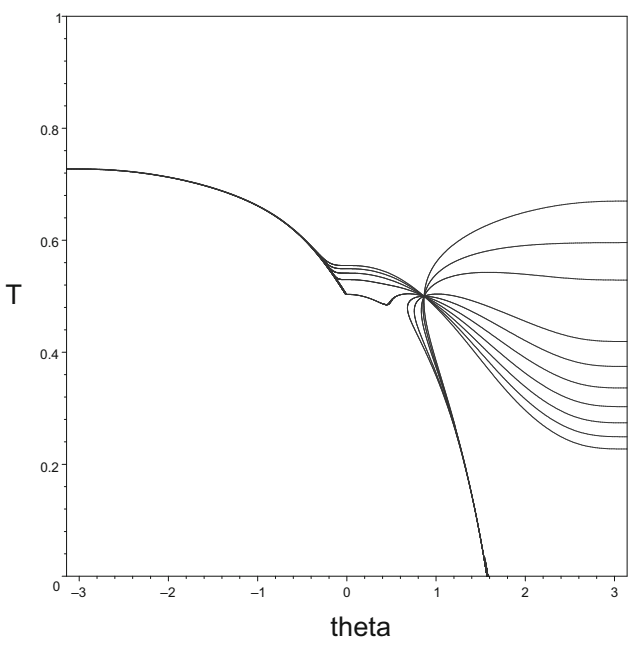

(d)

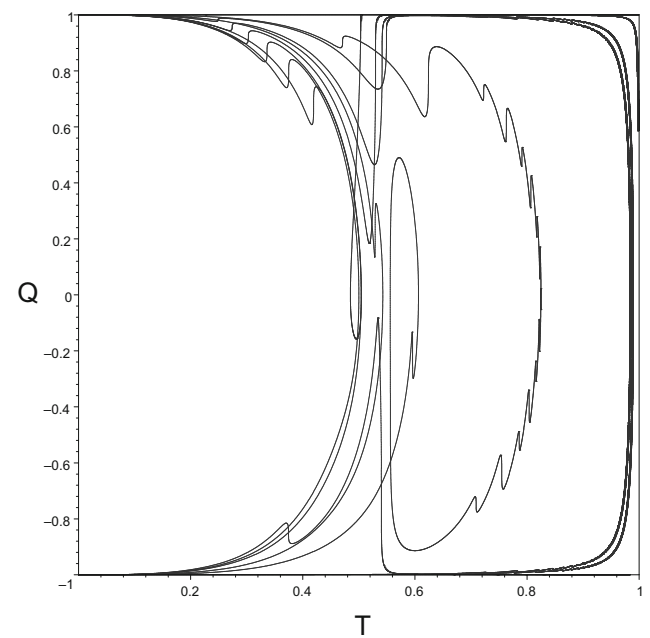

(f)

Fig. 6 a Compact phase portrait of (162), (163), (164) for the choice $n=2, \mu=1$. b Dynamics in the invariant set $Z=0$. c-f Dynamics of the system (191), (192), (193) and some 2D projections for $n=4, \mu=1$ 


$$
\begin{aligned}
& f(u, \mathbf{v})=\frac{u^{3}\left(-3 n^{3 / 2}\left(v_{1}-1\right) v_{2}^{2}+48 \mu^{2} \sqrt{n}\left((3 n-1) v_{2}^{2}+v_{1}-1\right)-128 \mu^{3}(3 n-1)\left(v_{1}-1\right) v_{2}-24 \mu n\left(v_{1}-1\right) v_{2}\right)}{n^{3 / 2}} \\
& -\frac{64 \mu^{2} u^{5}\left(8 \mu^{2}+3 n\left(-8 \mu^{2}+v_{1}-1\right)\right)}{n^{2}}+\frac{16 \mu u^{4}\left(-3 n^{3 / 2} v_{2}+16 \mu^{3}(3 n-1)\left(v_{1}-1\right)+6 \mu n\left(v_{1}-1\right)+16 \mu^{2}(1-3 n) \sqrt{n} v_{2}\right)}{n^{2}} \\
& +\frac{u^{2}\left(48 \mu^{2}\left(v_{1}-1\right)\left((3 n-1) v_{2}^{2}-1\right)+8 \mu \sqrt{n} v_{2}\left((1-3 n) v_{2}^{2}-3 v_{1}+3\right)+3 n\left(v_{1}-1\right) v_{2}^{2}\right)}{2 n} \\
& +\frac{u v_{2}\left(\sqrt{n} v_{2}\left(v_{2}^{2}\left(3 n+v_{1}-1\right)+6\left(v_{1}-1\right)\right)-16 \mu\left(v_{1}-1\right)\left((3 n-1) v_{2}^{2}-3\right)\right)}{8 \sqrt{n}}+\frac{1}{16}\left(v_{1}-1\right) v_{2}^{2}\left((3 n-1) v_{2}^{2}-6\right), \\
& g_{1}(u, \mathbf{v})=\frac{2048(1-3 n) u^{4} v_{1} \mu^{4}}{n}+64 u^{3} v_{1}\left(3 v_{1}+\frac{16(3 n-1) v_{2} \mu}{\sqrt{n}}-6\right) \mu^{2} \\
& -48 u^{2} v_{1}\left(\sqrt{n}\left(v_{1}-2\right) v_{2}+2\left((6 n-2) v_{2}^{2}+v_{1}-2\right) \mu\right) \mu+\frac{1}{2} v_{1}\left((1-3 n) n v_{2}^{4}-3 n\left(v_{1}-2\right) v_{2}^{2}+2 v_{1}\right) \\
& +u v_{1}\left(3 n^{2} v_{2}^{4}-n v_{2}^{4}-6 n v_{2}^{2}+3 n v_{1} v_{2}^{2}+8 \sqrt{n}\left((6 n-2) v_{2}^{2}+3 v_{1}-6\right) \mu v_{2}-2 v_{1}+4\right) \\
& g_{2}(u, \mathbf{v})=\frac{512 \mu^{3}\left((5 n(11 n+28)-61) \mu^{2}-15 n\left(v_{1}-1\right)\right) u^{5}}{5 n^{5 / 2}}+\frac{128 \mu^{2}\left(4\left(-4 v_{1}+3 n\left(4 v_{1}-3\right)+2\right) \mu^{3}-2 \sqrt{n}(n(15 n+28)-13) v_{2} \mu^{2}-2 n(3 n-2)\left(v_{1}-1\right) \mu-3 n^{3 / 2} v_{2}\right) u^{4}}{n^{5 / 2}} \\
& +\frac{8 \mu\left(-32\left(-4 v_{1}+3 n\left(4 v_{1}-3\right)+2\right) v_{2} \mu^{3}+2 \sqrt{n}\left((n(65 n+84)-43) v_{2}^{2}+4\left(6 v_{1} n-3 n+8 v_{1}-8\right)\right) \mu^{2}+12 n(3 n-1)\left(v_{1}-1\right) v_{2} \mu+3 n^{3 / 2} v_{2}^{2}\right) u^{3}}{n^{2}} \\
& +\frac{4 \mu\left(12\left(\left(-4 v_{1}+3 n\left(4 v_{1}-3\right)+2\right) v_{2}^{2}-4 v_{1}+2\right) \mu^{2}+\sqrt{n} v_{2}\left((17-7 n(5 n+4)) v_{2}^{2}+12\left(2 n-3(n+1) v_{1}+3\right)\right) \mu-3 n\left(v_{1}-1\right)\left(3 n v_{2}^{2}-2\right)\right) u^{2}}{n^{3 / 2}} \\
& +\frac{\left(-160 v_{2}\left(\left(-4 v_{1}+3 n\left(4 v_{1}-3\right)+2\right) v_{2}^{2}-12 v_{1}+6\right) \mu^{2}+5 \sqrt{n}\left((n(75 n+28)-25) v_{2}^{4}+24\left(6 v_{1} n-5 n+4 v_{1}-4\right) v_{2}^{2}-96 v_{1}\right) \mu+4 n v_{2}\left(-v_{2}^{4}+5(3 n+1)\left(v_{1}-1\right) v_{2}^{2}-30\left(v_{1}-1\right)\right)\right) u}{40 n} \\
& +\frac{v_{2}\left(2 \sqrt{n}\left(\left(1-5 n^{2}\right) v_{2}^{4}-5(3 n+1)\left(v_{1}-1\right) v_{2}^{2}+30 v_{1}\right)+5 v_{2}\left(\left(-4 v_{1}+3 n\left(4 v_{1}-3\right)+2\right) v_{2}^{2}-24 v_{1}+12\right) \mu\right)}{40 \sqrt{n}} .
\end{aligned}
$$

According to Theorem 1, there exists a 1-dimensional invariant local center manifold $W^{c}(\mathbf{0})$ of $(196), W^{c}(\mathbf{0})=$ $\left\{(u, \mathbf{v}) \in \mathbb{R} \times \mathbb{R}^{2}: \mathbf{v}=\mathbf{h}(u)\right\}$, satisfying $\mathbf{h}(0)=\mathbf{0}, \quad D \mathbf{h}(0)$ $=\mathbf{0},|u|<\delta$ for $\delta$ sufficiently small. The restriction of (196) to the center manifold is $\frac{d u}{d N}=f(u, \mathbf{h}(u))$, where the function $\mathbf{h}(u)$ satisfies (A.10):

$$
D \mathbf{h}(u)[f(u, \mathbf{h}(u))]-P \mathbf{h}(u)-\mathbf{g}(u, \mathbf{h}(u))=0,
$$

where

$$
P=\left(\begin{array}{cc}
-2 & 0 \\
0 & -\frac{3}{2}
\end{array}\right) \text {. }
$$

According to Theorem 3, the system (197) can be solved approximately by expanding $\mathbf{h}(u)$ in Taylor series at $u=0$. Since $\mathbf{h}(0)=\mathbf{0}$ and $D \mathbf{h}(0)=\mathbf{0}$, we propose the ansatsz

$\mathbf{h}(u):=\left(\begin{array}{l}h_{1}(u) \\ h_{2}(u)\end{array}\right)=\left(\begin{array}{l}\sum_{j=1}^{4} a_{j} u^{j+1}+O\left(u^{6}\right) \\ \sum_{j=1}^{4} b_{j} u^{j+1}+O\left(u^{6}\right)\end{array}\right)$,

Comparing the coefficients of the equal powers of $u$ we find the non-null coefficients

$$
\begin{aligned}
& b_{1}=-\frac{16 \mu\left(n-4 \mu^{2}\right)}{n^{3 / 2}}, \quad b_{2}=-\frac{32\left(288 \mu^{5}+3 \mu n^{2}+4 \mu^{3} n(3 n-13)\right)}{3 n^{5 / 2}}, \\
& b_{3}=\frac{64 \mu\left(3648 \mu^{6}-n^{3}+4 \mu^{2}(10-3 n) n^{2}+48 \mu^{4} n(3 n-16)\right)}{n^{7 / 2}}, \\
& b_{4}=\frac{128 \mu\left(-2711040 \mu^{8}-15 n^{4}-360 \mu^{2}(n-3) n^{3}-8 \mu^{4} n^{2}(5 n(13 n-330)+5761)-960 \mu^{6} n(121 n-687)\right)}{15 n^{9 / 2}} .
\end{aligned}
$$


Therefore, the center manifold can be represented locally by the graph

$$
\begin{aligned}
v_{1} & =0, v_{2}=-\frac{16\left(\mu\left(n-4 \mu^{2}\right)\right) u^{2}}{n^{3 / 2}}-\frac{32\left(288 \mu^{5}+4 n(3 n-13) \mu^{3}+3 n^{2} \mu\right) u^{3}}{3 n^{5 / 2}} \\
& +\frac{64 \mu\left(3648 \mu^{6}+48 n(3 n-16) \mu^{4}+4(10-3 n) n^{2} \mu^{2}-n^{3}\right) u^{4}}{n^{7 / 2}} \\
& +\frac{128 \mu\left(-2711040 \mu^{8}-960 n(121 n-687) \mu^{6}-8 n^{2}(5 n(13 n-330)+5761) \mu^{4}-360(n-3) n^{3} \mu^{2}-15 n^{4}\right) u^{5}}{15 n^{9 / 2}} .
\end{aligned}
$$

That is

$$
\begin{aligned}
& \theta=-\frac{4 \mu(2 T-1)}{\sqrt{n}}-\frac{4 \mu(2 T-1)^{2}\left(n-4 \mu^{2}\right)}{n^{3 / 2}}-\frac{4(2 T-1)^{3}\left(288 \mu^{5}+3 \mu n^{2}+4 \mu^{3} n(3 n-13)\right)}{3 n^{5 / 2}} \\
& +\frac{4 \mu(2 T-1)^{4}\left(3648 \mu^{6}-n^{3}+4 \mu^{2}(10-3 n) n^{2}+48 \mu^{4} n(3 n-16)\right)}{n^{7 / 2}} \\
& +\frac{4 \mu(2 T-1)^{5}\left(-2711040 \mu^{8}-15 n^{4}-360 \mu^{2}(n-3) n^{3}-8 \mu^{4} n^{2}(5 n(13 n-330)+5761)-960 \mu^{6} n(121 n-687)\right)}{15 n^{9 / 2}}, \\
& Q=1 .
\end{aligned}
$$

The dynamics on the center manifold is given by the gradient-like equation

$$
\begin{aligned}
\frac{d u}{d N} & =-\nabla \Pi(u), \\
\Pi(u) & =-\frac{8 \mu^{2} u^{3}}{n}-\frac{1536 \mu^{4} u^{5}\left(13 \mu^{2}-2 n\right)}{5 n^{3}}-\frac{12 \mu^{2} u^{4}\left(n-8 \mu^{2}\right)}{n^{2}} \\
& +\frac{512 u^{6}\left(504 \mu^{8}+4 \mu^{4} n^{2}+\mu^{6} n(12 n-101)\right)}{n^{4}} \\
& -\frac{6144 \mu^{4} u^{7}\left(24992 \mu^{6}-6 n^{3}+\mu^{2}(385-72 n) n^{2}+8 \mu^{4} n(106 n-745)\right)}{7 n^{5}} .
\end{aligned}
$$

We have $\Pi^{\prime}(0)=\Pi^{\prime \prime}(0)=0, \Pi^{\prime \prime \prime}(0)=-\frac{48 \mu^{2}}{n}<0$. It follows that $u=0$ is an inflection of the potential. Using the Theorem 2, we conclude that the center manifold of origin for the system (196), and the origin are unstable (center-saddle).

\section{Case 3: Flat universe with $\Lambda \neq 0$ under the detailed-balance condition}

The field equations of HL for a Flat universe with $\Lambda \neq 0$ under the detailed-balance condition are:

$$
\begin{aligned}
H^{2} & =\frac{1}{6(3 \lambda-1)}\left[\frac{3 \lambda-1}{4} \dot{\phi}^{2}+V(\phi)\right] \\
& -\frac{\mu^{2} \Lambda^{2}}{16(3 \lambda-1)^{2}}, \\
\dot{H} & +\frac{3}{2} H^{2}=-\frac{1}{4(3 \lambda-1)}\left[\frac{3 \lambda-1}{4} \dot{\phi}^{2}-V(\phi)\right]
\end{aligned}
$$

$$
\begin{gathered}
-\frac{3 \mu^{2} \Lambda^{2}}{32(3 \lambda-1)^{2}}, \\
\ddot{\phi}+3 H \dot{\phi}+\frac{2 V^{\prime}(\phi)}{3 \lambda-1}=0 .
\end{gathered}
$$

\subsection{Arbitrary potential}

In this case the system (202), (203), (204) is given in its autonomous form:

$$
\begin{aligned}
& \frac{d x}{d N}=\sqrt{6} s\left(u^{2}-x^{2}+1\right)+3 x\left(x^{2}-1\right) \\
& \frac{d u}{d N}=3 u x^{2} \\
& \frac{d s}{d N}=-2 \sqrt{6} x f(s) .
\end{aligned}
$$

defined on the phase space $\left\{(x, u, s) \in \mathbb{R}^{3}: x^{2}-u^{2} \leq 1\right\}$.

The equilibrium points/curves at the finite region of the phase space of (205), (206), (207) are presented in Table 5, where is shown the existence and stability conditions. We proceed to the discussion of the more relevant features of them.

- $P_{9}(\hat{s}):(x, u, s)=(1,0, \hat{s})$, where $f(\hat{s})=0$. It is a source for $f^{\prime}(\hat{s})<0, \hat{s}<\sqrt{\frac{3}{2}}$.

- $P_{10}(\hat{s}):(x, u, s)=(-1,0, \hat{s})$, where $f(\hat{s})=0$. It is a source for $f^{\prime}(\hat{s})>0, \hat{s}>-\sqrt{\frac{3}{2}}$. 
Table 5 Case 3: Equilibrium points at the finite region of the system (205), (206), (207)

\begin{tabular}{|c|c|c|c|c|}
\hline Equil. points & $(x, u, s)$ & Existence & Eigenvalues & Stability \\
\hline$P_{9}(\hat{s})$ & $(1,0, \hat{s})$ & $f(\hat{s})=0$ & $6-2 \sqrt{6} \hat{s}, 3,-2 \sqrt{6} f^{\prime}(\hat{s})$ & $\begin{array}{l}\text { Nonhyperbolic for } \\
\qquad f^{\prime}(\hat{s})=0, \text { or } \hat{s}=\sqrt{\frac{3}{2}} \\
\text { Source for } \\
\quad f^{\prime}(\hat{s})<0, \hat{s}<\sqrt{\frac{3}{2}} \\
\text { Saddle otherwise }\end{array}$ \\
\hline$P_{10}(\hat{s})$ & $(-1,0, \hat{s})$ & $f(\hat{s})=0$ & $6+2 \sqrt{6} \hat{s}, 3,2 \sqrt{6} f^{\prime}(\hat{s})$ & $\begin{array}{l}\text { Nonhyperbolic for } \\
\qquad f^{\prime}(\hat{s})=0, \text { or } \hat{s}=-\sqrt{\frac{3}{2}} \\
\text { Source for } \\
\quad f^{\prime}(\hat{s})>0, \hat{s}>-\sqrt{\frac{3}{2}} \\
\text { Saddle otherwise }\end{array}$ \\
\hline$P_{11}(\hat{s})$ & $\left(\sqrt{\frac{2}{3}} \hat{s}, 0, \hat{s}\right)$ & $\begin{array}{l}f(\hat{s})=0 \\
-\sqrt{\frac{3}{2}} \leq \hat{s} \leq \\
\sqrt{\frac{3}{2}}\end{array}$ & $2 \hat{s}^{2}, 2 \hat{s}^{2}-3,-4 \hat{s} f^{\prime}(\hat{s})$ & $\begin{array}{l}\text { Nonhyperbolic for } \\
\quad f^{\prime}(\hat{s})=0 \text {, or } \\
\hat{s} \in\left\{-\sqrt{\frac{3}{2}}, 0, \sqrt{\frac{3}{2}}\right\} \\
\text { Saddle otherwise }\end{array}$ \\
\hline$P_{11}^{0}\left(u_{c}\right):$ & $\left(0, u_{c}, 0\right)$ & $u_{c} \in \mathbb{R}$ & $0,-\frac{3}{2} \pm \frac{1}{2} \sqrt{9-48 f(0)\left(u_{c}^{2}+1\right)}$ & Nonhyperbolic \\
\hline
\end{tabular}

Table 6 Case 3: Equilibrium points at the infinity region of the system (205), (206), (207)

\begin{tabular}{lllll}
\hline Equil. points & $(x, U, S)$ & Existence & Eigenvalues & Stability \\
\hline$Q_{19,20}(\hat{s})$ & $\left(-1, \pm 1, \frac{2}{\pi} \arctan (\hat{s})\right)$ & $f(\hat{s})=0$ & $-\infty, 2 \sqrt{6} \hat{s}, 2 \sqrt{6} f^{\prime}(\hat{s})$ & Sinks for $\hat{s}<0, f^{\prime}(\hat{s})<0$ \\
$Q_{21,22}(\hat{s})$ & $\left(1, \pm 1, \frac{2}{\pi} \arctan (\hat{s})\right)$ & $f(\hat{s})=0$ & $-\infty,-2 \sqrt{6} \hat{s},-2 \sqrt{6} f^{\prime}(\hat{s})$ & Sinks for $\hat{s}>0, f^{\prime}(\hat{s})>0$ \\
$Q_{23,24}$ & $(0, \pm 1,0)$ & Always & $0,-2 i \sqrt{3} \sqrt{f(0)}, 2 i \sqrt{3} \sqrt{f(0)}$ & Saddle for $f(0)<0$. \\
& & & Center for $f(0)>0$ \\
\hline
\end{tabular}

$-P_{11}(\hat{s}):(x, u, s)=\left(\sqrt{\frac{2}{3}} \hat{s}, 0, \hat{s}\right)$, where $f(\hat{s})=0$,

$\frac{d X}{d \tau}=\left(X^{2}-1\right)\left(3 \sqrt{1-U^{2}} X-\sqrt{6} \tan \left(\frac{\pi S}{2}\right)\right)$,

$-\sqrt{\frac{3}{2}} \leq \hat{s} \leq \sqrt{\frac{3}{2}}$. It is a saddle.

- $P_{11}^{0}\left(u_{c}\right):(x, u, s)=\left(0, u_{c}, 0\right)$. This line of equilibrium points is new, and it was not found in [41]. It is nonhyperbolic.

- There are two lines of equilibrium points $P_{12,13}\left(s_{c}\right)$ : $(x, u, s)=\left(0, \pm i, s_{c}\right), s_{c} \in \mathbb{R}$ which are not considered since they are complex valued.

Due to the dynamical system (205), (206), (207) is unbounded, we introduce the new variables

$X=\frac{x}{\sqrt{1+u^{2}}}, \quad U=\frac{u}{\sqrt{1+u^{2}}}, \quad S=\frac{2}{\pi} \arctan (s)$,

and the time rescaling $\frac{d f}{d \tau}=\sqrt{1-U^{2}} \frac{d f}{d N}$ to obtain the dynamical system

$\frac{d U}{d \tau}=3 U \sqrt{1-U^{2}} X^{2}$,

$\frac{d S}{d \tau}=-\frac{2 \sqrt{6} X(\cos (\pi S)+1) f\left(\tan \left(\frac{\pi S}{2}\right)\right)}{\pi}$,

defined on the compacted phase space

$\left\{(X, U, S) \in \mathbb{R}^{3}:-1 \leq X \leq 1,-1 \leq U \leq 1,-1 \leq S \leq 1\right\}$.

The points at the infinite region of the phase space are summarized in Table 6 . Now we discuss the relevant features of them.

$-Q_{19,20}(\hat{s}):(X, U, S)=\left(-1, \pm 1, \frac{2}{\pi} \arctan (\hat{s})\right)$. It is a sink for $\hat{s}<0, f^{\prime}(\hat{s})<0$

- $Q_{21,22}(\hat{s}):(X, U, S)=\left(1, \pm 1, \frac{2}{\pi} \arctan (\hat{s})\right)$, where $f(\hat{s})=0$. It is a sink for $\hat{s}>0, f^{\prime}(\hat{s})>0$.

- $Q_{23,24}:(X, U, S)=(0, \pm 1,0)$. Always exists. It is a Saddle for $f(0)<0$. It is a Center for $f(0)>0$. 
Fig. 7 Compact phase portrait of (212), (213) for different choices of the parameter $s$
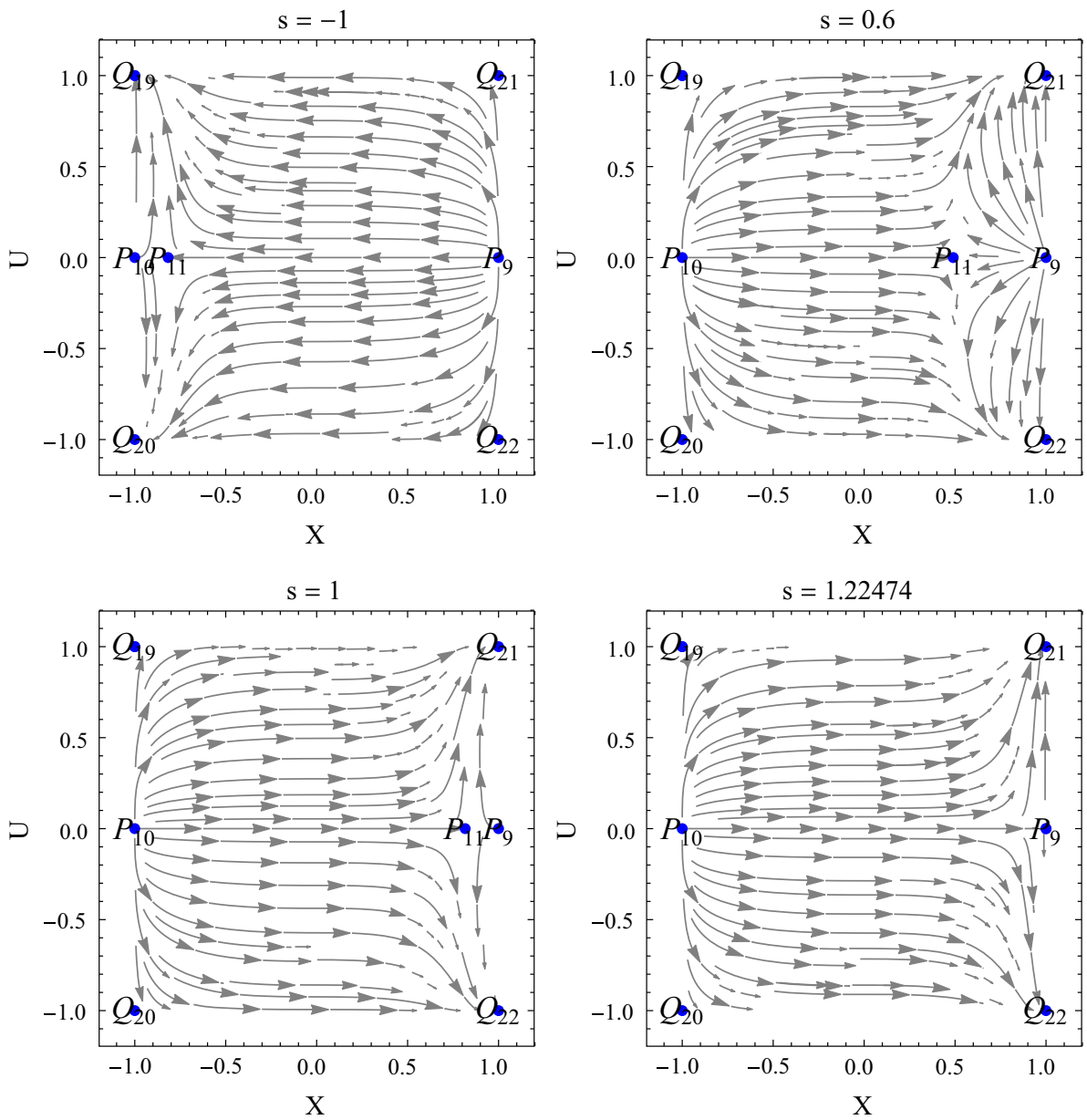

\subsection{Exponential potential}

In this example the system (209), (210) becomes

$$
\begin{aligned}
& \frac{d X}{d \tau}=\left(X^{2}-1\right)\left(3 X \sqrt{1-U^{2}}-\sqrt{6} s\right), \\
& \frac{d U}{d \tau}=3 X^{2} U \sqrt{1-U^{2}}
\end{aligned}
$$

defined on the compact phase space

$\left\{(X, U) \in \mathbb{R}^{2}:-1 \leq X \leq 1,-1 \leq U \leq 1\right\}$.

Under this scenario, the Hořava-Lifshitz universe admits two unstable equilibrium points $\left(P_{9,10}\right)$, completely dominated by stiff dark matter.

Point $P_{11}$ exhibits a more physical dark matter equationof-state parameter, but still with negligible dark energy at late times.

Figure 7a illustrates when $Q_{19,20}:(X, U)=(-1, \pm 1)$ is a sink for $s<0$. Figure $7 \mathrm{~b}-\mathrm{d}$ illustrates when $Q_{21,22}$ : $(X, U)=(1, \pm 1)$ is a sink for $s>0$.

\subsection{Powerlaw potential}

In this example the system (209), (210), (211) becomes

$$
\begin{aligned}
\frac{d X}{d \tau} & =\left(X^{2}-1\right)\left(3 \sqrt{1-U^{2}} X-\sqrt{6} \tan \left(\frac{\pi S}{2}\right)\right), \\
\frac{d U}{d \tau} & =3 U \sqrt{1-U^{2}} X^{2}, \\
\frac{d S}{d \tau} & =-\frac{\sqrt{6} X(\cos (\pi S)-1)}{\pi n},
\end{aligned}
$$

defined on the compact phase space

$$
\left\{(X, U, S) \in \mathbb{R}^{3}:-1 \leq X \leq 1,-1 \leq U \leq 1,-1 \leq S \leq 1\right\} .
$$

The coordinates equilibrium points of (214), (215), (216) and their stability conditions are summarized as follows:

$-(x, U, S)=\left(0, U_{c}, 0\right)$ with eigenvalues $\{0,0$, $\left.-3 \sqrt{1-U_{c}^{2}}\right\}$. Non-hyperbolic with a 1 dimensional stable manifold. 
- $(x, U, S)=(-1,0,0)$ with eigenvalues $\{6,3,0\}$.

Non-hyperbolic with a 2 dimensional unstable manifold.

- $(x, U, S)=(1,0,0)$ with eigenvalues $\{6,3,0\}$.

Non-hyperbolic with a 2 dimensional unstable manifold.

$-(x, U, S)=(-1,-1,0)$ with eigenvalues $\{0,-\infty, 0\}$. Non-hyperbolic with a 1 dimensional stable manifold.

$-(x, U, S)=(-1,1,0)$ with eigenvalues $\{0,-\infty, 0\}$. Non-hyperbolic with a 1 dimensional stable manifold.

$-(x, U, S)=(1,-1,0)$ with eigenvalues $\{0,-\infty, 0\}$. Non-hyperbolic with a 1 dimensional stable manifold.

- $(x, U, S)=(1,1,0)$ with eigenvalues $\{0,-\infty, 0\}$. Nonhyperbolic with a 1 dimensional stable manifold.

\subsubsection{Alternative compactification}

Introducing the following compactification

$$
\begin{aligned}
\hat{H} & =\sqrt{H^{2}+\frac{\mu^{2} \Lambda^{2}}{16(3 \lambda-1)^{2}}}, \\
Q & =\frac{H}{\hat{H}}, \\
\Sigma & =\frac{\dot{\phi}}{2 \sqrt{6} \hat{H}}, \\
Y & =\frac{\mu \phi}{2^{\frac{1}{n}} 3^{\frac{1}{2 n}}((3 \lambda-1) n)^{\frac{1}{2 n}} \hat{H}^{\frac{1}{n}}}, \\
T & =\frac{c}{c+\hat{H}^{\frac{1}{n}}}, \quad c=2^{\frac{3}{2}-\frac{1}{n} 3^{\frac{n-1}{2 n}}} n^{-\frac{1}{2 n}}(3 \lambda-1)^{-\frac{1}{2 n}} \mu, \\
\Sigma & +Y^{2 n}=1 .
\end{aligned}
$$

such that

$$
\begin{aligned}
& \dot{\phi}=2 \sqrt{6} \Sigma c^{n}\left(\frac{1}{T}-1\right)^{n}, \\
& \phi=\frac{2^{\frac{1}{n}} 3 \frac{1}{2 n} n^{\frac{1}{2 n}}\left(\frac{1}{T}-1\right) Y(3 \lambda-1)^{\frac{1}{2 n}} c}{\mu}, \\
& \Lambda=\frac{4(3 \lambda-1) \sqrt{1-Q^{2}} c^{n}\left(\frac{1}{T}-1\right)^{n}}{\mu}, \\
& \hat{H}=c^{n}\left(\frac{1}{T}-1\right)^{n}
\end{aligned}
$$

and the new time variable $\frac{d \hat{\tau}}{d t}=\hat{H}(1-T)^{-1}$, we obtain the dynamical system

$$
\begin{aligned}
& \frac{d \Sigma}{d \hat{\tau}}=-n T Y^{2 n-1}-Q \Sigma(T-1)\left((q+1) Q^{2}-3\right), \\
& \frac{d Y}{d \hat{\tau}}=\Sigma T-\frac{(q+1) Q^{3}(T-1) Y}{n}, \\
& \frac{d T}{d \hat{\tau}}=\frac{(q+1) Q^{3}(T-1)^{2} T}{n},
\end{aligned}
$$

$\frac{d Q}{d \hat{\tau}}=-(q+1) Q^{2}\left(Q^{2}-1\right)(T-1)$

where

$Q^{2} q=\frac{1}{2}\left(-3 Y^{2 n}-2 Q^{2}+3 \Sigma^{2}+3\right)=3 \Sigma^{2}-Q^{2}$.

Introducing the complementary global transformation

$\Sigma=F(\theta) \sin (\theta), \quad Y=\cos (\theta)$,

$F(\theta)=\sqrt{\frac{1-\cos ^{2 n}(\theta)}{1-\cos ^{2}(\theta)}}$,

we obtain the following unconstrained 3D dynamical system

$$
\begin{aligned}
& \frac{d \theta}{d \hat{\tau}}=\frac{3 Q(T-1) F(\theta)^{2} \sin (2 \theta)}{2 n}-T F(\theta), \\
& \frac{d T}{d \hat{\tau}}=\frac{3 Q(T-1)^{2} T\left(1-\cos ^{2 n}(\theta)\right)}{n}, \\
& \frac{d Q}{d \hat{\tau}}=-3\left(Q^{2}-1\right)(T-1)\left(1-\cos ^{2 n}(\theta)\right) .
\end{aligned}
$$

where

$Q^{2} q=-3 \cos ^{2 n}(\theta)-Q^{2}+3$.

The equilibrium points of (233), (234), (235) and their stability conditions are summarized as follows:

${ }_{-} M_{+}:(\theta, T, Q)=\left(\frac{\pi}{2}+2 k \pi, 0, \pm 1\right) .(\Sigma, Y)=(1,0)$. The eigenvalues are $\left\{ \pm 6, \pm \frac{3}{n}, \pm \frac{3}{n}\right\} .-M_{+}$is a sink whereas $+M_{+}$is a source.

${ }_{-}{ }_{ \pm} M_{-}:(\theta, T, Q)=\left(-\frac{\pi}{2}+2 k \pi, 0, \pm 1\right) .(\Sigma, Y)=$ $(-1,0)$. In the last two cases we used the Kernel: $\operatorname{sign}(Q) M_{\operatorname{sign}(\Sigma)}$. The eigenvalues are $\left\{ \pm 6, \pm \frac{3}{n}, \pm \frac{3}{n}\right\}$. $-M_{-}$is a sink whereas $+M_{-}$is a source.

$-d S^{ \pm}:(\theta, T, Q)=(2 k \pi, 0, \pm 1) ;(\Sigma, Y)=(0,1)$ and $d S_{-}^{ \pm}:(\theta, T, Q)=((2 k+1) \pi, 0, \pm 1) ;(\Sigma, Y)=$ $(0,-1)$. The eigenvalues are $\mp 3,0,0$. Nonhyperbolic, 2 dimensional center manifold. In general we have the line $d S_{+}\left(Q^{*}\right):(\theta, T, Q)=\left(2 k \pi, 0, Q^{*}\right) ;(\Sigma, Y)=$ $(0,1)$ and $d S_{-}\left(Q^{*}\right):(\theta, T, Q)=\left((2 k+1) \pi, 0, Q^{*}\right)$; $(\Sigma, Y)=(0,-1)$. The eigenvalues are $-3 Q^{*}, 0,0$. Nonhyperbolic, 2 dimensional center manifold.

Substituting $T=1$ in the above equations we obtain $\frac{d \theta}{d \hat{\tau}}=$ $-F(\theta)$. This equation can be integrated in quadratures as

$$
\cos (\theta)_{2} F_{1}\left(\frac{1}{2}, \frac{1}{2 n} ; 1+\frac{1}{2 n} ; \cos ^{2 n}(\theta)\right)=\left(\hat{\tau}-\hat{\tau}_{0}\right) .
$$

In the Fig. 8 it is shown (a) a compact phase portrait of the system (214), (215), (216) for the choice $n=2$. (b) Dynamics in the invariant set $U=0$. In Fig. 8c-f it is presented 


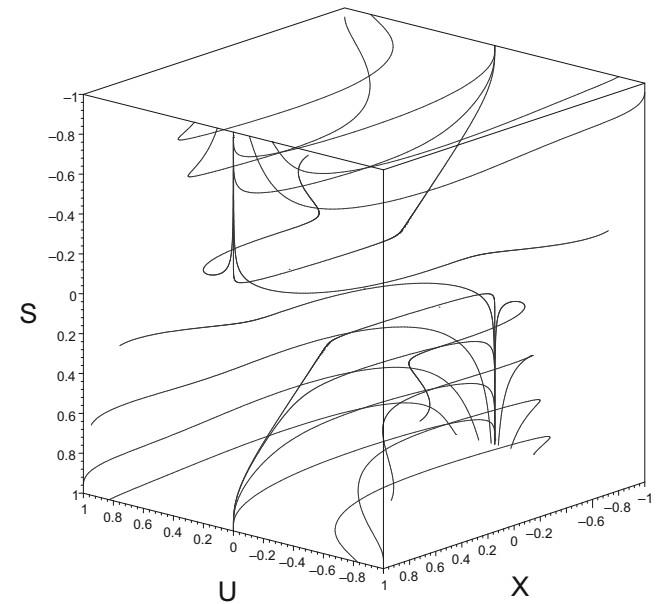

(a)

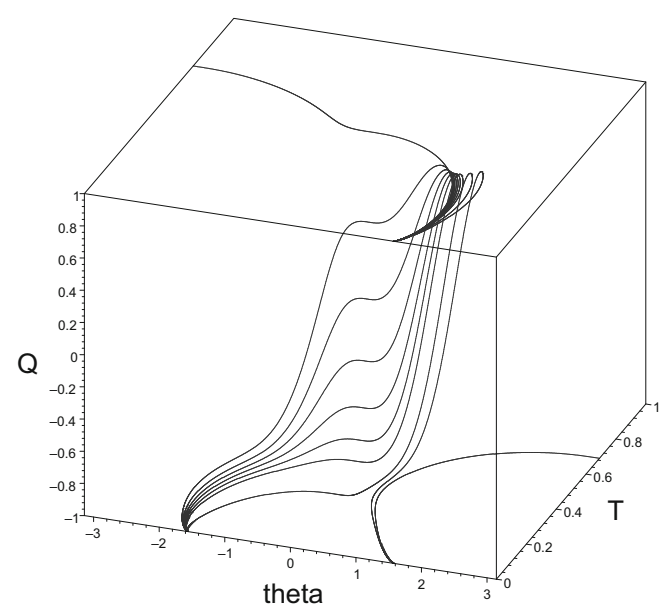

(c)

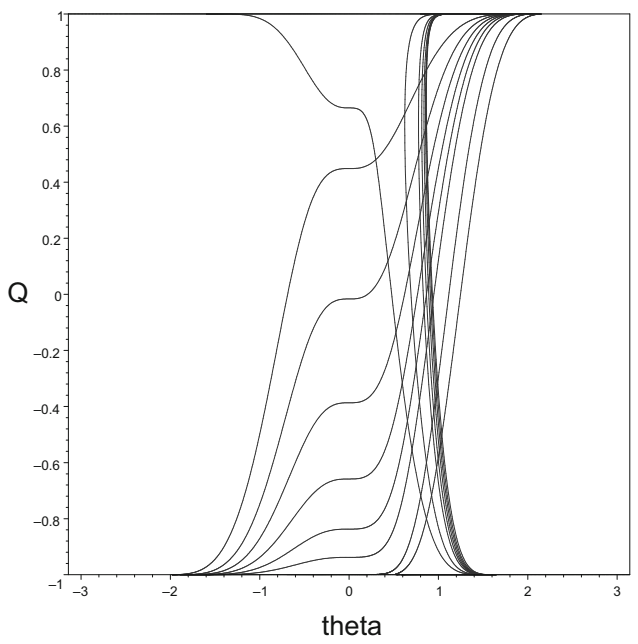

(e)

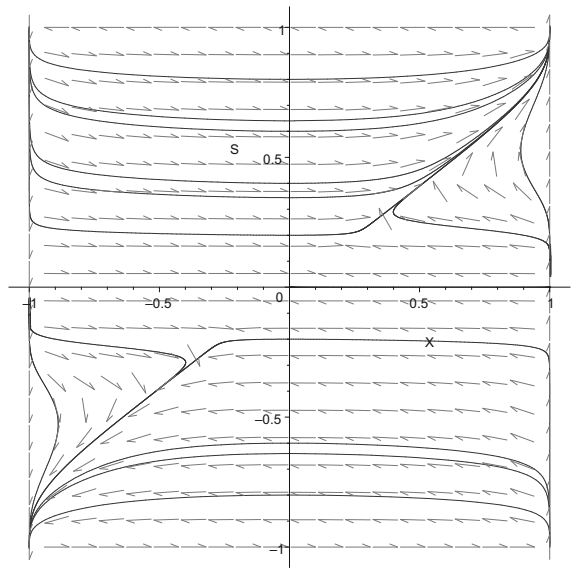

(b)

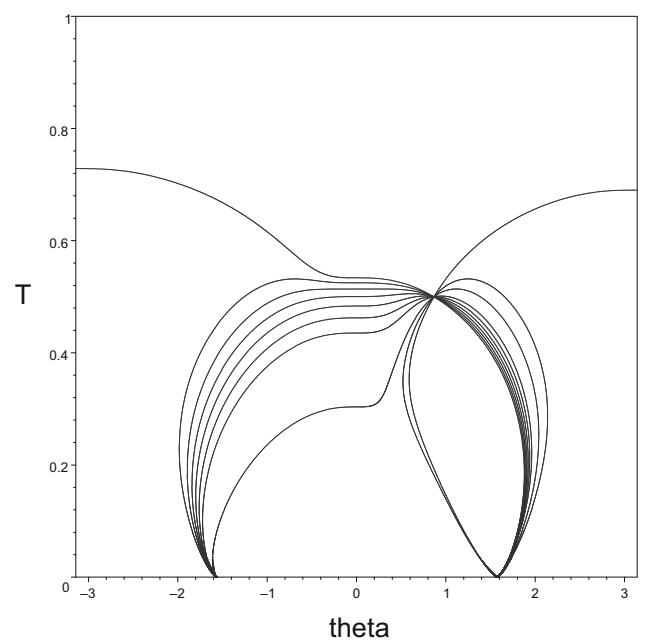

(d)

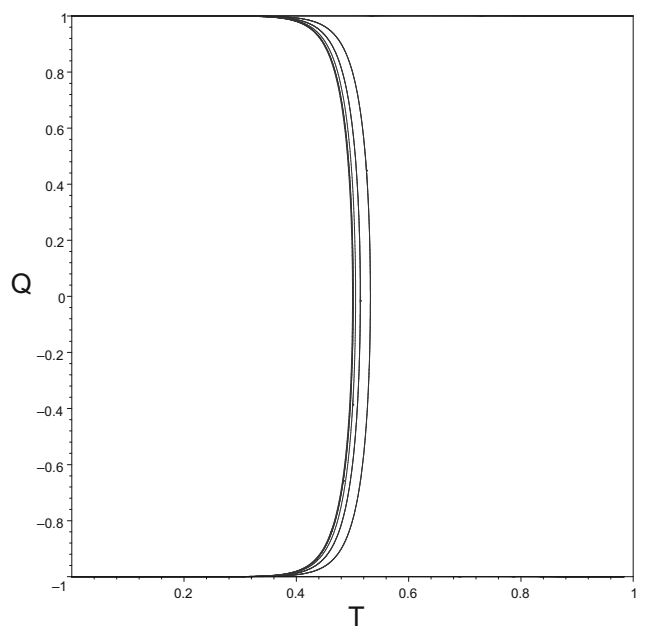

(f)

Fig. 8 a Compact phase portrait of (214), (215), (216) for the choice $n=2$. b Dynamics in the invariant set $U=0$. c-f Dynamics of (233), (234), (235), and some 2D projections of the solutions for $n=4, \mu=1$ 
the dynamics of the system (233), (234), (235) and some 2D projections of the solutions for $n=4, \mu=1$.

\subsubsection{Center manifold of the de Sitter solution for: (i) $d S^{+}:(\theta, T, Q)=(0,0,1),($ ii $)$ $d S^{+}\left(Q^{*}\right):(\theta, T, Q)=\left(0,0, Q^{*}\right), Q^{*} \neq \pm 1,0$, for Hořava-Lifshitz with flat universe with $\Lambda \neq 0$ and powerlaw potential under the detailed-balance}

Proposition 9 (i) The point $d S^{+}:(\theta, T, Q)=(0,0,1)$ of the system (233), (234), (235) is unstable (saddle point). (ii) The line of fixed points $d S_{+}\left(Q^{*}\right):(\theta, T, Q)=\left(0,0, Q^{*}\right)$, $Q^{*} \neq \pm 1,0$ of the system (233), (234), (235) is unstable (saddle).

Proof Part (i) Taking the linear transformation

$$
\begin{aligned}
& \left(u_{1}, u_{2}, v\right)=\left(T, 1-Q, \frac{1}{3}(3 \theta+\sqrt{n} T)\right), \\
& u_{1} \in[0,1], u_{2} \in[0,2],
\end{aligned}
$$

and taking Taylor series near $\left(u_{1}, u_{2}, v\right)=(0,0,0)$ up to fifth order we obtain the system (233), (234), (235) can be written into its Jordan canonical form:

$$
\left(\begin{array}{c}
\frac{d u_{1}}{d N} \\
\frac{d u_{2}}{d N} \\
\frac{d v}{d N}
\end{array}\right)=\left(\begin{array}{ccc}
0 & 0 & 0 \\
0 & 0 & 0 \\
0 & 0 & -3
\end{array}\right)\left(\begin{array}{c}
u_{1} \\
u_{2} \\
v
\end{array}\right)+\left(\begin{array}{c}
f_{1}(\mathbf{u}, v) \\
f_{2}(\mathbf{u}, v) \\
g(\mathbf{u}, v)
\end{array}\right),
$$

where

$$
\begin{aligned}
& f_{1}(\mathbf{u}, v)=\frac{1}{162} n\left(-3 n^{2}+n+54\right) u_{1}^{5} \\
&+u_{1}^{3}\left(\frac{1}{3}\left(\left(-3 n^{2}+n+9\right) v^{2}+12 \sqrt{n} v+n\right)\right. \\
&\left.+u_{2}\left(-4 \sqrt{n} v-\frac{n}{3}\right)\right) \\
&+u_{1}^{4}\left(\frac{2}{3} u_{2}(3 \sqrt{n} v+n)+\frac{2}{27}(n(3 n-1)-27) \sqrt{n} v-\frac{2 n}{3}\right) \\
&+u_{1}^{2}\left(2 u_{2} v(\sqrt{n}+3 v)+\frac{2}{3} v\left(\sqrt{n}\left((3 n-1) v^{2}-3\right)-9 v\right)\right) \\
&+u_{1}\left(\frac{1}{2}(1-3 n) v^{4}-3 u_{2} v^{2}+3 v^{2}\right), \\
& f_{2}(\mathbf{u}, v)=u_{1}\left(u_{2}\left(4 n^{5 / 2} v^{3}-\frac{4}{3} n^{3 / 2}\left(v^{2}+3\right) v-6 n v^{2}\right)\right. \\
&\left.+n u_{2}^{2} v(2 \sqrt{n}+3 v)\right) \\
&+\frac{1}{81}(1-3 n) n^{3} u_{1}^{4} u_{2}+u_{1}^{3} \\
&+\left(\frac{n^{2} u_{2}^{2}}{3}+\frac{2}{27} n^{2} u_{2}(2 \sqrt{n}(3 n-1) v-9)\right) \\
&+u_{1}^{2}\left(u_{2}^{2}\left(-2 n^{3 / 2} v-\frac{n^{2}}{3}\right)\right. \\
&\left.+u_{2}\left(4 n^{3 / 2} v-2 n^{3} v^{2}+\frac{2}{3} n^{2}\left(v^{2}+1\right)\right)\right) \\
&-3 n u_{2}^{2} v^{2}+n u_{2} v^{2}\left((1-3 n) v^{2}+6\right),
\end{aligned}
$$

$$
\begin{aligned}
g(\mathbf{u}, v)= & \frac{n^{3 / 2}(n(5 n(11 n-38)+39)+4320) u_{1}^{5}}{38880} \\
& +u_{1}^{4}\left(\frac{1}{54}(11-3 n) n^{3 / 2} u_{2}+\frac{1}{54}(3 n-11) n^{3 / 2}\right. \\
& \left.-\frac{1}{648}(n(3 n-7)(5 n-1)+432) n v\right) \\
& +u_{1}^{3}\left(\frac{1}{54} u_{2}\left((3 n-5) n^{3 / 2}+9(3 n-7) n v\right)\right. \\
& +\frac{1}{432}\left(4(7-3 n) n^{3 / 2}+\sqrt{n}(n(n(65 n-114)\right. \\
& \left.\left.+17)+432) v^{2}+72(7-3 n) n v\right)\right) \\
& +u_{1}^{2}\left(\frac{1}{2} \sqrt{n} u_{2}(2-(n-1) v(\sqrt{n}+3 v))\right. \\
& -\frac{1}{72}(n-1) n(35 n-3) v^{3} \\
& \left.+\frac{3}{2}(n-1) \sqrt{n} v^{2}+\frac{1}{3}(n-1) n v-\sqrt{n}\right) \\
& +u_{1}\left(\frac{1}{2} u_{2}\left((3 n+1) v^{3}+\sqrt{n}(3 n-1) v^{2}-2 \sqrt{n}-6 v\right)\right. \\
& +\frac{1}{96} v\left(\sqrt{n}(n(75 n-38)-5) v^{3}-48(3 n+1) v^{2}\right. \\
& +24(1-5 n) \sqrt{n} v+288)) \\
& +u_{2}\left(3 v-\frac{1}{2}(3 n+1) v^{3}\right) \\
& +\frac{1}{10} v^{3}\left(-5 n\left(n v^{2}-3\right)+v^{2}+5\right) .
\end{aligned}
$$

According to Theorem 1, there exists a 2-dimensional invariant local center manifold $W^{c}(\mathbf{0})$ of $(239), W^{c}(\mathbf{0})=$ $\left\{(\mathbf{u}, v) \in \mathbb{R}^{2} \times \mathbb{R}: v=h(\mathbf{u})\right\}$, satisfying $\mathbf{h}(\mathbf{0})=0, D h(\mathbf{0})$ $=0,|\mathbf{u}|<\delta$ for $\delta$ sufficiently small. The restriction of (239) to the center manifold is $\frac{d \mathbf{u}}{d N}=\mathbf{f}(\mathbf{u}, h(\mathbf{u}))$, where the function $h$ (u) satisfies (A.10):

$D h(\mathbf{u}) \cdot \mathbf{f}(\mathbf{u}, h(\mathbf{u}))+3 h(\mathbf{u})-g(\mathbf{u}, h(\mathbf{u}))=0$.

According to Theorem 3, the system (240) can be solved approximately by expanding $h(\mathbf{u})$ in Taylor series at $\mathbf{u}=\mathbf{0}$. We propose the ansatz

$$
\begin{aligned}
& h\left(u_{1}, u_{2}\right)=a_{1} u_{1}^{2}+a_{10} u_{1} u_{2}^{2}+a_{11} u_{1}^{2} u_{2}^{2}+a_{12} u_{1}^{3} u_{2}^{2}+a_{13} u_{2}^{3} \\
& +a_{14} u_{1} u_{2}^{3}+a_{15} u_{1}^{2} u_{2}^{3}+a_{16} u_{2}^{4}+a_{17} u_{1} u_{2}^{4}+a_{18} u_{2}^{5}+a_{2} u_{1}^{3} \\
& +a_{3} u_{1}^{4}+a_{4} u_{1}^{5}+a_{5} u_{1} u_{2}+a_{6} u_{1}^{2} u_{2}+a_{7} u_{1}^{3} u_{2}+a_{8} u_{1}^{4} u_{2} \\
& +a_{9} u_{2}^{2}+\mathscr{O}\left(|\mathbf{u}|^{6}\right)
\end{aligned}
$$

By comparing the coefficients of the equal powers of $u_{1}, u_{2}$ we find the non-null coefficients

$$
\begin{aligned}
& a_{1}=-\frac{\sqrt{n}}{3}, \quad a_{2}=-\frac{1}{324} \sqrt{n}(n(3 n-7)+108), \\
& a_{3}=-\frac{1}{108} \sqrt{n}(n(3 n-7)+36), \\
& a_{4}=-\frac{\sqrt{n}\left(n\left(n\left(65 n^{2}-570 n+7561\right)-15120\right)+38880\right)}{116640}, \\
& a_{5}=-\frac{\sqrt{n}}{3}, \quad a_{6}=-\frac{\sqrt{n}}{3}, \quad a_{7}=\frac{1}{108} \sqrt{n}(n(5 n+7)-36), \\
& a_{8}=\frac{1}{36}(n-1) \sqrt{n}(5 n+12), \quad a_{10}=-\frac{\sqrt{n}}{3}, \quad a_{11}=-\frac{\sqrt{n}}{3},
\end{aligned}
$$


$a_{12}=\frac{1}{54} \sqrt{n}(n(15 n+7)-18), \quad a_{14}=-\frac{\sqrt{n}}{3}, a_{15}=-\frac{\sqrt{n}}{3}$,

$a_{17}=-\frac{\sqrt{n}}{3}$.

Therefore, $-\frac{\sqrt{n}\left(n\left(n\left(65 n^{2}-570 n+7561\right)-15120\right)+38880\right) u_{1}^{5}}{116640}+\frac{1}{36}(n-1) \sqrt{n}(5 n$ $+12) u_{1}^{4} u_{2}-\frac{1}{108} \sqrt{n}(n(3 n-7)+36) u_{1}^{4}+\frac{1}{54} \sqrt{n}(n(15 n+7)-$ 18) $u_{1}^{3} u_{2}^{2}+\frac{1}{108} \sqrt{n}(n(5 n+7)-36) u_{1}^{3} u_{2}-\frac{1}{324} \sqrt{n}(n(3 n-$ 7) + 108) $u_{1}^{3}-\frac{1}{3} \sqrt{n} u_{1}^{2} u_{2}^{3}-\frac{1}{3} \sqrt{n} u_{1}^{2} u_{2}^{2}-\frac{1}{3} \sqrt{n} u_{1}^{2} u_{2}-\frac{\sqrt{n} u_{1}^{2}}{3}-$ $\frac{1}{3} \sqrt{n} u_{1} u_{2}^{4}-\frac{1}{3} \sqrt{n} u_{1} u_{2}^{3}-\frac{1}{3} \sqrt{n} u_{1} u_{2}^{2}-\frac{1}{3} \sqrt{n} u_{1} u_{2}$, and the center manifold can be expressed as $\theta=\frac{5}{18} n^{5 / 2} Q^{2} T^{3}+$ $\frac{7}{54} n^{3 / 2} Q^{2} T^{3}-\frac{5}{36} n^{5 / 2} Q T^{4}-\frac{7}{36} n^{3 / 2} Q T^{4}-\frac{65}{108} n^{5 / 2} Q T^{3}-$ $\frac{35}{108} n^{3 / 2} Q T^{3}-\frac{13 n^{9 / 2} T^{5}}{23328}+\frac{19 n^{7 / 2} T^{5}}{3888}-\frac{7561 n^{5 / 2} T^{5}}{116640}+\frac{7}{54} n^{3 / 2} T^{5}+$ $\frac{1}{9} n^{5 / 2} T^{4}+\frac{7}{27} n^{3 / 2} T^{4}+\frac{17}{54} n^{5 / 2} T^{3}+\frac{35}{162} n^{3 / 2} T^{3}-\frac{1}{3} \sqrt{n} Q^{4} T+$ $\frac{1}{3} \sqrt{n} Q^{3} T^{2}+\frac{5}{3} \sqrt{n} Q^{3} T-\frac{1}{3} \sqrt{n} Q^{2} T^{3}-\frac{4}{3} \sqrt{n} Q^{2} T^{2}-$ $\frac{10}{3} \sqrt{n} Q^{2} T+\frac{1}{3} \sqrt{n} Q T^{4}+\sqrt{n} Q T^{3}+2 \sqrt{n} Q T^{2}+\frac{10}{3} \sqrt{n} Q T-$ $\frac{\sqrt{n} T^{5}}{3}-\frac{2 \sqrt{n} T^{4}}{3}-\sqrt{n} T^{3}-\frac{4 \sqrt{n} T^{2}}{3}-\frac{5 \sqrt{n} T}{3}$. The dynamics on the center manifold is given by

$$
\begin{aligned}
\frac{d u_{1}}{d N}= & \frac{1}{27} n u_{1}^{3}\left(9\left(u_{2}^{2}+u_{2}+1\right)-n u_{1}^{2}\right), \\
\frac{d u_{2}}{d N}= & \frac{4}{3} n^{2} u_{1}^{2} u_{2}^{3}+n^{2} u_{1}^{2}\left(u_{1}+1\right) u_{2}^{2} \\
& +\frac{2}{27} n^{2} u_{1}^{2} u_{2}\left(u_{1}\left(9-(n-9) u_{1}\right)+9\right) .
\end{aligned}
$$

For which the origin is unstable (see Fig. 9). Using the Theorem 2, we conclude that the center manifold of origin for the system (242), (243), and the origin are unstable (saddle).
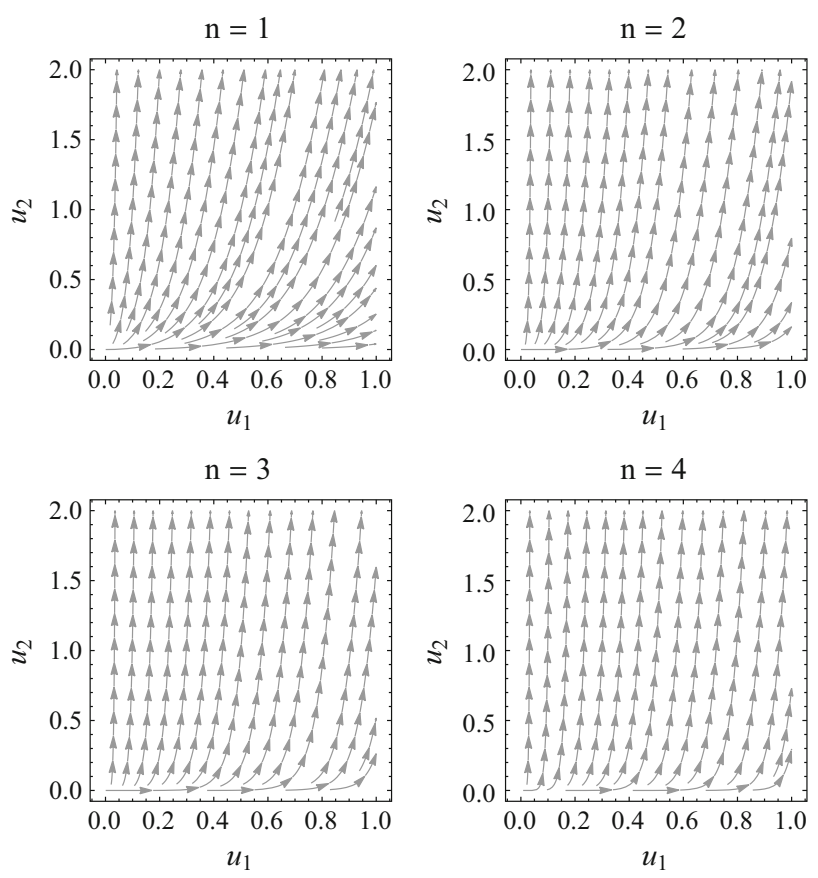

Fig. 9 Numerical solutions of the system (242), (243)

and taking Taylor series near $\left(u_{1}, u_{2}, v\right)=(0,0,0)$ up to fifth order we obtain the system (233), (234), (235) can be written into its Jordan canonical form:

$$
\left(\begin{array}{l}
\frac{d u_{1}}{d N} \\
\frac{d u_{2}}{d N} \\
\frac{d v}{d N}
\end{array}\right)=\left(\begin{array}{ccc}
0 & 0 & 0 \\
0 & 0 & 0 \\
0 & 0 & -3 Q^{*}
\end{array}\right)\left(\begin{array}{c}
u_{1} \\
u_{2} \\
v
\end{array}\right)+\left(\begin{array}{l}
f_{1}^{*}(\mathbf{u}, v) \\
f_{2}^{*}(\mathbf{u}, v) \\
g^{*}(\mathbf{u}, v)
\end{array}\right),
$$

where

$$
\begin{aligned}
& f_{1}^{*}(\mathbf{u}, v)=\frac{u_{1}\left(\sqrt{n} u_{1}-3 v\right)^{2}\left(-(3 n-1)\left(\sqrt{n} u_{1}-3 v\right)^{2}+54 Q^{* 2}\left(u_{1}-1\right)^{2}+54 Q^{*}\left(2 u_{1}-1\right) u_{2}\right)}{162 Q^{* 3}}, \\
& f_{2}^{*}(\mathbf{u}, v)=-\frac{n\left(\sqrt{n} u_{1}-3 v\right)^{2}\left(Q^{* 2}\left(u_{1}-1\right)\left((3 n-1)\left(\sqrt{n} u_{1}-3 v\right)^{2}-54\left(u_{2}^{2}-1\right)\right)+2(3 n-1) Q^{*} u_{2}\left(\sqrt{n} u_{1}-3 v\right)^{2}-(3 n-1)\left(u_{1}-1\right)\left(\sqrt{n} u_{1}-3 v\right)^{2}-54 Q^{* 4}\left(u_{1}-1\right)+108 Q^{* 3}\left(u_{1}-1\right) u_{2}\right)}{162 Q^{* 4}}, \\
& g^{*}(\mathbf{u}, v)=\frac{\left(1-5 n^{2}\right) v^{5}}{10 Q^{* 3}}+\frac{v\left(u_{1}\left(n^{2}((38-15 n) n-7) u_{1}^{3}-108 n Q^{* 2} u_{1}\left(n\left(3 u_{1}-2\right)+u_{1}\left(4 u_{1}-7\right)+2\right)+1944 Q^{* 4}\right)+108 Q^{*} u_{2}\left(n u_{1}^{2}\left(3 n\left(u_{1}-1\right)-7 u_{1}+3\right)-18 Q^{* 2}\left(u_{1}-1\right)\right)\right)}{648 Q^{* 3}} \\
& +\frac{\sqrt{n} u_{1}\left(u_{1}\left(n^{2}(5 n(11 n-38)+39) u_{1}^{3}+360 n Q^{* 2} u_{1}\left(n\left(6 u_{1}-3\right)+2 u_{1}\left(6 u_{1}-11\right)+7\right)-38880 Q^{* 4}\right)+720 Q^{*} u_{2}\left(n u_{1}^{2}\left(-3 n\left(u_{1}-1\right)+11 u_{1}-5\right)+54 Q^{* 2}\left(u_{1}-1\right)\right)\right)}{38880 Q^{* 3}} \\
& +\frac{\sqrt{n}(n(75 n-38)-5) u_{1} v^{4}}{96 Q^{* 3}}-\frac{v^{3}\left(36(3 n+1) Q^{* 2}\left(u_{1}-1\right)-36(3 n+1) Q^{*}\left(u_{1}-1\right) u_{2}+(n-1) n(35 n-3) u_{1}^{2}\right)}{72 Q^{* 3}} \\
& +\frac{\sqrt{n} u_{1} v^{2}\left(108 Q^{* 2}\left(6(n-1) u_{1}-5 n+4 u_{1}^{2}+1\right)-216 Q^{*} u_{2}\left(3 n\left(u_{1}-1\right)-3 u_{1}+1\right)+n(n(65 n-114)+17) u_{1}^{2}\right)}{432 Q^{* 3}}
\end{aligned}
$$

Proof Part (ii) More general, by introducing the linear transformation

$$
\begin{aligned}
& \left(u_{1}, u_{2}, v\right)=\left(T, Q^{*}-Q, \frac{1}{3}\left(3 Q^{*} \theta+\sqrt{n} T\right)\right), \\
& \times u_{1} \in[0,1], u_{2} \in\left[1-\left|Q^{*}\right|, 1+\left|Q^{*}\right|\right] \subset[0,2],
\end{aligned}
$$

By Theorem 1, exists a 2-dimensional local center manifold of (239), $W^{c}(\mathbf{0})=\left\{(\mathbf{u}, v) \in \mathbb{R}^{2} \times \mathbb{R}: v=h(\mathbf{u})\right\}$, satisfying $\mathbf{h}(\mathbf{0})=0, \quad D h(\mathbf{0})=0, \quad|\mathbf{u}|<\delta$ for $\delta$ sufficiently small. The restriction of (245) to the center manifold is $\frac{d \mathbf{u}}{d N}=\mathbf{f}(\mathbf{u}, h(\mathbf{u}))$, where the function $h(\mathbf{u})$ that satisfies (A.10): 
$D h(\mathbf{u}) \cdot \mathbf{f}(\mathbf{u}, h(\mathbf{u}))+3 Q^{*} h(\mathbf{u})-g(\mathbf{u}, h(\mathbf{u}))=0 . \quad(246)$

Replacing (241) in (246) we find the non-null coefficients

$$
\begin{aligned}
& a_{1}=-\frac{\sqrt{n}}{3}, a_{2}=\frac{\sqrt{n}\left(12 n^{2}+(7-15 n) n Q^{* 2}-108 Q^{* 4}\right)}{324 Q^{* 4}}, a_{3}=\frac{\sqrt{n}\left(12 n^{2}+(7-15 n) n Q^{* 2}-36 Q^{* 4}\right)}{108 Q^{* 4}}, \\
& a_{4}=\frac{\sqrt{n}\left(-3360 n^{4}+120 n^{3}(55 n-27) Q^{* 2}+n^{2}(5(762-661 n) n+24839) Q^{* 4}-2160 n(15 n-7) Q^{* 6}-38880 Q^{* 8}\right)}{116640 Q^{* 8}}, \\
& a_{5}=-\frac{\sqrt{n}}{3 Q^{*}}, a_{6}=-\frac{\sqrt{n}}{3 Q^{*}}, a_{7}=\frac{\sqrt{n}\left(20 n^{2}+(7-15 n) n Q^{* 2}-36 Q^{* 4}\right)}{108 Q^{* 5}}, a_{8}=\frac{\sqrt{n}\left(20 n^{2}+(7-15 n) n Q^{* 2}-12 Q^{* 4}\right)}{36 Q^{* 5}}, \\
& a_{10}=-\frac{\sqrt{n}}{3 Q^{* 2}}, a_{11}=-\frac{\sqrt{n}}{3 Q^{* 2}}, a_{12}=\frac{\sqrt{n}\left(30 n^{2}+(7-15 n) n Q^{* 2}-18 Q^{* 4}\right)}{54 Q^{* 6}}, \quad a_{14}=-\frac{\sqrt{n}}{3 Q^{* 3}}, a_{15}=-\frac{\sqrt{n}}{3 Q^{* 3}}, a_{17}=-\frac{\sqrt{n}}{3 Q^{* 4}} .
\end{aligned}
$$

Therefore,

$$
\begin{aligned}
& h\left(u_{1}, u_{2}\right)=\frac{\sqrt{n} u_{1}^{4}\left(12 n^{2}+(7-15 n) n Q^{* 2}-36 Q^{* 4}\right)}{108 Q^{* 4}}+\frac{\sqrt{n} u_{1}^{3}\left(12 n^{2}+(7-15 n) n Q^{* 2}-108 Q^{* 4}\right)}{324 Q^{* 4}} \\
& +\frac{\sqrt{n} u_{1}^{3} u_{2}^{2}\left(30 n^{2}+(7-15 n) n Q^{* 2}-18 Q^{* 4}\right)}{54 Q^{* 6}}+\frac{\sqrt{n} u_{1}^{4} u_{2}\left(20 n^{2}+(7-15 n) n Q^{* 2}-12 Q^{* 4}\right)}{36 Q^{* 5}} \\
& +\frac{\sqrt{n} u_{1}^{3} u_{2}\left(20 n^{2}+(7-15 n) n Q^{* 2}-36 Q^{* 4}\right)}{108 Q^{* 5}} \\
& +\frac{\sqrt{n} u_{1}^{5}\left(-3360 n^{4}+120 n^{3}(55 n-27) Q^{* 2}+n^{2}(5(762-661 n) n+24839) Q^{* 4}-2160 n(15 n-7) Q^{* 6}-38880 Q^{* 8}\right)}{116640 Q^{* 8}} \\
& -\frac{\sqrt{n} u_{1} u_{2}^{4}}{3 Q^{* 4}}-\frac{\sqrt{n} u_{1}^{2} u_{2}^{3}}{3 Q^{* 3}}-\frac{\sqrt{n} u_{1} u_{2}^{3}}{3 Q^{* 3}}-\frac{\sqrt{n} u_{1}^{2} u_{2}^{2}}{3 Q^{* 2}}-\frac{\sqrt{n} u_{1} u_{2}^{2}}{3 Q^{* 2}}-\frac{\sqrt{n} u_{1}^{2} u_{2}}{3 Q^{*}}-\frac{\sqrt{n} u_{1} u_{2}}{3 Q^{*}}-\frac{\sqrt{n} u_{1}^{2}}{3} .
\end{aligned}
$$

Finally, the center manifold can be expressed as

$$
\begin{aligned}
\theta= & \frac{5 n^{5 / 2} Q^{2} T^{3}}{9 Q^{* 7}}-\frac{5 n^{5 / 2} Q^{2} T^{3}}{18 Q^{* 5}}+\frac{7 n^{3 / 2} Q^{2} T^{3}}{54 Q^{* 5}} \\
& -\frac{5 n^{5 / 2} Q T^{4}}{9 Q^{* 6}}-\frac{35 n^{5 / 2} Q T^{3}}{27 Q^{* 6}}+\frac{5 n^{5 / 2} Q T^{4}}{12 Q^{* 4}} \\
& -\frac{7 n^{3 / 2} Q T^{4}}{36 Q^{* 4}}+\frac{25 n^{5 / 2} Q T^{3}}{36 Q^{* 4}}-\frac{35 n^{3 / 2} Q T^{3}}{108 Q^{* 4}} \\
& -\frac{7 n^{9 / 2} T^{5}}{243 Q^{* 9}}+\frac{55 n^{9 / 2} T^{5}}{972 Q^{* 7}}-\frac{n^{7 / 2} T^{5}}{36 Q^{* 7}} \\
& -\frac{661 n^{9 / 2} T^{5}}{23328 Q^{* 5}}+\frac{127 n^{7 / 2} T^{5}}{3888 Q^{* 5}}+\frac{24839 n^{5 / 2} T^{5}}{116640 Q^{* 5}} \\
& +\frac{2 n^{5 / 2} T^{4}}{3 Q^{* 5}}+\frac{7 n^{5 / 2} T^{3}}{9 Q^{* 5}}-\frac{5 n^{5 / 2} T^{5}}{18 Q^{* 3}} \\
& +\frac{7 n^{3 / 2} T^{5}}{54 Q^{* 3}}-\frac{5 n^{5 / 2} T^{4}}{9 Q^{* 3}}+\frac{7 n^{3 / 2} T^{4}}{27 Q^{* 3}}
\end{aligned}
$$

$$
\begin{aligned}
& -\frac{25 n^{5 / 2} T^{3}}{54 Q^{* 3}}+\frac{35 n^{3 / 2} T^{3}}{162 Q^{* 3}}-\frac{\sqrt{n} Q^{4} T}{3 Q^{* 5}} \\
& +\frac{\sqrt{n} Q^{3} T^{2}}{3 Q^{* 4}}+\frac{5 \sqrt{n} Q^{3} T}{3 Q^{* 4}}-\frac{\sqrt{n} Q^{2} T^{3}}{3 Q^{* 3}} \\
& -\frac{4 \sqrt{n} Q^{2} T^{2}}{3 Q^{* 3}}-\frac{10 \sqrt{n} Q^{2} T}{3 Q^{* 3}}+\frac{\sqrt{n} Q T^{4}}{3 Q^{* 2}} \\
& +\frac{\sqrt{n} Q T^{3}}{Q^{* 2}}+\frac{2 \sqrt{n} Q T^{2}}{Q^{* 2}}+\frac{10 \sqrt{n} Q T}{3 Q^{* 2}} \\
& -\frac{\sqrt{n} T^{5}}{3 Q^{*}}-\frac{2 \sqrt{n} T^{4}}{3 Q^{*}}-\frac{\sqrt{n} T^{3}}{Q^{*}} \\
& -\frac{4 \sqrt{n} T^{2}}{3 Q^{*}}-\frac{5 \sqrt{n} T}{3 Q^{*}} .
\end{aligned}
$$

The dynamics on the center manifold is given by 


$$
\begin{aligned}
\frac{d u_{1}}{d N}= & \frac{n u_{1}^{3}\left(Q^{* 2}\left(n(2 n-1) u_{1}^{2}+9\left(Q^{* 2}+Q^{*} u_{2}+u_{2}^{2}\right)\right)-2 n^{2} u_{1}^{2}\right)}{27 Q^{* 5}}, \\
\frac{d u_{2}}{d N}= & \frac{4 n^{2} u_{1}^{2} u_{2}^{3}}{3 Q^{* 5}}+\frac{n^{2} u_{1}^{2}\left(u_{1}+1\right) u_{2}^{2}}{Q^{* 4}}+\frac{2 n^{2} u_{1}^{2} u_{2}\left(Q^{* 4}\left(u_{1}\left(\left(-2 n^{2}+n+9\right) u_{1}+9\right)+9\right)-6 n^{2} u_{1}^{2}+2 n(4 n-1) Q^{* 2} u_{1}^{2}\right)}{27 Q^{* 7}} \\
& -\frac{n^{2}\left(Q^{* 2}-1\right) u_{1}^{2}\left(-2 n^{2} u_{1}^{2}\left(3 u_{1}+1\right)+n(2 n-1) Q^{* 2} u_{1}^{2}\left(3 u_{1}+1\right)+9 Q^{* 4}\left(u_{1}+1\right)\left(u_{1}^{2}+1\right)\right)}{27 Q^{* 6}} .
\end{aligned}
$$

In the Fig. 10 are presented some numerical solutions of the system (247), (248) for $n=1,4$ and $Q^{*}= \pm 1$. The plot illustrates the generic feature that for $Q^{*}>0$ (respectively, $Q^{*}<0$ ), the center manifold is unstable (respectively, stable), but in this case the third eigenvalue is $-3 Q^{*}<0$ (respectively, $-3 Q^{*}>0$ ). That is, the origin is a saddle.

\subsection{E-models}

In this example the dynamical system (209), (210), (211) is reduced to

$$
\begin{aligned}
& \frac{d X}{d \tau}=\left(X^{2}-1\right)\left(3 \sqrt{1-U^{2}} X-\sqrt{6} \tan \left(\frac{\pi S}{2}\right)\right), \\
& \frac{d U}{d \tau}=3 U \sqrt{1-U^{2}} X^{2},
\end{aligned}
$$
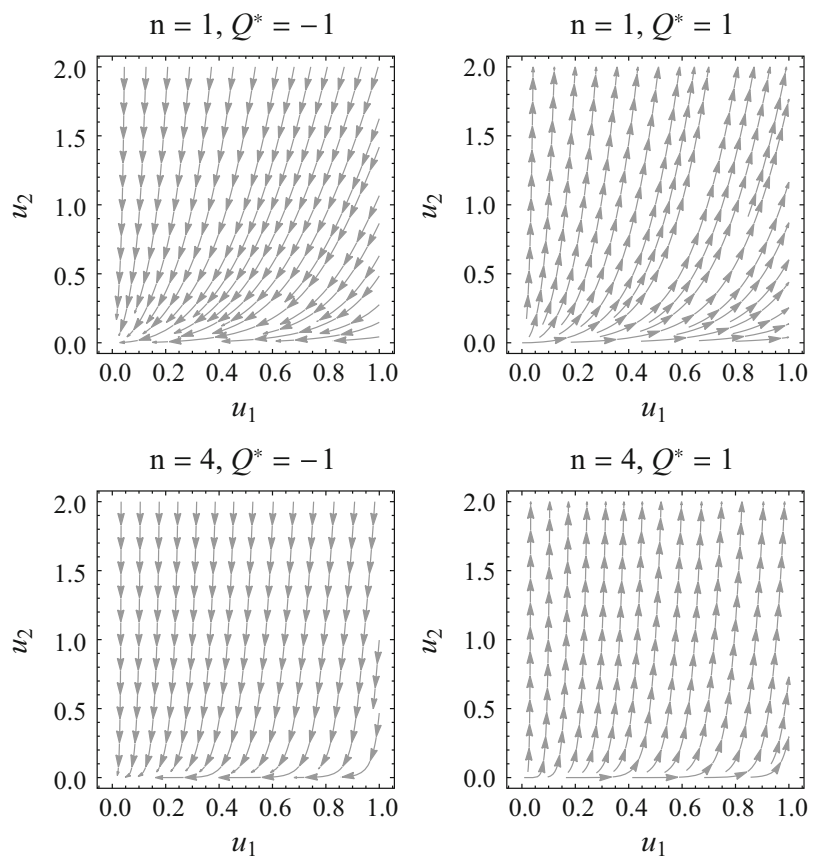

Fig. 10 Numerical solutions of the system (247), (248)

$$
\frac{d S}{d \tau}=\frac{X(-6 \mu \sin (\pi S)-\sqrt{6} \cos (\pi S)+\sqrt{6})}{\pi n},
$$

defined on the compact phase space

$$
\left\{(X, U, S) \in \mathbb{R}^{3}:-1 \leq X \leq 1,-1 \leq U \leq 1,-1 \leq S \leq 1\right\} .
$$

The coordinates equilibrium points of the system (249), (250), (251) and their stability conditions are summarized as follows:

$-(x, U, S)=\left(0, U_{c}, 0\right)$ with eigenvalues $\{0,0$, $\left.-3 \sqrt{1-U_{c}^{2}}\right\}$. Non-hyperbolic with a 1 dimensional stable manifold.

$-(x, U, S) \quad=\quad(-1,0,0) \quad$ with eigenvalues $\left\{3,6, \frac{6 \mu}{n}\right\}$. It is a source.

$-(x, U, S)=\left(-1,0, \frac{2 \arctan (\sqrt{6} \mu)}{\pi}\right)$ with eigenvalues $\left\{3,12 \mu+6,-\frac{6 \mu}{n}\right\}$. It is a saddle.

$-(x, U, S) \quad=\quad(1,0,0) \quad$ with eigenvalues $\left\{3,6,-\frac{6 \mu}{n}\right\}$. It is a saddle.

$-(x, U, S)=\left(1,0, \frac{2 \arctan (\sqrt{6} \mu)}{\pi}\right)$ with eigenvalues $\left\{3,6-12 \mu, \frac{6 \mu}{n}\right\}$. It is a source for $\mu<\frac{1}{2}$.

- $(x, U, S)=(-1,-1,0)$ with eigenvalues $\left\{0,-\infty, \frac{6 \mu}{n}\right\}$. Non-hyperbolic with a 1 dimensional stable manifold.

$-(x, U, S)=\left(-1,-1, \frac{2 \arctan (\sqrt{6} \mu)}{\pi}\right)$ with eigenvalues $\left\{-\infty, 12 \mu,-\frac{6 \mu}{n}\right\}$. It is a saddle.

$-(x, U, S)=(-1,1,0)$ with eigenvalues $\left\{0,-\infty, \frac{6 \mu}{n}\right\}$. Non-hyperbolic. Behaves as saddle.

$-(x, U, S)=\left(-1,1, \frac{2 \arctan (\sqrt{6} \mu)}{\pi}\right)$ with eigenvalues $\left\{-\infty, 12 \mu,-\frac{6 \mu}{n}\right\}$. It is a saddle. 
$-(x, U, S)=(1,-1,0)$ with eigenvalues $\left\{0,-\infty,-\frac{6 \mu}{n}\right\}$. Non-hyperbolic with a 2 dimensional stable manifold.

$-(x, U, S)=\left(1,-1, \frac{2 \arctan (\sqrt{6} \mu)}{\pi}\right)$ with eigenvalues $\left\{-\infty,-12 \mu, \frac{6 \mu}{n}\right\}$. It is a saddle.

$-(X, Z, S)=(1,1,0)$. The eigenvalues are $\left\{0,-\infty,-\frac{6 \mu}{n}\right\}$. Nonhyperbolic with a 2 dimensional stable manifold.

$-(x, U, S)=\left(1,1, \frac{2 \arctan (\sqrt{6} \mu)}{\pi}\right)$ with eigenvalues $\left\{-\infty,-12 \mu, \frac{6 \mu}{n}\right\}$. It is a saddle.

\subsubsection{Alternative compactification}

Introducing the new variables

$$
\begin{aligned}
\hat{H} & =\sqrt{H^{2}+\frac{\mu^{2} \Lambda^{2}}{16(3 \lambda-1)^{2}}}, \\
Q & =\frac{H}{\bar{H}}, \\
\Sigma & =\frac{\dot{\phi}}{2 \sqrt{6} \bar{H}}, \\
Y & =\left(\frac{V(\phi)}{6(3 \lambda-1) \bar{H}^{2}}\right)^{\frac{1}{2 n}}=\hat{T}\left(1-e^{-\sqrt{\frac{2}{3 \alpha}} \phi}\right), \\
\hat{T} & =\left[\frac{V_{0}}{6(3 \lambda-1) \hat{H}^{2}}\right]^{\frac{1}{2 n}},
\end{aligned}
$$

such that

$$
\begin{aligned}
& \dot{\phi}=\frac{2 \Sigma \sqrt{V_{0}} \hat{T}^{-n}}{\sqrt{3 \lambda-1}}, \\
& \phi=-\frac{\sqrt{\frac{2}{3}} n \ln \left(1-\frac{Y}{\hat{T}}\right)}{\mu}, \\
& \Lambda=\frac{2 \sqrt{\frac{2}{3}} \sqrt{3 \lambda-1} \sqrt{1-Q^{2}} \sqrt{V_{0}} \hat{T}^{-n}}{\mu}, \\
& H=\frac{Q \sqrt{V_{0}} \hat{T}^{-n}}{\sqrt{18 \lambda-6}}, \\
& \Sigma+Y^{2 n}=1
\end{aligned}
$$

and the new time variable $\frac{d M}{d t}=H / Q$, we obtain the dynamical system becomes

$$
\begin{aligned}
& \frac{d \Sigma}{d M}=6 \mu Y^{2 n-1}(Y-\hat{T})+Q \Sigma\left((q+1) Q^{2}-3\right), \\
& \frac{d Y}{d M}=\frac{(q+1) Q^{3} Y+6 \mu \Sigma(\hat{T}-Y)}{n}
\end{aligned}
$$

$$
\begin{aligned}
& \frac{d \hat{T}}{d M}=\frac{(q+1) Q^{3} \hat{T}}{n} \\
& \frac{d Q}{d M}=(q+1) Q^{2}\left(Q^{2}-1\right)
\end{aligned}
$$

where

$Q^{2} q=\frac{1}{2}\left(-3 Y^{2 n}-2 Q^{2}+3 \Sigma^{2}+3\right)=3 \Sigma^{2}-Q^{2}$.

Introducing the complementary global transformation

$\Sigma=F(\theta) \sin (\theta), \quad Y=\cos (\theta)$,

$F(\theta)=\sqrt{\frac{1-\cos ^{2 n}(\theta)}{1-\cos ^{2}(\theta)}}$

we obtain the regular unconstrained 3D dynamical system

$\frac{d \theta}{d M}=-\frac{3 Q F(\theta)^{2} \sin (\theta) \cos (\theta)}{n}-\frac{6 \mu F(\theta)(\hat{T}-\cos (\theta))}{n}$,

$\frac{d \hat{T}}{d M}=\frac{3 Q \hat{T}\left(1-\cos ^{2 n}(\theta)\right)}{n}$,

$\frac{d Q}{d M}=3\left(Q^{2}-1\right)\left(1-\cos ^{2 n}(\theta)\right)$.

where the deceleration parameter is expressed as

$Q^{2} q=-3 \cos ^{2 n}(\theta)-Q^{2}+3$.

Introducing the new compact variable $T=\frac{\hat{T}}{1+\hat{T}}$, and the new time derivative $\frac{d \bar{\tau}}{d M}=1+\hat{T}=(1-T)^{-1}$, we obtain:

$$
\begin{aligned}
\frac{d \theta}{d \bar{\tau}} & =-\frac{6 \mu F(\theta)((T-1) \cos (\theta)+T)}{n} \\
& +\frac{3 Q(T-1) F(\theta)^{2} \sin (2 \theta)}{2 n}, \\
\frac{d T}{d \bar{\tau}} & =\frac{3 Q(T-1)^{2} T\left(1-\cos ^{2 n}(\theta)\right)}{n}, \\
\frac{d Q}{d \bar{\tau}} & =-3\left(Q^{2}-1\right)(T-1)\left(1-\cos ^{2 n}(\theta)\right) .
\end{aligned}
$$

The coordinates equilibrium points of the system (272), (273), (274) and their stability conditions are summarized as follows:

${ }{ }_{ \pm} M_{ \pm}: \hat{T}=T=0 ; \Sigma= \pm 1, Q= \pm 1, Y=$ $0 ; \theta= \pm \frac{\pi}{2}+2 k \pi, k \in \mathbb{Z}$, were we have used the Kernel: $\operatorname{sign}(Q) M_{\operatorname{sign}(\Sigma)}$. The eigenvalues of $-M_{+}$are $\left\{-\frac{3}{n},-6,-\frac{6 \mu+3}{n}\right\}$. It is a sink. The eigenvalues of $M_{-}$ are $\left\{-\frac{3}{n},-6, \frac{6 \mu-3}{n}\right\}$. It is a sink for $\mu<\frac{1}{2}$ or a saddle otherwise. The eigenvalues of $+M_{+}$are $\left\{\frac{3}{n}, 6, \frac{3-6 \mu}{n}\right\}$. It 


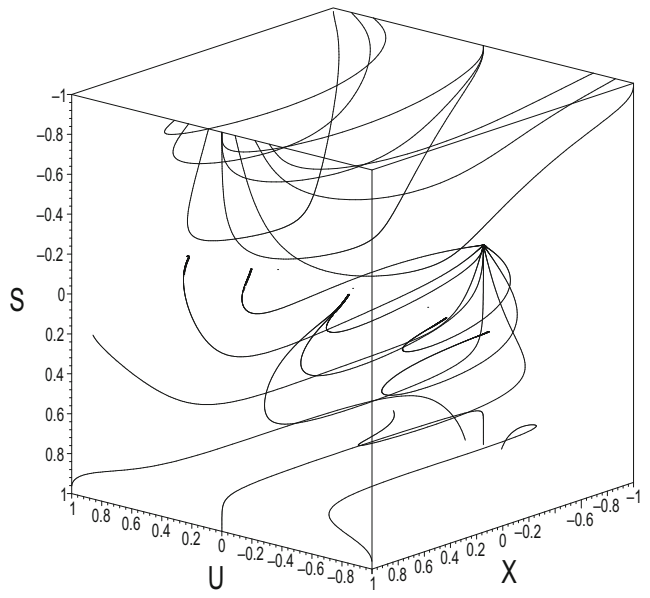

(a)

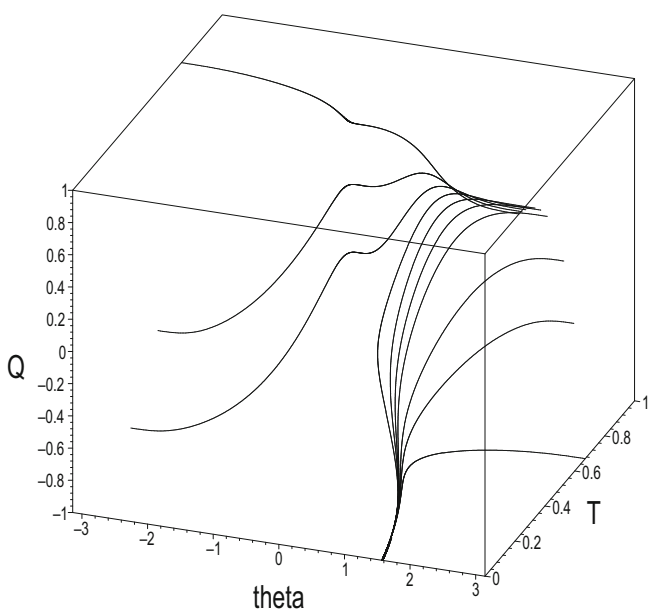

(c)

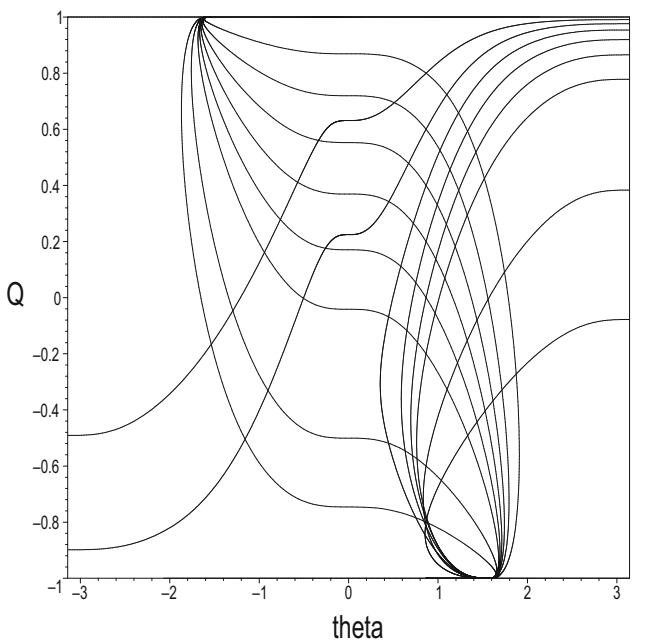

(e)

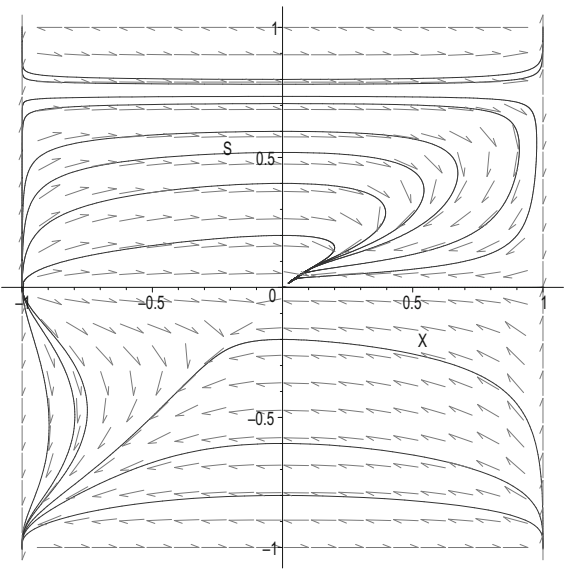

(b)

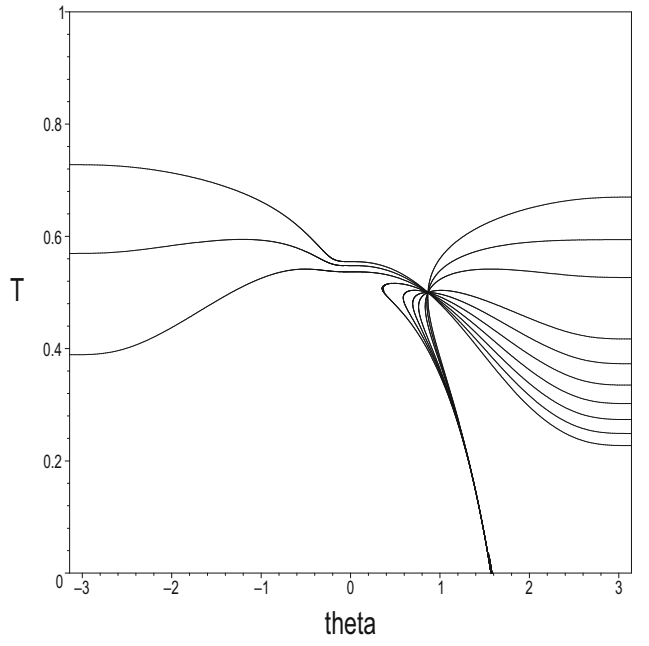

(d)

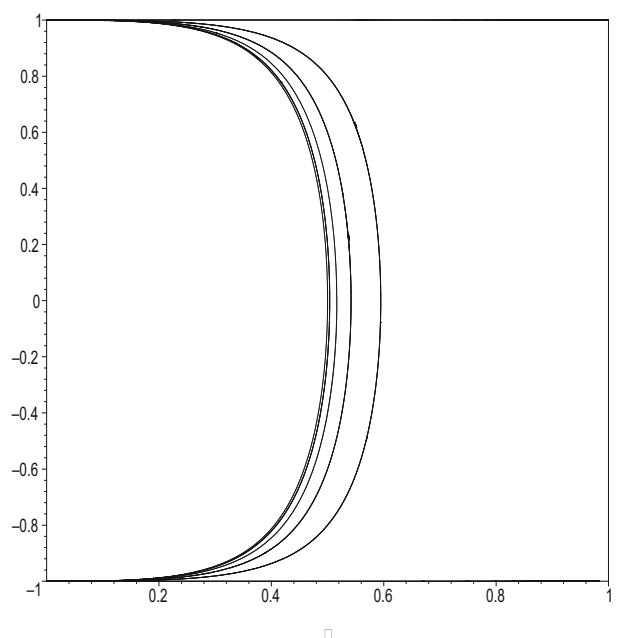

(f)

Fig. 11 a Compact phase portrait of (249), (250), (251) for the choice $n=2, \mu=1$. b Dynamics in the invariant set $Z=0$. c-f Dynamics of (272), (273), (274) and some 2D projections of the solutions for $n=4, \mu=1$ 
is a source for $\mu<\frac{1}{2}$ or a saddle otherwise. The eigenvalues of $+M_{-}$are $\left\{\frac{3}{n}, 6, \frac{6 \mu+3}{n}\right\}$. It is a source.

$-d S^{ \pm}: \hat{T}=1, T=\frac{1}{2} ; \Sigma=0, Q= \pm 1, Y=1 ; \theta=$ $2 k \pi, k \in \mathbb{Z}$. The eigenvalues of $d S^{+}$are $\left\{0,0,-\frac{3}{2}\right\}$. Nonhyperbolic with 1D stable manifold. The eigenvalues of $d S^{-}$are $\left\{0,0, \frac{3}{2}\right\}$. Nonhyperbolic with 1D unstable manifold. More generally, we have $d S\left(Q^{*}\right): \hat{T}=1, T=$ $\frac{1}{2} ; \Sigma=0, Q=Q^{*}, Y=1 ; \theta=2 k \pi, k \in \mathbb{Z}$, with eigenvalues $\left\{0,0,-\frac{3}{2} Q^{*}\right\}$.

- $P L^{ \pm}: \hat{T}=T=0 ; \Sigma= \pm 2 \mu, Q= \pm 1 ; Y=$ $-\left(1-4 \mu^{2}\right)^{\frac{1}{2 n}} ; \theta= \pm \arccos Y$. Exists for $\mu<1 / 2$. The eigenvalues are $\left\{\frac{12 \mu^{2}}{n}, 24 \mu^{2}, 12 \mu^{2}-3\right\}$. It is a saddle.

In the Fig. 11 it is shown (a) a compact phase portrait of the system (249), (250), (251) for the choice $n=2, \mu=1$. (b) Dynamics in the invariant set $Z=0$. In Fig. $11 \mathrm{c}-\mathrm{f}$ it is presented the dynamics of the system (272), (273), (274) and some $2 \mathrm{D}$ projections of the solutions for $n=4, \mu=1$.
5.4.2 Center manifold of the de Sitter solution: for (i) $d S^{+}:(\theta, T, Q)=\left(0, \frac{1}{2}, 1\right)$, (ii) For $d S^{+}\left(Q^{*}\right):(\theta, T, Q)=\left(0, \frac{1}{2}, Q^{*}\right), Q^{*} \neq \pm 1,0$, for Hořava-Lifshitz model with flat universe with $\Lambda \neq 0$ and E-model under the detailed-balance

Proposition 10 (i) The point $d S^{+}:(\theta, T, Q)=\left(0, \frac{1}{2}, 1\right)$ of the system (272), (273), (274) is unstable (saddle). (ii) The line of fixed points $d S_{+}\left(Q^{*}\right):(\theta, T, Q)=\left(0, \frac{1}{2}, Q^{*}\right), Q^{*} \neq$ $\pm 1,0$ of the system (272), (273), (274) is unstable (saddle).

Proof Part (i) Taking the linear transformation

$$
\begin{aligned}
\left(u_{1}, u_{2}, v\right)= & \left(\frac{\mu(4-8 T)}{\sqrt{n}}, \frac{\mu(4-8 T)}{\sqrt{n}}\right. \\
& \left.+Q-1, \theta+\frac{4 \mu(2 T-1)}{\sqrt{n}}\right),
\end{aligned}
$$

and taking Taylor series near $\left(u_{1}, u_{2}, v\right)=(0,0,0)$ up to fifth order we obtain the system (272), (273), (274) can be written into its Jordan canonical form:

$$
\left(\begin{array}{c}
\frac{d u_{1}}{d N} \\
\frac{d u_{2}}{d N} \\
\frac{d v}{d N}
\end{array}\right)=\left(\begin{array}{ccc}
0 & 0 & 0 \\
0 & 0 & 0 \\
0 & 0 & -\frac{3}{2}
\end{array}\right)\left(\begin{array}{c}
u_{1} \\
u_{2} \\
v
\end{array}\right)+\left(\begin{array}{c}
f_{1}(\mathbf{u}, v) \\
f_{2}(\mathbf{u}, v) \\
g(\mathbf{u}, v)
\end{array}\right),
$$

where

$$
\begin{aligned}
f_{1}(\mathbf{u}, v)= & \frac{u_{1}^{5} v\left(-15 n^{3 / 2}-32 \mu^{3}(15(n-1) n+4)+80 \mu^{2}(1-3 n) \sqrt{n}+20 \mu(1-3 n) n\right)}{160 \mu^{2} \sqrt{n}} \\
& +\frac{u_{1}^{4} v^{2}\left(-3 n^{3 / 2}-32 \mu^{3}(15(n-1) n+4)+48 \mu^{2}(1-3 n) \sqrt{n}+12 \mu(1-3 n) n\right)}{64 \mu^{2} \sqrt{n}} \\
& +\frac{u_{1}^{2}\left(3 n^{3 / 2} u_{1}^{3}-32 \mu^{3}\left((3 n-1) u_{1}^{2}-6\right)\left(u_{1}-u_{2}-1\right)+12 \mu n u_{1}^{2}\left(-u_{1}+u_{2}+1\right)+8 \mu^{2} \sqrt{n} u_{1}\left(u_{1}\left((3 n-1) u_{1}+6\right)-6\left(u_{2}+1\right)\right)\right)}{64 \mu^{2} \sqrt{n}} \\
& +\frac{u_{1} v^{3}\left(-16 \mu^{2}\left(15 n^{2} u_{1}^{2}-3 n\left(5 u_{1}^{2}-3 u_{1}+3 u_{2}+3\right)+4 u_{1}^{2}-3 u_{1}+3 u_{2}+3\right)+3(1-3 n) n u_{1}^{2}-12 \mu \sqrt{n}(3 n-1) u_{1}\left(u_{1}-u_{2}-1\right)\right)}{24 \mu \sqrt{n}} \\
& +\frac{u_{1}^{2} v^{4}\left(-3 n^{2}-16 \mu^{2}(15(n-1) n+4)+4 \mu(1-3 n) \sqrt{n}+n\right)}{32 \mu \sqrt{n}} \\
& +\frac{u_{1}^{4} v\left(16 \mu^{2}(3 n-1)+3 n\right)\left(\sqrt{n}\left(u_{2}+1\right)-4 \mu\right)}{32 \mu^{2} \sqrt{n}}+\frac{3 u_{1}^{3} v^{2}\left(16 \mu^{2}(3 n-1)+n\right)\left(\sqrt{n}\left(u_{2}+1\right)-4 \mu\right)}{64 \mu^{2} \sqrt{n}} \\
& +\frac{1}{8} u_{1}^{3} v\left(\frac{\left(u_{2}+1\right)\left(16 \mu^{2}(3 n-1)+3 n\right)}{\mu \sqrt{n}}+12\right)+\frac{3 u_{1}^{2} v^{2}\left(4 \mu \sqrt{n}+16 \mu^{2}(3 n-1)\left(u_{2}+1\right)+n\left(u_{2}+1\right)\right)}{16 \mu \sqrt{n}} \\
& +u_{1}^{2}\left(\frac{6 \mu v}{\sqrt{n}}-\frac{3}{2}\left(u_{2}+1\right) v\right)+\frac{(3 n-1) u_{1} v^{4}\left(\sqrt{n}\left(u_{2}+1\right)-4 \mu\right)}{8 \sqrt{n}}+\frac{3}{4} u_{1} v^{2}\left(\frac{4 \mu}{\sqrt{n}}-u_{2}-1\right) \\
& -\frac{6 \mu u_{1}\left(u_{2}+1\right) v}{\sqrt{n}}-\frac{\mu(15(n-1) n+4) u_{1} v^{5}}{5 \sqrt{n}}+\frac{\mu(3 n-1)\left(u_{2}+1\right) v^{4}}{2 \sqrt{n}}-\frac{3 \mu\left(u_{2}+1\right) v^{2}}{\sqrt{n}},
\end{aligned}
$$




$$
\begin{aligned}
& f_{2}(\mathbf{u}, v)=\frac{1}{64}\left(8(12 n-1) n+\frac{3 n}{\mu^{2}}+\frac{12(2 n-1) \sqrt{n}}{\mu}-8+\frac{32(1-3 n) \mu}{\sqrt{n}}\right) u_{1}^{5} \\
& +\frac{1}{160} v\left(-80(6 n+1) n-\frac{15 n}{\mu^{2}}+\frac{20(n(12 n-7)+1) \sqrt{n}}{\mu}+80-\frac{32(15(n-1) n+4) \mu}{\sqrt{n}}\right) u_{1}^{5} \\
& +\frac{\left(8(3 n-1)\left(u_{2}+1\right) \mu^{2}+4 \sqrt{n}\left(2 n\left(-3 n u_{2}+u_{2}+3\right)+3\right) \mu-3 n(4 n-1)\left(u_{2}+1\right)\right) u_{1}^{4}}{16 \sqrt{n} \mu} \\
& +\frac{v\left(64(1-3 n) \mu^{3}+16 \sqrt{n}(3 n-1)(4 n+1)\left(u_{2}+1\right) \mu^{2}+4 n\left(2 n\left((2-6 n) u_{2}+3\right)-3\right) \mu+3 n^{3 / 2}\left(u_{2}+1\right)\right) u_{1}^{4}}{32 \sqrt{n} \mu^{2}} \\
& +\frac{v^{2}\left(-32(15(n-1) n+4) \mu^{3}-48 \sqrt{n}\left(6 n^{2}+n-1\right) \mu^{2}+12 n(n(12 n-7)+1) \mu-3 n^{3 / 2}\right) u_{1}^{4}}{64 \sqrt{n} \mu^{2}} \\
& +\frac{3}{8}\left(\frac{u_{2}\left(u_{2}+2\right) n^{3 / 2}}{\mu}-2(4 n+1)\left(u_{2}+1\right)+\frac{8 \mu}{\sqrt{n}}\right) u_{1}^{3} \\
& +\frac{v^{3}\left(-16(15(n-1) n+4) \mu^{2}-12 \sqrt{n}\left(6 n^{2}+n-1\right) \mu+3 n(n(12 n-7)+1)\right) u_{1}^{3}}{24 \sqrt{n} \mu} \\
& +\frac{v\left(16(3 n-1)\left(u_{2}+1\right) \mu^{2}+4 \sqrt{n}\left(2 n\left(3-(3 n-1) u_{2}\left(u_{2}+2\right)\right)+3\right) \mu-3 n(4 n-1)\left(u_{2}+1\right)\right) u_{1}^{3}}{8 \sqrt{n} \mu} \\
& +\frac{3 v^{2}\left(64(1-3 n) \mu^{3}+16 \sqrt{n}(3 n-1)(4 n+1)\left(u_{2}+1\right) \mu^{2}+4 n\left(4(1-3 n) u_{2} n+2 n-1\right) \mu+n^{3 / 2}\left(u_{2}+1\right)\right) u_{1}^{3}}{64 \sqrt{n} \mu^{2}} \\
& +\frac{3}{4} v\left(\frac{u_{2}\left(u_{2}+2\right) n^{3 / 2}}{\mu}-2(4 n+1)\left(u_{2}+1\right)+\frac{8 \mu}{\sqrt{n}}\right) u_{1}^{2}+\left(\frac{3}{2} n u_{2}\left(u_{2}+2\right)-\frac{3\left(u_{2}+1\right) \mu}{\sqrt{n}}\right) u_{1}^{2} \\
& -(3 n-1) v^{3}\left(u_{2} n^{2}-(4 n+1)\left(u_{2}+1\right) \mu \sqrt{n}+4 \mu^{2}\right) u_{1}^{2} \\
& 2 \sqrt{n} \mu \\
& +\frac{v^{4}\left(-16(15(n-1) n+4) \mu^{2}-4 \sqrt{n}\left(6 n^{2}+n-1\right) \mu+n(n(12 n-7)+1)\right) u_{1}^{2}}{32 \sqrt{n} \mu} \\
& +\frac{3 v^{2}\left(16(3 n-1)\left(u_{2}+1\right) \mu^{2}+4 \sqrt{n}\left(-6 u_{2}\left(u_{2}+2\right) n^{2}+2\left(u_{2}+1\right)^{2} n+1\right) \mu-n(4 n-1)\left(u_{2}+1\right)\right) u_{1}^{2}}{15(n-1) n+4) v^{5} \mu u_{1}}
\end{aligned}
$$

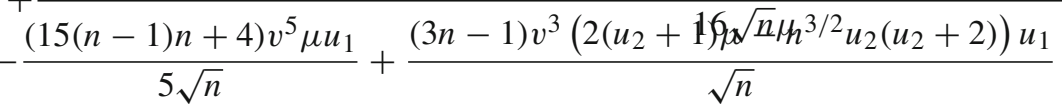

$$
\begin{aligned}
& +\left(3 n u_{2}\left(u_{2}+2\right) v-\frac{6\left(u_{2}+1\right) v \mu}{\sqrt{n}}\right) u_{1}-\frac{(3 n-1) v^{4}\left(u_{2} n^{2}-(4 n+1)\left(u_{2}+1\right) \mu \sqrt{n}+4 \mu^{2}\right) u_{1}}{8 \sqrt{n} \mu} \\
& +\frac{3 v^{2}\left(u_{2}\left(u_{2}+2\right) n^{2}-2(4 n+1)\left(u_{2}+1\right) \mu \sqrt{n}+8 \mu^{2}\right) u_{1}}{8 \sqrt{n} \mu}+\frac{3}{2} n u_{2}\left(u_{2}+2\right) v^{2}-\frac{3\left(u_{2}+1\right) v^{2} \mu}{\sqrt{n}} \\
& -\frac{(3 n-1) v^{4}\left(n^{3 / 2} u_{2}\left(u_{2}+2\right)-2\left(u_{2}+1\right) \mu\right)}{4 \sqrt{n}}, \\
& g(\mathbf{u}, v)=\frac{u_{1}^{4} v\left(-3 n^{3 / 2}+64 \mu^{3}(3 n-1)-2 \mu^{2} \sqrt{n}(n(15 n+28)-13)+6 \mu(1-3 n) n\right)}{32 \mu^{2} \sqrt{n}}
\end{aligned}
$$$$
+\frac{3 u_{1}^{2} v^{2}\left(3 n^{2}\left(u_{2}+1\right)-4 \mu(3 n+2) \sqrt{n}+4 \mu^{2}\left(-3 n\left(4 u_{2}+3\right)+4 u_{2}+2\right)\right)}{16 \mu \sqrt{n}}+\frac{1}{20}\left(1-5 n^{2}\right) v^{5}
$$$$
-\frac{u_{1}^{3} v^{2}\left(3 n^{3 / 2}+36 \mu n^{2}+192 \mu^{3}(1-3 n)+2 \mu^{2} \sqrt{n}(n(65 n+84)-43)\right)}{64 \mu^{2} \sqrt{n}}
$$$$
+\frac{1}{320} u_{1}^{5}\left(-\frac{15 n}{\mu^{2}}+\frac{20 \sqrt{n}(2-3 n)}{\mu}+\frac{160 \mu(3 n-1)}{\sqrt{n}}-5 n(11 n+28)+61\right)
$$$$
+\frac{u_{1}^{4}\left(-4 \mu \sqrt{n}(3 n+4)+2 \mu^{2}\left(-3 n\left(4 u_{2}+3\right)+4 u_{2}+2\right)+n(3 n-2)\left(u_{2}+1\right)\right)}{16 \mu \sqrt{n}}
$$$$
+u_{1}^{3}\left(-\frac{3 \mu}{\sqrt{n}}+\frac{3 \sqrt{n}}{8 \mu}+\frac{3}{8} n\left(2 u_{2}+1\right)+u_{2}+1\right)
$$ 


$$
\begin{aligned}
& +\frac{u_{1}^{3} v\left(-36 \mu \sqrt{n}(n+1)+8 \mu^{2}\left(-3 n\left(4 u_{2}+3\right)+4 u_{2}+2\right)+3 n(3 n-1)\left(u_{2}+1\right)\right)}{16 \mu \sqrt{n}} \\
& +\frac{3}{8} u_{1}^{2}\left(-\frac{\sqrt{n}\left(u_{2}+1\right)}{\mu}+\frac{4\left(\mu+2 \mu u_{2}\right)}{\sqrt{n}}+4\right)+\frac{3}{8} u_{1}^{2} v\left(\frac{\sqrt{n}}{\mu}-\frac{16 \mu}{\sqrt{n}}+6 n u_{2}+4 n+6 u_{2}+6\right) \\
& -\frac{u_{1}^{2} v^{3}\left(32 \mu^{2}(1-3 n)+\mu \sqrt{n}(7 n(5 n+4)-17)+n(3 n+1)\right)}{16 \mu \sqrt{n}} \\
& +v^{4}\left(\frac{\mu\left(3 n\left(4 u_{1}-4 u_{2}-3\right)-4 u_{1}+4 u_{2}+2\right)}{8 \sqrt{n}}-\frac{1}{64}(n(75 n+28)-25) u_{1}\right) \\
& +\frac{u_{1} v^{3}\left(-4 \mu \sqrt{n}(3 n+1)+8 \mu^{2}\left(-3 n\left(4 u_{2}+3\right)+4 u_{2}+2\right)+n(3 n+1)\left(u_{2}+1\right)\right)}{16 \mu \sqrt{n}} \\
& +\frac{3}{8} u_{1} v^{2}\left(-\frac{8 \mu}{\sqrt{n}}+6 n u_{2}+5 n+4 u_{2}+4\right)+\frac{3}{8} u_{1} v\left(-\frac{\sqrt{n}\left(u_{2}+1\right)}{\mu}+\frac{8\left(\mu+2 \mu u_{2}\right)}{\sqrt{n}}+4\right) \\
& +\frac{1}{4}(3 n+1)\left(u_{2}+1\right) v^{3}+\frac{3 \mu\left(2 u_{2}+1\right) v^{2}}{2 \sqrt{n}}-\frac{3 u_{1} u_{2}}{2}-\frac{3 u_{2} v}{2} .
\end{aligned}
$$

According to Theorem 1, there exists a 2-dimensional invariant local center manifold $W^{c}(\mathbf{0})$ of $(276), W^{c}(\mathbf{0})=$ $\left\{(\mathbf{u}, v) \in \mathbb{R}^{2} \times \mathbb{R}: v=h(\mathbf{u})\right\}$, satisfying $\mathbf{h}(\mathbf{0})=0, \quad D h(\mathbf{0})$ $=0,|\mathbf{u}|<\delta$ for $\delta$ sufficiently small. The restriction of (276) to the center manifold is

$\frac{d \mathbf{u}}{d N}=\mathbf{f}(\mathbf{u}, h(\mathbf{u}))$,

where the function $h(\mathbf{u})$ that defines the local center manifold satisfies (A.10):

$D h(\mathbf{u}) \cdot \mathbf{f}(\mathbf{u}, h(\mathbf{u}))+\frac{3}{2} h(\mathbf{u})-g(\mathbf{u}, h(\mathbf{u}))=0$.

$a_{1}=\frac{\mu}{\sqrt{n}}-\frac{\sqrt{n}}{4 \mu}+1, \quad a_{2}=\frac{6 \mu^{2}}{n}+\frac{1}{16}\left(\frac{1}{\mu^{2}}+4\right) n+\frac{3 \mu}{\sqrt{n}}-\frac{\sqrt{n}}{4 \mu}-\frac{1}{12}$,

$a_{3}=\frac{3648 \mu^{6}-16 \mu^{3} n^{3 / 2}(5 n+9)+4 \mu n^{5 / 2}-n^{3}-12 \mu^{2}(n-2) n^{2}+1920 \mu^{5} \sqrt{n}+48 \mu^{4} n(3 n-8)}{64 \mu^{3} n^{3 / 2}}$,

$a_{4}=\frac{542208 \mu^{8}-192 \mu^{5} n^{3 / 2}(59 n+200)+48 \mu^{3} n^{5 / 2}(15 n+26)-12 \mu n^{7 / 2}+3 n^{4}+8 \mu^{4} n^{3}(13 n-1050)+24 \mu^{2} n^{3}(3 n-7)+306432 \mu^{7} \sqrt{n}+192 \mu^{6} n(121 n-327)}{768 \mu^{4} n^{2}}$

$+\frac{3121}{480}, \quad a_{5}=-1, \quad a_{6}=-\frac{3 \mu}{\sqrt{n}}+\frac{\sqrt{n}}{4 \mu}-2, a_{7}=\frac{1}{16}\left(-\frac{480 \mu^{2}}{n}+\left(20-\frac{1}{\mu^{2}}\right) n-\frac{192 \mu}{\sqrt{n}}+\frac{8 \sqrt{n}}{\mu}+4\right)$,

$a_{8}=-\frac{399 \mu^{3}}{n^{3 / 2}}+\frac{n^{3 / 2}}{64 \mu^{3}}-\frac{180 \mu^{2}}{n}-\frac{n}{8 \mu^{2}}+\frac{\mu(59 n+120)}{4 \sqrt{n}}-\frac{3 \sqrt{n}(5 n+6)}{16 \mu}+15 n+9, a_{10}=1, \quad a_{11}=\frac{6 \mu}{\sqrt{n}}-\frac{\sqrt{n}}{4 \mu}+3$,

$a_{12}=\frac{1}{16}\left(\frac{1440 \mu^{2}}{n}+\left(\frac{1}{\mu^{2}}-120\right) n+\frac{480 \mu}{\sqrt{n}}-\frac{12 \sqrt{n}}{\mu}-8\right), a_{14}=-1, \quad a_{15}=\frac{n-40 \mu^{2}}{4 \mu \sqrt{n}}-4, \quad a_{17}=1$. 
Therefore,

$$
\begin{aligned}
& h\left(u_{1}, u_{2}\right)=u_{1}^{4} u_{2}\left(-\frac{399 \mu^{3}}{n^{3 / 2}}+\frac{n^{3 / 2}}{64 \mu^{3}}-\frac{180 \mu^{2}}{n}-\frac{n}{8 \mu^{2}}+\frac{\mu(59 n+120)}{4 \sqrt{n}}-\frac{3 \sqrt{n}(5 n+6)}{16 \mu}+15 n+9\right) \\
& +\frac{u_{1}^{4}\left(3648 \mu^{6}-16 \mu^{3} n^{3 / 2}(5 n+9)+4 \mu n^{5 / 2}-n^{3}-12 \mu^{2}(n-2) n^{2}+1920 \mu^{5} \sqrt{n}+48 \mu^{4} n(3 n-8)\right)}{64 \mu^{3} n^{3 / 2}} \\
& +u_{1}^{5}\left(\frac{542208 \mu^{8}-192 \mu^{5} n^{3 / 2}(59 n+200)+48 \mu^{3} n^{5 / 2}(15 n+26)-12 \mu n^{7 / 2}+3 n^{4}+8 \mu^{4} n^{3}(13 n-1050)+24 \mu^{2} n^{3}(3 n-7)+306432 \mu^{7} \sqrt{n}+192 \mu^{6} n(121 n-327)}{768 \mu^{4} n^{2}}+\frac{3121}{480}\right) \\
& +u_{1}^{3}\left(\frac{6 \mu^{2}}{n}+\frac{1}{16}\left(\frac{1}{\mu^{2}}+4\right) n+\frac{3 \mu}{\sqrt{n}}-\frac{\sqrt{n}}{4 \mu}-\frac{1}{12}\right)+\frac{1}{16} u_{1}^{3} u_{2}^{2}\left(\frac{1440 \mu^{2}}{n}+\left(\frac{1}{\mu^{2}}-120\right) n+\frac{480 \mu}{\sqrt{n}}-\frac{12 \sqrt{n}}{\mu}-8\right) \\
& +\frac{1}{16} u_{1}^{3} u_{2}\left(-\frac{480 \mu^{2}}{n}+\left(20-\frac{1}{\mu^{2}}\right) n-\frac{192 \mu}{\sqrt{n}}+\frac{8 \sqrt{n}}{\mu}+4\right)+u_{1}^{2}\left(\frac{\mu}{\sqrt{n}}-\frac{\sqrt{n}}{4 \mu}+1\right)+u_{1}^{2} u_{2}^{3}\left(\frac{n-40 \mu^{2}}{4 \mu \sqrt{n}}-4\right) \\
& +u_{1}^{2} u_{2}^{2}\left(\frac{6 \mu}{\sqrt{n}}-\frac{\sqrt{n}}{4 \mu}+3\right)+u_{1}^{2} u_{2}\left(-\frac{3 \mu}{\sqrt{n}}+\frac{\sqrt{n}}{4 \mu}-2\right)+u_{1} u_{2}^{4}-u_{1} u_{2}^{3}+u_{1} u_{2}^{2}-u_{1} u_{2} .
\end{aligned}
$$

Finally, the center manifold can be expressed as

$$
\begin{aligned}
& \theta=-\frac{23134208 T^{5} \mu^{9}}{n^{9 / 2}}+\frac{57835520 T^{4} \mu^{9}}{n^{9 / 2}}-\frac{57835520 T^{3} \mu^{9}}{n^{9 / 2}}+\frac{28917760 T^{2} \mu^{9}}{n^{9 / 2}}-\frac{7229440 T \mu^{9}}{n^{9 / 2}}+\frac{722944 \mu^{9}}{n^{9 / 2}} \\
& -\frac{991232 T^{5} \mu^{7}}{n^{5 / 2}}+\frac{5627904 T^{5} \mu^{7}}{n^{7 / 2}}-\frac{1634304 Q T^{4} \mu^{7}}{n^{7 / 2}}+\frac{2478080 T^{4} \mu^{7}}{n^{5 / 2}}-\frac{12201984 T^{4} \mu^{7}}{n^{7 / 2}}+\frac{3268608 Q T^{3} \mu^{7}}{n^{7 / 2}} \\
& -\frac{2478080 T^{3} \mu^{7}}{n^{5 / 2}}+\frac{10334208 T^{3} \mu^{7}}{n^{7 / 2}}-\frac{2451456 Q T^{2} \mu^{7}}{n^{7 / 2}}+\frac{1239040 T^{2} \mu^{7}}{n^{5 / 2}}-\frac{4233216 T^{2} \mu^{7}}{n^{7 / 2}}-\frac{102144 Q \mu^{7}}{n^{7 / 2}} \\
& +\frac{817152 Q T \mu^{7}}{n^{7 / 2}}-\frac{309760 T \mu^{7}}{n^{5 / 2}}+\frac{824832 T \mu^{7}}{n^{7 / 2}}+\frac{30976 \mu^{7}}{n^{5 / 2}}-\frac{59136 \mu^{7}}{n^{7 / 2}}-\frac{13312 T^{5} \mu^{5}}{3 \sqrt{n}}+\frac{112640 T^{5} \mu^{5}}{n^{3 / 2}} \\
& -\frac{5899264 T^{5} \mu^{5}}{15 n^{5 / 2}}+\frac{60416 Q T^{4} \mu^{5}}{n^{3 / 2}}+\frac{245760 Q T^{4} \mu^{5}}{n^{5 / 2}}+\frac{33280 T^{4} \mu^{5}}{3 \sqrt{n}}-\frac{332800 T^{4} \mu^{5}}{n^{3 / 2}}+\frac{2064896 T^{4} \mu^{5}}{3 n^{5 / 2}} \\
& -\frac{46080 Q^{2} T^{3} \mu^{5}}{n^{5 / 2}}-\frac{120832 Q T^{3} \mu^{5}}{n^{3 / 2}}-\frac{384000 Q T^{3} \mu^{5}}{n^{5 / 2}}-\frac{33280 T^{3} \mu^{5}}{3 \sqrt{n}}+\frac{384000 T^{3} \mu^{5}}{n^{3 / 2}}-\frac{1373696 T^{3} \mu^{5}}{3 n^{5 / 2}} \\
& +\frac{5760 Q^{2} \mu^{5}}{n^{5 / 2}}+\frac{69120 Q^{2} T^{2} \mu^{5}}{n^{5 / 2}}+\frac{90624 Q T^{2} \mu^{5}}{n^{3 / 2}}+\frac{207360 Q T^{2} \mu^{5}}{n^{5 / 2}}+\frac{16640 T^{2} \mu^{5}}{3 \sqrt{n}}-\frac{217600 T^{2} \mu^{5}}{n^{3 / 2}}+\frac{438016 T^{2} \mu^{5}}{3 n^{5 / 2}} \\
& +\frac{3776 Q \mu^{5}}{n^{3 / 2}}+\frac{1920 Q \mu^{5}}{n^{5 / 2}}-\frac{34560 Q^{2} T \mu^{5}}{n^{5 / 2}}-\frac{30208 Q T \mu^{5}}{n^{3 / 2}}-\frac{42240 Q T \mu^{5}}{n^{5 / 2}}-\frac{4160 T \mu^{5}}{3 \sqrt{n}}+\frac{60800 T \mu^{5}}{n^{3 / 2}} \\
& -\frac{71488 T \mu^{5}}{3 n^{5 / 2}}+\frac{416 \mu^{5}}{3 \sqrt{n}}-\frac{6720 \mu^{5}}{n^{3 / 2}}+\frac{28832 \mu^{5}}{15 n^{5 / 2}}-\frac{3072 T^{5} \mu^{3}}{\sqrt{n}}+\frac{9216 T^{5} \mu^{3}}{n^{3 / 2}}-\frac{3840 Q T^{4} \mu^{3}}{\sqrt{n}}-\frac{7680 Q T^{4} \mu^{3}}{n^{3 / 2}} \\
& +\frac{10752 T^{4} \mu^{3}}{\sqrt{n}}-\frac{12800 T^{4} \mu^{3}}{n^{3 / 2}}-\frac{160 Q^{3} \mu^{3}}{n^{3 / 2}}+\frac{3840 Q^{2} T^{3} \mu^{3}}{\sqrt{n}}+\frac{3328 Q^{2} T^{3} \mu^{3}}{n^{3 / 2}}-\frac{640 Q T^{3} \mu^{3}}{\sqrt{n}}+\frac{7040 Q T^{3} \mu^{3}}{n^{3 / 2}} \\
& -\frac{9472 T^{3} \mu^{3}}{\sqrt{n}}+\frac{24320 T^{3} \mu^{3}}{3 n^{3 / 2}}-\frac{480 Q^{2} \mu^{3}}{\sqrt{n}}+\frac{160 Q^{2} \mu^{3}}{n^{3 / 2}}-\frac{640 Q^{3} T^{2} \mu^{3}}{n^{3 / 2}}-\frac{5760 Q^{2} T^{2} \mu^{3}}{\sqrt{n}}-\frac{2688 Q^{2} T^{2} \mu^{3}}{n^{3 / 2}} \\
& +\frac{6720 Q T^{2} \mu^{3}}{\sqrt{n}}-\frac{1920 Q T^{2} \mu^{3}}{n^{3 / 2}}+\frac{1920 T^{2} \mu^{3}}{\sqrt{n}}-\frac{3200 T^{2} \mu^{3}}{n^{3 / 2}}+\frac{800 Q \mu^{3}}{\sqrt{n}}-\frac{160 Q \mu^{3}}{n^{3 / 2}}+\frac{640 Q^{3} T \mu^{3}}{n^{3 / 2}}+\frac{2880 Q^{2} T \mu^{3}}{\sqrt{n}} \\
& +\frac{192 Q^{2} T \mu^{3}}{n^{3 / 2}}-\frac{4320 Q T \mu^{3}}{\sqrt{n}}+\frac{480 Q T \mu^{3}}{n^{3 / 2}}+\frac{768 T \mu^{3}}{\sqrt{n}}+\frac{640 T \mu^{3}}{n^{3 / 2}}-\frac{256 \mu^{3}}{\sqrt{n}}-\frac{64 \mu^{3}}{3 n^{3 / 2}}-\frac{128 T^{5} \mu}{\sqrt{n}}+\frac{4 Q^{4} \mu}{\sqrt{n}} \\
& +\frac{64 Q T^{4} \mu}{\sqrt{n}}+\frac{192 T^{4} \mu}{\sqrt{n}}-\frac{16 Q^{3} \mu}{\sqrt{n}}-\frac{32 Q^{2} T^{3} \mu}{\sqrt{n}}-\frac{32 Q T^{3} \mu}{\sqrt{n}}-\frac{160 T^{3} \mu}{\sqrt{n}}+\frac{28 Q^{2} \mu}{\sqrt{n}}+\frac{16 Q^{3} T^{2} \mu}{\sqrt{n}}-\frac{16 Q^{2} T^{2} \mu}{\sqrt{n}} \\
& +\frac{48 Q T^{2} \mu}{\sqrt{n}}+\frac{48 T^{2} \mu}{\sqrt{n}}-\frac{24 Q \mu}{\sqrt{n}}-\frac{8 Q^{4} T \mu}{\sqrt{n}}+\frac{24 Q^{3} T \mu}{\sqrt{n}}-\frac{40 Q^{2} T \mu}{\sqrt{n}}+\frac{24 Q T \mu}{\sqrt{n}}-\frac{24 T \mu}{\sqrt{n}}+\frac{12 \mu}{\sqrt{n}} .
\end{aligned}
$$


The dynamics on the center manifold is given by

$$
\begin{aligned}
& \frac{d u_{1}}{d N}=u_{1}^{4}\left(\frac{3 \mu\left(-13 \mu^{2}-6 \mu \sqrt{n}+n\right)}{n^{3 / 2}}+u_{2}\left(\frac{3 \mu\left(65 \mu^{2}+24 \mu \sqrt{n}-n(4 n+3)\right)}{n^{3 / 2}}-\frac{3}{2}\right)+\frac{3}{4}\right) \\
& +\frac{3 u_{1}^{5}\left(-504 \mu^{4}+4 \mu n^{3 / 2}(4 n+5)-3 n^{2}-260 \mu^{3} \sqrt{n}+\mu^{2}(53-12 n) n\right)}{4 n^{2}} \\
& +u_{1}^{3}\left(-\frac{3 \mu(2 \mu+\sqrt{n})}{n}+u_{2}^{2}\left(\frac{3}{4}-\frac{9 \mu(4 \mu+\sqrt{n})}{n}\right)\right. \\
& \left.+u_{2}\left(\frac{6 \mu(3 \mu+\sqrt{n})}{n}-\frac{3}{4}\right)+\frac{3}{4}\right)+u_{1}^{2}\left(-\frac{3 \mu}{\sqrt{n}}+\frac{3 \mu u_{2}^{3}}{\sqrt{n}}-\frac{3 \mu u_{2}^{2}}{\sqrt{n}}+\frac{3 \mu u_{2}}{\sqrt{n}}\right) \\
& \frac{d u_{2}}{d N}=u_{1}^{4}\left(\frac{3}{4}\left(-\frac{52 \mu^{3}}{n^{3 / 2}}+\frac{n^{3 / 2}}{\mu}-\frac{24 \mu^{2}}{n}+\frac{\mu(4-8 n)}{\sqrt{n}}-6 n+1\right)\right. \\
& +u_{2}\left(\frac{195 \mu^{3}}{\left.\left.n^{3 / 2}-\frac{9 n^{3 / 2}}{4 \mu}+\frac{3 n^{2}}{16 \mu^{2}}+\frac{3 \mu^{2}(13 n+24)}{n}+\frac{3 \mu(10 n-3)}{\sqrt{n}}+12 n-\frac{3}{2}\right)\right)}\right. \\
& +u_{1}^{3}\left(u_{2}^{2}\left(\frac{9 n^{3 / 2}}{8 \mu}-\frac{36 \mu^{2}}{n}-\frac{3 \mu(7 n+3)}{\sqrt{n}}-18 n+\frac{3}{4}\right)\right. \\
& \left.+\frac{3}{4} u_{2}\left(-\frac{n^{3 / 2}}{\mu}+\frac{24 \mu^{2}}{n}+\frac{8 \mu(n+1)}{\sqrt{n}}+12 n-1\right)-\frac{3\left(2 \mu^{2}+n^{2}+\mu \sqrt{n}\right)}{n}+\frac{3}{4}\right) \\
& +u_{1}^{5}\left(\frac{-6048 \mu^{6}+48 \mu^{3}(5-3 n) n^{3 / 2}+18 \mu n^{7 / 2}-3 n^{4}-3120 \mu^{5} \sqrt{n}+12 \mu^{4}(53-64 n) n}{\sqrt{n}}-\frac{9}{4}\right)
\end{aligned}
$$

For which the origin is unstable (see Fig. 12). Using the Theorem 2, we conclude that the center manifold of origin for the system (242), (243), and the origin are unstable (saddle).

Proof Part (ii) More general, by introducing the linear transformation

$$
\begin{aligned}
& \left(u_{1}, u_{2}, v\right) \\
& =\left(\frac{\mu(4-8 T)}{\sqrt{n}}, \frac{\mu(4-8 T)}{\sqrt{n}}+Q-Q^{*}, \theta Q^{*}+\frac{4 \mu(2 T-1)}{\sqrt{n}}\right),
\end{aligned}
$$

and taking Taylor series near $\left(u_{1}, u_{2}, v\right)=(0,0,0)$, and taking Taylor series near $\left(u_{1}, u_{2}, v\right)=(0,0,0)$ up to fifth order we obtain the system (233), (234), (235) can be written into its Jordan canonical form:

$$
\left(\begin{array}{c}
\frac{d u_{1}}{d N} \\
\frac{d u_{2}}{d N} \\
\frac{d v}{d N}
\end{array}\right)=\left(\begin{array}{ccc}
0 & 0 & 0 \\
0 & 0 & 0 \\
0 & 0 & -\frac{3}{2} Q^{*}
\end{array}\right)\left(\begin{array}{c}
u_{1} \\
u_{2} \\
v
\end{array}\right)+\left(\begin{array}{c}
f_{1}^{*}(\mathbf{u}, v) \\
f_{2}^{*}(\mathbf{u}, v) \\
g^{*}(\mathbf{u}, v)
\end{array}\right)
$$
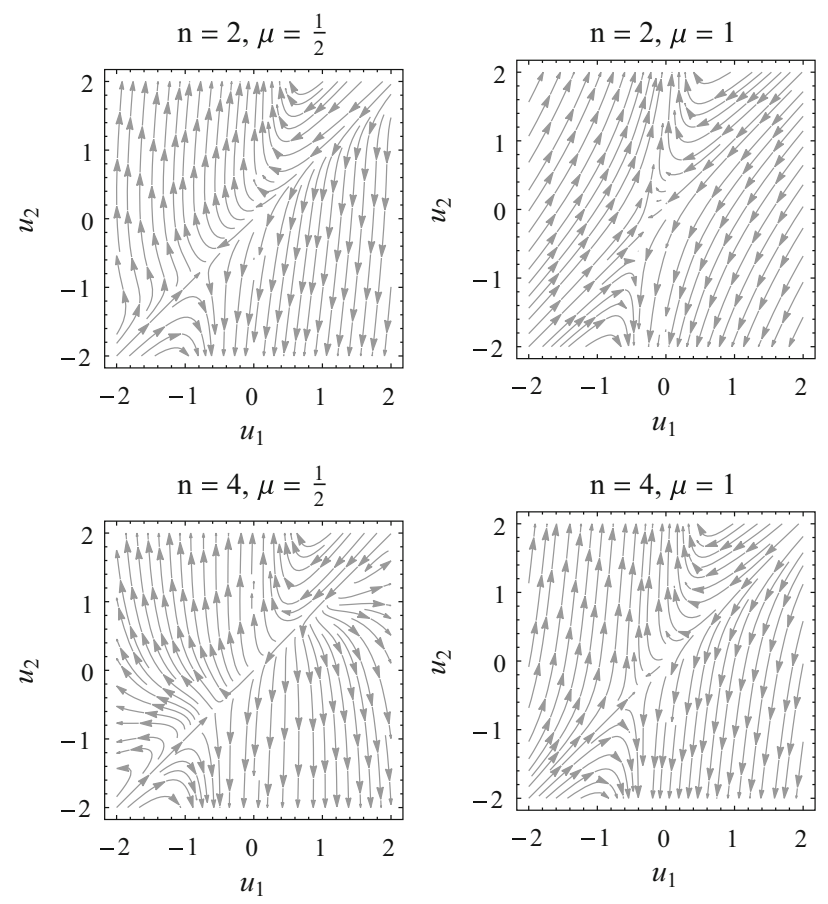

Fig. 12 Numerical solutions of the system (280) shows the instability of the origin $\left(u_{1}, u_{2}\right)=(0,0)$ 
where

$f_{1}^{*}(\mathbf{u}, v)=\frac{\left(u_{1}+v\right)^{2}\left(3 n^{3 / 2} Q^{* 3} u_{1}^{3}+12 \mu n Q^{* 2} u_{1}^{2}\left(Q^{*}-u_{1}+u_{2}\right)-32 \mu^{3}\left(Q^{*}-u_{1}+u_{2}\right)\left(6 Q^{* 2}-(3 n-1)\left(u_{1}+v\right)^{2}\right)+8 \mu^{2} \sqrt{n} Q^{*} u_{1}\left((3 n-1)\left(u_{1}+v\right)^{2}-6 Q^{* 2}+6 Q^{*}\left(u_{1}-u_{2}\right)\right)\right)}{64 \mu^{2} \sqrt{n} Q^{* 4}}$

$f_{2}^{*}(\mathbf{u}, v)=-\frac{3 \sqrt{n} \mu u_{1}^{5}}{2 Q^{* 4}}+\frac{\mu u_{1}^{5}}{2 \sqrt{n} Q^{* 4}}-\frac{3 n^{5 / 2} u_{1}^{5}}{16 Q^{* 2} \mu}+\frac{7 n^{3 / 2} u_{1}^{5}}{16 Q^{* 2} \mu}-\frac{3 \sqrt{n} u_{1}^{5}}{16 Q^{* 2} \mu}+\frac{3 n^{5 / 2} u_{1}^{5}}{16 Q^{* 4} \mu}-\frac{n^{3 / 2} u_{1}^{5}}{16 Q^{* 4} \mu}+\frac{3 n u_{1}^{5}}{64 Q^{*} \mu^{2}}+\frac{3 n^{2} u_{1}^{5}}{2 Q^{* 3}}-\frac{n u_{1}^{5}}{8 Q^{* 3}}-\frac{u_{1}^{5}}{8 Q^{* 3}}-\frac{3 n^{2} u_{2} u_{1}^{4}}{2 Q^{* 3}}+\frac{n u_{2} u_{1}^{4}}{2 Q^{* 3}}+\frac{6 n^{2} v u_{1}^{4}}{Q^{* 3}}$

$-\frac{n v u_{1}^{4}}{2 Q^{* 3}}-\frac{v u_{1}^{4}}{2 Q^{* 3}}+\frac{3 \sqrt{n} u_{2} \mu u_{1}^{4}}{2 Q^{* 4}}-\frac{u_{2} \mu u_{1}^{4}}{2 \sqrt{n} Q^{* 4}}-\frac{6 \sqrt{n} v \mu u_{1}^{4}}{Q^{* 4}}+\frac{2 v \mu u_{1}^{4}}{\sqrt{n} Q^{* 4}}+\frac{3 \sqrt{n} \mu u_{1}^{4}}{2 Q^{* 3}}-\frac{\mu u_{1}^{4}}{2 \sqrt{n} Q^{* 3}}-\frac{3 n^{3 / 2} u_{2} u_{1}^{4}}{4 Q^{* 2} \mu}+\frac{3 \sqrt{n} u_{2} u_{1}^{4}}{16 Q^{* 2} \mu}-\frac{3 n^{5 / 2} v u_{1}^{4}}{4 Q^{* 2} \mu}+\frac{n^{3 / 2} v u_{1}^{4}}{Q^{* 2} \mu}-\frac{3 \sqrt{n} v u_{1}^{4}}{8 Q^{* 2} \mu}+\frac{3 n^{5 / 2} v u_{1}^{4}}{4 Q^{* 4} \mu}$

$-\frac{n^{3 / 2} v u_{1}^{4}}{4 Q^{* 4} \mu}-\frac{3 n^{3 / 2} u_{1}^{4}}{4 Q^{*} \mu}+\frac{3 \sqrt{n} u_{1}^{4}}{16 Q^{*} \mu}-\frac{3 n^{2} u_{1}^{4}}{4 Q^{* 2}}+\frac{7 n u_{1}^{4}}{4 Q^{* 2}}+\frac{3 u_{1}^{4}}{4 Q^{* 2}}+\frac{3 n v u_{1}^{4}}{32 Q^{*} \mu^{2}}+\frac{3 n^{2} u_{1}^{4}}{4 Q^{* 4}}-\frac{n u_{1}^{4}}{4 Q^{* 4}}+\frac{9 n^{2} v^{2} u_{1}^{3}}{Q^{* 3}}-\frac{3 n v^{2} u_{1}^{3}}{4 Q^{* 3}}-\frac{3 v^{2} u_{1}^{3}}{4 Q^{* 3}}-\frac{3 n u_{2} u_{1}^{3}}{Q^{* 2}}-\frac{3 u_{2} u_{1}^{3}}{4 Q^{* 2}}-\frac{6 n^{2} u_{2} v u_{1}^{3}}{Q^{* 3}}+\frac{2 n u_{2} v u_{1}^{3}}{Q^{* 3}}-\frac{3 n^{2} v u_{1}^{3}}{Q^{* 2}}$

$+\frac{4 n v u_{1}^{3}}{Q^{* 2}}+\frac{3 v u_{1}^{3}}{2 Q^{* 2}}+\frac{3 n^{2} v u_{1}^{3}}{Q^{* 4}}-\frac{n v u_{1}^{3}}{Q^{* 4}}-\frac{9 \sqrt{n} v^{2} \mu u_{1}^{3}}{Q^{* 4}}+\frac{3 v^{2} \mu u_{1}^{3}}{\sqrt{n} Q^{* 4}}+\frac{6 \sqrt{n} u_{2} v \mu u_{1}^{3}}{Q^{* 4}}-\frac{2 u_{2} v \mu u_{1}^{3}}{\sqrt{n} Q^{* 4}}+\frac{6 \sqrt{n} v \mu u_{1}^{3}}{Q^{* 3}}-\frac{2 v \mu u_{1}^{3}}{\sqrt{n} Q^{* 3}}+\frac{3 \mu u_{1}^{3}}{\sqrt{n} Q^{* 2}}-\frac{3 n u_{1}^{3}}{Q^{*}}-\frac{3 u_{1}^{3}}{4 Q^{*}}+\frac{3 n^{3 / 2} u_{2}^{2} u_{1}^{3}}{8 Q^{* 2} \mu}-\frac{9 n^{5 / 2} v^{2} u_{1}^{3}}{8 Q^{* 2} \mu}$

$+\frac{3 n^{3 / 2} v^{2} u_{1}^{3}}{4 Q^{* 2} \mu}-\frac{3 \sqrt{n} v^{2} u_{1}^{3}}{16 Q^{* 2} \mu}+\frac{9 n^{5 / 2} v^{2} u_{1}^{3}}{8 Q^{* 4} \mu}-\frac{3 n^{3 / 2} v^{2} u_{1}^{3}}{8 Q^{* 4} \mu}+\frac{3 n^{3 / 2} u_{1}^{3}}{8 \mu}+\frac{3 n^{3 / 2} u_{2} u_{1}^{3}}{4 Q^{*} \mu}-\frac{3 n^{3 / 2} u_{2} v u_{1}^{3}}{2 Q^{* 2} \mu}+\frac{3 \sqrt{n} u_{2} v u_{1}^{3}}{8 Q^{* 2} \mu}-\frac{3 n^{3 / 2} v u_{1}^{3}}{2 Q^{*} \mu}+\frac{3 \sqrt{n} v u_{1}^{3}}{8 Q^{*} \mu}-\frac{3 n^{3 / 2} u_{1}^{3}}{8 Q^{* 2} \mu}+\frac{3 n v^{2} u_{1}^{3}}{64 Q^{*} \mu^{2}}+\frac{6 n^{2} v^{3} u_{1}^{2}}{Q^{* 3}}$

$-\frac{n v^{3} u_{1}^{2}}{2 Q^{* 3}}-\frac{v^{3} u_{1}^{2}}{2 Q^{* 3}}+\frac{3 n u_{2}^{2} u_{1}^{2}}{2 Q^{* 2}}-\frac{9 n^{2} u_{2} v^{2} u_{1}^{2}}{Q^{* 3}}+\frac{3 n u_{2} v^{2} u_{1}^{2}}{Q^{* 3}}-\frac{9 n^{2} v^{2} u_{1}^{2}}{2 Q^{* 2}}+\frac{3 n v^{2} u_{1}^{2}}{Q^{* 2}}+\frac{3 v^{2} u_{1}^{2}}{4 Q^{* 2}}+\frac{9 n^{2} v^{2} u_{1}^{2}}{2 Q^{* 4}}-\frac{3 n v^{2} u_{1}^{2}}{2 Q^{* 4}}+\frac{3 n u_{1}^{2}}{2}+\frac{3 n u_{2} u_{1}^{2}}{Q^{*}}-\frac{6 n u_{2} v u_{1}^{2}}{Q^{* 2}}-\frac{3 u_{2} v u_{1}^{2}}{2 Q^{* 2}}-\frac{6 n v u_{1}^{2}}{Q^{*}}-\frac{3 v u_{1}^{2}}{2 Q^{*}}$

$-\frac{6 \sqrt{n} v^{3} \mu u_{1}^{2}}{Q^{* 4}}+\frac{2 v^{3} \mu u_{1}^{2}}{\sqrt{n} Q^{* 4}}+\frac{9 \sqrt{n} u_{2} v^{2} \mu u_{1}^{2}}{Q^{* 4}}-\frac{3 u_{2} v^{2} \mu u_{1}^{2}}{\sqrt{n} Q^{* 4}}+\frac{9 \sqrt{n} v^{2} \mu u_{1}^{2}}{Q^{* 3}}-\frac{3 v^{2} \mu u_{1}^{2}}{\sqrt{n} Q^{* 3}}-\frac{3 u_{2} \mu u_{1}^{2}}{\sqrt{n} Q^{* 2}}+\frac{6 v \mu u_{1}^{2}}{\sqrt{n} Q^{* 2}}-\frac{3 \mu u_{1}^{2}}{\sqrt{n} Q^{*}}-\frac{3 n^{5 / 2} v^{3} u_{1}^{2}}{4 Q^{* 2} \mu}+\frac{n^{3 / 2} v^{3} u_{1}^{2}}{4 Q^{* 2} \mu}+\frac{3 n^{5 / 2} v^{3} u_{1}^{2}}{4 Q^{* 4} \mu}-\frac{n^{3 / 2} v^{3} u_{1}^{2}}{4 Q^{* 4} \mu}$

$-\frac{3 n^{3 / 2} u_{2} v^{2} u_{1}^{2}}{4 Q^{* 2} \mu}+\frac{3 \sqrt{n} u_{2} v^{2} u_{1}^{2}}{16 Q^{* 2} \mu}-\frac{3 n^{3 / 2} v^{2} u_{1}^{2}}{4 Q^{*} \mu}+\frac{3 \sqrt{n} v^{2} u_{1}^{2}}{16 Q^{*} \mu}+\frac{3 n^{3 / 2} u_{2}^{2} v u_{1}^{2}}{4 Q^{* 2} \mu}+\frac{3 n^{3 / 2} v u_{1}^{2}}{4 \mu}+\frac{3 n^{3 / 2} u_{2} v u_{1}^{2}}{2 Q^{*} \mu}-\frac{3 n^{3 / 2} v u_{1}^{2}}{4 Q^{* 2} \mu}-\frac{3 n u_{1}^{2}}{2 Q^{* 2}}+\frac{3 n^{2} v^{4} u_{1}}{2 Q^{* 3}}-\frac{n v^{4} u_{1}}{8 Q^{* 3}}-\frac{v^{4} u_{1}}{8 Q^{* 3}}-\frac{6 n^{2} u_{2} v^{3} u_{1}}{Q^{* 3}}$

$+\frac{2 n u_{2} v^{3} u_{1}}{Q^{* 3}}-\frac{3 n^{2} v^{3} u_{1}}{Q^{* 2}}+\frac{n v^{3} u_{1}}{Q^{* 2}}+\frac{3 n^{2} v^{3} u_{1}}{Q^{* 4}}-\frac{n v^{3} u_{1}}{Q^{* 4}}-\frac{3 n u_{2} v^{2} u_{1}}{Q^{* 2}}-\frac{3 u_{2} v^{2} u_{1}}{4 Q^{* 2}}-\frac{3 n v^{2} u_{1}}{Q^{*}}-\frac{3 v^{2} u_{1}}{4 Q^{*}}+\frac{3 n u_{2}^{2} v u_{1}}{Q^{* 2}}+3 n v u_{1}+\frac{6 n u_{2} v u_{1}}{Q^{*}}-\frac{3 n v u_{1}}{Q^{* 2}}-\frac{3 \sqrt{n} v^{4} \mu u_{1}}{2 Q^{* 4}}+\frac{v^{4} \mu u_{1}}{2 \sqrt{n} Q^{* 4}}$

$+\frac{6 \sqrt{n} u_{2} v^{3} \mu u_{1}}{Q^{* 4}}-\frac{2 u_{2} v^{3} \mu u_{1}}{\sqrt{n} Q^{* 4}}+\frac{6 \sqrt{n} v^{3} \mu u_{1}}{Q^{* 3}}-\frac{2 v^{3} \mu u_{1}}{\sqrt{n} Q^{* 3}}+\frac{3 v^{2} \mu u_{1}}{\sqrt{n} Q^{* 2}}-\frac{6 u_{2} v \mu u_{1}}{\sqrt{n} Q^{* 2}}-\frac{6 v \mu u_{1}}{\sqrt{n} Q^{*}}-\frac{3 n^{5 / 2} v^{4} u_{1}}{16 Q^{* 2} \mu}+\frac{n^{3 / 2} v^{4} u_{1}}{16 Q^{* 2} \mu}+\frac{3 n^{5 / 2} v^{4} u_{1}}{16 Q^{* 4} \mu}-\frac{n^{3 / 2} v^{4} u_{1}}{16 Q^{* 4} \mu}-\frac{6 v \mu u_{1}}{\sqrt{n} Q^{*}}+\frac{3 n^{3 / 2} u_{2}^{2} v^{2} u_{1}}{8 Q^{* 2} \mu}$

$+\frac{3 n^{3 / 2} v^{2} u_{1}}{8 \mu}+\frac{3 n^{3 / 2} u_{2} v^{2} u_{1}}{4 Q^{*} \mu}-\frac{3 n^{3 / 2} v^{2} u_{1}}{8 Q^{* 2} \mu}-\frac{3 n^{2} u_{2} v^{4}}{2 Q^{* 3}}+\frac{n u_{2} v^{4}}{2 Q^{* 3}}-\frac{3 n^{2} v^{4}}{4 Q^{* 2}}+\frac{n v^{4}}{4 Q^{* 2}}+\frac{3 n^{2} v^{4}}{4 Q^{* 4}}-\frac{n v^{4}}{4 Q^{* 4}}+\frac{3 n u_{2}^{2} v^{2}}{2 Q^{* 2}}+\frac{3 n v^{2}}{2}+\frac{3 n u_{2} v^{2}}{Q^{*}}-\frac{3 n v^{2}}{2 Q^{* 2}}+\frac{3 \sqrt{n} u_{2} v^{4} \mu}{2 Q^{* 4}}-\frac{u_{2} v^{4} \mu}{2 \sqrt{n} Q^{* 4}}$

$+\frac{3 \sqrt{n} v^{4} \mu}{2 Q^{* 3}}-\frac{v^{4} \mu}{2 \sqrt{n} Q^{* 3}}-\frac{3 u_{2} v^{2} \mu}{\sqrt{n} Q^{* 2}}-\frac{3 v^{2} \mu}{\sqrt{n} Q^{*}}$,

$g^{*}(\mathbf{u}, v)=\frac{3 \sqrt{n} \mu u_{1}^{5}}{2 Q^{* 4}}-\frac{\mu u_{1}^{5}}{2 \sqrt{n} Q^{* 4}}-\frac{3 n^{3 / 2} u_{1}^{5}}{16 Q^{* 2} \mu}+\frac{\sqrt{n} u_{1}^{5}}{8 Q^{* 2} \mu}-\frac{3 n u_{1}^{5}}{64 Q^{*} \mu^{2}}-\frac{11 n^{2} u_{1}^{5}}{64 Q^{* 3}}-\frac{7 n u_{1}^{5}}{16 Q^{* 3}}+\frac{61 u_{1}^{5}}{320 Q^{* 3}}-\frac{15 n^{2} v u_{1}^{4}}{16 Q^{* 3}}-\frac{7 n v u_{1}^{4}}{4 Q^{* 3}}+\frac{13 v u_{1}^{4}}{16 Q^{* 3}}-\frac{3 \sqrt{n} u_{2} \mu u_{1}^{4}}{2 Q^{* 4}}+\frac{u_{2} \mu u_{1}^{4}}{2 \sqrt{n} Q^{* 4}}+\frac{6 \sqrt{n} v \mu u_{1}^{4}}{Q^{* 4}}$

$-\frac{2 v \mu u_{1}^{4}}{\sqrt{n} Q^{* 4}}-\frac{9 \sqrt{n} \mu u_{1}^{4}}{8 Q^{* 3}}+\frac{\mu u_{1}^{4}}{4 \sqrt{n} Q^{* 3}}+\frac{3 n^{3 / 2} u_{2} u_{1}^{4}}{16 Q^{* 2} \mu}-\frac{\sqrt{n} u_{2} u_{1}^{4}}{8 Q^{* 2} \mu}-\frac{9 n^{3 / 2} v u_{1}^{4}}{16 Q^{* 2} \mu}+\frac{3 \sqrt{n} v u_{1}^{4}}{16 Q^{* 2} \mu}+\frac{3 n^{3 / 2} u_{1}^{4}}{16 Q^{*} \mu}-\frac{\sqrt{n} u_{1}^{4}}{8 Q^{*} \mu}-\frac{3 n u_{1}^{4}}{4 Q^{* 2}}-\frac{u_{1}^{4}}{Q^{* 2}}-\frac{3 n v u_{1}^{4}}{32 Q^{*} \mu^{2}}-\frac{65 n^{2} v^{2} u_{1}^{3}}{32 Q^{* 3}}-\frac{21 n v^{2} u_{1}^{3}}{8 Q^{* 3}}+\frac{43 v^{2} u_{1}^{3}}{32 Q^{* 3}}$

$+\frac{3 n u_{2} u_{1}^{3}}{4 Q^{* 2}}+\frac{u_{2} u_{1}^{3}}{Q^{* 2}}-\frac{9 n v u_{1}^{3}}{4 Q^{* 2}}-\frac{9 v u_{1}^{3}}{4 Q^{* 2}}+\frac{9 \sqrt{n} v^{2} \mu u_{1}^{3}}{Q^{* 4}}-\frac{3 v^{2} \mu u_{1}^{3}}{\sqrt{n} Q^{* 4}}-\frac{6 \sqrt{n} u_{2} v \mu u_{1}^{3}}{Q^{* 4}}+\frac{2 u_{2} v \mu u_{1}^{3}}{\sqrt{n} Q^{* 4}}-\frac{9 \sqrt{n} v \mu u_{1}^{3}}{2 Q^{* 3}}+\frac{v \mu u_{1}^{3}}{\sqrt{n} Q^{* 3}}-\frac{3 \mu u_{1}^{3}}{\sqrt{n} Q^{* 2}}+\frac{3 n u_{1}^{3}}{8 Q^{*}}+\frac{u_{1}^{3}}{Q^{*}}-\frac{9 n^{3 / 2} v^{2} u_{1}^{3}}{16 Q^{* 2} \mu}+\frac{9 n^{3 / 2} u_{2} v u_{1}^{3}}{16 Q^{* 2} \mu}$

$-\frac{3 \sqrt{n} u_{2} v u_{1}^{3}}{16 Q^{* 2} u}+\frac{9 n^{3 / 2} v u_{1}^{3}}{16 Q^{*} \mu}-\frac{3 \sqrt{n} v u_{1}^{3}}{16 Q^{*} \mu}+\frac{3 \sqrt{n} u_{1}^{3}}{8 \mu}-\frac{3 n v^{2} u_{1}^{3}}{64 Q^{*} \mu^{2}}-\frac{35 n^{2} v^{3} u_{1}^{2}}{16 Q^{* 3}}-\frac{7 n v^{3} u_{1}^{2}}{4 Q^{* 3}}+\frac{17 v^{3} u_{1}^{2}}{16 Q^{* 3}}-\frac{9 n v^{2} u_{1}^{2}}{4 Q^{* 2}}-\frac{3 v^{2} u_{1}^{2}}{2 Q^{* 2}}+\frac{9 n u_{2} v u_{1}^{2}}{4 Q^{* 2}}+\frac{9 u_{2} v u_{1}^{2}}{4 Q^{* 2}}+\frac{3 n v u_{1}^{2}}{2 Q^{*}}+\frac{9 v u_{1}^{2}}{4 Q^{*}}+\frac{6 \sqrt{n} v^{3} \mu u_{1}^{2}}{Q^{* 4}}$

$-\frac{2 v^{3} \mu u_{1}^{2}}{\sqrt{n} Q^{* 4}}-\frac{9 \sqrt{n} u_{2} v^{2} \mu u_{1}^{2}}{Q^{* 4}}+\frac{3 u_{2} v^{2} \mu u_{1}^{2}}{\sqrt{n} Q^{* 4}}-\frac{27 \sqrt{n} v^{2} \mu u_{1}^{2}}{4 Q^{* 3}}+\frac{3 v^{2} \mu u_{1}^{2}}{2 \sqrt{n} Q^{* 3}}+\frac{3 u_{2} \mu u_{1}^{2}}{\sqrt{n} Q^{* 2}}-\frac{6 v \mu u_{1}^{2}}{\sqrt{n} Q^{* 2}}+\frac{3 \mu u_{1}^{2}}{2 \sqrt{n} Q^{*}}-\frac{3 n^{3 / 2} v^{3} u_{1}^{2}}{16 Q^{* 2} \mu}-\frac{\sqrt{n} v^{3} u_{1}^{2}}{16 Q^{* 2} \mu}+\frac{9 n^{3 / 2} u_{2} v^{2} u_{1}^{2}}{16 Q^{* 2} \mu}+\frac{9 n^{3 / 2} v^{2} u_{1}^{2}}{16 Q^{*} \mu}-\frac{3 \sqrt{n} Q^{*} u_{1}^{2}}{8 \mu}$

$-\frac{3 \sqrt{n} u_{2} u_{1}^{2}}{8 \mu}+\frac{3 \sqrt{n} v u_{1}^{2}}{8 \mu}+\frac{3 u_{1}^{2}}{2}-\frac{75 n^{2} v^{4} u_{1}}{64 Q^{* 3}}-\frac{7 n v^{4} u_{1}}{16 Q^{* 3}}+\frac{25 v^{4} u_{1}}{64 Q^{* 3}}-\frac{3 n v^{3} u_{1}}{4 Q^{* 2}}-\frac{v^{3} u_{1}}{4 Q^{* 2}}+\frac{9 n u_{2} v^{2} u_{1}}{4 Q^{* 2}}+\frac{3 u_{2} v^{2} u_{1}}{2 Q^{* 2}}+\frac{15 n v^{2} u_{1}}{8 Q^{*}}+\frac{3 v^{2} u_{1}}{2 Q^{*}}-\frac{3 u_{2} u_{1}}{2}+\frac{3 v u_{1}}{2}+\frac{3 \sqrt{n} v^{4} \mu u_{1}}{2 Q^{* 4}}-\frac{v^{4} \mu u_{1}}{2 \sqrt{n} Q^{* 4}}$

$-\frac{6 \sqrt{n} u_{2} v^{3} \mu u_{1}}{Q^{* 4}}+\frac{2 u_{2} v^{3} \mu u_{1}}{\sqrt{n} Q^{* 4}}-\frac{9 \sqrt{n} v^{3} \mu u_{1}}{2 Q^{* 3}}+\frac{v^{3} \mu u_{1}}{\sqrt{n} Q^{* 3}}-\frac{3 v^{2} \mu u_{1}}{\sqrt{n} Q^{* 2}}+\frac{6 u_{2} v \mu u_{1}}{\sqrt{n} Q^{* 2}}+\frac{3 v \mu u_{1}}{\sqrt{n} Q^{*}}+\frac{3 n^{3 / 2} u_{2} v^{3} u_{1}}{16 Q^{* 2} \mu}+\frac{\sqrt{n} u_{2} v^{3} u_{1}}{16 Q^{* 2} \mu}+\frac{3 n^{3 / 2} v^{3} u_{1}}{16 Q^{*} \mu}+\frac{\sqrt{n} v^{3} u_{1}}{16 Q^{*} \mu}-\frac{3 \sqrt{n} Q^{*} v u_{1}}{8 \mu}$

$-\frac{3 \sqrt{n} u_{2} v u_{1}}{8 \mu}-\frac{n^{2} v^{5}}{4 Q^{* 3}}+\frac{v^{5}}{20 Q^{* 3}}+\frac{3 n u_{2} v^{3}}{4 Q^{* 2}}+\frac{u_{2} v^{3}}{4 Q^{* 2}}+\frac{3 n v^{3}}{4 Q^{*}}+\frac{v^{3}}{4 Q^{*}}-\frac{3 u_{2} v}{2}-\frac{3 \sqrt{n} u_{2} v^{4} \mu}{2 Q^{* 4}}+\frac{u_{2} v^{4} \mu}{2 \sqrt{n} Q^{* 4}}-\frac{9 \sqrt{n} v^{4} \mu}{8 Q^{* 3}}+\frac{v^{4} \mu}{4 \sqrt{n} Q^{* 3}}+\frac{3 u_{2} v^{2} \mu}{\sqrt{n} Q^{* 2}}+\frac{3 v^{2} \mu}{2 \sqrt{n} Q^{*}}$.

By Theorem 1, exists a 2-dimensional local center manifold of (276), $W^{c}(\mathbf{0})=\left\{(\mathbf{u}, v) \in \mathbb{R}^{2} \times \mathbb{R}: v=h(\mathbf{u})\right\}$, satisfying $\mathbf{h}(\mathbf{0})=0, \quad D h(\mathbf{0})=0, \quad|\mathbf{u}|<\delta$ for $\delta$ sufficiently small. The restriction of (283) to the center manifold is $\frac{d \mathbf{u}}{d N}=\mathbf{f}(\mathbf{u}, h(\mathbf{u}))$, where the function $h(\mathbf{u})$ that satisfies (A.10):

$D h(\mathbf{u}) \cdot \mathbf{f}(\mathbf{u}, h(\mathbf{u}))+\frac{3}{2} Q^{*} h(\mathbf{u})-g(\mathbf{u}, h(\mathbf{u}))=0$. 
Replacing (241) in (284) we find the non-null coefficients

$a_{1}=\frac{\frac{\mu}{\sqrt{n}}+Q^{*}}{Q^{* 2}}-\frac{\sqrt{n}}{4 \mu}, a_{2}=\frac{6 \mu^{2}}{n Q^{* 4}}+\frac{3 \mu}{\sqrt{n} Q^{* 3}}+\frac{1}{16} n\left(\frac{1}{\mu^{2}}+\frac{4\left(5 Q^{* 2}-4\right)}{Q^{* 4}}\right)-\frac{\sqrt{n}}{4 \mu Q^{*}}-\frac{1}{12 Q^{* 2}}$,

$a_{3}=\frac{3648 \mu^{6}+4 \mu n^{5 / 2} Q^{* 5}+16 \mu^{3} n^{3 / 2} Q^{*}\left(3(5 n-3) Q^{* 2}-20 n\right)-n^{3} Q^{* 6}+12 \mu^{2} n^{2} Q^{* 2}\left(n\left(4-5 Q^{* 2}\right)+2 Q^{* 2}\right)+16 \mu^{4} n\left((61 n-24) Q^{* 2}-52 n\right)+1920 \mu^{5} \sqrt{n} Q^{*}}{64 \mu^{3} n^{3 / 2} Q^{* 6}}$,

$a_{4}=-\frac{n^{3 / 2}}{64 \mu^{3} Q^{*}}+\frac{n^{2}}{256 \mu^{4}}+\frac{-201 \mu^{2}+\frac{706 \mu^{4}}{n^{2}}+7 n^{2}}{Q^{* 8}}+\frac{(19-55 n) n^{2}+\mu^{2}(925 n-327)}{4 n Q^{* 6}}+\frac{5\left(3 n^{2}+\mu^{2}(61 n-40)\right)}{4 \mu \sqrt{n} Q^{* 5}}+\frac{399 \mu^{3}-91 \mu n^{2}}{n^{3 / 2} Q^{* 7}}$

$+\frac{5 n\left(\left(661-\frac{36}{\mu^{2}}\right) n-1506\right)+3121}{480 Q^{* 4}}+\frac{(26-45 n) \sqrt{n}}{16 \mu Q^{* 3}}+\frac{(15 n-7) n}{32 \mu^{2} Q^{* 2}}$,

$a_{5}=-\frac{1}{Q^{*}}, a_{6}=\frac{-\frac{12 \mu}{\sqrt{n}}+\frac{\sqrt{n} Q^{* 2}}{\mu}-8 Q^{*}}{4 Q^{* 3}}, a_{7}=-\frac{\frac{480 \mu^{2}}{n}-\frac{8 \sqrt{n} Q^{* 3}}{\mu}+n\left(\frac{Q^{* 4}}{\mu^{2}}+60 Q^{* 2}-80\right)+\frac{192 \mu Q^{*}}{\sqrt{n}}-4 Q^{* 2}}{16 Q^{* 5}}$,

$=\frac{-25536 \mu^{6}-8 \mu n^{5 / 2} Q^{* 5}+192 \mu^{3} n^{3 / 2} Q^{*}\left(3 Q^{* 2}-5 n\left(Q^{* 2}-2\right)\right)+n^{3} Q^{* 6}+12 \mu^{2} n^{2} Q^{* 2}\left(3(5 n-2) Q^{* 2}-20 n\right)+16 \mu^{4} n\left(5(24-61 n) Q^{* 2}+364 n\right)-11520 \mu^{5} \sqrt{n} Q^{*}}{64 \mu^{3} n^{3 / 2} Q^{* 7}}$

$a_{10}=\frac{1}{Q^{* 2}}, \quad a_{11}=\frac{6 \mu}{\sqrt{n} Q^{* 4}}-\frac{\sqrt{n}}{4 \mu Q^{* 2}}+\frac{3}{Q^{* 3}}, a_{12}=\frac{\frac{1440 \mu^{2}}{n}+\frac{n Q^{* 4}}{\mu^{2}}-\frac{12 \sqrt{n} Q^{* 3}}{\mu}+120 n\left(Q^{* 2}-2\right)+\frac{480 \mu Q^{*}}{\sqrt{n}}-8 Q^{* 2}}{16 Q^{* 6}}$,

$a_{14}=-\frac{1}{Q^{* 3}}, \quad a_{15}=\frac{-\frac{40 \mu}{\sqrt{n}}+\frac{\sqrt{n} Q^{* 2}}{\mu}-16 Q^{*}}{4 Q^{* 5}}, \quad a_{17}=\frac{1}{Q^{* 4}}$.

Therefore,

$$
\begin{aligned}
& h\left(u_{1}, u_{2}\right)=\frac{706 \mu^{4} u_{1}^{5}}{n^{2} Q^{* 8}}+\frac{399 \mu^{3} u_{1}^{5}}{n^{3 / 2} Q^{* 7}}-\frac{327 \mu^{2} u_{1}^{5}}{4 n Q^{* 6}}+\frac{925 \mu^{2} u_{1}^{5}}{4 Q^{* 6}} \\
& -\frac{201 \mu^{2} u_{1}^{5}}{Q^{* 8}}+\frac{305 \sqrt{n} \mu u_{1}^{5}}{4 Q^{* 5}}-\frac{50 \mu u_{1}^{5}}{\sqrt{n} Q^{* 5}}-\frac{91 \sqrt{n} \mu u_{1}^{5}}{Q^{* 7}} \\
& -\frac{45 n^{3 / 2} u_{1}^{5}}{16 Q^{* 3} \mu}+\frac{13 \sqrt{n} u_{1}^{5}}{8 Q^{* 3} \mu}+\frac{15 n^{3 / 2} u_{1}^{5}}{4 Q^{* 5} \mu}+\frac{15 n^{2} u_{1}^{5}}{32 Q^{* 2} \mu^{2}} \\
& -\frac{7 n u_{1}^{5}}{32 Q^{* 2} \mu^{2}}-\frac{3 n^{2} u_{1}^{5}}{8 Q^{* 4} \mu^{2}}-\frac{n^{3 / 2} u_{1}^{5}}{64 Q^{*} \mu^{3}}+\frac{661 n^{2} u_{1}^{5}}{96 Q^{* 4}} \\
& -\frac{251 n u_{1}^{5}}{16 Q^{* 4}}+\frac{3121 u_{1}^{5}}{480 Q^{* 4}}+\frac{n^{2} u_{1}^{5}}{256 \mu^{4}}-\frac{55 n^{2} u_{1}^{5}}{4 Q^{* 6}} \\
& +\frac{19 n u_{1}^{5}}{4 Q^{* 6}}+\frac{7 n^{2} u_{1}^{5}}{Q^{* 8}}-\frac{399 u_{2} \mu^{3} u_{1}^{4}}{n^{3 / 2} Q^{* 7}}+\frac{57 \mu^{3} u_{1}^{4}}{n^{3 / 2} Q^{* 6}} \\
& -\frac{180 u_{2} \mu^{2} u_{1}^{4}}{n Q^{* 6}}+\frac{30 \mu^{2} u_{1}^{4}}{n Q^{* 5}}-\frac{15 n u_{2} u_{1}^{4}}{Q^{* 4}}+\frac{9 u_{2} u_{1}^{4}}{Q^{* 4}} \\
& +\frac{30 n u_{2} u_{1}^{4}}{Q^{* 6}}-\frac{305 \sqrt{n} u_{2} \mu u_{1}^{4}}{4 Q^{* 5}}+\frac{30 u_{2} \mu u_{1}^{4}}{\sqrt{n} Q^{* 5}}+\frac{91 \sqrt{n} u_{2} \mu u_{1}^{4}}{Q^{* 7}} \quad \theta=-\frac{23134208 T^{5} \mu^{9}}{n^{9 / 2} Q^{* 9}}+\frac{57835520 T^{4} \mu^{9}}{n^{9 / 2} Q^{* 9}}-\frac{57835520 T^{3} \mu^{9}}{n^{9 / 2} Q^{* 9}} \\
& +\frac{61 \sqrt{n} \mu u_{1}^{4}}{4 Q^{* 4}}-\frac{6 \mu u_{1}^{4}}{\sqrt{n} Q^{* 4}}-\frac{13 \sqrt{n} \mu u_{1}^{4}}{Q^{* 6}}+\frac{45 n^{3 / 2} u_{2} u_{1}^{4}}{16 Q^{* 3} \mu} \\
& -\frac{9 \sqrt{n} u_{2} u_{1}^{4}}{8 Q^{* 3} \mu}-\frac{15 n^{3 / 2} u_{2} u_{1}^{4}}{4 Q^{* 5} \mu}-\frac{15 n^{3 / 2} u_{1}^{4}}{16 Q^{* 2} \mu}+\frac{3 \sqrt{n} u_{1}^{4}}{8 Q^{* 2} \mu} \\
& +\frac{3 n^{3 / 2} u_{1}^{4}}{4 Q^{* 4} \mu}-\frac{n u_{2} u_{1}^{4}}{8 Q^{* 2} \mu^{2}}+\frac{n u_{1}^{4}}{16 Q^{*} \mu^{2}}+\frac{15 n u_{1}^{4}}{4 Q^{* 3}} \\
& -\frac{9 u_{1}^{4}}{4 Q^{* 3}}-\frac{n^{3 / 2} u_{1}^{4}}{64 \mu^{3}}+\frac{n^{3 / 2} u_{2} u_{1}^{4}}{64 Q^{*} \mu^{3}}-\frac{5 n u_{1}^{4}}{Q^{* 5}} \\
& +\frac{15 n u_{2}^{2} u_{1}^{3}}{2 Q^{* 4}}-\frac{u_{2}^{2} u_{1}^{3}}{2 Q^{* 4}}-\frac{15 n u_{2}^{2} u_{1}^{3}}{Q^{* 6}}+\frac{90 u_{2}^{2} \mu^{2} u_{1}^{3}}{n Q^{* 6}} \\
& -\frac{30 u_{2} \mu^{2} u_{1}^{3}}{n Q^{* 5}}+\frac{6 \mu^{2} u_{1}^{3}}{n Q^{* 4}}-\frac{15 n u_{2} u_{1}^{3}}{4 Q^{* 3}}+\frac{u_{2} u_{1}^{3}}{4 Q^{* 3}} \\
& +\frac{5 n u_{2} u_{1}^{3}}{Q^{* 5}}+\frac{30 u_{2}^{2} \mu u_{1}^{3}}{\sqrt{n} Q^{* 5}}-\frac{12 u_{2} \mu u_{1}^{3}}{\sqrt{n} Q^{* 4}}+\frac{3 \mu u_{1}^{3}}{\sqrt{n} Q^{* 3}}
\end{aligned}
$$

$$
\begin{aligned}
& -\frac{3 \sqrt{n} u_{2}^{2} u_{1}^{3}}{4 Q^{* 3} \mu}+\frac{\sqrt{n} u_{2} u_{1}^{3}}{2 Q^{* 2} \mu}-\frac{\sqrt{n} u_{1}^{3}}{4 Q^{*} \mu}+\frac{5 n u_{1}^{3}}{4 Q^{* 2}} \\
& -\frac{u_{1}^{3}}{12 Q^{* 2}}+\frac{n u_{2}^{2} u_{1}^{3}}{16 Q^{* 2} \mu^{2}}+\frac{n u_{1}^{3}}{16 \mu^{2}}-\frac{n u_{2} u_{1}^{3}}{16 Q^{*} \mu^{2}} \\
& -\frac{n u_{1}^{3}}{Q^{* 4}}-\frac{4 u_{2}^{3} u_{1}^{2}}{Q^{* 4}}+\frac{3 u_{2}^{2} u_{1}^{2}}{Q^{* 3}}-\frac{2 u_{2} u_{1}^{2}}{Q^{* 2}} \\
& -\frac{10 u_{2}^{3} \mu u_{1}^{2}}{\sqrt{n} Q^{* 5}}+\frac{6 u_{2}^{2} \mu u_{1}^{2}}{\sqrt{n} Q^{* 4}}-\frac{3 u_{2} \mu u_{1}^{2}}{\sqrt{n} Q^{* 3}}+\frac{\mu u_{1}^{2}}{\sqrt{n} Q^{* 2}} \\
& +\frac{u_{1}^{2}}{Q^{*}}+\frac{\sqrt{n} u_{2}^{3} u_{1}^{2}}{4 Q^{* 3} \mu}-\frac{\sqrt{n} u_{2}^{2} u_{1}^{2}}{4 Q^{* 2} \mu}+\frac{\sqrt{n} u_{2} u_{1}^{2}}{4 Q^{*} \mu} \\
& -\frac{\sqrt{n} u_{1}^{2}}{4 \mu}+\frac{u_{2}^{4} u_{1}}{Q^{* 4}}-\frac{u_{2}^{3} u_{1}}{Q^{* 3}}+\frac{u_{2}^{2} u_{1}}{Q^{* 2}}-\frac{u_{2} u_{1}}{Q^{*}} .
\end{aligned}
$$

Finally, the center manifold can be expressed as

$$
+\frac{28917760 T^{2} \mu^{9}}{n^{9 / 2} Q^{* 9}}-\frac{7229440 T \mu^{9}}{n^{9 / 2} Q^{* 9}}+\frac{722944 \mu^{9}}{n^{9 / 2} Q^{* 9}}
$$$$
-\frac{7577600 T^{5} \mu^{7}}{n^{5 / 2} Q^{* 7}}+\frac{5627904 T^{5} \mu^{7}}{n^{7 / 2} Q^{* 7}}+\frac{6586368 T^{5} \mu^{7}}{n^{5 / 2} Q^{* 9}}
$$$$
+\frac{18944000 T^{4} \mu^{7}}{n^{5 / 2} Q^{* 7}}-\frac{12201984 T^{4} \mu^{7}}{n^{7 / 2} Q^{* 7}}-\frac{1634304 Q T^{4} \mu^{7}}{n^{7 / 2} Q^{* 8}}
$$$$
-\frac{16465920 T^{4} \mu^{7}}{n^{5 / 2} Q^{* 9}}-\frac{18944000 T^{3} \mu^{7}}{n^{5 / 2} Q^{* 7}}+\frac{10334208 T^{3} \mu^{7}}{n^{7 / 2} Q^{* 7}}
$$$$
+\frac{3268608 Q T^{3} \mu^{7}}{n^{7 / 2} Q^{* 8}}+\frac{16465920 T^{3} \mu^{7}}{n^{5 / 2} Q^{* 9}}+\frac{9472000 T^{2} \mu^{7}}{n^{5 / 2} Q^{* 7}}
$$$$
-\frac{4233216 T^{2} \mu^{7}}{n^{7 / 2} Q^{* 7}}-\frac{2451456 Q T^{2} \mu^{7}}{n^{7 / 2} Q^{* 8}}-\frac{8232960 T^{2} \mu^{7}}{n^{5 / 2} Q^{* 9}}
$$$$
-\frac{2368000 T \mu^{7}}{n^{5 / 2} Q^{* 7}}+\frac{824832 T \mu^{7}}{n^{7 / 2} Q^{* 7}}+\frac{817152 Q T \mu^{7}}{n^{7 / 2} Q^{* 8}}
$$ 


$$
\begin{aligned}
& +\frac{2058240 T \mu^{7}}{n^{5 / 2} Q^{* 9}}+\frac{236800 \mu^{7}}{n^{5 / 2} Q^{* 7}}-\frac{59136 \mu^{7}}{n^{7 / 2} Q^{* 7}} \\
& -\frac{102144 Q \mu^{7}}{n^{7 / 2} Q^{* 8}}-\frac{205824 \mu^{7}}{n^{5 / 2} Q^{* 9}}-\frac{676864 T^{5} \mu^{5}}{3 \sqrt{n} Q^{* 5}} \\
& +\frac{759808 T^{5} \mu^{5}}{n^{3 / 2} Q^{* 5}}-\frac{5899264 T^{5} \mu^{5}}{15 n^{5 / 2} Q^{* 5}}+\frac{450560 T^{5} \mu^{5}}{\sqrt{n} Q^{* 7}} \\
& -\frac{647168 T^{5} \mu^{5}}{n^{3 / 2} Q^{* 7}}-\frac{229376 T^{5} \mu^{5}}{\sqrt{n} Q^{* 9}}+\frac{1692160 T^{4} \mu^{5}}{3 \sqrt{n} Q^{* 5}} \\
& -\frac{1524736 T^{4} \mu^{5}}{n^{3 / 2} Q^{* 5}}+\frac{2064896 T^{4} \mu^{5}}{3 n^{5 / 2} Q^{* 5}}-\frac{312320 Q T^{4} \mu^{5}}{n^{3 / 2} Q^{* 6}} \\
& +\frac{245760 Q T^{4} \mu^{5}}{n^{5 / 2} Q^{* 6}}-\frac{1126400 T^{4} \mu^{5}}{\sqrt{n} Q^{* 7}}+\frac{1191936 T^{4} \mu^{5}}{n^{3 / 2} Q^{* 7}} \\
& +\frac{372736 Q T^{4} \mu^{5}}{n^{3 / 2} Q^{* 8}}+\frac{573440 T^{4} \mu^{5}}{\sqrt{n} Q^{* 9}}-\frac{1692160 T^{3} \mu^{5}}{3 \sqrt{n} Q^{* 5}} \\
& +\frac{1149952 T^{3} \mu^{5}}{n^{3 / 2} Q^{* 5}}-\frac{1373696 T^{3} \mu^{5}}{3 n^{5 / 2} Q^{* 5}}+\frac{624640 Q T^{3} \mu^{5}}{n^{3 / 2} Q^{* 6}} \\
& -\frac{384000 Q T^{3} \mu^{5}}{n^{5 / 2} Q^{* 6}}-\frac{46080 Q^{2} T^{3} \mu^{5}}{n^{5 / 2} Q^{* 7}}+\frac{1126400 T^{3} \mu^{5}}{\sqrt{n} Q^{* 7}} \\
& -\frac{765952 T^{3} \mu^{5}}{n^{3 / 2} Q^{* 7}}-\frac{745472 Q T^{3} \mu^{5}}{n^{3 / 2} Q^{* 8}}-\frac{573440 T^{3} \mu^{5}}{\sqrt{n} Q^{* 9}} \\
& +\frac{846080 T^{2} \mu^{5}}{3 \sqrt{n} Q^{* 5}}-\frac{387584 T^{2} \mu^{5}}{n^{3 / 2} Q^{* 5}}+\frac{438016 T^{2} \mu^{5}}{3 n^{5 / 2} Q^{* 5}} \\
& -\frac{468480 Q T^{2} \mu^{5}}{n^{3 / 2} Q^{* 6}}+\frac{207360 Q T^{2} \mu^{5}}{n^{5 / 2} Q^{* 6}}+\frac{69120 Q^{2} T^{2} \mu^{5}}{n^{5 / 2} Q^{* 7}} \\
& -\frac{563200 T^{2} \mu^{5}}{\sqrt{n} Q^{* 7}}+\frac{169984 T^{2} \mu^{5}}{n^{3 / 2} Q^{* 7}}+\frac{559104 Q T^{2} \mu^{5}}{n^{3 / 2} Q^{* 8}} \\
& +\frac{286720 T^{2} \mu^{5}}{\sqrt{n} Q^{* 9}}-\frac{211520 T \mu^{5}}{3 \sqrt{n} Q^{* 5}}+\frac{50048 T \mu^{5}}{n^{3 / 2} Q^{* 5}} \\
& -\frac{71488 T \mu^{5}}{3 n^{5 / 2} Q^{* 5}}+\frac{156160 Q T \mu^{5}}{n^{3 / 2} Q^{* 6}}-\frac{42240 Q T \mu^{5}}{n^{5 / 2} Q^{* 6}} \\
& -\frac{34560 Q^{2} T \mu^{5}}{n^{5 / 2} Q^{* 7}}+\frac{140800 T \mu^{5}}{\sqrt{n} Q^{* 7}}+\frac{10752 T \mu^{5}}{n^{3 / 2} Q^{* 7}} \\
& -\frac{186368 Q T \mu^{5}}{n^{3 / 2} Q^{* 8}}-\frac{71680 T \mu^{5}}{\sqrt{n} Q^{* 9}}+\frac{21152 \mu^{5}}{3 \sqrt{n} Q^{* 5}}-\frac{320 \mu^{5}}{n^{3 / 2} Q^{* 5}} \\
& +\frac{28832 \mu^{5}}{15 n^{5 / 2} Q^{* 5}}-\frac{19520 Q \mu^{5}}{n^{3 / 2} Q^{* 6}}+\frac{1920 Q \mu^{5}}{n^{5 / 2} Q^{* 6}}+\frac{5760 Q^{2} \mu^{5}}{n^{5 / 2} Q^{* 7}} \\
& -\frac{14080 \mu^{5}}{\sqrt{n} Q^{* 7}}-\frac{6400 \mu^{5}}{n^{3 / 2} Q^{* 7}}+\frac{23296 Q \mu^{5}}{n^{3 / 2} Q^{* 8}}+\frac{7168 \mu^{5}}{\sqrt{n} Q^{* 9}} \\
& -\frac{15360 T^{5} \mu^{3}}{\sqrt{n} Q^{* 3}}+\frac{9216 T^{5} \mu^{3}}{n^{3 / 2} Q^{* 3}}+\frac{12288 T^{5} \mu^{3}}{\sqrt{n} Q^{* 5}}
\end{aligned}
$$

$$
\begin{aligned}
& +\frac{23040 T^{4} \mu^{3}}{\sqrt{n} Q^{* 3}}-\frac{12800 T^{4} \mu^{3}}{n^{3 / 2} Q^{* 3}}+\frac{11520 Q T^{4} \mu^{3}}{\sqrt{n} Q^{* 4}} \\
& -\frac{7680 Q T^{4} \mu^{3}}{n^{3 / 2} Q^{* 4}}-\frac{12288 T^{4} \mu^{3}}{\sqrt{n} Q^{* 5}}-\frac{15360 Q T^{4} \mu^{3}}{\sqrt{n} Q^{* 6}} \\
& -\frac{14080 T^{3} \mu^{3}}{\sqrt{n} Q^{* 3}}+\frac{24320 T^{3} \mu^{3}}{3 n^{3 / 2} Q^{* 3}}-\frac{13440 Q T^{3} \mu^{3}}{\sqrt{n} Q^{* 4}} \\
& +\frac{7040 Q T^{3} \mu^{3}}{n^{3 / 2} Q^{* 4}}-\frac{3840 Q^{2} T^{3} \mu^{3}}{\sqrt{n} Q^{* 5}}+\frac{3328 Q^{2} T^{3} \mu^{3}}{n^{3 / 2} Q^{* 5}} \\
& +\frac{4608 T^{3} \mu^{3}}{\sqrt{n} Q^{* 5}}+\frac{12800 Q T^{3} \mu^{3}}{\sqrt{n} Q^{* 6}}+\frac{7680 Q^{2} T^{3} \mu^{3}}{\sqrt{n} Q^{* 7}} \\
& +\frac{5760 T^{2} \mu^{3}}{\sqrt{n} Q^{* 3}}-\frac{3200 T^{2} \mu^{3}}{n^{3 / 2} Q^{* 3}}+\frac{2880 Q T^{2} \mu^{3}}{\sqrt{n} Q^{* 4}} \\
& -\frac{1920 Q T^{2} \mu^{3}}{n^{3 / 2} Q^{* 4}}+\frac{5760 Q^{2} T^{2} \mu^{3}}{\sqrt{n} Q^{* 5}}-\frac{2688 Q^{2} T^{2} \mu^{3}}{n^{3 / 2} Q^{* 5}} \\
& -\frac{3840 T^{2} \mu^{3}}{\sqrt{n} Q^{* 5}}-\frac{640 Q^{3} T^{2} \mu^{3}}{n^{3 / 2} Q^{* 6}}+\frac{3840 Q T^{2} \mu^{3}}{\sqrt{n} Q^{* 6}} \\
& -\frac{11520 Q^{2} T^{2} \mu^{3}}{\sqrt{n} Q^{* 7}}-\frac{1920 T \mu^{3}}{\sqrt{n} Q^{* 3}}+\frac{640 T \mu^{3}}{n^{3 / 2} Q^{* 3}} \\
& +\frac{1440 Q T \mu^{3}}{\sqrt{n} Q^{* 4}}+\frac{480 Q T \mu^{3}}{n^{3 / 2} Q^{* 4}}-\frac{2880 Q^{2} T \mu^{3}}{\sqrt{n} Q^{* 5}} \\
& +\frac{192 Q^{2} T \mu^{3}}{n^{3 / 2} Q^{* 5}}+\frac{2688 T \mu^{3}}{\sqrt{n} Q^{* 5}}+\frac{640 Q^{3} T \mu^{3}}{n^{3 / 2} Q^{* 6}} \\
& -\frac{5760 Q T \mu^{3}}{\sqrt{n} Q^{* 6}}+\frac{5760 Q^{2} T \mu^{3}}{\sqrt{n} Q^{* 7}}+\frac{320 \mu^{3}}{\sqrt{n} Q^{* 3}}-\frac{64 \mu^{3}}{3 n^{3 / 2} Q^{* 3}} \\
& -\frac{480 Q \mu^{3}}{\sqrt{n} Q^{* 4}}-\frac{160 Q \mu^{3}}{n^{3 / 2} Q^{* 4}}+\frac{480 Q^{2} \mu^{3}}{\sqrt{n} Q^{* 5}}+\frac{160 Q^{2} \mu^{3}}{n^{3 / 2} Q^{* 5}} \\
& -\frac{576 \mu^{3}}{\sqrt{n} Q^{* 5}}-\frac{160 Q^{3} \mu^{3}}{n^{3 / 2} Q^{* 6}}+\frac{1280 Q \mu^{3}}{\sqrt{n} Q^{* 6}}-\frac{960 Q^{2} \mu^{3}}{\sqrt{n} Q^{* 7}} \\
& -\frac{128 T^{5} \mu}{\sqrt{n} Q^{*}}+\frac{192 T^{4} \mu}{\sqrt{n} Q^{*}}+\frac{64 Q T^{4} \mu}{\sqrt{n} Q^{* 2}}-\frac{160 T^{3} \mu}{\sqrt{n} Q^{*}} \\
& -\frac{32 Q T^{3} \mu}{\sqrt{n} Q^{* 2}}-\frac{32 Q^{2} T^{3} \mu}{\sqrt{n} Q^{* 3}}+\frac{48 T^{2} \mu}{\sqrt{n} Q^{*}}+\frac{48 Q T^{2} \mu}{\sqrt{n} Q^{* 2}} \\
& -\frac{16 Q^{2} T^{2} \mu}{\sqrt{n} Q^{* 3}}+\frac{16 Q^{3} T^{2} \mu}{\sqrt{n} Q^{* 4}}-\frac{24 T \mu}{\sqrt{n} Q^{*}}+\frac{24 Q T \mu}{\sqrt{n} Q^{* 2}} \\
& -\frac{40 Q^{2} T \mu}{\sqrt{n} Q^{* 3}}+\frac{24 Q^{3} T \mu}{\sqrt{n} Q^{* 4}}-\frac{8 Q^{4} T \mu}{\sqrt{n} Q^{* 5}}+\frac{12 \mu}{\sqrt{n} Q^{*}} \\
& -\frac{24 Q \mu}{\sqrt{n} Q^{* 2}}+\frac{28 Q^{2} \mu}{\sqrt{n} Q^{* 3}}-\frac{16 Q^{3} \mu}{\sqrt{n} Q^{* 4}}+\frac{4 Q^{4} \mu}{\sqrt{n} Q^{* 5}} \text {. }
\end{aligned}
$$


The dynamics on the center manifold is given by

$$
\begin{aligned}
& \frac{d u_{1}}{d N}=-\frac{39 \mu^{3} u_{1}^{4}\left(Q^{*}+5 u_{1}-5 u_{2}\right)}{n^{3 / 2} Q^{* 6}}-\frac{378 \mu^{4} u_{1}^{5}}{n^{2} Q^{* 7}}-\frac{3 \mu^{2} u_{1}^{3}\left(Q^{* 2}\left((124 n-53) u_{1}^{2}-96 u_{1} u_{2}+48 u_{2}^{2}\right)-112 n u_{1}^{2}+8 Q^{* 4}+24 Q^{* 3}\left(u_{1}-u_{2}\right)\right)}{4 n Q^{* 7}} \\
& +\frac{3 u_{1}^{3}\left(Q^{* 2}\left((6 n-3) u_{1}^{2}-2 u_{1} u_{2}+u_{2}^{2}\right)-6 n u_{1}^{2}+Q^{* 4}+Q^{* 3}\left(u_{1}-u_{2}\right)\right)}{4 Q^{* 5}} \\
& -\frac{3 \mu u_{1}^{2}\left(Q^{* 3}\left((2 n-1) u_{1}^{2}-2 u_{1} u_{2}+u_{2}^{2}\right)+Q^{* 2}\left(u_{1}-u_{2}\right)\left((6 n-5) u_{1}^{2}-2 u_{1} u_{2}+u_{2}^{2}\right)-2 n Q^{*} u_{1}^{2}+10 n u_{1}^{2}\left(u_{2}-u_{1}\right)+Q^{* 5}+Q^{* 4}\left(u_{1}-u_{2}\right)\right)}{\sqrt{n} Q^{* 6}}, \\
& \frac{d u_{2}}{d N}=-\frac{3 n^{5 / 2}\left(Q^{* 2}-1\right) u_{1}^{5}}{128 \mu^{3} Q^{* 2}}+\mu^{3}\left(\frac{3 u_{1}^{5}\left(63 n\left(Q^{* 2}-1\right)-65 Q^{* 2}\right)}{n^{3 / 2} Q^{* 8}}-\frac{39 u_{1}^{4}\left(Q^{*}-5 u_{2}\right)}{n^{3 / 2} Q^{* 6}}\right) \\
& +\frac{\frac{3 n^{3 / 2} u_{1}^{4}\left(Q^{*}-3 u_{2}\right)}{4 Q^{* 4}}+\frac{3 n^{3 / 2} u_{1}^{3}\left(-Q^{* 4}+Q^{* 2}-2 Q^{*} u_{2}+3 u_{2}^{2}\right)}{8 Q^{* 4}}-\frac{9 n^{3 / 2} u_{1}^{5}\left(4 n\left(Q^{* 2}-1\right)^{2}-3 Q^{* 4}+Q^{* 2}\right)}{16 Q^{* 6}}}{\mu} \\
& -\frac{378 \mu^{4} u_{1}^{5}}{n^{2} Q^{* 7}}+\frac{\frac{3 n^{2} u_{1}^{4}\left(Q^{* 3}-Q^{*}+2 u_{2}\right)}{32 Q^{* 3}}-\frac{3 n^{2} u_{1}^{5}}{16 Q^{* 3}}}{\mu^{2}}+\frac{u_{1}^{5}\left(72 n^{2}+3(2 n+1)(4 n-3) Q^{* 4}+6(1-16 n) n Q^{* 2}\right)}{4 Q^{* 7}} \\
& +\frac{3 u_{1}^{4}\left(4 n^{2}\left(Q^{* 2}-1\right)\left(Q^{*}\left(Q^{* 2}-2 Q^{*} u_{2}-1\right)+6 u_{2}\right)-2 n Q^{* 2}\left(2 Q^{* 3}-4 Q^{* 2} u_{2}+Q^{*}-4 u_{2}\right)+Q^{* 4}\left(Q^{*}-2 u_{2}\right)\right)}{4 Q^{* 7}} \\
& +\mu\left(\frac{u_{1}^{5}\left(336 n^{2}+3(n(124 n-125)+40) Q^{* 4}+3(101-236 n) n Q^{* 2}\right)}{8 \sqrt{n} Q^{* 8}}\right. \\
& +\frac{u_{1}^{4}\left(3 Q^{* 2}\left(Q^{*}-3 u_{2}\right)-6 n\left(Q^{*}-5 u_{2}\right)\right)}{\sqrt{n} Q^{* 6}}-\frac{3 u_{1}^{2}\left(Q^{*}-u_{2}\right)\left(Q^{* 2}+u_{2}^{2}\right)}{\sqrt{n} Q^{* 4}} \\
& \left.+\frac{3 u_{1}^{3}\left(n\left(Q^{* 4}-2 Q^{* 3} u_{2}+Q^{* 2}\left(3 u_{2}^{2}-1\right)+4 Q^{*} u_{2}-10 u_{2}^{2}\right)-Q^{* 2}\left(Q^{* 2}-2 Q^{*} u_{2}+3 u_{2}^{2}\right)\right)}{\sqrt{n} Q^{* 6}}\right) \\
& +\frac{3 u_{1}^{3}\left(Q^{* 2}\left(Q^{* 2}-Q^{*} u_{2}+u_{2}^{2}\right)-4 n\left(Q^{* 2}-3 Q^{*} u_{2}+6 u_{2}^{2}\right)\right)}{4 Q^{* 5}} \\
& +\mu^{2}\left(\frac{3 u_{1}^{5}\left(\left(\frac{53}{n}-20\right) Q^{* 2}-44\right)}{4 Q^{* 7}}+\frac{3 u_{1}^{4}\left(13 n\left(Q^{*}\left(Q^{* 2}-4 Q^{*} u_{2}-1\right)+6 u_{2}\right)-12 Q^{* 2}\left(Q^{*}-4 u_{2}\right)\right)}{2 n Q^{* 7}}\right. \\
& \left.-\frac{6 u_{1}^{3}\left(Q^{* 2}-3 Q^{*} u_{2}+6 u_{2}^{2}\right)}{n Q^{* 5}}\right)+\frac{3 n u_{1}^{2}\left(Q^{* 5}-Q^{* 3}+2 Q^{* 2} u_{2}-3 Q^{*} u_{2}^{2}+4 u_{2}^{3}\right)}{2 Q^{* 5}} .
\end{aligned}
$$

In the Fig. 13 are presented some numerical solutions of the system (285), (286) for $n=2,4, \mu=\frac{1}{2}, 1$ and $Q^{*}=$ \pm 1 . The plot illustrates the generic feature that for $Q^{*}>$ 0 (respectively, $Q^{*}<0$ ), the center manifold is unstable (respectively, stable), but in this case the third eigenvalue is $-\frac{3}{2} Q^{*}<0$ (respectively, $-\frac{3}{2} Q^{*}>0$ ). This means the the origin is a saddle. Using the Theorem 2, we conclude that the center manifold of origin for the system (242), (243), and the origin are unstable (saddle).

\section{Case 4: $k \neq 0, \Lambda \neq 0$ under the detailed-balance condition}

In this case the autonomous system writes:

$$
\begin{aligned}
\frac{d x}{d N}= & \sqrt{6} s\left[-x^{2}+(u-z)^{2}+1\right] \\
& +x\left[3 x^{2}+2(u-z) z-3\right], \\
\frac{d z}{d N}= & z\left[3 x^{2}+2(u-z) z-2\right], \\
\frac{d u}{d N}=u\left[3 x^{2}+2(u-z) z\right], & \\
\frac{d s}{d N}= & -2 \sqrt{6} x f(s) .
\end{aligned}
$$



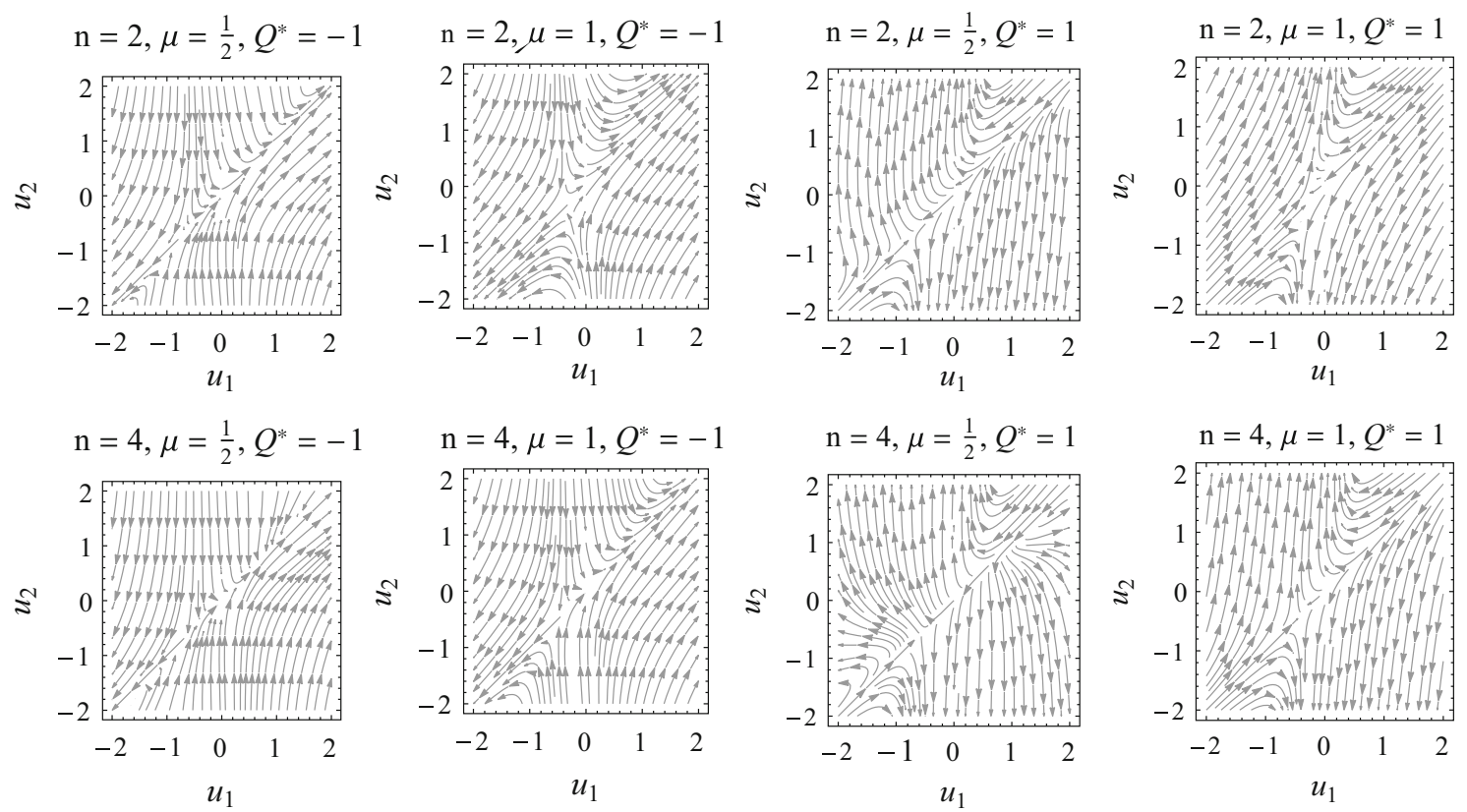

Fig. 13 Numerical solutions of the system (285), (286)

Table 7 Case 4: Equilibrium points at the finite region of the system (287), (288), (289), (290)

\begin{tabular}{|c|c|c|c|c|}
\hline Equil. points & $(x, z, u, s)$ & Existence & Eigenvalues & Stability \\
\hline$P_{14}(\hat{s})$ & $(1,0,0, \hat{s})$ & $f(\hat{s})=0$ & $6-2 \sqrt{6} \hat{s}, 1,3,-2 \sqrt{6} f^{\prime}(\hat{s})$ & $\begin{array}{l}\text { Nonhyperbolic for } f^{\prime}(\hat{s})=0 \text {, or } \hat{s}=\sqrt{\frac{3}{2}} \\
\text { Source for } f^{\prime}(\hat{s})<0, \hat{s}<\sqrt{\frac{3}{2}} \\
\text { Saddle otherwise }\end{array}$ \\
\hline$P_{15}(\hat{s})$ & $(-1,0, \hat{s})$ & $f(\hat{s})=0$ & $6+2 \sqrt{6} \hat{s}, 1,3,2 \sqrt{6} f^{\prime}(\hat{s})$ & $\begin{array}{l}\text { Nonhyperbolic for } f^{\prime}(\hat{s})=0 \text {, or } \hat{s}=-\sqrt{\frac{3}{2}} \\
\text { Source for } f^{\prime}(\hat{s})>0, \hat{s}>-\sqrt{\frac{3}{2}} \\
\text { Saddle otherwise }\end{array}$ \\
\hline$P_{16}(\hat{s})$ & $\left(\sqrt{\frac{2}{3}} \hat{s}, 0,0, \hat{s}\right)$ & $-\sqrt{\frac{3}{2}} \leq \hat{s} \leq \sqrt{\frac{3}{2}}$ & $2 \hat{s}^{2}-3,2\left(\hat{s}^{2}-1\right), 2 \hat{s}^{2},-4 \hat{s} f^{\prime}(\hat{s})$ & $\begin{array}{l}\text { Nonhyperbolic for } f^{\prime}(\hat{s})=0 \text {, or } \\
\qquad \hat{s} \in\left\{-\sqrt{\frac{3}{2}},-1,0,1, \sqrt{\frac{3}{2}}\right\} \\
\text { Saddle otherwise }\end{array}$ \\
\hline$P_{16}^{0}\left(u_{c}\right)$ & $\left(0,0, u_{c}, 0\right)$ & Always & $-2,0,-\frac{3}{2} \pm \frac{1}{2} \sqrt{9-48 f(0)\left(u_{c}^{2}+1\right)}$ & Nonhyperbolic \\
\hline$P_{17,18}(\hat{s})$ & $\left(\frac{\sqrt{\frac{2}{3}}}{\hat{s}}, \pm \frac{\sqrt{1-\hat{s}^{2}}}{\hat{s}}, 0, \hat{s}\right)$ & $-1 \leq \hat{s} \leq 1, \hat{s} \neq 0$ & $2,-\frac{1}{2} \pm \frac{\sqrt{16 \hat{s}^{2}-15 \hat{s}^{4}}}{2 \hat{s}^{2}},-\frac{4 f^{\prime}(\hat{s})}{\hat{s}}$ & $\begin{array}{l}\text { Nonhyperbolic for } f^{\prime}(\hat{s})=0 \text {, or } \hat{s} \in\{-1,1\} \\
\text { Saddle otherwise }\end{array}$ \\
\hline
\end{tabular}

where the prime means derivative with respect to $\ln a$, defined on the phase space $\left\{(x, z, u, s) \in \mathbb{R}^{3}: x^{2}-(u-k z)^{2} \leq 1\right\}$. The equilibrium points/curves at the finite region of the phase space are presented in Table 7, where is shown the existence and stability conditions. We proceed to the discussion of the more relevant features of them.

- $P_{14}(\hat{s}):(x, z, u, s)=(1,0,0, \hat{s})$, where $f(\hat{s})=0$. It is a source for $f^{\prime}(\hat{s})<0, \hat{s}<\sqrt{\frac{3}{2}}$.
- $P_{15}(\hat{s}):(x, z, s)=(-1,0, \hat{s})$, where $f(\hat{s})=0$. It is a source for $f^{\prime}(\hat{s})>0, \hat{s}>-\sqrt{\frac{3}{2}}$.

- $P_{16}(\hat{s}):(x, z, u, s)=\left(\sqrt{\frac{2}{3}} \hat{s}, 0,0, \hat{s}\right)$, where $f(\hat{s})=0$, $-\sqrt{\frac{3}{2}} \leq \hat{s} \leq \sqrt{\frac{3}{2}}$. This point is reduced to $P_{16}$ studied in [41]. It is generically a saddle.

- $P_{16}^{0}\left(u_{c}\right):(x, z, u, s)=\left(0,0, u_{c}, 0\right)$. This point is new, and it was not found in [41]. It is nonhyperbolic. 
- $P_{17,18}(\hat{s}):\left(\frac{\sqrt{\frac{2}{3}}}{\hat{s}}, \pm \frac{\sqrt{1-\hat{s}^{2}}}{\hat{s}}, 0, \hat{s}\right)$, where $f(\hat{s})=0$, $-1 \leq \hat{s} \leq 1, \hat{s} \neq 0$. It is generically a saddle.

- There are four lines of equilibrium points $P_{19,20}\left(s_{c}\right)$ : $(x, z, u, s)=\left(0,0, \pm i, s_{c}\right), s_{c} \in \mathbb{R}$ and $P_{21,22}\left(s_{c}\right):$ $(x, z, u, s)=\left(0, \pm i, 0, s_{c}\right), s_{c} \in \mathbb{R}$ which are not considered since they are complex valued.

\section{Beyond the detailed-balance condition}

Using the variables (12) the corresponding autonomous system is found to be:

$$
\begin{aligned}
& \frac{d x_{2}}{d N}=2 x_{2}\left(3 x^{2}+x_{2}+2 x_{3}+3 x_{4}-1\right), \\
& \frac{d x_{3}}{d N}=2 x_{3}\left(3 x^{2}+x_{2}+2 x_{3}+3 x_{4}-2\right), \\
& \frac{d x_{4}}{d N}=2 x_{4}\left(3 x^{2}+x_{2}+2 x_{3}+3 x_{4}-3\right), \\
& \frac{d x}{d N}=3 x^{3}+\left(x_{2}+2 x_{3}+3 x_{4}-3\right) x+\sqrt{6} s y^{2}, \\
& \frac{d y}{d N}=\left(3 x^{2}-\sqrt{6} s x+x_{2}+2 x_{3}+3 x_{4}\right) y, \\
& \frac{d s}{d N}=-2 \sqrt{6} x f(s),
\end{aligned}
$$

defining a dynamical system in $\mathbb{R}^{6}$.

\subsection{Arbitrary potentials}

The stability of the hyperbolic equilibrium points is given by analyzing the signs of the reals parts of the eigenvalues of the matrix of linear perturbations $\mathbf{Q}$ evaluated at each equilibrium point. The results are shown in Table 8 . The equilibrium points/curves at the finite region of the phase space are the following:

- $P_{23}(\hat{s}):\left(x_{2}, x_{3}, x_{4}, x, y, s\right)=\left(0,0,1-x_{c}^{2}, x_{c}, 0, \hat{s}\right)$. Exists for $\hat{s}$ such that $f(\hat{s})=0$. The eigenvalues are $6,0,2,4,3-\sqrt{6} \hat{s} x_{c},-2 \sqrt{6} x_{c} f^{\prime}(\hat{s})$.

- $P_{24,25}(\hat{s}):\left(x_{2}, x_{3}, x_{4}, x, y, s\right)=(0,0,0, \pm 1,0, \hat{s})$. Exists for $\hat{s}$ such that $f(\hat{s})=0$. The eigenvalues are $6,0,2,4,3 \mp \sqrt{6} \hat{s}, \mp 2 \sqrt{6} f^{\prime}(\hat{s})$.

- $P_{26}\left(y_{c}\right):\left(x_{2}, x_{3}, x_{4}, x, y, s\right)=\left(0,0,0,0, y_{c}, 0\right)$. Always exists. The eigenvalues are $-6,-4,-2,0$, $-\frac{1}{2}\left(3+\sqrt{9-48 f(0) y_{c}^{2}}\right),-\frac{1}{2}\left(3-\sqrt{9-48 f(0) y_{c}^{2}}\right)$.

- $P_{26}^{0}:\left(x_{2}, x_{3}, x_{4}, x, y, s\right)=(0,0,0,0,0,0)$. Always exists. The eigenvalues are $-6,-4,-3,-2,0,0$.

$-P_{27,28}(\hat{s}): \quad\left(x_{2}, x_{3}, x_{4}, x, y, s\right)=\left(0,0,0, \sqrt{\frac{2}{3}} \hat{s}\right.$, $\left.\pm \sqrt{1-\frac{2 \hat{s}^{2}}{3}}, \hat{s}\right)$. Exists for $\hat{s}$ such that $f(\hat{s})=0$. The eigenvalues are $4 \hat{s}^{2}, 2 \hat{s}^{2}-3,2\left(2 \hat{s}^{2}-3\right)$, $-4\left(1-\hat{s}^{2}\right), 2\left(2 \hat{s}^{2}-1\right),-4 \hat{s} f^{\prime}(\hat{s})$.

- $P_{29}\left(s_{c}\right):\left(x_{2}, x_{3}, x_{4}, x, y, s\right)=\left(1,0,0,0,0, s_{c}\right)$. It always exists. The eigenvalues are $-4,-2,-2,2,1,0$.

$-P_{30,31}(\hat{s}):\left(x_{2}, x_{3}, x_{4}, x, y, s\right)=\left(1-\frac{1}{2 \hat{s}^{2}}, 0,0\right.$, $\left.\frac{1}{\sqrt{6} \hat{s}}, \pm \frac{1}{\sqrt{3} \hat{s}}, \hat{s}\right)$. Exists for $\hat{s} \neq 0$ such that $f(\hat{s})=0$. The eigenvalues are $-4,-2,2,-\frac{\hat{s}+\sqrt{2-3 \hat{s}^{2}}}{\hat{s}}, \frac{\sqrt{2-3 \hat{s}^{2}}-\hat{s}}{\hat{s}}$, $-\frac{2 f^{\prime}(\hat{s})}{\hat{s}}$.

- $P_{32}\left(s_{c}\right):\left(x_{2}, x_{3}, x_{4}, x, y, s\right)=\left(0,1,0,0,0, s_{c}\right)$. Exists for all $s_{c}$. The eigenvalues are $4,-2,2,2,-1,0$.

- $P_{33,34}(\hat{s}):\left(x_{2}, x_{3}, x_{4}, x, y, s\right)=\left(0,1-\frac{1}{\hat{s}^{2}}, 0, \frac{\sqrt{\frac{2}{3}}}{\hat{s}}\right.$, $\left.\pm \frac{1}{\sqrt{3} \hat{s}}, \hat{s}\right)$. Exists for $\hat{s} \neq 0$ such that $f(\hat{s})=0$. The eigenvalues are $4,-2,2,-\frac{\hat{s}+\sqrt{16-15 \hat{s}^{2}}}{2 \hat{s}}, \frac{\sqrt{16-15 \hat{s}^{2}}-\hat{s}}{2 \hat{s}}$, $-\frac{4 f^{\prime}(\hat{s})}{\hat{s}}$.

$-P_{35}(\hat{s}):\left(x_{2}, x_{3}, x_{4}, x, y, s\right)=\left(0,0,1-\frac{3}{2 \hat{s}^{2}}, \frac{\sqrt{\frac{3}{2}}}{\hat{s}}, 0, \hat{s}\right)$. Exists for $\hat{s}$ such that $f(\hat{s})=0$. The eigenvalues are $0,0,2,4,6,-\frac{6 f^{\prime}(\hat{s})}{\hat{s}}$.

Owing to the fact that the dynamical system (291), (292), (293), (294), (295), (296), is unbounded, we use the compact variables

$X_{2}=\frac{x_{2}}{\sqrt{1+r^{2}}}, \quad X_{3}=\frac{x_{3}}{\sqrt{1+r^{2}}}, \quad X_{4}=\frac{x_{4}}{\sqrt{1+r^{2}}}$,

$X=\frac{x}{\sqrt{1+r^{2}}}, \quad Y=\frac{y}{\sqrt{1+r^{2}}}, \quad S=\frac{2}{\pi} \arctan (s)$,

$r=\sqrt{x_{2}^{2}+x_{3}^{2}+x_{4}^{2}+x^{2}+y^{2}}$,

and the time rescaling

$f^{\prime}=\left(1-X_{2}^{2}-X_{3}^{2}-X_{4}^{2}-X^{2}-Y^{2}\right) d f / d N$,

such that we obtain the system

$$
\begin{aligned}
& \frac{d X_{2}}{d \tau}=3 X^{2} X_{2}\left(X^{2}+Y^{2}\right) \\
& \quad+P X_{2}\left(X^{2}+Y^{2}\right)\left(X_{2}+2 X_{3}+3 X_{4}\right) \\
& \quad+P^{2} X_{2}\left(7 X^{2}+2 X_{3}^{2}+4 X_{4}^{2}-2 Y^{2}\right) \\
& \quad+2 P^{3} X_{2}\left(X_{2}+2 X_{3}+3 X_{4}-P\right) \\
& \frac{d X_{3}}{d \tau}=3 X^{2} X_{3}\left(X^{2}+Y^{2}\right) \\
& \quad+P X_{3}\left(X^{2}+Y^{2}\right)\left(X_{2}+2 X_{3}+3 X_{4}\right) \\
& \quad+P^{2} X_{3}\left(5 X^{2}-2 X_{2}^{2}+2 X_{4}^{2}-4 Y^{2}\right) \\
& \quad+2 P^{3} X_{3}\left(X_{2}+2 X_{3}+3 X_{4}-2 P\right)
\end{aligned}
$$




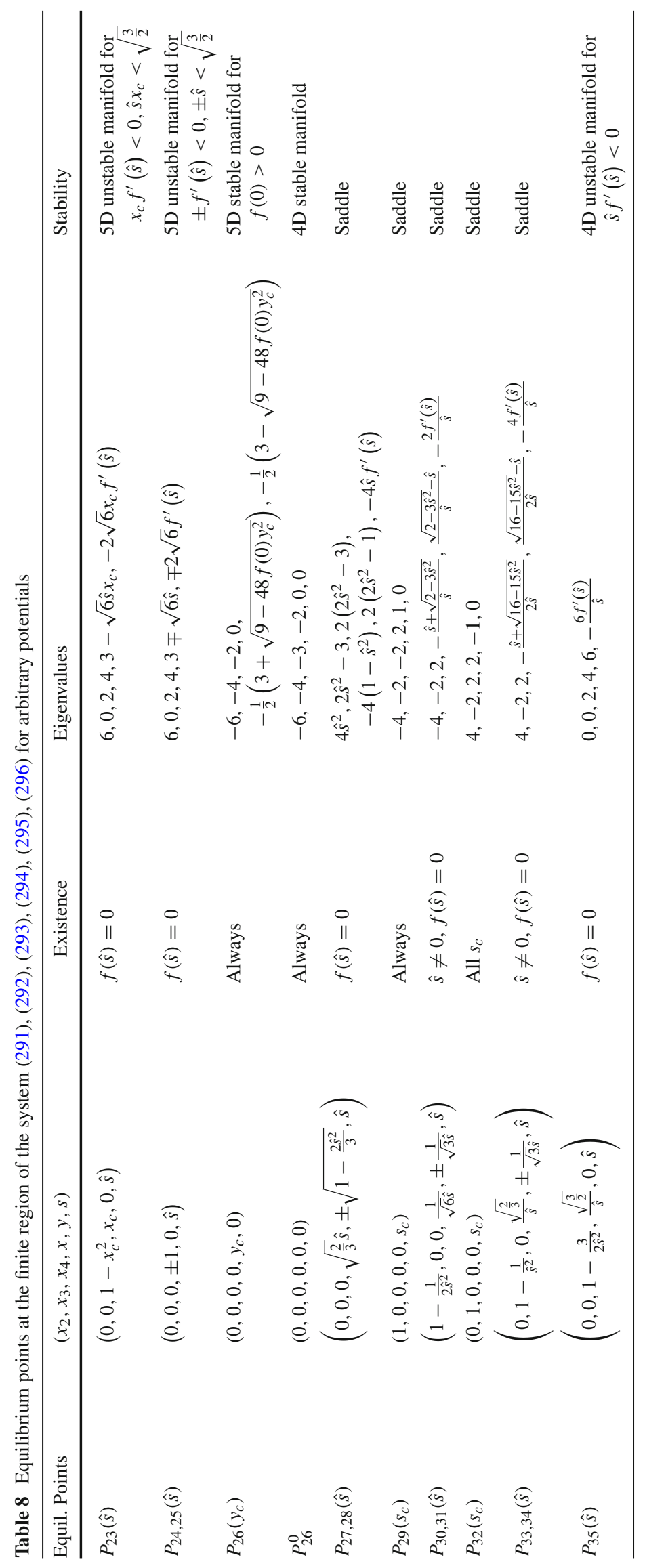




$$
\begin{aligned}
& \frac{d X_{4}}{d \tau}=3 X^{2} X_{4}\left(X^{2}+Y^{2}\right) \\
& \quad+P X_{4}\left(X^{2}+Y^{2}\right)\left(X_{2}+2 X_{3}+3 X_{4}\right) \\
& \quad+P^{2} X_{4}\left(3 X^{2}-2\left(2 X_{2}^{2}+X_{3}^{2}+3 Y^{2}\right)\right) \\
& \quad+2 P^{3} X_{4}\left(X_{2}+2 X_{3}+3 X_{4}-3 P\right) \\
& \frac{d X}{d \tau}=-3 X^{3}\left(X_{2}^{2}+X_{3}^{2}+X_{4}^{2}\right) \\
& \quad+P\left[-X\left(X_{2}+2 X_{3}+3 X_{4}\right)\left(X_{2}^{2}+X_{3}^{2}+X_{4}^{2}\right)\right. \\
& \left.\quad+\sqrt{6} Y^{2} \tan \left(\frac{\pi S}{2}\right)\right] \\
& \quad+P^{2} X\left(3 X^{2}-X_{2}^{2}+X_{3}^{2}+3 X_{4}^{2}-3 Y^{2}\right) \\
& \quad+P^{3} X\left(X_{2}+2 X_{3}+3 X_{4}-3 P\right), \\
& \frac{d Y}{d \tau}=-3 X^{2} Y\left(X_{2}^{2}+X_{3}^{2}+X_{4}^{2}\right) \\
& \quad+P Y\left[\left(-X_{2}-2 X_{3}-3 X_{4}\right)\left(X_{2}^{2}+X_{3}^{2}+X_{4}^{2}\right)\right. \\
& \left.\quad-\sqrt{6} X \tan \left(\frac{\pi S}{2}\right)\right]+2 P^{2} Y\left(3 X^{2}+X_{2}^{2}+2 X_{3}^{2}+3 X_{4}^{2}\right) \\
& \quad+P^{3} Y\left(X_{2}+2 X_{3}+3 X_{4}\right), \\
& \frac{d S}{d \tau}=-\frac{2 \sqrt{6} P X(\cos (\pi S)+1) f\left(\tan \left(\frac{\pi S}{2}\right)\right)}{\pi}
\end{aligned}
$$

where $P=\sqrt{1-X_{2}^{2}-X_{3}^{2}-X_{4}^{2}-X^{2}-Y^{2}}$.

The invariant sets at infinity are the cylinders $X=0, X_{2}^{2}+$ $X_{3}^{2}+X_{4}^{2}+Y^{2}=1, S \in[-1,1]$ and $X_{2}=X_{3}=X_{4}=$ $0, X^{2}+Y^{2}=1, S \in[-1,1]$.

After taking a time rescaling, the dynamics on the invariant set $X_{2}=X_{3}=X_{4}=0$ is given by

$$
\begin{aligned}
& \frac{d X}{d \tau}=\sqrt{6} Y^{2} \tan \left(\frac{\pi S}{2}\right)+3 X\left(2 X^{2}-1\right) \sqrt{1-X^{2}-Y^{2}} \\
& \frac{d Y}{d \tau}=-X Y\left(\sqrt{6} \tan \left(\frac{\pi S}{2}\right)-6 X \sqrt{1-X^{2}-Y^{2}}\right) \\
& \frac{d S}{d \tau}=-\frac{2 \sqrt{6} X(\cos (\pi S)+1) f\left(\tan \left(\frac{\pi S}{2}\right)\right)}{\pi}
\end{aligned}
$$

This invariant set is relevant concerning the future asymptotic dynamics. It contains the de Sitter solutions $P_{26}\left(y_{c}\right)$, and some relevant invariant sets at the infinity region.

\subsection{Exponential potential}

In this case we obtain the autonomous system (291), (292), (293), (294), (295), (296) transforms to:

$$
\frac{d x_{2}}{d N}=2 x_{2}\left(3 x^{2}+x_{2}+2 x_{3}+3 x_{4}-1\right)
$$

$$
\begin{aligned}
& \frac{d x_{3}}{d N}=2 x_{3}\left(3 x^{2}+x_{2}+2 x_{3}+3 x_{4}-2\right), \\
& \frac{d x_{4}}{d N}=2 x_{4}\left(3 x^{2}+x_{2}+2 x_{3}+3 x_{4}-3\right), \\
& \frac{d x}{d N}=3 x^{3}+\left(x_{2}+2 x_{3}+3 x_{4}-3\right) x+\sqrt{6} s y^{2}, \\
& \frac{d y}{d N}=\left(3 x^{2}-\sqrt{6} s x+x_{2}+2 x_{3}+3 x_{4}\right) y, \\
& 1=x_{1}+x_{2}+x_{3}+x_{4}+x^{2}+y^{2}
\end{aligned}
$$

The equilibrium points of the system (308), (309), (310), (311), (312) and their behavior [41] is summarized as follows. The point $P_{35}$ studied in [41] is omitted. It is a special case of the line $P_{23}$. The stability of the hyperbolic equilibrium points of this system have been extensively studied in [41]. Now we summarize the previous findings. The unstable equilibrium points $P_{27,28}, P_{29}$ correspond to dark matter domination, and the unstable point $P_{32}$ corresponds to an unphysical dark-energy dominated universe, and the unstable $P_{30,31}, P_{33,34}$ which have physical $w_{M}, w_{D E}$ but dependent on the specific dark-matter form. The system admits also the line of equilibrium points $P_{23}$, which is nonhyperbolic with positive non-null eigenvalues, thus unstable, with furthermore unphysical cosmological quantities. Additionally, points $P_{24,25}$ are also dark-matter dominated, unstable nonhyperbolic ones. Due to $\sigma_{3}$ has an arbitrary sign, $P_{33,34}$ could also correspond to an oscillatory universe, for a wide region of the parameters $\sigma_{3}$ and $s$. However, this oscillatory behavior has a small probability to be the late-time state of the universe because it is not stable (with at least two positive eigenvalues). The scenario at hand admits a final equilibrium point, namely $P_{26}$, representing a de Sitter solution. As we show in detail in the next Sect. 7.2.1 by using Center Manifold Theory, it is indeed a locally asymptotically stable and thus it can be a late-time attractor of HL universe beyond the detailed-balance.

\subsubsection{Stability analysis of the de Sitter solution in Hoŕava-Lifshitz cosmology without detailed-balance for the exponential potential}

In order to analyze the stability of de Sitter solution we can use center manifold theorem.

Proposition 11 The origin for the system (312) is locally asymptotically stable.

Proof Defining $\left(u, v_{1}, v_{2}, v_{3}, v_{4}\right):=\left(y, x_{4}, x_{3}, x, x_{2}\right)$, the linear part of the vector field is transformed into its Jordan canonical form: 


$$
\left(\begin{array}{c}
\frac{d u}{d \tau} \\
\frac{d v_{1}}{d \tau} \\
\frac{d v_{2}}{d \tau} \\
\frac{d v_{3}}{d \tau} \\
\frac{d v_{4}}{d \tau}
\end{array}\right)=\left(\begin{array}{ccccc}
0 & 0 & 0 & 0 & 0 \\
0 & -6 & 0 & 0 & 0 \\
0 & 0 & -4 & 0 & 0 \\
0 & 0 & 0 & -3 & 0 \\
0 & 0 & 0 & 0 & -2
\end{array}\right)\left(\begin{array}{c}
u \\
v_{1} \\
v_{2} \\
v_{3} \\
v_{4}
\end{array}\right)+\left(\begin{array}{c}
f(u, \mathbf{v}) \\
g_{1}(u, \mathbf{v}) \\
g_{2}(u, \mathbf{v}) \\
g_{3}(u, \mathbf{v}) \\
g_{4}(u, \mathbf{v})
\end{array}\right)
$$

where $f(u, \mathbf{v})=u\left(3 v_{1}+2 v_{2}-\sqrt{6} s v_{3}+4 v_{4}\right)$,

$$
\begin{aligned}
& g_{1}(u, \mathbf{v})=2 v_{1}\left(3 v_{3}^{2}+3 v_{1}+2 v_{2}+v_{4}\right), \\
& g_{2}(u, \mathbf{v})=2 v_{2}\left(3 v_{3}^{2}+3 v_{1}+2 v_{2}+v_{4}\right), \\
& g_{3}(u, \mathbf{v})=\sqrt{6} s u^{2}+v_{3}\left(3 v_{3}^{2}+3 v_{1}+2 v_{2}+v_{4}\right),
\end{aligned}
$$

and $g_{4}(u, \mathbf{v})=2 v_{4}\left(3 v_{3}^{2}+3 v_{1}+2 v_{2}+v_{4}\right)$.

The system (314) is written in diagonal form

$$
\begin{aligned}
& \frac{d u}{d \tau}=C u+f(u, \mathbf{v}) \\
& \frac{d \mathbf{v}}{d \tau}=P \mathbf{v}+\mathbf{g}(u, \mathbf{v}),
\end{aligned}
$$

where $(u, \mathbf{v}) \in \mathbb{R} \times \mathbb{R}^{4}, C$ is the zero $1 \times 1$ matrix, $P$ is a $4 \times 4$ matrix with negative eigenvalues and $f, \mathbf{g}$ vanish at $\mathbf{0}$ and have vanishing derivatives at $\mathbf{0}$. According to Theorem 1, there exists a 1-dimensional invariant local center manifold $W^{c}(\mathbf{0})$ of (315) tangent to the center subspace (the $\mathbf{v}=\mathbf{0}$ space) at $\mathbf{0}$. Moreover, $W^{c}(\mathbf{0})=$ $\left\{(u, \mathbf{v}) \in \mathbb{R} \times \mathbb{R}^{4}: \mathbf{v}=\mathbf{h}(u), \mathbf{h}(0)=\mathbf{0}, D \mathbf{h}(0)=\mathbf{0}\right\},|u|$ $<\delta$ for $\delta$ sufficiently small. The restriction of (315) to the center manifold is $\frac{d u}{d \tau}=f(u, \mathbf{h}(u))$, where the function $\mathbf{h}(u)$ that defines the local center manifold satisfies

$$
D \mathbf{h}(u)[f(u, \mathbf{h}(u))]-P \mathbf{h}(u)-\mathbf{g}(u, \mathbf{h}(u))=0 .
$$

The vectorial Eq. (316) can be written as the system of ODE

$$
\begin{aligned}
& 2 h_{1}\left(3 h_{1}+2 h_{2}+3 h_{3}^{2}+h_{4}-3\right) \\
& -u h_{1}^{\prime}(u)\left(-\sqrt{6} s h_{3}+3 h_{1}+2 h_{2}+3 h_{3}^{2}+h_{4}\right)=0, \\
& 2 h_{2}\left(3 h_{1}+2 h_{2}+3 h_{3}^{2}+h_{4}-2\right) \\
& -u h_{2}^{\prime}(u)\left(-\sqrt{6} s h_{3}+3 h_{1}+2 h_{2}+3 h_{3}^{2}+h_{4}\right)=0, \\
& \sqrt{6} s u^{2}+h_{3}\left(3 h_{1}+2 h_{2}+3 h_{3}^{2}+h_{4}-3\right) \\
& -u h_{3}^{\prime}(u)\left(-\sqrt{6} s h_{3}+3 h_{1}+2 h_{2}+3 h_{3}^{2}+h_{4}\right)=0,
\end{aligned}
$$

$$
\begin{aligned}
& 2 h_{4}\left(3 h_{1}+2 h_{2}+3 h_{3}^{2}+h_{4}-1\right) \\
& -u h_{4}^{\prime}(u)\left(-\sqrt{6} s h_{3}+3 h_{1}+2 h_{2}+3 h_{3}^{2}+h_{4}\right)=0 .
\end{aligned}
$$

The equation (7.2.1) can be solved approximately by expanding $\mathbf{h}(u)$ in Taylor series at $u=0$. Due to $\mathbf{h}(0)=\mathbf{0}$ and $D \mathbf{h}(0)=\mathbf{0}$, we substitute

$\mathbf{h}(u):=\left(\begin{array}{c}h_{1}(u) \\ h_{2}(u) \\ h_{3}(u) \\ h_{4}(u)\end{array}\right)=\left(\begin{array}{c}\sum_{j=1}^{10} a_{j} u^{j+1}+O\left(u^{12}\right) \\ \sum_{j=1}^{10} b_{j} u^{j+1}+O\left(u^{12}\right) \\ \sum_{j=1}^{10} c_{j} u^{j+1}+O\left(u^{12}\right) \\ \sum_{j=1}^{10} d_{j} u^{j+1}+O\left(u^{12}\right)\end{array}\right)$,

into (316) and set the coefficients of like powers of $u$ equal to zero to find the unknowns $a_{1}, b_{1}, c_{1}, d_{1}, \ldots$ The non-zero coefficients are

$$
\begin{aligned}
& c_{1}=\sqrt{\frac{2}{3}} s, c_{3}=\frac{4}{3} \sqrt{\frac{2}{3}} s^{3}, c_{5}=\frac{2}{3} \sqrt{\frac{2}{3}} s^{3}\left(8 s^{2}-1\right), \\
& c_{7}=\frac{8}{27} \sqrt{\frac{2}{3}} s^{5}\left(112 s^{2}-27\right), \\
& c_{9}=\frac{4}{81} \sqrt{\frac{2}{3}} s^{5}\left(5440 s^{4}-1872 s^{2}+63\right) .
\end{aligned}
$$

Therefore, the dynamics on the center manifold is governed by the gradient-like equation

$$
\begin{aligned}
& \frac{d U}{d \tau}=-\nabla U(u), \quad U(u)=\frac{s^{2} u^{4}}{2}+\frac{1}{9} s^{2}\left(4 s^{2}-3\right) u^{6} \\
& +\frac{4}{135} s^{4}\left(224 s^{4}-138 s^{2}+9\right) u^{10}+\frac{1}{6} s^{4}\left(8 s^{2}-5\right) u^{8} \\
& +\frac{2}{243} s^{6}\left(5440 s^{4}-3504 s^{2}+423\right) u^{12}
\end{aligned}
$$

for which the origin is a degenerate minimum. Using the Theorem 2, we conclude that the origin $u=0$ of (322) is locally asymptotically stable. Hence, $(0, \mathbf{v})=(0, \mathbf{0})$ is locally asymptotically stable.

As we mentioned before, the relevant invariant set concerning the future asymptotic dynamics is the set $X_{2}=X_{3}=$ $X_{4}=0$ where the dynamics is given by

$$
\begin{aligned}
& \frac{d X}{d \tau}=\sqrt{6} Y^{2} s \sqrt{1-X^{2}-Y^{2}}-3 X\left(2 X^{2}-1\right)\left(X^{2}+Y^{2}-1\right) \\
& \frac{d Y}{d \tau}=-X Y\left(\sqrt{6} s \sqrt{1-X^{2}-Y^{2}}+6 X\left(X^{2}+Y^{2}-1\right)\right)
\end{aligned}
$$

According the our center manifold calculation the center manifold of the origin can be approximated by the graph 
$x_{2}=x_{3}=x_{4}=0, x=\sqrt{\frac{2}{3}} s y^{2}+\frac{4}{3} \sqrt{\frac{2}{3}} s^{3} y^{4}$, or, using the compact variables, by

$$
\begin{aligned}
X_{2} & =X_{3}=X_{4}=0, \\
X & \left(-X^{2}-Y^{2}+1\right)^{9 / 2}=\sqrt{\frac{2}{3}} s\left(X^{2}-1\right)^{4} Y^{2} \\
& -\frac{4}{3} \sqrt{\frac{2}{3}} s\left(s^{2}-3\right)\left(X^{2}-1\right)^{3} Y^{4} \\
& +\frac{2}{3} \sqrt{\frac{2}{3}} s\left(8 s^{4}-7 s^{2}+9\right)\left(X^{2}-1\right)^{2} Y^{6} \\
& -\frac{4}{27} \sqrt{\frac{2}{3}} s\left(224 s^{6}-126 s^{4}+36 s^{2}-27\right)\left(X^{2}-1\right) Y^{8} \\
& +\frac{1}{81} \sqrt{\frac{2}{3}} s\left(2\left(10880 s^{6}-5088 s^{4}+666 s^{2}-81\right) s^{2}+81\right) Y^{10} .
\end{aligned}
$$

This curve is denoted by a solid line in the Fig. 14, where it is presented a compact phase portrait of the system (323), (324) for different choices of the parameter $s$. For $s=0$ the line $P_{26}\left(y_{c}\right)=\left(0,0,0,0, y_{c}\right)$ is the attractor. For $s \neq 0$ the attractor is the origin.

We see that for small enough $X$-value the curve is a good approximation of the center manifold of the ori-
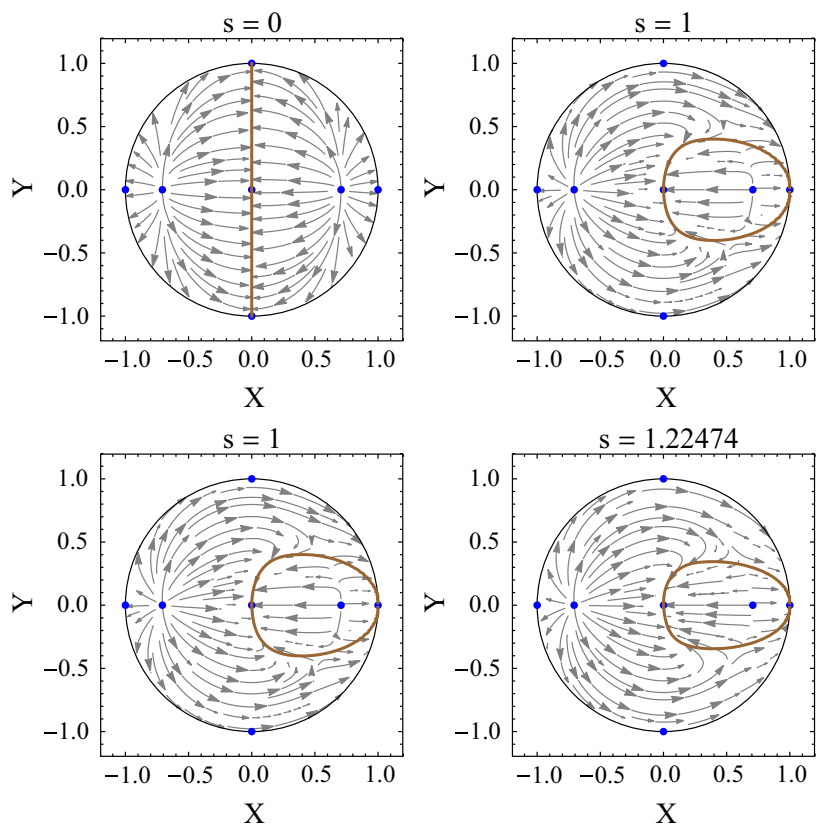

Fig. 14 Compact phase portrait of the system (323), (324), for different choices of the parameter $s$

Finally, it can be implemented the cubic transformation

$$
\left(\begin{array}{c}
x_{2} \\
x_{3} \\
x_{4} \\
x \\
y
\end{array}\right) \rightarrow\left(\begin{array}{c}
x_{2}+x_{2}\left[\left(x+x_{2}+x_{3}\right)^{2}-x_{4}^{2}\right] \\
x_{3}+x_{3}\left[\left(x+x_{2}+x_{3}\right)^{2}-x_{4}^{2}\right] \\
x_{4}+\frac{1}{24}\left[-12 x_{4}^{3}+9\left(x+x_{2}+x_{3}\right)^{2} x_{4}+96 s^{2} y^{2} x_{4}+8 \sqrt{6} s\left(x_{3}-2 x\right) y^{2}\right] \\
x+x\left[\left(x+x_{2}+x_{3}\right)^{2}-x_{4}^{2}\right] \\
y+\frac{y\left\{945 x^{2}+42\left(45 x_{2}+45 x_{3}-16 \sqrt{6} s x_{4}\right) x+5\left\{189 x_{2}^{2}+14\left(27 x_{3}-8 \sqrt{6} s x_{4}\right) x_{2}+3\left\{63 x_{3}^{2}-40 \sqrt{6} s x_{4} x_{3}+28\left[\left(2 s^{2}-3\right) x_{4}^{2}-12 s^{2} y^{2}\right]\right\}\right\}\right\}}{2520}
\end{array}\right),
$$

gin. The advantages of using Center Manifold theory are unveiled immediately when the procedure is compared with the Normal Forms Calculations [129] presented by Leon and Saridakis in [41]. First it is taken a linear transformation $\left(x_{2}, x_{3}, x_{4}, x, y\right) \rightarrow\left(x, x_{3}, x_{2}, x_{4}, y\right)$ to transform the linear part of the system to its real Jordan form: diag $(-6,-4,-3,-2,0)$. Taking the quadratic transformation

$$
\left(\begin{array}{c}
x_{2} \\
x_{3} \\
x_{4} \\
x \\
y
\end{array}\right) \rightarrow\left(\begin{array}{c}
x_{2}-x_{2}\left(x+x_{2}+x_{3}\right) \\
x_{3}-x_{3}\left(x+x_{2}+x_{3}\right) \\
x_{4}+\sqrt{\frac{2}{3}} s y^{2}-\frac{1}{2}\left(x+x_{2}+x_{3}\right) x_{4} \\
x-x\left(x+x_{2}+x_{3}\right) \\
y-\frac{1}{6}\left(3 x+3 x_{2}+3 x_{3}-2 \sqrt{6} s x_{4}\right) y
\end{array}\right)
$$

are eliminated the non resonant terms of second order. resulting in the simplified system

$$
\begin{aligned}
& \frac{d x_{2}}{d N}=-6 x_{2}+\mathscr{O}(4) \\
& \frac{d x_{3}}{d N}=-4 x_{3}+\mathscr{O}(4) \\
& \frac{d x_{4}}{d N}=x_{4}\left(-3+4 s^{2} y^{2}\right)+\mathscr{O}(4), \\
& \frac{d x}{d N}=-2 x+\mathscr{O}(4) \\
& \frac{d y}{d N}=-2 s^{2} y^{3}+\mathscr{O}(4)
\end{aligned}
$$

where $\mathscr{O}(4)$ denotes terms of fourth order in the vector norm. Therefore, the local center manifold of the origin $W_{\text {loc }}^{c}(\mathbf{0})$, is tangent to the $y$-axis at the origin and it can be represented locally up to fourth order as the graph 


$$
\begin{aligned}
& W_{\mathrm{loc}}^{c}=\left\{\left(x_{2}, x_{3}, x_{4}, x, y\right) \in \mathbb{R}^{5}: x_{2}=x_{20} e^{-\frac{3}{2 s^{2} y^{2}}}, x_{3}=x_{30} e^{-\frac{1}{s^{2} y^{2}}},\right. \\
& \left.x_{4}=x_{40} y^{-2} e^{-\frac{1}{s^{2} y^{2}}}, x=x_{0} e^{-\frac{1}{2 s^{2} y^{2}}},|y|<\varepsilon\right\}
\end{aligned}
$$

where $\varepsilon \ll 1$. An it follows under the initial condition $y(0)=$ $y_{0}$, that $y(N)=y_{0}\left(1+4 s^{2} y_{0}^{2} N\right)^{-1 / 2}$, therefore $P_{26}$ is the late-time attractor.

The center manifold, on the other hand, is more economical in the use of computing resources and one obtains a system, say (322), with a reduced dimensionality (1D), when compared with normal forms (5D). Anyway, one can complement both results to find information about the dynamics of a model at hand as for example in [130].

\subsection{Powerlaw potential}

The system (291), (292), (293), (294), (295), (296) becomes:

$$
\begin{aligned}
& \frac{d x_{2}}{d N}=2 x_{2}\left(3 x^{2}+x_{2}+2 x_{3}+3 x_{4}-1\right), \\
& \frac{d x_{3}}{d N}=2 x_{3}\left(3 x^{2}+x_{2}+2 x_{3}+3 x_{4}-2\right), \\
& \frac{d x_{4}}{d N}=2 x_{4}\left(3 x^{2}+x_{2}+2 x_{3}+3 x_{4}-3\right), \\
& \frac{d x}{d N}=3 x^{3}+\left(x_{2}+2 x_{3}+3 x_{4}-3\right) x+\sqrt{6} s y^{2}, \\
& \frac{d y}{d N}=\left(3 x^{2}-\sqrt{6} s x+x_{2}+2 x_{3}+3 x_{4}\right) y, \\
& \frac{d s}{d N}=\frac{\sqrt{6}}{n} x s^{2},
\end{aligned}
$$

defining a dynamical system in $\mathbb{R}^{6}$. The equilibrium points at the finite region and their stability conditions are summarized as follows.

$-\left(x_{2}, x_{3}, x_{4}, x, y, s\right)=\left(0,0,0,0, y_{c}, 0\right)$ with eigenvalues $-6,-4,-3,-2,0,0$. Nonhyperbolic with $2 \mathrm{D}$ center manifold and 4D stable manifold.

- $\left(x_{2}, x_{3}, x_{4}, x, y, s\right)=\left(0,0,1-x_{c}^{2}, x_{c}, 0,0\right)$ with eigenvalues $6,4,3,2,0,0$. Nonhyperbolic with $2 \mathrm{D}$ center manifold and 4D unstable manifold.

$-\left(x_{2}, x_{3}, x_{4}, x, y, s\right)=\left(0,0,0,0,0, s_{c}\right)$. The eigenvalues are $0,0,-6,-4,-3,-2$. Nonhyperbolic with $2 \mathrm{D}$ center manifold and 4D stable manifold.

$-\left(x_{2}, x_{3}, x_{4}, x, y, s\right)=\left(0,0,1,0,0, s_{c}\right)$. The eigenvalues are $0,0,2,3,4,6$. Nonhyperbolic with $2 \mathrm{D}$ center manifold and 4D unstable manifold.

$-\left(x_{2}, x_{3}, x_{4}, x, y, s\right)=\left(0,1,0,0,0, s_{c}\right)$. The eigenvalues are $0,-2,-1,2,2,4$. Nonhyperbolic. It behaves as a saddle.

- $\left(x_{2}, x_{3}, x_{4}, x, y, s\right)=\left(1,0,0,0,0, s_{c}\right)$. The eigenvalues are $0,-4,-2,-2,1,2$. Nonhyperbolic. It behaves as a saddle.
- $\left(x_{2}, x_{3}, x_{4}, x, y, s\right)=(0,0,0,-1,0,0)$. The eigenvalues are $6,4,3,2,0,0$. Nonhyperbolic with $2 \mathrm{D}$ center manifold and 4D unstable manifold.

$-\left(x_{2}, x_{3}, x_{4}, x, y, s\right)=(0,0,0,0,0,0)$. The eigenvalues are $-6,-4,-3,-2,0,0$. Nonhyperbolic with $2 \mathrm{D}$ center manifold and 4D stable manifold.

- $\left(x_{2}, x_{3}, x_{4}, x, y, s\right)=(0,1,0,0,0,0)$. The eigenvalues are $4,-2,2,2,-1,0$. Nonhyperbolic. It behaves as a saddle.

- $\left(x_{2}, x_{3}, x_{4}, x, y, s\right)=(1,0,0,0,0,0)$. The eigenvalues are $-4,-2,-2,2,1,0$. Nonhyperbolic. It behaves as a saddle.

- $\left(x_{2}, x_{3}, x_{4}, x, y, s\right)=(0,0,0,1,0,0)$. The eigenvalues are $6,4,3,2,0,0$. Nonhyperbolic with $2 \mathrm{D}$ center manifold and 4D unstable manifold.

Concerning the dynamics on the infinity region of the phase space, we have found that the relevant asymptotic dynamics occurs on the invariant set $X_{2}=X_{3}=X_{4}=0$, where the evolution equations reduced, after taking a time rescaling, to

$$
\frac{d X}{d \tau}=\sqrt{6} Y^{2} \tan \left(\frac{\pi S}{2}\right)+3 X\left(2 X^{2}-1\right) \sqrt{1-X^{2}-Y^{2}}
$$

$\frac{d Y}{d \tau}=-X Y\left(\sqrt{6} \tan \left(\frac{\pi S}{2}\right)-6 X \sqrt{1-X^{2}-Y^{2}}\right)$,

$\frac{d S}{d \tau}=-\frac{\sqrt{6} X(\cos (\pi S)-1)}{\pi n}$.

Summarizing, we have that the equilibrium points/lines with the higher dimension of the stable manifold are $\left(0,0,0,0, y_{c}, 0\right),\left(0,0,0,0,0, s_{c}\right)$ and $(0,0,0,0,0,0)$. We distinguish the three cases by assuming $y_{c} \neq 0, s_{c} \neq 0$. For all these solutions the center manifold is $2 \mathrm{D}$. Therefore, one can be interested on the stability analysis of the corresponding center manifolds, since they might contain the relevant late-time attractors. On the other hand, we also see that the equilibrium points/lines with the higher dimension of the unstable manifold are $\left(0,0,1-x_{c}^{2}, x_{c}, 0,0\right)$, $\left(0,0,1,0,0, s_{c}\right),(0,0,0,-1,0,0)$, and $(0,0,0,1,0,0)$. We distinguish the four cases. As before, one can be interested on the stability analysis of the corresponding center manifolds, since they might contain the relevant early-time attractors.

To analyze the dynamics on the cylinder at infinity it is more convenient to use the parametrization

$$
X=\sin \left(\frac{\pi \theta}{2}\right), Y=\cos \left(\frac{\pi \theta}{2}\right), \theta \in[-1,1] \text {. }
$$

We consider only the sector $Y \geq 0$ due to the system is invariant under the reflection $Y \rightarrow-Y$. Therefore, we obtain the system 


$$
\begin{aligned}
& \frac{d \theta}{d \tau}=\frac{2 \sqrt{6} \cos \left(\frac{\pi \theta}{2}\right) \tan \left(\frac{\pi S}{2}\right)}{\pi}, \\
& \frac{d S}{d \tau}=-\frac{\sqrt{6} \sin \left(\frac{\pi \theta}{2}\right)(\cos (\pi S)-1)}{\pi n} .
\end{aligned}
$$

In the Fig. 15 it is shown (a) a compact phase portrait of (338), (339), (340) for the choice $n=2$. (b) Dynamics on the cylinder at infinity given by Eqs. (342), (343) for some values of $n$.

\subsection{E-models}

The dynamics on the finite region of the phase space is given by:

$$
\begin{aligned}
& \frac{d x_{2}}{d N}=2 x_{2}\left(3 x^{2}+x_{2}+2 x_{3}+3 x_{4}-1\right), \\
& \frac{d x_{3}}{d N}=2 x_{3}\left(3 x^{2}+x_{2}+2 x_{3}+3 x_{4}-2\right), \\
& \frac{d x_{4}}{d N}=2 x_{4}\left(3 x^{2}+x_{2}+2 x_{3}+3 x_{4}-3\right), \\
& \frac{d x}{d N}=3 x^{3}+\left(x_{2}+2 x_{3}+3 x_{4}-3\right) x+\sqrt{6} s y^{2}, \\
& \frac{d y}{d N}=\left(3 x^{2}-\sqrt{6} s x+x_{2}+2 x_{3}+3 x_{4}\right) y, \\
& \frac{d s}{d N}=\frac{\sqrt{6}}{n} x s(s-\sqrt{6} \mu),
\end{aligned}
$$

defining a dynamical system in $\mathbb{R}^{6}$.

The equilibrium points at the finite region and their stability conditions are summarized as follows.

- $\left(x_{2}, x_{3}, x_{4}, x, y, s\right)=\left(0,0,0,0, y_{c}, 0\right)$, with eigenvalues $-6,-4,-3,-2,0,0$. Nonhyperbolic with $2 \mathrm{D}$ center manifold and 4D stable manifold.

- $\left(x_{2}, x_{3}, x_{4}, x, y, s\right)=\left(0,0,1-x_{c}^{2}, x_{c}, 0, \sqrt{6} \mu\right)$, with eigenvalues $0,2,4,6, \frac{6 x_{c} \mu}{n}, 3-6 x_{c} \mu$. Nonhyperbolic with a 5D unstable manifold for $0<x_{c} \mu<\frac{1}{2}$. Nonhyperbolic and behaves as saddle otherwise.

- $\left(x_{2}, x_{3}, x_{4}, x, y, s\right)=\left(0,0,1-x_{c}^{2}, x_{c}, 0,0\right)$, with eigenvalues $0,2,3,4,6,-\frac{6 x_{c} \mu}{n}$. Nonhyperbolic with a $5 \mathrm{D}$ unstable manifold for $x_{c} \mu<0$. Nonhyperbolic and behaves as saddle otherwise.

- $\left(x_{2}, x_{3}, x_{4}, x, y, s\right)=\left(0,0,0,0,0, s_{c}\right)$, with eigenvalues $0,0,-6,-4,-3,-2$. Nonhyperbolic with $2 \mathrm{D}$ center manifold and 4D stable manifold.

- $\left(x_{2}, x_{3}, x_{4}, x, y, s\right)=\left(0,0,1,0,0, s_{c}\right)$, with eigenvalues $0,0,2,3,4,6$. Nonhyperbolic with $2 \mathrm{D}$ center manifold and 4D unstable manifold.

- $\left(x_{2}, x_{3}, x_{4}, x, y, s\right)=\left(0,1,0,0,0, s_{c}\right)$, with eigenvalues $0,-2,-1,2,2,4$. Nonhyperbolic, behaves as a Saddle.
- $\left(x_{2}, x_{3}, x_{4}, x, y, s\right)=\left(1,0,0,0,0, s_{c}\right)$, with eigenvalues $0,-4,-2,-2,1,2$. Nonhyperbolic, behaves as a Saddle.

$-\left(x_{2}, x_{3}, x_{4}, x, y, s\right)=(0,0,0,-1,0, \sqrt{6} \mu)$, with eigenvalues $0,2,4,6,-\frac{6 \mu}{n}, 6 \mu+3$. Nonhyperbolic with a $5 \mathrm{D}$ unstable manifold for $-\frac{1}{2}<\mu<0$. Nonhyperbolic and behaves as saddle otherwise.

- $\left(x_{2}, x_{3}, x_{4}, x, y, s\right)=(0,0,0,0,0, \sqrt{6} \mu)$, with eigenvalues $-6,-4,-3,-2,0,0$. Nonhyperbolic with $2 \mathrm{D}$ center manifold and 4D stable manifold.

- $\left(x_{2}, x_{3}, x_{4}, x, y, s\right)=(0,1,0,0,0, \sqrt{6} \mu)$, with eigenvalues $4,-2,2,2,-1,0$. Nonhyperbolic, behaves as a Saddle.

- $\left(x_{2}, x_{3}, x_{4}, x, y, s\right)=(1,0,0,0,0, \sqrt{6} \mu)$, with eigenvalues $-4,-2,-2,2,1,0$. Nonhyperbolic, behaves as a Saddle.

- $\left(x_{2}, x_{3}, x_{4}, x, y, s\right)=(0,0,0,1,0, \sqrt{6} \mu)$, with eigenvalues $0,2,4,6, \frac{6 \mu}{n}, 3-6 \mu$. Nonhyperbolic with a 5D unstable manifold for $0<\mu<\frac{1}{2}$. Nonhyperbolic and behaves as saddle otherwise.

- $\left(0,0,1-\frac{1}{4 \mu^{2}}, \frac{1}{2 \mu}, 0, \sqrt{6} \mu\right)$, with eigenvalues 0,0 , $\frac{3}{n}, 2,4,6$. Nonhyperbolic with 2D center manifold and $4 \mathrm{D}$ unstable manifold.

$-\left(x_{2}, x_{3}, x_{4}, x, y, s\right)=\left(1-\frac{1}{12 \mu^{2}}, 0,0, \frac{1}{6 \mu},-\frac{1}{3 \sqrt{2} \mu}\right.$, $\sqrt{6} \mu$ ), with eigenvalues $-4,-2, \frac{1}{n}, 2,-\frac{\sqrt{\mu^{4}-9 \mu^{6}}}{\sqrt{3} \mu^{3}}-1$, $\frac{\sqrt{\mu^{4}-9 \mu^{6}}}{\sqrt{3} \mu^{3}}-1$. Saddle.

- $\left(0,1-\frac{1}{6 \mu^{2}}, 0, \frac{1}{3 \mu},-\frac{1}{3 \sqrt{2} \mu}, \sqrt{6} \mu\right)$, with eigenvalues $-2, \frac{2}{n}, 2,4,-\frac{\sqrt{\frac{8 \mu^{4}}{3}-15 \mu^{6}}}{2 \mu^{3}}-\frac{1}{2}, \frac{\sqrt{\frac{8 \mu^{4}}{3}-15 \mu^{6}}}{2 \mu^{3}}-\frac{1}{2}$. Saddle.

- $\left(x_{2}, x_{3}, x_{4}, x, y, s\right)=\left(1-\frac{1}{12 \mu^{2}}, 0,0, \frac{1}{6 \mu}, \frac{1}{3 \sqrt{2} \mu}, \sqrt{6} \mu\right)$, with eigenvalues $-4,-2, \frac{1}{n}, 2,-\frac{\sqrt{\mu^{4}-9 \mu^{6}}}{\sqrt{3} \mu^{3}}-1$, $\frac{\sqrt{\mu^{4}-9 \mu^{6}}}{\sqrt{3} \mu^{3}}-1$. Saddle.

$-\left(0,1-\frac{1}{6 \mu^{2}}, 0, \frac{1}{3 \mu}, \frac{1}{3 \sqrt{2} \mu}, \sqrt{6} \mu\right)$, with eigenvalues $-2, \frac{2}{n}, 2,4,-\frac{\sqrt{\frac{8 \mu^{4}}{3}-15 \lambda^{6}}}{2 \mu^{3}}-\frac{1}{2}, \frac{\sqrt{\frac{8 \mu^{4}}{3}-15 \mu^{6}}}{2 \mu^{3}}-\frac{1}{2}$. Saddle.

$-\left(x_{2}, x_{3}, x_{4}, x, y, s\right)=\left(0,0,0,2 \mu,-\sqrt{1-4 \mu^{2}}, \sqrt{6} \mu\right)$, with eigenvalues $\frac{12 \mu^{2}}{n}, 24 \mu^{2}, 24 \mu^{2}-6,24 \mu^{2}-4,24 \mu^{2}-$ $2,12 \mu^{2}-3$. Saddle.

$-\left(x_{2}, x_{3}, x_{4}, x, y, s\right)=\left(0,0,0,2 \mu, \sqrt{1-4 \mu^{2}}, \sqrt{6} \mu\right)$, with eigenvalues $\frac{12 \mu^{2}}{n}, 24 \mu^{2}, 24 \mu^{2}-6,24 \mu^{2}-4,24 \mu^{2}-$ $2,12 \mu^{2}-3$. Saddle.

- $\left(x_{2}, x_{3}, x_{4}, x, y, s\right)=(0,0,0,-1,0,0)$, with eigenvalues $0,2,3,4,6, \frac{6 \mu}{n}$. Nonhyperbolic with a 5D unstable manifold for $\mu>0$. Nonhyperbolic and behaves as saddle otherwise. 
Fig. 15 a Compact phase portrait of (338), (339), (340) for the choice $n=2$. b

Dynamics on the cylinder at infinity given by Eqs. (342), (343) for some values of $n$

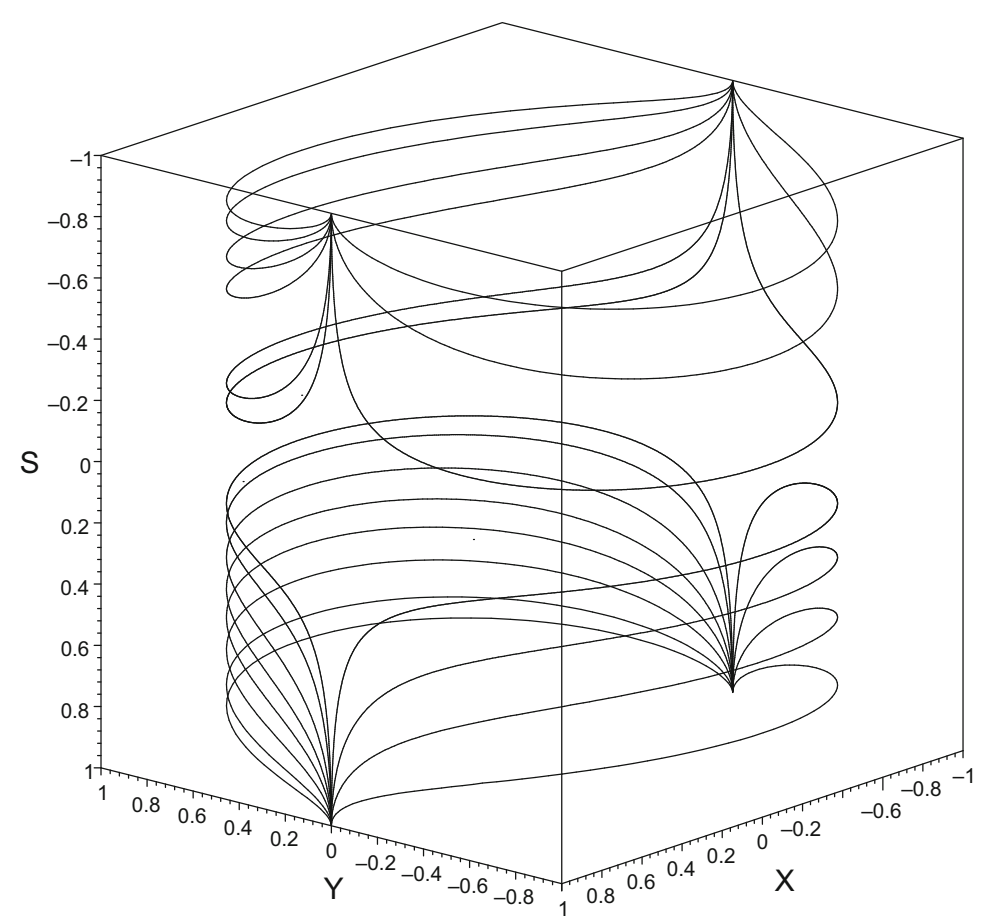

(a)
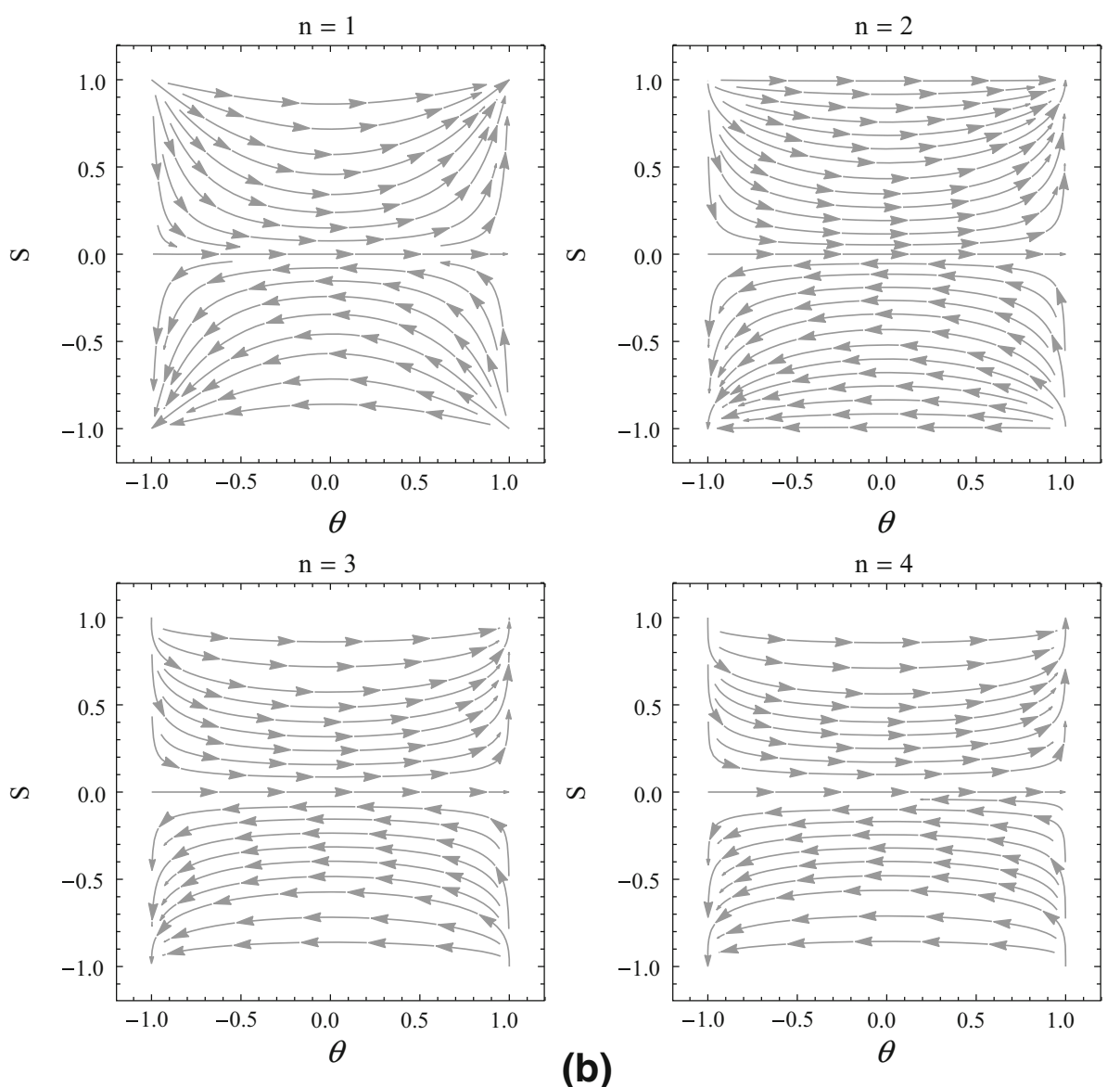
- $\left(x_{2}, x_{3}, x_{4}, x, y, s\right)=(0,0,0,0,0,0)$, with eigenvalues $-6,-4,-3,-2,0,0$. Nonhyperbolic with 2D center manifold and 4D stable manifold.

- $\left(x_{2}, x_{3}, x_{4}, x, y, s\right)=(0,1,0,0,0,0)$, with eigenvalues $4,-2,2,2,-1,0$. Nonhyperbolic and behaves as saddle.

- $\left(x_{2}, x_{3}, x_{4}, x, y, s\right)=(1,0,0,0,0,0)$, with eigenvalues $-4,-2,-2,2,1,0$. Nonhyperbolic and behaves as saddle.

- $\left(x_{2}, x_{3}, x_{4}, x, y, s\right)=(0,0,0,1,0,0)$, with eigenvalues $0,2,3,4,6,-\frac{6 \mu}{n}$. Nonhyperbolic with a $5 \mathrm{D}$ unstable manifold for $\mu<0$. Nonhyperbolic and behaves as saddle otherwise.

The relevant asymptotic dynamics occurs on the invariant set $X_{2}=X_{3}=X_{4}=0$, where the evolution equations reduced, after taking a time rescaling, to

$$
\begin{aligned}
& \frac{d X}{d \tau}=\sqrt{6} Y^{2} \tan \left(\frac{\pi S}{2}\right)+3 X\left(2 X^{2}-1\right) \sqrt{1-X^{2}-Y^{2}} \\
& \frac{d Y}{d \tau}=-X Y\left(\sqrt{6} \tan \left(\frac{\pi S}{2}\right)-6 X \sqrt{1-X^{2}-Y^{2}}\right) \\
& \frac{d S}{d \tau}=\frac{X(-6 \mu \sin (\pi S)-\sqrt{6} \cos (\pi S)+\sqrt{6})}{\pi n}
\end{aligned}
$$

Summarizing, the equilibrium points/lines with the higher dimension of the stable manifold are $\left(0,0,0,0, y_{c}, 0\right)$, $(0,0,0,0,0, \sqrt{6} \mu)$, and $(0,0,0,0,0,0)$. For all these solutions the center manifold is $2 \mathrm{D}$. Therefore, one can be interested on the stability analysis of the corresponding center manifolds, since they might contain the relevant late-time attractors. Whereas, the equilibrium points/lines with the higher dimension of the unstable manifold are $\left(0,0,1-x_{c}^{2}, x_{c}, 0, \sqrt{6} \mu\right),\left(0,0,1-x_{c}^{2}, x_{c}, 0,0\right),(0,0,0$, $-1,0, \sqrt{6} \mu),(0,0,0,1,0, \sqrt{6} \mu),(0,0,0,-1,0,0),(0,0$, $0,1,0,0)$. For all these solutions the center manifold is $1 \mathrm{D}$ and the unstable manifold is 5D. Therefore, one can be interested on the stability analysis of the corresponding center manifolds, since they might contain the relevant early-time attractors.

To analyze the dynamics on the cylinder at infinity it is more convenient to use the parametrization

$X=\sin \left(\frac{\pi \theta}{2}\right), Y=\cos \left(\frac{\pi \theta}{2}\right), \theta \in[-1,1]$.

We consider only the sector $Y \geq 0$ due to the system is invariant under the reflection $Y \rightarrow-Y$. Therefore, we obtain the system

$\frac{d \theta}{d \tau}=\frac{2 \sqrt{6} \cos \left(\frac{\pi \theta}{2}\right) \tan \left(\frac{\pi S}{2}\right)}{\pi}$,

$$
\frac{d S}{d \tau}=-\frac{\sqrt{6} \sin \left(\frac{\pi \theta}{2}\right)(\sqrt{6} \mu \sin (\pi S)+\cos (\pi S)-1)}{\pi n} .
$$

In the Fig. 16 it is shown (a) a compact phase portrait of (350), (351), (352) for the choice $n=2, \mu=1$. (b) Dynamics on the cylinder at infinity given by Eqs. (354), (355) for some values of $n, \mu$.

\section{Conclusions}

Although the HL gravity theory in the original presentation has several drawbacks, still there are several attempts to cure some of these problems. In [32] the authors reconsidered the "detailed balance" as a principle that can be used to restrict the proliferation of couplings in HL gravity, and were discussed some resolutions for all of the usual shortcomings within this framework with the most persistent related to the projectability. The main issues are quite tough to be solved, including how to derive the RG flows, the calculation of higher order quantum corrections, and how to recover Lorentz invariance. The fact some of these issues are very difficult to be answered, however, does not spoil the interest in the theory. In this paper we have proceeded to a very detailed analysis of the stability of several cosmological solutions that are of interest for inflation in HL gravity, using the Center Manifold Theory. We restricted our attention to few aspects of the theory, and we have focused more about the mathematical tools used for the analysis of the solution space of the models. We studied the dynamics of a scalar field in Hořava-Lifshitz cosmology for a wide range of selfinteracting potentials for the scalar field under the detailed balance condition and without imposing it, by means of the powerful method of $f$-devisers. By following this approach one can study the cosmological consequences of the model at hand without the need of an a priori specification of the potential, and in the end one just substitutes the specific potential form in the results, instead of having to repeat the whole dynamical elaboration from the start. As we have seen, the results are richer and more general, revealing the full capabilities of HL cosmology.

Because of the complexity of our analysis we categorized it in four possible cases: (a) $k=0, \Lambda=0$, (b) $k \neq 0, \Lambda=0$, (c) $k=0, \Lambda \neq 0$, and (d) $k \neq 0, \Lambda \neq 0$. For a general potential, the number of equations for the autonomous dimensionless dynamical system under the detailed-balance condition in the three first cases is three, and four for case (d), however, the geometry and the constraint conditions of the phase space change. Due to in general the designed phase spaces are non compact, we have implemented a compactification procedure such that the dynamics in both the finite and infinity region can be visualized with the help of $2 \mathrm{D}$ and 
Fig. 16 a Compact phase portrait of (350), (351), (352) for the choice $n=2, \mu=1$. b Dynamics on the cylinder at infinity given by Eqs. (354), (355) for some values of $n, \mu$

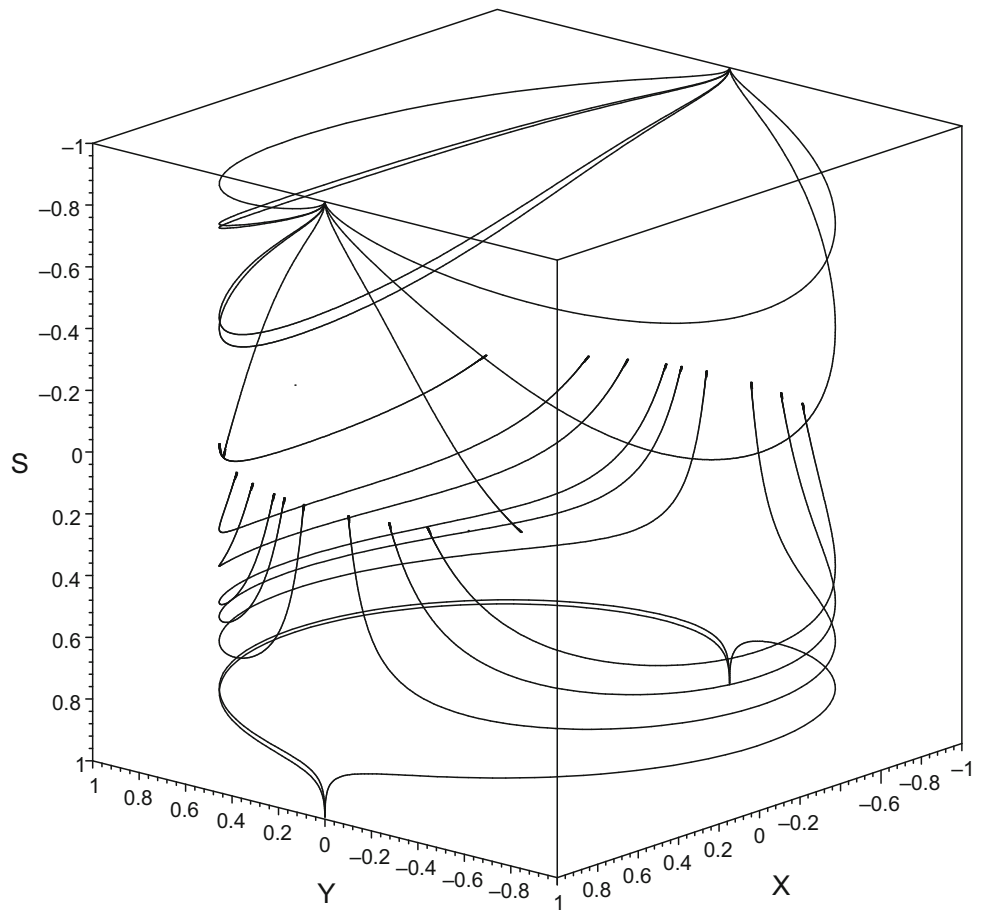

(a)
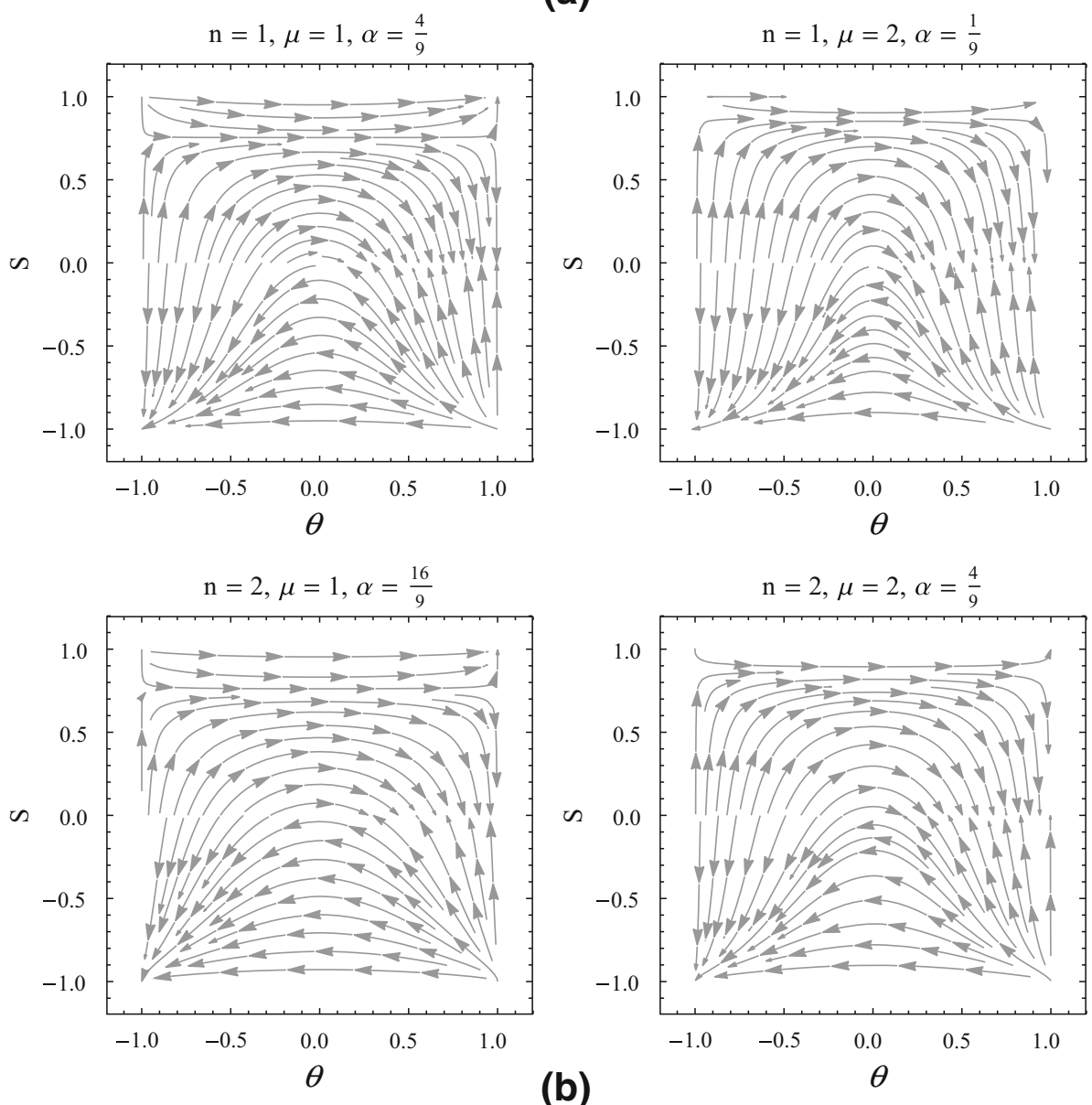
3D phase space by integrating numerically for some specific potentials.

We considered also the case beyond the detailed-balance condition. In that analysis we studied the critical points for a six dimensional dynamical system. For the majority of the critical points the stability depend on the function form of the $f$-devisers. Hence, in order to demonstrate our results we considered some specific forms for the functions $f(s)$, which follows from specific potentials $V(\phi)$ proposed before in the literature.

Specifically, we considered the exponential potential and for potentials beyond the exponential one, e.g., the powerlaw potential $V(\phi)=\frac{1}{2 n}(\mu \phi)^{2 n}, \mu>0, n=1,2, \ldots$ [104] with $f(s)=-\frac{s^{2}}{2 n}$; the potential of the so-called E-model: $V(\phi)=V_{0}\left(1-e^{-\sqrt{\frac{2}{3 \alpha}} \phi}\right)^{2 n}$ with $f(s)=-\frac{s(s-\sqrt{6} \mu)}{2 n}$, $\mu=\frac{2 n}{3 \sqrt{\alpha}}$, discussed in [105] for a conventional scalar field cosmology. Observe that the dynamics of the latter potential is equivalent to that of the exponential potential plus a cosmological constant, $V=V_{0} e^{-\sqrt{6} \mu \phi}+\Lambda$ having $f(s)=-s(s-\sqrt{6} \mu)$, up to a rescaling in the independent variable. Finally, for the exponential potential since we found that in all cases critical points which describe de Sitter universes exist. We performed a thorough analysis on the stability of that solutions with special interests by applying the center manifold theorem. The previous findings by Leon \& Saridakis were recovered and extended and new examples were presented and discussed. The advantages of using Center Manifold theory are unveiled immediately when the procedure is compared with the Normal Forms Calculations presented previously in the literature. The results of [104,105] were recovered as particular cases. We have presented several results concerning the stability of the de Sitter solution in Hořava-Lifshitz cosmology using the Center Manifold theory. We find that in all the cases, with the exception of the model studied in Sect. 7.2.1, the de Sitter solution is unstable: saddle or center-saddle. In Sect. 7.2.1 we proved that the de Sitter solution is locally asymptotically stable. These analyzes are of mathematical relevance for the cosmology.

Acknowledgements This work was funded by Comisión Nacional de Investigación Científica y Tecnológica (CONICYT) through FONDECYT Iniciación 11180126. The author thanks to Departmento de Matemática and to Vicerrectoría de Investigación y Desarrollo Tecnológico at Universidad Católica del Norte for financial support.

Data Availability Statement This manuscript has no associated data or the data will not be deposited. [Authors' comment: This is a theoretical study and no experimental data have been listed.]

Open Access This article is distributed under the terms of the Creative Commons Attribution 4.0 International License (http://creativecomm ons.org/licenses/by/4.0/), which permits unrestricted use, distribution, and reproduction in any medium, provided you give appropriate credit to the original author(s) and the source, provide a link to the Creative
Commons license, and indicate if changes were made. Funded by SCOAP ${ }^{3}$.

\section{Appendix A: Center manifold theory}

In this appendix we summarize the main techniques for the construction of center manifolds for vector fields in $\mathbb{R}^{n}$. We follow the approach of the book [129], chapter 18. The setup is as follows. We consider vector fields in the form

$\mathbf{x}^{\prime}=\mathbf{A} \mathbf{x}+\mathbf{f}(\mathbf{x}, \mathbf{y})$,

$\mathbf{y}^{\prime}=\mathbf{B x}+\mathbf{g}(\mathbf{x}, \mathbf{y}),(\mathbf{x}, \mathbf{y}) \in \mathbb{R}^{c} \times \mathbb{R}^{s}$,

where

$\mathbf{f}(\mathbf{0}, \mathbf{0})=\mathbf{0}, \operatorname{Df}(\mathbf{0}, \mathbf{0})=\mathbf{0}, \mathbf{g}(\mathbf{0}, \mathbf{0})=\mathbf{0}, \operatorname{Dg}(\mathbf{0}, \mathbf{0})=\mathbf{0}$.

A is a $c \times c$ matrix having eigenvalues with zero real parts, $\mathbf{B}$ is an $s \times s$ matrix having eigenvalues with negative real parts, and $\mathbf{f}$ and $\mathbf{g}$ are $C^{r}$ functions $(r \geq 2)$.

Definition 1 (Center manifold) An invariant manifold will be called a center manifold for (A.1) if it can locally be represented as follows

$$
\begin{aligned}
W^{c}(\mathbf{0}) & =\left\{(\mathbf{x}, \mathbf{y}) \in \mathbb{R}^{c} \times \mathbb{R}^{s}: \mathbf{y}=\mathbf{h}(\mathbf{x}),|\mathbf{x}|<\delta\right\} \\
\mathbf{h}(\mathbf{0}) & =\mathbf{0}, \quad D \mathbf{h}(\mathbf{0})=\mathbf{0},
\end{aligned}
$$

for $\delta$ sufficiently small (cf. [129] p. 246, [131], p. 155).

The conditions $\mathbf{h}(\mathbf{0})=\mathbf{0}, D \mathbf{h}(\mathbf{0})=\mathbf{0}$ imply that $W^{c}(\mathbf{0})$ is tangent to $E^{c}$ at $(\mathbf{x}, \mathbf{y})=(\mathbf{0}, \mathbf{0})$, where $E^{c}$ is the generalized eigenspace whose corresponding eigenvalues have zero real parts. The following three theorems (see Theorems 18.1.2, 18.1.3 and 18.1.4 in [129] p. 245-248) are the main results to the treatment of center manifolds. The first two are existence and stability theorems of the center manifold for (A.1) at the origin. The third theorem allows to compute the center manifold to any desired degree accuracy by using Taylor series to solve a quasilinear partial differential equation that $\mathbf{h}(\mathbf{x})$ must satisfy. The proofs of these results are given in [132].

Theorem 1 (Existence) There exists a $C^{r}$ center manifold for (A.1). The dynamics of (A.1) restricted to the center manifold is, for $\mathbf{u}$ sufficiently small, given by the following c-dimensional vector field

$\mathbf{u}^{\prime}=\mathbf{A u}+\mathbf{f}(\mathbf{u}, \mathbf{h}(\mathbf{u})), \mathbf{u} \in \mathbb{R}^{c}$.

The next results implies that the dynamics of (A.4) near $\mathbf{u}=$ 0 determine the dynamics of (A.1) near $(\mathbf{x}, \mathbf{y})=(\mathbf{0 , 0})$ (see also Theorem 3.2.2 in [133]). 
Theorem 2 (Stability) (i) Suppose the zero solution of (A.4) is stable (asymptotically stable) (unstable); then the zero solution of (A.1) is also stable (asymptotically stable) (unstable). Then if $(\mathbf{x}(\tau), \mathbf{y}(\tau))$ is a solution of (A.1) with $(\mathbf{x}(0), \mathbf{y}(0))$ sufficiently small, then there is a solution $\mathbf{u}(\tau)$ of (A.4) such that, as $\tau \rightarrow \infty$

$\mathbf{x}(\tau)=\mathbf{u}(\tau)+\mathscr{O}\left(e^{-r \tau}\right)$,

$\mathbf{x}(\tau)=\mathbf{h}(\mathbf{u}(\tau))+\mathscr{O}\left(e^{-r \tau}\right)$,

where $r>0$ is a constant.

Dynamics Captured by the center manifold. Theorem 2 says that for initial conditions of the full system sufficiently close to the origin, trajectories through them asymptotically approach a trajectory on the center manifold. In particular, singular points sufficiently close to the origin, sufficiently small amplitude periodic orbits, as well as small homoclinic and heteroclinic orbits are contained in the center manifold.

The obvious question now is how to compute the center manifold so that we can use the result of Theorem 2? To answer this question we will derive an equation that $\mathbf{h}(\mathbf{x})$ must satisfy in order to its graph to be a center manifold for (A.1).

Suppose we have a center manifold

$$
\begin{aligned}
W^{c}(\mathbf{0}) & =\left\{(\mathbf{x}, \mathbf{y}) \in \mathbb{R}^{c} \times \mathbb{R}^{s}: \mathbf{y}=\mathbf{h}(\mathbf{x}),|\mathbf{x}|<\delta\right\} ; \\
\mathbf{h}(\mathbf{0}) & =\mathbf{0}, \quad D \mathbf{h}(\mathbf{0})=\mathbf{0},
\end{aligned}
$$

with $\delta$ sufficiently small. Using the invariance of $W^{c}(\mathbf{0})$ under the dynamics of (A.1), we derive a quasilinear partial differential equation that $\mathbf{h}(\mathbf{x})$ must satisfy. This is done as follows:

1. The $(\mathbf{x}, \mathbf{y})$ coordinates of any point on $W^{c}(\mathbf{0})$ must satisfy

$$
\mathbf{y}=\mathbf{h}(\mathbf{x})
$$

2. Differentiating (A.6) with respect to time implies that the $\left(\mathbf{x}^{\prime}, \mathbf{y}^{\prime}\right)$ coordinates of any point on $W^{c}(\mathbf{0})$ must satisfy

$$
\mathbf{y}^{\prime}=D \mathbf{h}(\mathbf{x}) \mathbf{x}^{\prime}
$$

3. Any point in $W^{c}(\mathbf{0})$ obey the dynamics generated by (A.1). Therefore substituting

$$
\mathbf{x}^{\prime}=\mathbf{A x}+\mathbf{f}(\mathbf{x}, \mathbf{h}(\mathbf{x}))
$$$$
\mathbf{y}^{\prime}=\mathbf{B h}(\mathbf{x})+\mathbf{g}(\mathbf{x}, \mathbf{h}(\mathbf{x}))
$$

into (A.7) gives

$$
\begin{aligned}
& \mathscr{N}(\mathbf{h}(\mathbf{x})) \equiv D \mathbf{h}(\mathbf{x})[\mathbf{A x}+\mathbf{f}(\mathbf{x}, \mathbf{h}(\mathbf{x}))]-\mathbf{B h}(\mathbf{x}) \\
& -\mathbf{g}(\mathbf{x}, \mathbf{h}(\mathbf{x}))=0
\end{aligned}
$$

Equation (A.10) is a quasilinear partial differential that $\mathbf{h}(\mathbf{x})$ must satisfy in order for its graph to be an invariant center manifold. To find the center manifold, all we need to do is solve (A.10). Unfortunately, it is probably more difficult to solve (A.10) than our original problem; however the following theorem give us a method for computing an approximated solution of (A.10) to any desired degree of accuracy.

Theorem 3 (Approximation) Let $\Phi: \mathbb{R}^{c} \rightarrow \mathbb{R}^{s}$ be a $C^{1}$ mapping with $\Phi(\mathbf{0})=\mathbf{0}$ and $D \Phi(\mathbf{0})=\mathbf{0}$ such that $\mathscr{N}(\Phi(\mathbf{x}))=\mathscr{O}\left(\|\mathbf{x}\|^{q}\right)$ as $\mathbf{x} \rightarrow \mathbf{0}$ for some $q>1$. Then, $|\mathbf{h}(\mathbf{x})-\Phi(\mathbf{x})|=\mathscr{O}\left(\|\mathbf{x}\|^{q}\right)$ as $\mathbf{x} \rightarrow \mathbf{0}$.

This theorem allows us to compute the center manifold to any desired degree of accuracy by solving (A.10) to the same degree of accuracy, and we can use power series expansions.

\section{References}

1. P. Horava, Phys. Rev. D 79, 084008 (2009)

2. T. Jacobson, D. Mattingly, Phys. Rev. D 64, 024028 (2001)

3. C. Eling, T. Jacobson, D. Mattingly, arXiv:gr-qc/0410001

4. W. Donnelly, T. Jacobson, Phys. Rev. D 82, 064032 (2010)

5. S. Kanno, J. Soda, Phys. Rev. D 74, 063505 (2006)

6. T.G. Zlosnik, P.G. Ferreira, G.D. Starkman, Phys. Rev. D 75, 044017 (2007)

7. I. Carruthers, T. Jacobson, Phys. Rev. D 83, 024034 (2011)

8. T. Jacobson, PoS QG -PH 020 (2007)

9. S.M. Carroll, E.A. Lim, Phys. Rev. D 70, 123525 (2004)

10. D. Garfinkle, T. Jacobson, Phys. Rev. Lett. 107, 191102 (2011)

11. T. Jacobson, Phys. Rev. D 89, 081501 (2014)

12. T.P. Sotiriou, J. Phys. Conf. Ser. 283, 012034 (2011)

13. R.G. Cai, Y. Liu, Y.W. Sun, JHEP 0906, 010 (2009)

14. R.G. Cai, B. Hu, H.B. Zhang, Phys. Rev. D 80, 041501 (2009)

15. C. Charmousis, G. Niz, A. Padilla, P.M. Saffin, JHEP 0908, 070 (2009)

16. C. Bogdanos, E.N. Saridakis, Class. Quant. Gravit. 27, 075005 (2010)

17. M. Henneaux, A. Kleinschmidt, G. Lucena Gómez, Phys. Rev. D 81, 064002 (2010)

18. K. Koyama, F. Arroja, JHEP 1003, 061 (2010)

19. J. Bellorin, B. Droguett, Phys. Rev. D 98(8), 086008 (2018)

20. J. Bellorin, A. Restuccia, Gen. Relat. Gravit. 49(10), 132 (2017)

21. J. Bellorin, A. Restuccia, A. Sotomayor, J. Phys. Conf. Ser. 831(1), 012002 (2017)

22. J. Bellorin, A. Restuccia, Int. J. Mod. Phys. D 27(01), 1750174 (2017)

23. J. Bellorin, A. Restuccia, A. Sotomayor, J. Phys. Conf. Ser. 738(1), 012041 (2016)

24. J. Bellorin, A. Restuccia, Phys. Rev. D 94(6), 064041 (2016)

25. J. Bellorin, A. Restuccia, A. Sotomayor, Int. J. Mod. Phys. D 25(02), 1650016 (2015)

26. J. Bellorin, A. Restuccia, A. Sotomayor, Phys. Rev. D 90(4), 044009 (2014)

27. A. Restuccia, J. Bellorin, A. Sotomayor, J. Phys. Conf. Ser. 490, 012123 (2014)

28. J. Bellorin, A. Restuccia, A. Sotomayor, Phys. Rev. D 87(8), 084020 (2013)

29. J. Bellorin, A. Restuccia, A. Sotomayor, Phys. Rev. D 85, 124060 (2012)

30. J. Bellorin, A. Restuccia, Phys. Rev. D 84, 104037 (2011) 
31. J. Bellorin, A. Restuccia, Int. J. Mod. Phys. D 21, 1250029 (2012)

32. D. Vernieri, T.P. Sotiriou, Phys. Rev. D 85, 064003 (2012)

33. D. Vernieri, Phys. Rev. D 91(12), 124029 (2015)

34. E. Kiritsis, G. Kofinas, Nucl. Phys. B 821, 467 (2009)

35. G. Calcagni, JHEP 0909, 112 (2009)

36. D. Blas, O. Pujolas, S. Sibiryakov, Phys. Rev. Lett. 104, 181302 (2010)

37. D. Blas, O. Pujolas, S. Sibiryakov, JHEP 0910, 029 (2009)

38. T. Christodoulakis, N. Dimakis, J. Geom. Phys. 62, 2401 (2012)

39. N. Frusciante, M. Raveri, D. Vernieri, B. Hu, A. Silvestri, Phys. Dark Univ. 13, 7 (2016)

40. H. Lu, J. Mei, C.N. Pope, Phys. Rev. Lett. 103, 091301 (2009)

41. G. Leon, E.N. Saridakis, JCAP 0911, 006 (2009)

42. S. Mukohyama, K. Nakayama, F. Takahashi, S. Yokoyama, Phys. Lett. B 679, 6 (2009)

43. E.N. Saridakis, Int. J. Mod. Phys. D 20, 1485 (2011)

44. A. Ali, S. Dutta, E.N. Saridakis, A.A. Sen, Gen. Relat. Gravit. 44, 657 (2012)

45. S. Nojiri, S.D. Odintsov, Phys. Rev. D 81, 043001 (2010)

46. S. Mukohyama, JCAP 0906, 001 (2009)

47. B. Chen, S. Pi, J.Z. Tang, JCAP 0908, 007 (2009)

48. Y.S. Piao, Phys. Lett. B 681, 1 (2009)

49. B. Chen, S. Pi, J.Z. Tang, JCAP 0908, 007 (2009)

50. U.H. Danielsson, L. Thorlacius, JHEP 0903, 070 (2009)

51. R.G. Cai, L.M. Cao, N. Ohta, Phys. Rev. D 80, 024003 (2009)

52. A. Ghodsi, E. Hatefi, Phys. Rev. D 81, 044016 (2010)

53. A. Kehagias, K. Sfetsos, Phys. Lett. B 678, 123 (2009)

54. E.N. Saridakis, Gen. Relat. Gravit. 45, 387 (2013)

55. E.N. Saridakis, Eur. Phys. J. C 67, 229 (2010)

56. S. Dutta, E.N. Saridakis, JCAP 1001, 013 (2010)

57. S. Dutta, E.N. Saridakis, JCAP 1005, 013 (2010)

58. S.S. Kim, T. Kim, Y. Kim, Phys. Rev. D 80, 124002 (2009)

59. R.G. Cai, L.M. Cao, N. Ohta, Phys. Lett. B 679, 504 (2009)

60. M. Jamil, E.N. Saridakis, M.R. Setare, JCAP 1011, 032 (2010)

61. M. Jamil, E.N. Saridakis, JCAP 1007, 028 (2010)

62. R. Brandenberger, Phys. Rev. D 80, 043516 (2009)

63. Y.F. Cai, E.N. Saridakis, JCAP 0910, 020 (2009)

64. M. Khodadi, Y. Heydarzade, F. Darabi, E.N. Saridakis, Phys. Rev. D 93(12), 124019 (2016)

65. S.F. Bramberger, A. Coates, J. Magueijo, S. Mukohyama, R. Namba, Y. Watanabe, Phys. Rev. D 97(4), 043512 (2018)

66. J. Bellorin, A. Restuccia, F. Tello-Ortiz, Phys. Rev. D 98(10), 104018 (2018)

67. E.M.C. Abreu, A.C.R. Mendes, G. Oliveira-Neto, J. Ananias Neto, L.G.R. Rodrigues, M. Silva De Oliveira, arXiv:1805.11042 [gr-qc]

68. R. Maier, I.D. Soares, Phys. Rev. D 96(10), 103532 (2017). Addendum: [Phys. Rev. D 97(4), 049902 (2018)]

69. A. Wang, Int. J. Mod. Phys. D 26(07), 1730014 (2017)

70. N.A. Nilsson, E. Czuchry, Phys. Dark Univ. 23, 100253 (2019)

71. A.E. Gumrukcuoglu, M. Saravani, T.P. Sotiriou, Phys. Rev. D 97, 024032 (2018)

72. A. Wang, Y. Wu, JCAP 0907, 012 (2009)

73. M.M. Anber, J.F. Donoghue, Phys. Rev. D 83, 105027 (2011)

74. M. Pospelov, Y. Shang, Phys. Rev. D 85, 105001 (2012)

75. S. Groot Nibbelink, M. Pospelov, Phys. Rev. Lett. 94, 081601 (2005)

76. A. Coates, C. Melby-Thompson, S. Mukohyama, arXiv:1805.10299 [hep-th]

77. G. D’Odorico, F. Saueressig, M. Schutten, Phys. Rev. Lett. 113(17), 171101 (2014)

78. T. Griffin, P. Horava, C.M. Merby-Thompson, Phys. Rev. Lett. 110, 081602 (2013)

79. A.O. Barvinsky, D. Blas, M. Herrero-Valea, S.M. Sibiryakov, C.F. Steinwachs, Phys. Rev. D 93, 064022 (2016)
80. A.O. Barvinsky, D. Blas, M. Herrero-Valea, S.M. Sibiryakov, C.F. Steinwachs, JHEP 1807, 035 (2018)

81. A. Contillo, S. Rechenberger, F. Saueressig, JHEP 12, 017 (2013)

82. S. Mukohyama, Class. Quant. Gravit. 27, 223101 (2010)

83. I. Kimpton, A. Padilla, Matter in Horava-Lifshitz gravity. JHEP 04, 133 (2013)

84. D. Vernieri, S. Carloni, EPL 121(3), 30002 (2018)

85. D. Vernieri, arXiv:1906.07738 [gr-qc]

86. A. Paliathanasis, G. Leon, arXiv:1903.10821 [gr-qc]

87. D. Vernieri, Phys. Rev. D 98(2), 024051 (2018)

88. V.G. LeBlanc, D. Kerr, J. Wainwright, Class. Quant. Gravit. 12, 513-541 (1995)

89. J.M. Heinzle, C. Uggla. Class. Quant. Gravit. 27:513-541, 015009 (2010)

90. J. Wainwright, G.F.R. Ellis (eds). Dynamical Systems in Cosmology. (Cambridge University Press, Cambridge, 1997) p. 343

91. A.A. Coley, arXiv:gr-qc/9910074

92. A.A. Coley, (Kluwer, Dordrecht, 2003), p. 200

93. S. Carloni, E. Elizalde, P.J. Silva, Class. Quant. Gravit. 27, 045004 (2010)

94. M.R. Setare, D. Momeni, Int. J. Theor. Phys. 50, 106 (2011)

95. V. Cardoso, A.S. Miranda, E. Berti, H. Witek, V.T. Zanchin, Phys. Rev. D 79, 064016 (2009)

96. A.G. Lavkin, Sov. J. Nucl. Phys. 52, 759-760 (1990)

97. T.C. Charters, A. Nunes, J.P. Mimoso, Class. Quant. Gravit. 18, 1703-1714 (2001)

98. I.Y. Aref'eva, N.V. Bulatov, S.Y. Vernov, Theor. Math. Phys. 163, 788-803

99. D. Escobar, C.R. Fadragas, G. Leon, Y. Leyva, Astrophys. Space Sci. 349, 575 (2014)

100. C.R. Fadragas, G. Leon, E.N. Saridakis, Class. Quant. Gravit. 31, 075018 (2014)

101. G. Papagiannopoulos, S. Basilakos, J.D. Barrow, A. Paliathanasis, Phys. Rev. D 97(2), 024026 (2018)

102. A. Paliathanasis, JCAP 1708(08), 027 (2017)

103. L. Karpathopoulos, S. Basilakos, G. Leon, A. Paliathanasis, M. Tsamparlis, Gen. Relat. Gravit. 50, 79 (2018)

104. A. Alho, J. Hell, C. Uggla, Class. Quant. Gravit. 32(14), 145005 (2015)

105. A. Alho, C. Uggla, Phys. Rev. D 95(8), 083517 (2017)

106. J. Yearsley, J.D. Barrow, Class. Quant. Gravit. 13, 2693 (1996)

107. R. Cardenas, T. Gonzalez, Y. Leiva, O. Martin, I. Quiros, Phys. Rev. D 67, 083501 (2003)

108. S.A. Pavluchenko, Phys. Rev. D 67, 103518 (2003)

109. V. Sahni, A.A. Starobinsky, Int. J. Mod. Phys. D 9, 373 (2000)

110. B. Ratra, P.J.E. Peebles, Phys. Rev. D 37, 3406 (1988)

111. C. Wetterich, Nucl. Phys. B 302, 668 (1988)

112. E.J. Copeland, S. Mizuno, M. Shaeri, Phys. Rev. D 79, 103515 (2009)

113. Y. Leyva, D. Gonzalez, T. Gonzalez, T. Matos, I. Quiros, Phys. Rev. D 80, 044026 (2009)

114. S. del Campo, C.R. Fadragas, R. Herrera, C. Leiva, G. Leon, J. Saavedra, Phys. Rev. D 88, 023532 (2013)

115. V. Sahni, L.-M. Wang, Phys. Rev. D 62, 103517 (2000)

116. J.E. Lidsey, T. Matos, L.A. Urena-Lopez, Phys. Rev. D 66, 023514 (2002)

117. T. Matos, L.A. Urena-Lopez, Class. Quant. Gravit. 17, L75 (2000)

118. T. Matos, J.-R. Luevano, I. Quiros, L.A. Urena-Lopez, J.A. Vazquez, Phys. Rev. D 80, 123521 (2009)

119. L.A. Urena-Lopez, T. Matos, Phys. Rev. D 62, 081302 (2000)

120. T. Barreiro, E.J. Copeland, N.J. Nunes, Phys. Rev. D 61, 127301 (2000)

121. T. Gonzalez, R. Cardenas, I. Quiros, Y. Leyva, Astrophys. Space Sci. 310, 13 (2007)

122. T. Gonzalez, G. Leon, I. Quiros, Class. Quant. Gravit. 23, 3165 (2006) 
123. S. Mukohyama, Phys. Rev. D 80, 064005 (2009)

124. M. Li, Y. Pang, JHEP 0908, 015 (2009)

125. T.P. Sotiriou, M. Visser, S. Weinfurtner, Phys. Rev. Lett. 102, 251601 (2009)

126. T.P. Sotiriou, M. Visser, S. Weinfurtner, JHEP 0910, 033 (2009)

127. C. Appignani, R. Casadio, S. Shankaranarayanan, JCAP 1004, 006 (2010)

128. E. Kiritsis, Phys. Rev. D 81, 044009 (2010)

129. S. Wiggins, Introduction to Applied Nonlinear Dynamical Systems and Chaos (Springer, New York, 2003)
130. G. Leon, A. Paliathanasis, J.L. Morales-Martínez, Eur. Phys. J. C 78(9), 753 (2018)

131. L. Perko, Differential Equations and Dynamical Systems (Springer, Berlin, 1991)

132. J. Carr, Applications of Center Manifold theory (Springer, New York, 1981)

133. J. Guckenheimer, P. Holmes, Nonlinear Oscillations, Dynamical Systems and Bifurcations of Vector Fields (Springer, New York, 1983) 